\title{
Solid state NMR method development and studies of biological and biomimetic nanocomposites \\ by \\ Yan-Yan Hu
}

\begin{abstract}
A dissertation submitted to the graduate faculty
in partial fulfillment of the requirements for the degree of

DOCTOR OF PHILOSOPHY
\end{abstract}

\author{
Major: Analytical Chemistry \\ Program of Study Committee: \\ Klaus Schmidt-Rohr, Major Professor \\ Mei Hong \\ Surya K. Mallapragada \\ Robert S. Houk \\ Yan Zhao
}

Iowa State University

Ames, Iowa

2011

Copyright (C) Yan-Yan Hu, 2011. All rights reserved 


\section{Table of Contents}

Acknowledgements $\quad$ V

Abstract vii

Chapter 1 Introduction 1

Overview 1

The structure of biological and biomimetic nanocomposites $\quad 1$

Solid state NMR method development and improvement $\quad 8$

$\begin{array}{ll}\text { Thesis organization } & 9\end{array}$

$\begin{array}{ll}\text { Copyright permissions } & 10\end{array}$

$\begin{array}{ll}\text { Appendix } & 10\end{array}$

$\begin{array}{ll}\text { References } & 27\end{array}$

Chapter 2 Strongly bound citrate stabilizes the apatite nanocrystals in bone 36

Abstract 36

Introduction 36

Results and discussion $\quad 37$

$\begin{array}{ll}\text { Conclusions and outlook } & 41\end{array}$

Methods $\quad 42$

Acknowledgements 44

References $\quad 44$

$\begin{array}{ll}\text { Figures } & 48\end{array}$

Supporting information $\quad 53$

Chapter 3 Biomimetic self-assembling nanocomposites of block copolymer and hydroxyapatite with nanocrystal size controlled by citrate $\quad 60$

$\begin{array}{ll}\text { Abstract } & 60\end{array}$

$\begin{array}{ll}\text { Introduction } & 60\end{array}$

Materials and methods $\quad 63$

Results and discussion $\quad 66$ 
$\begin{array}{ll}\text { Conclusions } & 75\end{array}$

$\begin{array}{ll}\text { Acknowledgements } & 76\end{array}$

$\begin{array}{ll}\text { References } & 76\end{array}$

$\begin{array}{ll}\text { Tables and figures } & 79\end{array}$

$\begin{array}{ll}\text { Supporting information } & 90\end{array}$

Chapter 4 Self-assembled calcium phosphate nanocomposites using block copolypeptide templates 92

$\begin{array}{ll}\text { Abstract } & 92\end{array}$

Introduction $\quad 93$

Results and discussion $\quad 95$

$\begin{array}{ll}\text { Materials and methods } & 104\end{array}$

$\begin{array}{ll}\text { Conclusions } & 108\end{array}$

$\begin{array}{ll}\text { Acknowledgements } & 109\end{array}$

$\begin{array}{ll}\text { References } & 109\end{array}$

$\begin{array}{ll}\text { Tables and figures } & 114\end{array}$

$\begin{array}{ll}\text { Supporting information } & 125\end{array}$

Chapter 5 Broadband "infinite-speed" magic-angle spinning NMR

Spectroscopy 126

$\begin{array}{ll}\text { Abstract } & 126\end{array}$

Main text 126

$\begin{array}{ll}\text { Acknowledgements } & 130\end{array}$

$\begin{array}{ll}\text { Figures } & 130\end{array}$

References 132

Chapter 6 Technical aspects of fast magic-angle turning NMR for dilute spin-1/2 nuclei with broad spectra 134

$\begin{array}{ll}\text { Abstract } & 134\end{array}$

Introduction 134 
Materials and methods $\quad 135$

Theoretical background and experimental setup 136

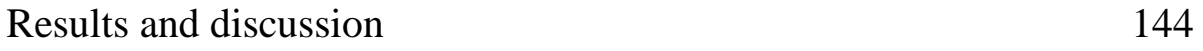

$\begin{array}{ll}\text { Conclusions } & 146\end{array}$

$\begin{array}{ll}\text { Acknowledgements } & 147\end{array}$

$\begin{array}{ll}\text { References } & 147\end{array}$

$\begin{array}{ll}\text { Figures } & 148\end{array}$

Supporting information 156

Chapter 7 Effects of L-spin longitudinal quadrupolar relaxation in $\mathrm{S}\{\mathrm{L}\}$

heteronuclear recoupling and S-spin magic-angle spinning NMR 158

$\begin{array}{ll}\text { Abstract } & 158\end{array}$

$\begin{array}{ll}\text { Introduction } & 159\end{array}$

$\begin{array}{ll}\text { Theoretical background and simulations } & 160\end{array}$

Experimental results and discussion 172

$\begin{array}{ll}\text { Conclusions } & 176\end{array}$

$\begin{array}{ll}\text { Materials and methods } & 176\end{array}$

$\begin{array}{ll}\text { Acknowledgements } & 177\end{array}$

$\begin{array}{ll}\text { Appendices } & 177\end{array}$

References $\quad 182$

$\begin{array}{ll}\text { Tables and figures } & 185\end{array}$

$\begin{array}{lll}\text { Chapter } 8 \quad \text { General conclusions } & 204\end{array}$

$\begin{array}{ll}\text { General discussion } & 204\end{array}$

Recommendations for future research 206 


\section{Acknowledgements}

First and foremost, I would like to express my deep and sincere gratitude to my Ph.D. adviser, Professor Klaus Schmidt-Rohr. It has been truly a privilege and honor to be his graduate student. I appreciate all his contributions of time, effort, ideas, support, motivation, and encouragement to make my Ph.D. experience joyful and productive. He has consciously and unconsciously taught me the attitude, logic, and methodology to conduct good science; the kindness to be always polite, extremely helpful and care for other people's needs; and the clear vision to see what is really important in life. He is there with me to anticipate my professional future and help me to prepare for it with full support and encouragement every

step forward. I am also thankful for the excellent example he has provided me as a dedicated, successful, and well-respected scientist.

I am also indebted to Professor Mei Hong, Professor Surya Mallapragada, and Professor Mufit Akinc for their stimulating discussions and guidance towards my thesis research. I would like to thank Professor Robert S. Houk for those memorable analytical classes filled with his unique humor and his constructive comments and suggestions on my work. Many thanks to Professor Yan Zhao for his kind help to attend my final defense.

I am very grateful to Professor Patricia Thiel for being a great mentor to me: showing me the wonderful side of professorship, being always encouraging and supportive, and teaching me how to network, how to get an easy start of my career, and how to collaborate with people. She has been there with me to make the most important decisions of my life. Most of all, she has been an incredible role model for me as a great scientist, wonderful wife and mother, and a collegial colleague.

The current and past members of Schmidt-Rohr and Hong group have contributed immensely to my professional and personal development. Dr. Aditya Rawal had patiently trained me with the implementation of basic solid state NMR experiments when I first started, Dr. Xiaowen Fang, Dr. Rajee Mani, Dr. Ming Tang, Dr. Timothy Doherty, Dr. Wenbin Luo, Dr. Sarah Cady, and Dr. Shenhui Li had lent me a hand with technical difficulties I encountered in my research. Special thanks to Mr. Yongchao Su, Miss Yuan Zhang, and Dr. Xueqian Kong for their countless help over years. I certainly enjoyed the 
inspiring and encouraging conversations with Dr. Evgenii M. Levin and the pleasant company of Mr. Fanghao Hu, Mrs. Marilu Dick-Perez, and Mr. Robert Johnson.

The warm support and love from all my friends helped me survive long cruel winters in Ames and have a wonderful time along the way. My first roommate Xia Wang and my friend Wenjun Xie initially taught me how to survive in a foreign country and helped me furnish my first apartment. Dancing surely brought me tons of fun along the side of my friends, Becky, Yingjun, and Ben. Training for Marathon with Suzanne Sander every week became my most relaxing time and most rewarding activity provided with Suzanne's invaluable friendship. I surely will miss those delicious dinners prepared by Bosa and Adu accompanied with the laughter brought by Albert and Andrey. I will never forget those delightful moments with my good friends Feng Liu, Yuan Zhang, Lin Liu, and the hospitality of Yan Sun and Wei Qiang during my visit to Michigan and of Beibei to Shanghai.

Special thanks to Sean Nedd for accompanying me through all those good and difficult times, for helping me grow into a strong, independent, and optimistic person, for his consistent support and rescue from all the troubled situations I got myself into, and for his tolerance and understanding.

I am forever grateful to my grandparents and my parents whose foresight and values paved the way for a privileged education and who gently offered counsel and unconditional support and love at each turn of my life. My life could never been this joyful without my loving and caring brother.

This thesis is dedicated to my father and mother. The determination, optimistic spirit, and sense of joy with which they respond to life's challenges have imprinted on me and led me to overcome every hurdle on my journey. Life has blessed me with the opportunity to meet Uncle Shiao-wei, Auntie Fun-Dee and their family. This thesis is a small tribute to an exceptional couple who unconditionally helped me, supported me, and loved me over years. 


\begin{abstract}
This thesis describes application and development of advanced solid-state nuclear magnetic resonance techniques for complex materials, in particular organic-inorganic nanocomposites and thermoelectric tellurides. The apatite-collagen interface, essential for understanding the biomineralization process in bone and engineering the interface for controlled bio-mimetic synthesis and optimized mechanical properties, is buried within the nanocomposite of bone. We used multinuclear solid-state NMR to study the composition and structure of the interface. Citrate has been identified as the main organic molecule strongly bound to the apatite surface with a density of $1 /(2 \mathrm{~nm})^{2}$, covering 1/6 of the total surface area in bovine bone. Citrate provides more carboxylate groups, one of the key functional groups found to affect apatite nucleation and growth, than all the non-collagenous proteins all together in bone; thus we propose that citrate stabilizes apatite crystals at a very small thickness of $\sim 3 \mathrm{~nm}$ (4 unit cells) to increase bone fracture tolerance. The hypothesis has been confirmed in vitro by adding citrate in the bio-mimetic synthesis of polymerhydroxyapatite nanocomposites. The results have shown that the size of hydroxyapatite nanocrystals decreases as increasing citrate concentration. With citrate concentrations comparable to that in body fluids, similar-sized nanocrystals as in bone have been produced. Besides the dimensions of the apatite crystals, the composition of bone also affects its biofunctional and macroscopic mechanical properties; therefore, our team also extended its effort to enhance the inorganic portion in our bio-mimetic synthesis from originally $15 \mathrm{wt} \%$ to current $50 \mathrm{wt} \%$ compared to $65 \mathrm{wt} \%$ in bovine bone, by using Lysine-Leucine hydroxyapatite nucleating diblock co-polypeptide, which forms a gel at very low concentration.

In this thesis, various advanced solid state NMR techniques have been employed to characterize nanocomposites. Meanwhile, we have developed new methods to achieve broadband high resolution NMR and improve the accuracy of inter-nuclear distance measurements involving quadrupolar spins. Broadband high resolution NMR of spin-1/2 nuclei has been accomplished by the adaptation of the magic angle turning (MAT) method to fast magic angle spinning, termed fast MAT, by solving technical problems such as off-
\end{abstract}


resonance effects. Fast MAT separates chemical shift anisotropy and isotropic chemical shifts over a spectral range of $\sim 1.8 \gamma \mathrm{B}_{1}$ without significant distortions. Fast MAT ${ }^{125} \mathrm{Te}$ NMR has been applied to study technologically important telluride materials with spectra spreading up to $190 \mathrm{kHz}$. The signal-to-noise ratio of the spectra is significantly improved by using echo-matched Gaussian filtering in offline data processing. The accuracy of the measured distances between spin-1/2 and quadrupolar nuclei with methods such as SPIDER and REAPDOR has been improved by compensating for the fast longitudinal quadrupolar relaxation on the sub-millisecond with a modified $\mathrm{S}_{0}$ pulse sequence. Also, the $\mathrm{T}_{1 \mathrm{Q}}$ effect on the spin coherence and its spinning speed dependency has been explored and documented with analytical and numerical simulations as well as experimental measurements. 


\section{Chapter 1}

\section{Introduction}

\subsection{Overview}

Solid state nuclear magnetic resonance (NMR) is an advanced technique that allows researchers to see the otherwise invisible world with traditional characterization methods. I first realized and appreciated the power of solid state NMR by applying available NMR methods and data analysis to chemically and materially interesting systems - biological and biomimetic nanocomposites, aiming to push forward our understanding of the compositional and structural design of natural materials such as bone and apply this information to guide biomimetic synthesis for prosthetic applications. Later on, with a little ambition and tremendous help and guidance from my adviser, I have been able to make tiny steps in developing and improving solid state NMR methods: one of the methods is to facilitate the accurate measurement of distances between nuclei, especially when quadrupolar nuclei are involved, by accounting for the fast $\mathrm{T}_{1}$ relaxation due to large quadrupolar couplings; the other one is to promote broadband high resolution NMR by fast magic-angle turning (MAT) experiments.

\subsection{The structure of biological and biomimetic nanocomposites Nanocomposites and their promises}

The research field of nanocomposites encompasses the study of multiphase materials of which at least the length of one dimension is < $100 \mathrm{~nm}$. The promising aspects of nanocomposites reside in their unique functionalities that cannot be achieved in traditional materials. The tremendous challenges to realize the promises of nanocomposites include the precise control over the size distribution and dispersion of the nanosize constituents; understanding the role of interface structure and composition on the bulk chemical and mechanical properties; and the ability of engineering the interface for optimum performances. To overcome these challenges, we resort to our mentor Mother Nature and her exquisite creations of natural composites for answers and clues. One such creation is bone - a hierarchical nanocomposite of collagen and apatite. 


\section{The composition and structure of bone}

The composition, structure, and mechanical properties of bone have been studied

extensively 12345 for the purpose of understanding, of mimicking to produce similarly multi-function materials, and of creating desirable structures and properties beyond nature.

Bone, as the main load-bearing and protective organ in the mammalian body, is constituted of three major components: mineral (carbonated apatite, 45 vol \%), organics (mainly type I collagen with non-collagenous proteins, 45 vol \%), and water (10 vol \%). The mechanical properties, namely modulus of elasticity, yield stress and strain, and fracture mechanism, of bone are largely dependent on the relative amount, the structures, and the spatial arrangements of these three constituents ${ }^{6}$. The naturally optimized synergistic combination of these three components renders bone light-weight yet strong and tough.

\section{Composition and structure of apatite in bone}

The stiffness of bone is mainly provided by the inorganic carbonated apatite. The study of apatite in bone was initiated in 1926 by deJong with X-ray diffraction ${ }^{7}$. Many decades of research has resulted in abundance of information about the structure and composition of apatite in various bones. However, some results are contentious due to the complications derived from the structural and compositional dependencies on crystal ages, locations, treatments, and characterization methods, as well as misinterpretations of experimental data ${ }^{1}$. Generally agreed conclusions will be presented and still elusive problems will be discussed in the following.

The chemical composition of biological apatite is significantly different from ideal hydroxyapatite $(\mathrm{HAp}), \mathrm{Ca}_{10}(\mathrm{PO} 4)_{6}(\mathrm{OH})_{2}$, in that apatite contains a varying amount of $\mathrm{HPO}_{4}{ }^{2-}$ and carbonate ions and only a small percentage of the total stoichiometric number of hydroxyl ions found in ideal HAp ${ }^{89}$. Other ion substitutions were also identified, such as $\mathrm{Mg}$ or $\mathrm{Na}$ for $\mathrm{Ca}$ ions and $\mathrm{F}$ for $\mathrm{OH}$ ions. Cationic and anionic vacancies occur within the

crystal lattice and a general formula was proposed as $\mathrm{Ca}_{10-\mathrm{x}}\left(\mathrm{PO}_{4}\right)_{6-\mathrm{x}}\left(\mathrm{HPO}_{4} \text { or } \mathrm{CO}_{3}\right)_{\mathrm{x}}(\mathrm{OH})_{2-\mathrm{x}} 10$ and confirmed by Rietveld analysis of X-ray diffraction patterns ${ }^{11}$.

Those inorganic ions are not only present within the apatite lattice but also in the hydrated layer on the surface of apatite, a large portion of which are $\mathrm{CO}_{3}{ }^{2-}$ and $\mathrm{HPO}_{4}{ }^{2-}$. The 
hydrated apatite surface layer is referred to as a non-apatitic environment ${ }^{12}{ }^{13}$, but little is known about its composition and structure. Debatable arguments have been made to identify the surface layer as dicalcium phosphate dihydrate (DCPD) by solid state ${ }^{31} \mathrm{P}$ NMR, and octacalcium phostate (OCP) by FTIR and amorphous calcium phosphate (ACP) in dry samples ${ }^{14}$. Nevertheless, these results are either not reproducible or not supported by other characterization methods.

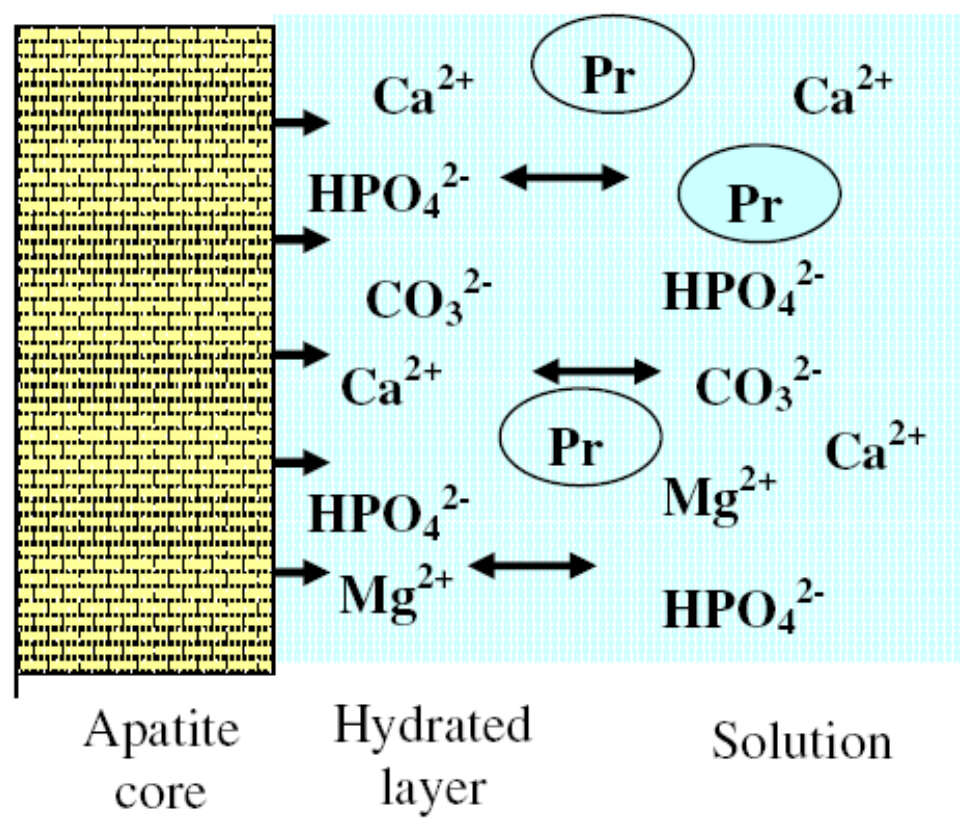

Figure 1.1. Schematic of apatite nanocrystals in bone mineral. The core of apatite crystals is a non-stoichiometric carbonated apatite lattice. The surface is amorphous and hydrated containing loosely bound ions. The ions in the hydrated layer can exchange with those in the surrounding body fluids including charged proteins (Pr), which affects the surface activity of apatite. Figure adapted from Rey et al. ${ }^{1}$

Much effort has been dedicated to discovering the possible precursor phase prior to the formation of poorly crystallized apatite crystals ${ }^{15,16}$. Identifications of OCP or "OCPlike" and ACP were made but were disputed on two bases: the initially formed nanocrystals are very unstable and can be readily transformed to $\mathrm{OCP}$ or ACP upon interaction with water; and the inability of separating the non-apatitic environment and apatite precursors. Recently published work using synchrotron X-ray diffraction and small-angle neutron scattering technique to track the in vivo mineralization process of fin bony rays of zebrafish 
has claimed that OCP nano-spheres (actually identified as ACP) are formed initially, and then they transform to plate-like mature apatite crystals ${ }^{17}$.

Structural studies of bone apatite have been carried out using wide- and small-angle X-ray diffraction (XRD) ${ }^{18 ~ 19-23}$, high resolution transmission electron microscopy (TEM) ${ }^{24}$, atomic force microscopy (AFM) ${ }^{25}$, and solid state NMR ${ }^{26} 27$. The carbonated apatite mineral has a hexagonal symmetry, so normally it is expected that all six faces are developed equally to form needle- or rod-like structures. However, the mature apatite crystals are found indeed as very thin plate-like elongated nanocrystals, with a thickness of $\sim 3 \mathrm{~nm}$ (Fig 1.2); this thickness, comparable to the length of 4 unit cells, is thought to resist bone fracture by preventing crack propagation ${ }^{28}$. This preferentially inhibited growth of the apatite crystal surfaces cannot be merely from spatial constraint imposed by biology. The size of apatite crystals in bone varies with species, location and age within a range in the lengths and thicknesses, thus the growth of apatite has to be somehow regulated. This regulation is very distinct from solution-mediated Ca phosphate precipitation and geologic apatite formation. The question now becomes what initiates the nucleation of apatite crystals, regulates their growth, and stabilizes apatite over other calcium phosphates. The answer to this question will help us to meet the challenges of controlled synthesis of nanocomposites and surface engineering for optimal performance.

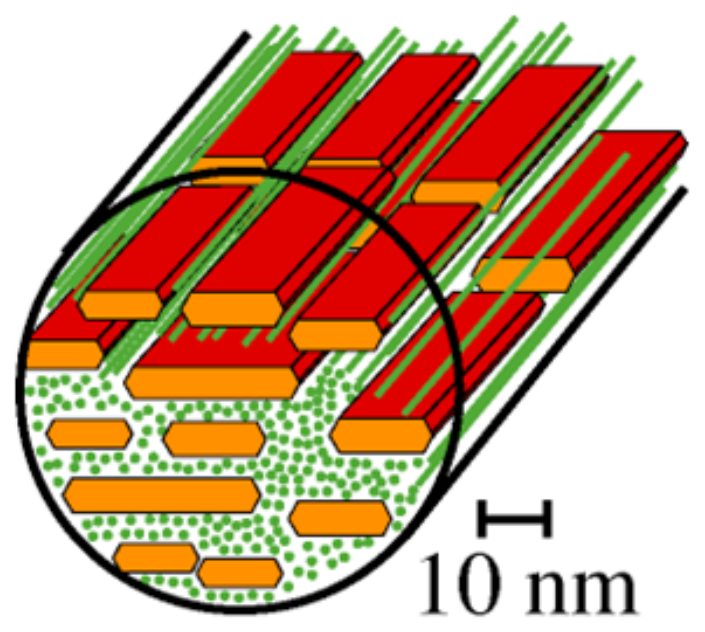

Figure 1.2. Diagram of the bone nanocomposites containing plate-like apatite crystals of $\sim 3$ $\mathrm{nm}$ thick (orange) and collagen fibers (green). Adapted from Rawal ${ }^{27}$ 
Also, certain diseases are associated with the abnormal size or crystallinity of apatite crystals in bone, for instance, reduced crystal size in Paget's disease ${ }^{29}$ and diabetes ${ }^{30}$, but increased in osteopetrotic patients ${ }^{31}$. The answer to what controls bone biomineralization will help to develop therapies for the cure of relevant diseases and fabricate composites similarly structured as bone for advanced tissue engineering. Bioactive organic molecules involved in this process are expected to have the following characteristics: initiate and control the formation of apatite crystals, exist in a sufficient quantity to be effective, and be in close contact with the inorganic apatite phase.

\section{Bio-organic components in bone}

The organic components in bone play important roles in the biochemical, structural, and mechanical properties of bone. Type I collagen accounts for $>90 \%$ of the total organics in bone. The rest are non-collagenous proteins, such as osteocalcin, osteonectin, osteopontin, sialoproteins, proteoglycans, and other phosphoproteins; they only make small contributions to the volume of bone but are important in biomineralization.

Type I collagen is composed of three triple helical peptide chains of approximately 1000 amino acids, each with the repeating unit X-Y-Gly, where $\mathrm{X}$ is usually proline and Y is hydroxyproline ${ }^{32}$. Collagen molecules self-assemble into bundles of collagen fibrils. The fibrils are then grouped to form collagen fibers, which act as the major structural component of bone matrix. In vitro research using collagen separated from bone and purified to remove other attached organics shows no evidence of collagen initiating or regulating the growth of hydroxyapatite crystals ${ }^{34}$, however, it serves as a scaffold to retain noncollagenaceous proteins either promoting or inhibiting apatite growth as discussed in the following.

Osteocalcin is the most abundant non-collagenous protein in bone, accounts for $\sim 15$ $\%$ of the total non-collagenous proteins ${ }^{33}$. Osteocalcin is considered bone-specific protein, since it is only found in bone and dentin. Osteocalcin has a high affinity to Ca-rich apatite and its Gla-domain may bind to the (100) face of apatite crystal, and has therefore been proposed to control the length of apatite crystals ${ }^{34}$.

Matrix Gla-protein (MGP) is another major Gla-containing protein besides osteocalcin, and MGP is phosphorylated, thus it is also an apatite-binding protein. MGP has 
very low solubility in physiologic solutions and tends to self-associate by hydrophobic interactions. MGP is found in vivo to be an inhibitor of mineralization ${ }^{35}$.

Other proteins such as osteopontin ${ }^{36}$, bone-sialoprotein ${ }^{37}$, and osteonectin ${ }^{38}$ all have a high affinity for $\mathrm{Ca}^{2+}$ due to their functional groups such as phosphate or carboxylate; in vitro, they were found to promote hydroxyapatite formation at low concentrations but inhibit its growth at high concentrations. Nevertheless, these proteins are present at less than $3 \mathrm{wt} \%$ of total organics and the number of carboxylate or phosphate functional groups is limited. Moreover, these proteins are only distributed within the "hole" or "pore" zones of the collagen matrix rather than between the collagen and apatite (Fig 1.3) ${ }^{39}$; this distribution limits their interactions with apatite surface.

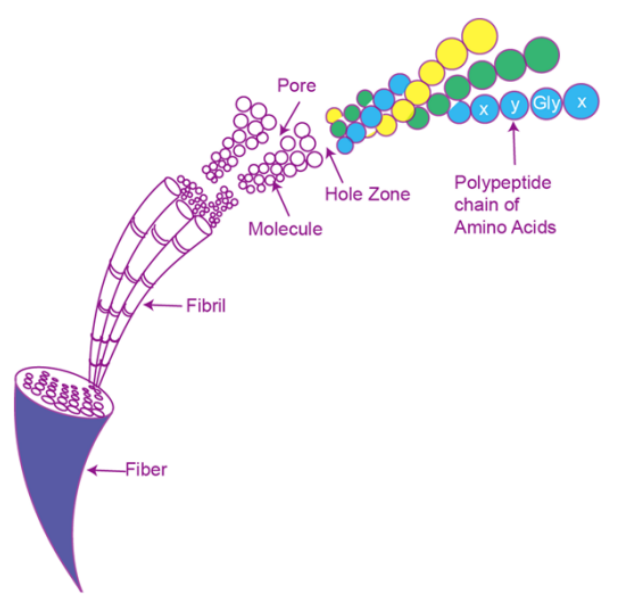

Figure 1.3. The structure of collagen fiber and fibrils with putative locations of pores and "hole" zones shown. Figure adapted from Morgan et al. 39

\section{The interfacial structure in bone and "active" bio-molecules}

The organic-inorganic interface connects the structurally and chemically dissimilar phases and the interfacial modifications prove to largely affect the bulk properties of nanocomposites. Besides, the "active" biomolecules that control the biomineralization of bone tissue are believed to exist at the interface.

Despite the importance of the interface, very little is known largely because the interface is buried within the nanocomposites and very difficult to access with traditional diffraction or microscopic techniques. The chemical extraction of possible "active" molecules is destructive, since it requires total dissolution of the mineral phase. This process 
makes it very difficult to trace the location and orientation of these molecules and their conformation can also be potentially altered.

Advanced solid state NMR is a nondestructive technique that can be used to preferentially select the interfacial carbon moieties from bulk collagen carbons without chemical or physical separation and purification, to determine the structure and conformation of the "active" biomolecules, and to study their distribution density and dynamic properties.

The knowledge of the interfacial composition and structure obtained by solid state NMR complemented by other techniques has been applied to bio-inspired nanocomposite syntheses for three purposes: i) to validate the role in vitro of the identified "active" molecule in bone mineralization; ii) to tailor the interface structure in order to achieve accurate size control for nanocomposite production; and iii) to create bone-like nanostructure with better biocompatibility for tissue engineering and prosthetic applications.

\section{Solid state NMR of bio-mimetic nanocomposites}

Based on the knowledge gleaned from nature, bone-like structure has been created in vitro. The sol-gel method ${ }^{40}$ is chosen to achieve optimal molecular contact between the organic and inorganic phases and closely mimic the process of bone mineralization. Due to their availability and tunability, synthetic polymers are preferred to cells, proteins, and natural polymers to take on the role of collagen in bone matrix ${ }^{41}$. The progress of biomimetic synthesis of polymer-calcium phosphate nanocomposites is marked by three milestones. First, the apatite has been stabilized over other calcium phosphates with $\mathrm{pH}$ and temperature close to physiological conditions, and by using apatite-nucleating organics 4243 44 . Second, the formation of nanocomposites has been achieved with a composition similar to bone ${ }^{45}$. Third, the size of apatite nanocrystals within nanocomposites has been finely tuned to match that in bone. During this process, solid state NMR has played a key role in identifying the phosphate phases, confirming nanocomposite formation, determining the size of the hydroxyapatite crystals and thus guiding the synthesis to reach a higher level. 


\subsection{Solid state NMR method development and improvement}

\section{Fast magic angle turning}

In this thesis, all the experiments were performed under magic angle spinning (MAS) to remove chemical shift anisotropy (CSA) and achieve high resolution NMR. However, if the CSA parameter $\delta_{\mathrm{CSA}}>\omega_{\mathrm{r}}$ (spinning speed), pronounced spinning sidebands are observed. Strong radio frequency pulses $\left(\gamma \mathrm{B}_{1} \sim \delta_{\mathrm{CSA}}\right)$ could be employed to suppress these or separate CSA and isotropic chemical shifts in different dimensions.

Among techniques separating anisotropic and isotropic chemical shifts, Gan's magic angle turning (MAT) ${ }^{46,47}$ experiment was identified here as the best broadband technique due to the use of only short pulses and particularly suitable for high-Z spin-1/2 nuclei such as ${ }^{125} \mathrm{Te},{ }^{195} \mathrm{Pt}$, and ${ }^{207} \mathrm{~Pb}$, which have CSAs of hundreds of $\mathrm{kHz}$ when in non-cubic environments. However, with a peak width on the order of hundreds of $\mathrm{kHz}$, the $\mathrm{T}_{2}{ }^{*}$ is on the order of sub milliseconds, thus fast MAS is required to maximize the sensitivity. The adaptation of MAT to fast MAS is demonstrated on various technologically important tellurides in Chapter 5 and the technical aspects are detailed in Chapter 6.

\section{“REDOR” for quadrupolar nuclear spin and $T_{1 Q}$ relaxation compensation.}

REDOR (Rotational-echo double resonance) ${ }^{54}$ was used as the major solid state NMR technique to select the signals of biomolecules at the apatite-collagen interface and for distance measurements. Instead of REDOR, the recoupling of dipolar coupling interactions involving quadrupolar nuclei under MAS is achieved by applying REAPDOR ${ }^{48}$, TEDOR ${ }^{49}$, SPIDER ${ }^{50}$, or RAPDOR ${ }^{48}$. More often than not, qudrupolar nuclei have short quadrupolar $\mathrm{T}_{1}$ relaxation times $\left(\mathrm{T}_{1 \mathrm{Q}}\right)$ of sub-milliseconds due to large quadrupolar couplings of several MHz. Since the recoupling time in REDOR-like experiments can reach a few milliseconds, then the double quantum coherence term $S_{\mathrm{y}} \mathrm{I}_{\mathrm{z}} e^{-t / T_{1}}$ is significantly relaxed in the first half of the recoupling period and cannot be reconverted back to the observable $\left\langle\mathrm{S}_{\mathrm{x}}\right\rangle$ in the second half of the recoupling period; this causes the reduction or complete loss of the reference $\mathrm{S}_{0}$ intensity. Thus the measured $\mathrm{S} / \mathrm{S}_{0}\left(\mathrm{D}, \mathrm{Nt}_{\mathrm{r}}\right)$ yields underestimated dipolar coupling constant and overestimated inter-nuclear distance. This deviation can be corrected by incorporating 
the $T_{1 Q}$ relaxation into simulations for curve fitting or minimizing $T_{1 Q}$ relaxation during $S_{0}$ by moidifying the pulse sequence as discussed in Chapter 7.

\subsection{Thesis organization}

This thesis describes solid state NMR studies of natural and bio-mimetic nanocomposites as well as improvement and development of NMR methods. Chapter 1 comprises a brief statement of the objectives of this thesis study, a general introduction on the composition and structure of the natural nanocomposite, bone, and difficulties in characterizing the "buried" interface, a description of the bio-mimetic synthesis based on the study of bone and the role of solid state NMR in this process, a discussion of the main solid state NMR methods employed, and relevant method development and improvement.

Chapter 2 presents the solid state NMR study of the collagen-apatite interface in bone: we have identified the main organic component as immobile citrate strongly bound to the apatite surface, analyzed the distance between citrate carbons and calcium in apatite, and determined the density of citrate on the surface of apatite. We propose that citrate inhibits the thickening of apatite crystals and stabilizes them at a thickness of $\sim 3 \mathrm{~nm}$. Based on the study of citrate in bone, in Chapter 3, citrate has been incorporated into the bio-mimetic synthesis of polymer-hydroxyapatite nanocomposites in order to regulate the size of hydroxyapatite crystals. The results have shown that the size of hydroxyapatite crystals decreases with increasing citrate concentration, which in vitro confirms the hypothesis proposed in Chapter 2. Also, at citrate concentrations comparable to those in body fluids, hydroxapatite nanocrystals of similar size as apatite in bone have been produced. Chapter 4 describes the bio-mimetic study to achieve a similar organic-inorganic composition as in bone. Lysineleucine diblock copolypeptide, which can form a gel at very low concentration, was used to template the formation of hydroxyapatite and the highest inorganic content of $55 \mathrm{wt} \%$ has been obtained, compared with $65 \mathrm{wt} \%$ apatite in bone.

Chapter 5 reports the adaptation of magic-angle turning (MAT) to fast MAT, termed fast MAT, and demonstrates fast MAT on technologically important tellurides. The technical aspects, such as simulation and conpensation of off-resonance effects, echo-matched Gaussian filtering, and sharing between channels after shearing, are detailed in Chapter 6. 
Chapter 7 gives a quantitative analysis on the effect of short $\mathrm{T}_{1 \mathrm{Q}}$ of quadrupolar nuclei on REAPDOR and SPIDER measurements and presents a new pulse sequence to minimize the $T_{1 Q}$ relaxation effect on the reference signal $S_{0}$.

\subsection{Copy permissions}

Chapters 2, 4, 5, 7 are reprints of published papers. Copyright permissions have been obtained from the following publishing groups:

Chapter 2: National Academy of Sciences

Chapter 4: The Royal Society of Chemistry

Chapter 5: American Chemical Society

Chapter 7: Elsevier, Inc.

\section{Appendix: Solid state NMR methods for nanocomposite characterization}

Magic Angle Spinning (MAS). Without tumbling motions as in liquid, solid state NMR spectra are broadened due to various interactions, including chemical shift anisotropy, dipolar coupling, and quadrupolar coupling, which disguise the characteristic isotropic chemical shifts. In order to achieve high resolution NMR, magic-angle spinning (MAS) ${ }^{51}$ is often employed to remove chemical shift anisotropy and partially dipolar coupling. All the experiments in this thesis were performed under MAS.

The chemical shift in the magnetic field $\mathrm{B}_{0}$ can be expressed as

$6_{c s}=\begin{gathered}6^{\text {sotjorgic }} \\ \sigma_{i s o} \gamma B_{0}\end{gathered}+\frac{1}{2} \delta_{c s}\left[3 \cos ^{2} \theta-1-\eta_{c s} \sin ^{2} \theta \cos (2 \phi)\right]$,

$\sigma_{i s o}:$ isotropic chemical shift tensor

$\gamma$ : gyromagnetic ratio

$\delta_{\mathrm{cs}}$ : anisotropy parameter

$\eta_{\mathrm{cs}}$ : asymmetry parameter

$\theta$ and $\phi$ : polar coordinates of the magnetic field tensor $\mathrm{B}_{0}$ in the principle axis system (PAS)

which yields powder spectra as shown below. 
MAS

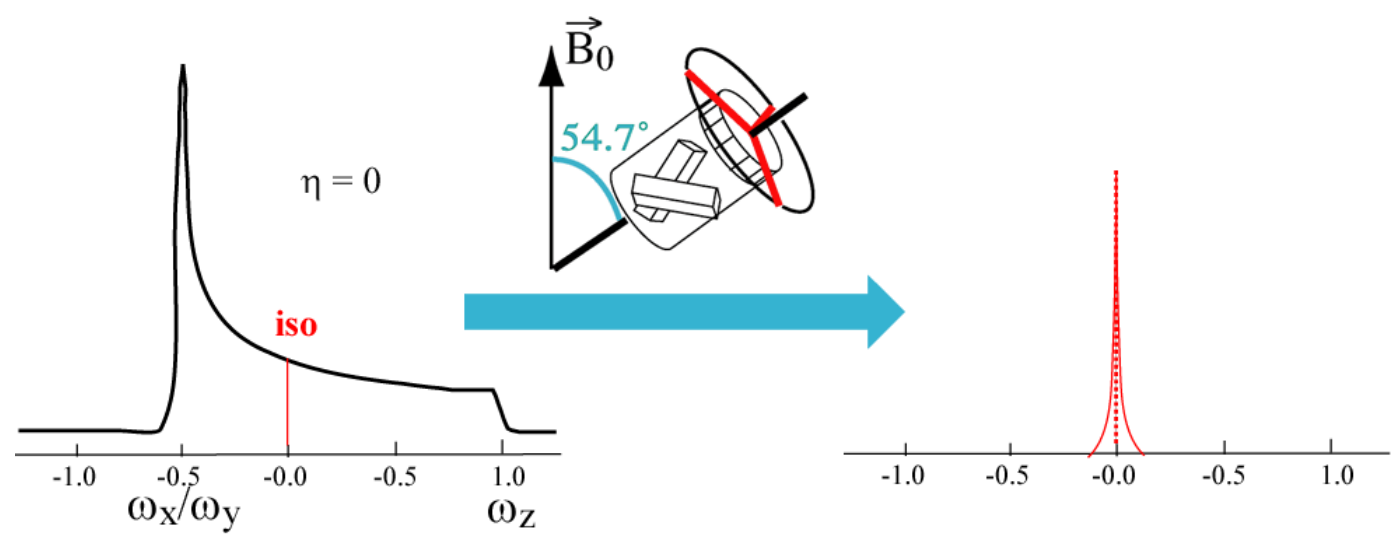

Figure 1.A1. Schematics of a powder pattern, magic angle spinning (MAS), and isotropic chemical shift. The powder pattern shown is with isotropic chemical shift positioned at 0 ppm and the asymmetry parameter of $\eta=0 . \omega_{\mathrm{x}}, \omega_{\mathrm{y}}$, and $\omega_{\mathrm{z}}$ are in units of $\delta$ (the anisotropy parameter). With sufficiently fast MAS, the CSA is removed and the isotropic chemical shift is revealed.

Anisotropic broadening can be eliminated by either reorientations of molecular segments via molecular motions or macroscopic rotation like MAS. MAS is carried out around an axis at the "magic-angle" $\theta=54.74^{\circ}\left(3 \cos ^{2}\left(54.74^{\circ}\right)-1=0\right)$ relative to the magnetic field $\mathrm{B}_{0}$. When the rotor performs fast uniaxial rotation $\left(\bar{\eta}_{m o l}=0\right)$ in MAS, the averaged chemical shift frequency is,

$$
\begin{aligned}
& 6444444 \overline{\bar{g}}_{\text {mol }} 4444448 \\
& \omega=\frac{1}{2}\left(3 \cos ^{2} \beta_{P}-1-\eta \sin ^{2} \beta_{P} \cos 2 \alpha_{P}\right) \delta \frac{1}{2}\left[3 \cos ^{2} \theta_{r}-1\right] \text {. }
\end{aligned}
$$

$\alpha_{P}$ and $\beta_{P}:$ polar angles of the rotation axis in PAS.

$\theta_{\mathrm{r}}$ : the angle between the rotation axis and the $\mathrm{B}_{0}$ field.

If the MAS frequency $\omega_{\mathrm{r}}>>\delta$, any anisotropic interactions will be spun out (e.g. regular MAS for alkyl carbons); if $\omega_{\mathrm{r}} \sim \delta$, incomplete suppression of chemical shift anisotropy will result in spinning sidebands; total sideband suppression (TOSS) ${ }^{52}$ or phase-adjusted sideband suppression (PASS) ${ }^{53}$ are effective for moderate spectral range (e.g. regular MAS 
for aromatic or carboxylate carbons); if $\omega_{\mathrm{r}}<<\delta$, strong radio frequency pulses $\left(\gamma \mathrm{B}_{1} \sim \delta\right)$ can be employed for the separation of CSA and isotropic chemical shifts.

Rotational-echo double resonance (REDOR) and multi-spin simulation. The dipolar coupling in solid state NMR is strongly distance dependent. For a pair of nuclei with spins I and $\mathrm{S}$, the dipolar coupling frequency is

$$
\omega_{D}=\frac{\gamma_{I} \gamma_{S} \eta \mu_{0}}{4 \pi r^{3}} \frac{1}{2}\left(3 \cos ^{2} \theta-1\right)
$$

$\gamma_{\mathrm{I}}, \gamma_{\mathrm{S}}$ : gyromagnetic ratio of spin I and $\mathrm{S}$

$\eta$ : Planck constant

$\mu_{0}:$ permeability of free space

$\theta$ : angle between the dipolar vector and $\mathrm{B}_{0}$ field

which allows for direct determination of internuclear distance. However, in high resolution solid state NMR with MAS, the heteronuclear dipolar interaction is averaged to zero at the end of each rotor period. In order to recouple dipolar couplings, Schaefer and co-workers have introduced Rotational-Echo Double Resonance (REDOR) ${ }^{54}$ and Transferred-Echo Double Resonance (TEDOR) ${ }^{49}$ techniques which apply radio frequency pulses at specific points of the rotor periods to avoid the complete averaging of dipolar coupling by MAS and allow dipolar dephasing to accumulate. With appropriate data analysis, the magnitude of dipolar coupling can be extracted and inter-atomic distances can be determined for systems with isolated spin-pairs, which is the case in selectively- isotopically labeled biomolecules. Very often, a spin interacts with multiple heteronuclear spins, thus multispin analysis is necessary to account for the measured REDOR decay.

In the REDOR pulse sequence, a $\pi$-pulse is placed at every half and complete rotor period to achieve the maximum dephasing. Although in principle, the radio frequency pulses can be applied on either channel, for spin-1/2 nuclei, preferably the number of pulses on the observe channel is minimized to reduce the effects of pulse-error accumulation and $\mathrm{B}_{1}$ inhomogeneity on the intensity of observed signals. 


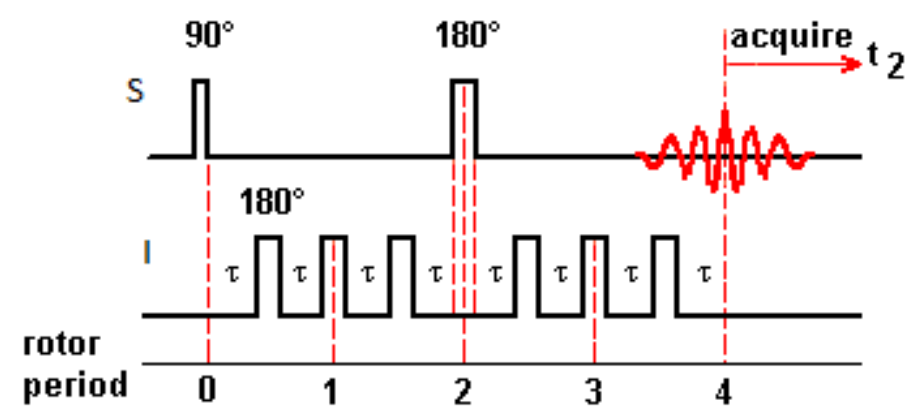

Figure 1.A2. REDOR pulse sequence with a train of $\pi$-pulses applied to the unobservable channel I and a $\pi$-pulse in the middle of the recoupling period on the observable channel $\mathrm{S}$ for chemical shift refocusing. The reference signal $\mathrm{S}_{0}$ is acquired with an additional $\pi$-pulse in the middle of the recoupling period on the I-channel.

The dephased REDOR signal $\mathrm{S}$ is measured with the pulse sequence shown in Figure 1.A2. The reference signal $S_{0}$ is also acquired by adding an extra $\pi$-pulse in the middle of the recoupling period on the I-channel to cancel the dipolar coupling effect on the magnitude of the observed signal and measure the decays caused by $\mathrm{T}_{2}$ relaxation, non-ideal pulses, and $\mathrm{B}_{1}$ inhomegeneity and fluctuation.

The dipolar coupling frequency $\omega_{\mathrm{D}}$ becomes time-dependent under MAS and in the interaction frame of the REDOR pulse sequence, for the first half of the rotor period,

$$
\omega_{D}(\gamma, \beta, t)=\frac{\gamma_{I} \gamma_{S} \eta \mu_{0}}{4 \pi r^{3}} \frac{1}{2}\left\{\left(\sin ^{2} \beta \cos \left(2 \gamma+2 \omega_{r} t\right)-\sqrt{2} \sin 2 \beta \cos \left(\gamma+\omega_{r} t\right)\right\} .\right.
$$

With $\pi$-pulse in the middle of the rotor period,

$$
\int_{t_{r} / 2}^{t_{r}} \omega(\gamma, \beta, t) d t=\int_{0}^{t_{r} / 2} \omega(\gamma, \beta, t) d t
$$

After one rotor period with REDOR,

$$
\overline{\omega_{D}(\gamma, \beta, t)}=2 \int_{0}^{t_{r} / 2} \omega(\gamma, \beta, t) d t / t_{r}=\frac{\gamma_{I} \gamma_{S} \eta \mu_{0}}{4 \pi r^{3}} \frac{1}{2} \frac{\sqrt{2}}{\pi} \sin 2 \beta \sin \alpha .
$$

The evolution phase under REDOR,

$$
\Delta \Phi\left(N t_{r}\right)=\overline{\omega_{D}}\left(\alpha, \beta, t_{r}\right) \times N t_{r}=\frac{\gamma_{I} \gamma_{S} \eta \mu_{0}}{4 \pi r^{3}} \frac{1}{2} \frac{\sqrt{2}}{\pi} \sin 2 \beta \sin \alpha \times N t_{r} .
$$


The scaling factor resulted from recoupling the dipolar coupling by REDOR under MAS is $\frac{\sqrt{2}}{\pi} \approx 0.45$

The observed dipolar dephasing

equals $S / S_{0}\left(N t_{r}\right)=\left\langle\cos \Delta \Phi\left(N t_{r}\right)\right\rangle=\iint \cos \left[\Delta \Phi\left(\alpha, \beta, N t_{r}\right)\right] \sin \beta d \beta d \alpha$

a)

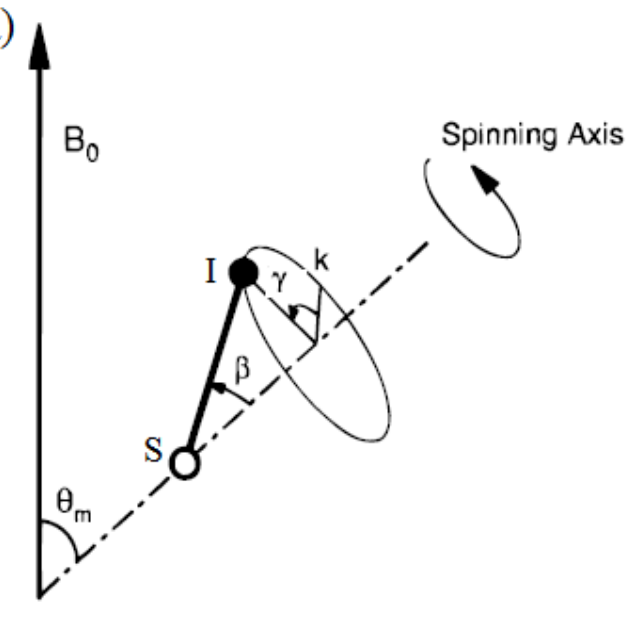

b)

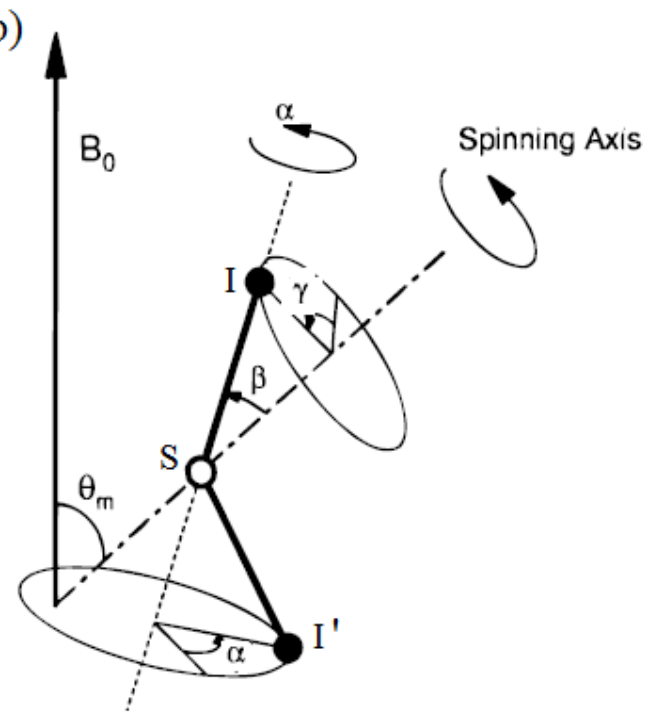

Figure 1.A3. Definitions of the angles used in the calculation of dipolar dephasing in REDOR. a) An isolated heteronuclear dipolar coupling vector under rotation around the rotor axis $\left(\theta_{\mathrm{m}}=54.7^{\circ}\right.$ with $\left.\mathrm{B}_{0}\right)$. The azimuthal angle $\gamma$ is measured between the reference vector $\mathrm{k}$ within the plane of $\mathrm{B}_{0}$ and the rotor axis and $\beta$ is the angle between the dipolar vector and the rotor axis. b) For a single spin interacting with multiple heteronuclear spins, a third angle $\gamma$ is needed to describe all the orientations, and $\alpha$ defines the rotation about a common chosen internuclear vector.

For the spin systems of interest in our research, for instance, every citrate carbon interacts and is dephased by multiple ${ }^{31} \mathrm{P}$ spins in apatite, multi-spin analysis is more realistic and accurate. For a collection of I-spins dipolar coupled to an observed S-spin, three angles are required to describe all possible orientations of the intermolecular vectors in a spinning powder sample, as defined in Figure 1.A3. This can be simplified by rotating the $\mathrm{B}_{0}$ field 
around the rotor axis with a fixed angle of $54.7^{\circ}$. The polar coordinates for $\mathrm{B}_{0}$ are $\left(54.7^{\circ}, 0\right)$ and for nth I-S intermolecular vector $\left(\beta_{n}, \alpha_{n}\right)$.

$$
\begin{aligned}
& \omega_{n}\left(\beta_{n}, \gamma_{n}, r\right)=\frac{\gamma_{I} \gamma_{S} \eta \mu_{0}}{4 \pi r^{3}} \frac{1}{2} \frac{\sqrt{2}}{\pi} \sin 2 \beta_{n} \sin \gamma_{n} . \\
& S / S_{0}=\left\langle\prod_{n} \cos \Delta \Phi_{n}\right\rangle=\iiint \prod_{n} \cos \left[\Delta \Phi_{n}\left(\beta_{n}, \gamma_{n}, r\right)\right] \sin \beta d \beta d \gamma d r
\end{aligned}
$$

For the initial decay $\Delta \Phi<<1$,

$\prod_{n} \cos \Delta \Phi_{n} \approx \prod_{n}\left(1-\Delta \Phi_{n}^{2} / 2\right) \approx \prod_{n} \exp \left(-\Delta \Phi_{n}^{2} / 2\right)=\exp t\left(-\sum_{n} \Delta \Phi_{n}^{2} / 2\right)=\exp \left(-\sum_{n} \omega_{n}^{2} t^{2} / 2\right)$

The dephasing can be evaluated by simulations. The simulation requires knowledge of the relative geometry of the subject. The REDOR decay of multi-spin systems can be analyzed using available packages such as SIMPSON ${ }^{55}$ or SPINEVOLUTION ${ }^{56}$. Alternatively, since the initial part of the dipolar evolution in REDOR proves to be nearly geometry-independent ${ }^{57}$ (eq. 1.A10), the data can be analyzed based solely on distances. Also, Gullion and Pennington have proposed $\theta$-REDOR method, which reduces the multispin problem to a spin-pair on a probabilistic basis ${ }^{58}$. However, this approach results in an attenuation of the overall effects.

Other interactions interfering with dipolar coupling measurement in REDOR have also been discussed. Weak homonuclear couplings are removed with fast MAS, while for strong ones as in ${ }^{1} \mathrm{H}$ - or ${ }^{19} \mathrm{~F}$-rich spin systems, they commute with relatively weak heteronuclear couplings. J-couplings are not affected by MAS and exist in uniformly labeled samples, but in organic solids they are much weaker than dipolar couplings and can be removed by selective-REDOR ${ }^{59}$. Weak homonuclear J-couplings can sometimes interfere with the REDOR measurement of long-distance weak dipolar couplings. However, the coherence term of homonuclear J-couplings have the form of $\mathrm{I}_{\mathrm{iz}} \mathrm{I}_{\mathrm{j} z}$, so selective inversion of one of the I-spins in the middle of the recoupling period renders the sign of the J-evolution phase during the second half of the recoupling period the same as the first half but with an oppiste sign. Thus, the homonulcear J-coupling is refocused at the end of the complete 
recoupling period, while the weak heteronuclear coupling with the form of $\mathrm{I}_{\mathrm{Z}} \mathrm{S}_{\mathrm{Z}}$ is not affected by the selective pulse.

\section{HeteronucleAr recoupling with dephasing from strong homonuclear interactions of} protons (HARDSHIP). Different from REDOR, HARDSHIP ${ }^{26}$ recouples the heteronulcear interaction between $\mathrm{X}$-nuclei and short- $\mathrm{T}_{2}$ protons only; this difference is essential in determining the thickness of nanoparticles in organic-inorganic nanocomposites such as bone and bone-mimicking nanocomposites.

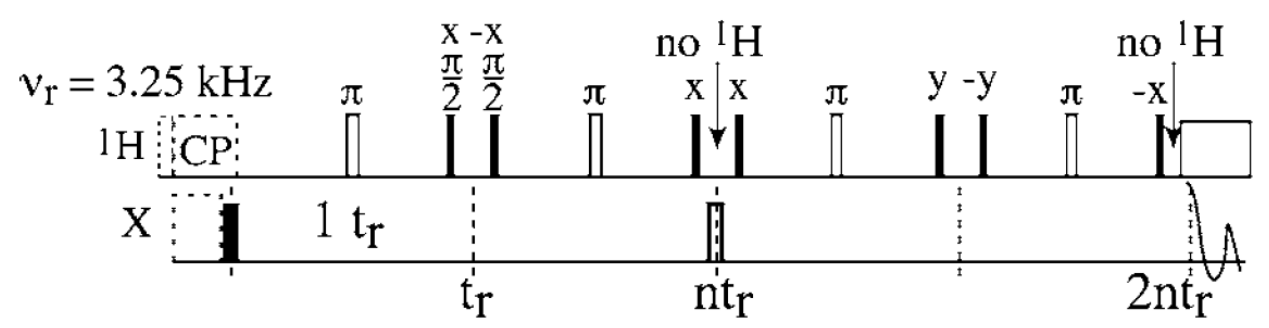

Figure 1.A4. HARDSHIP pulse sequence suitable for slow MAS. At fast MAS, the REDOR recoupling and ${ }^{1} \mathrm{H}$ transverse dephasing periods can be extended to multiple rotor periods. For ${ }^{1} \mathrm{H}$ chemical shifts of a large dispersion, a $\pi$-pulse can be implemented in the middle of every ${ }^{1} \mathrm{H} \mathrm{T}_{2}$ dephasing period. Figure adapted from Schmidt-Rorh et al. ${ }^{26}$

In HARDSHIP, the ${ }^{1} \mathrm{H}-\mathrm{X}$ heteronuclear interaction is recoupled under MAS with a REDOR sequence. Without a $\pi$-pulse in the middle as in REDOR $S_{0}$, the heteronuclear interactions between the $\mathrm{X}$ nuclei and long- $\mathrm{T}_{2}$ protons are refocused at the beginning of detection, while the multi-quantum coherence of the $\mathrm{X}$-nuclei and the short- $\mathrm{T}_{2}$ protons vanishes before the second recoupling period of REDOR due to fast $\mathrm{T}_{2}$ relaxation; thus they REDOR dephasing of the $\mathrm{X}$-nuclei by short- $\mathrm{T}_{2}$ protons is not refocused. Therefore, unlike REDOR, the measured HARDSHIP dephasing of the X-nuclei is solely by short- $\mathrm{T}_{2}$ protons rather than all protons. This grants HARDSHIP the special capability of characterizing the thickness of nanoparticles such as phoshphates and carbonates in bone and silicates in polymers, because without dephasing by the protons of long- $T_{2}$ within the nanoparticles. The dephasing rate by the polymer "short- $\mathrm{T}_{2}$ " protons has a strong dependence on the thickness 
of the inorganic particle. The numerical simulation to fit the HARDSHIP decay curve is analogous to multispin REDOR simulations ${ }^{26}$.

Centerband-only detection of exchange (CODEX). CODEX $^{6061,62}$ is a high resolution method to detect slow motion inducing molecular reorientation and spin exchange on the time scale of $0.1 / \mathrm{s}-5,000 / \mathrm{s}$. The motional amplitude, the number of exchanging magnetically inequivalent sites, as well as the average molecular distances involved in the spin exchange process, can be quantified with analytical and numerical simulations in conjunction with experimental measurements. The slow motion process sometimes is tangled with spin diffusion; nevertheless, the spin diffusion can be minimized with fast MAS ${ }^{63}$ and motion can be reduced with low temperature measurements.

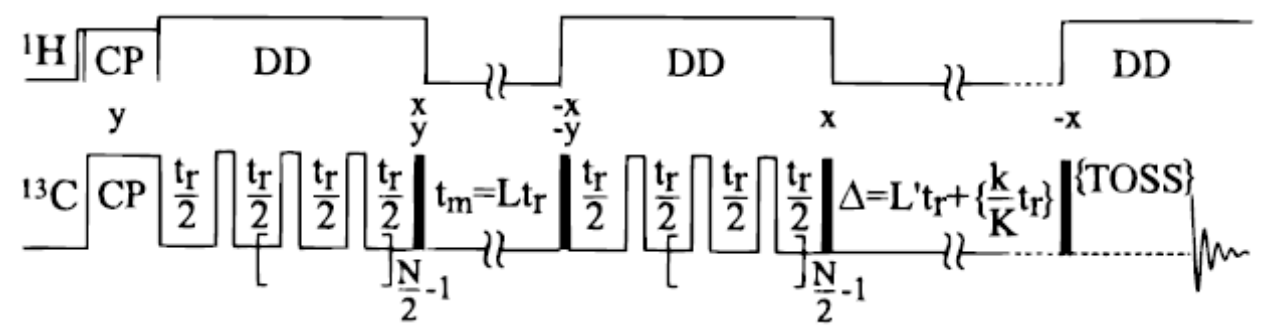

Figure 1.A5. CODEX pulse sequence. Filled rectangles are $\pi / 2$ pulses and non-filled are $\pi$ pulses. The $\mathrm{t}_{\mathrm{m}}$ and $\Delta$ periods are exchanged for measurements of $\mathrm{S}$ and $\mathrm{S}_{0}$ (reference signal for compensation of $\mathrm{T}_{1}$ relaxation). $\gamma$-integral and TOSS are employed for obtaining absorptive and sideband-free spectra. Figure adapted from deAzevedo et al. ${ }^{61}$

In CODEX, the chemical shift anisotropy is recoupled by a train of $\pi$-pulses with each pulse placed at every half rotor period just as in REDOR. After a mixing period $t_{m}$, another train of $\pi$-pulses are applied. If there's no motional reorientation or spin exchange occurs, a stimulated echo is generated and detected. Otherwise, the dephasing of the original magnetization is observed. The $\mathrm{T}_{1}$ relaxation effect is compensated by switching the $\mathrm{t}_{\mathrm{m}}$ and $\Delta$ periods. TOSS can be implemented with $\gamma$-integral for efficient sideband removal. The dephasing factor $\mathrm{D}\left(\mathrm{t}_{\mathrm{m}}, \delta \mathrm{Nt}_{\mathrm{r}}\right)$ is calculated as 


$$
\begin{aligned}
& D\left(t_{m}, \delta N t_{r}\right)=\frac{S\left(t_{m}, \delta N t_{r}\right)}{S\left(0, \delta N t_{r}\right)}=\operatorname{Re}\left(\left\langle\exp \left(i\left(\Phi_{1}+\Phi_{2}\right)\right)\right\rangle=\operatorname{Re}\left(\left\langle\exp \left(i\left(\left|\Phi_{1}\right|+\left|\Phi_{2}\right|\right)\right)\right\rangle\right.\right. \\
& =\operatorname{Re}\left\langle\operatorname { e x p } \left( i \int_{0}^{t_{r} / 2}\left(N \omega_{2}(t)-N \omega_{1}(t) d t\right\rangle=\operatorname{Re}\left\langle\exp \left(i \Phi^{\Delta}\left(\delta N t_{r}, \alpha_{R}, \beta_{R}, \gamma_{R}\right)\right)\right\rangle,\right.\right.
\end{aligned}
$$

where $\omega_{1}$ and $\omega_{2}$ are the anisotropic evolution frequencies before and after the mixing time. The dephasing only depends on the total dephasing time $\mathrm{Nt}_{\mathrm{r}}$, not the spinning speed.

$$
\Phi^{\Delta}=N\left(\Phi_{1}\left(\frac{t_{r}}{2}\right)-\Phi_{2}\left(\frac{t_{r}}{2}\right)\right)=N t_{r} \frac{\delta}{2 \pi}\left(\chi_{1}\left(\frac{t_{r}}{2}\right)-\chi_{2}\left(\frac{t_{r}}{2}\right)\right),
$$

where $\chi_{n}\left(\frac{t_{r}}{2}\right)=\left(\tilde{S}_{1}\left(\alpha_{n}, \beta_{n}, \gamma_{n}\right)-\frac{1}{2} \tilde{S}_{2}\left(\alpha_{n}, \beta_{n}, \gamma_{n}\right)\right) / \delta$, with $\tilde{S}_{n}$ defined in ref. ${ }^{64}$. For uniaxial interactions $(\eta=0)$, a master curve of $\mathrm{D}\left(\mathrm{t}_{\mathrm{m}}, \delta \mathrm{Nt}_{\mathrm{r}}\right)=\mathrm{f}\left(1.5 \delta \mathrm{Nt}_{\mathrm{r}} \sin \beta_{\mathrm{R}}\right)$ can be derived.

Otherwise, the shape of the curve also depends on other orientation angles and no master curve exists. For uniaxial interactions with an orientation angle distribution $R\left(\beta_{R}, t_{m}\right)$, the dephasing factor $\mathrm{D}\left(\mathrm{t}_{\mathrm{m}}, \delta \mathrm{Nt}_{\mathrm{r}}\right)$ can be expressed as

$$
D\left(t_{m}, \delta N t_{r}\right)=1-\int_{0^{\circ}}^{90^{\circ}} R\left(\beta_{R} ; t_{m}\right) \varepsilon\left(\delta N t_{r} ; \beta_{R}\right) d \beta_{R}
$$

where $\varepsilon\left(\delta \mathrm{Nt}_{\mathrm{r}} ; \beta_{\mathrm{R}}\right)$ is the normalized pure exchange intensity.

The final dephased intensity is

$$
D_{\infty}=D\left(t_{m}>>\tau_{c}, \delta N t_{r}>>1\right)=1-\left(1-\frac{1}{M}\right) f_{m}
$$

where $\mathrm{M}$ is the number of accessible chemically equivalent magnetically inequivalent sites and $\mathrm{f}_{\mathrm{m}}$ is the fraction of mobile components.

CODEX not only can be used to determine the reorientation angles and their distributions, number of exchanging magnetically inequivalent sites $M$, and the fraction of mobile moieties $\mathrm{f}_{\mathrm{m}}$, but is also a method to resolve distances among molecules based on spin exchange theory, as used in this thesis to analyze the citrate density on the apatite surface in bone.

During spin exchange among $\mathrm{n}$ spins, the time evolution of the magnetization can be characterized by 
$\frac{d \vec{M}(t)}{d t}=-\overleftrightarrow{\Pi} \vec{M}(0)$,

with $\overleftrightarrow{\Pi}$ as the spin exchange matrix composed of off-diagonal elements

$\Pi_{m n}=0.5 \pi \omega_{m n}^{2} F_{m n}(0)$

where $\omega_{\mathrm{mn}}$ is the homonuclear dipolar coupling frequency calculated as

$\omega_{m n}=\frac{\mu_{0}}{4 \pi} \gamma^{2} \eta \frac{1}{r_{m n}^{3}} \frac{1}{2}\left(3 \cos ^{2} \theta_{m n}-1\right)$

$\omega_{\mathrm{mn}}$ depends on both the distance between two nuclei and the angle $\theta$ of the dipolar coupling vector relative to the magnetic field. The angle $\theta$ becomes time-dependent under MAS, which is a function of angle $\beta$ between the dipolar vector and the rotor axis and the rotation angle $\gamma$. The $\beta$-averaged value of $\left\langle\left(3 \cos ^{2} \theta_{m n}-1\right)^{2}\right\rangle$ is 0.2 .

$\mathrm{F}_{\mathrm{mn}}(0)$ is the overlap integral:

$$
F_{m n}(0)=\int_{-\infty}^{+\infty} f_{m}\left(\omega-\omega_{m}\right) f_{n}\left(\omega-\omega_{n}\right) d \omega,
$$

where $\mathrm{f}$ is the normalized single-quantum line shape without proton decoupling. The overlap integral represents the possibility of single-quantum transition occurring at the same frequency for both spin $\mathrm{m}$ and $\mathrm{n}$; its magnitude is influenced by isotropic chemical shifts, CSA, $\mathrm{X}-{ }^{1} \mathrm{H}$ heteronuclear dipolar couplings, and ${ }^{1} \mathrm{H}-{ }^{1} \mathrm{H}$ homonuclear dipolar couplings. The value of $F_{m n}(0)$ can be calibrated with model compounds or with known dipolar distances within the investigated system ${ }^{65}$.

Since the sum of each column in $\Pi_{m n}$ has to be zero, the diagonal element is the sum of the off-diagonal elements of the same column with a negative sign.

The normalized CODEX intensity $\mathrm{S} / \mathrm{S}_{0}$ is the fraction of the magnetization still residing on the original spin after spin exchange, which can be calculated from the magnetization evolution expression,

$\vec{M}(t)=\exp (\overleftrightarrow{\Pi} t) \vec{M}(0)$

For $\vec{M}(0)=\delta_{\mathrm{mn}}$, describing the initial magnetization only on the nth spin, the CODEX intensity 
$S / S_{0}=\sum_{n}\left(\exp (\overleftrightarrow{\Pi} t) \mathrm{e}_{\mathrm{n}}\right)_{\mathrm{n}}=\operatorname{tr}(\exp (\overleftrightarrow{\Pi} t)$

Wideline separation (WISE). The narrow $(\sim 10 \mathrm{ppm}){ }^{1} \mathrm{H}$ spectral range combined with peaks of broad linewidth due to strong ${ }^{1} \mathrm{H}$ homonuclear dipolar couplings in solid state NMR results in severely overlapped peaks. Thus, information such as molecular dynamics is difficult to extract from unresolved peaks. Wideline separation (WISE) NMR integrates ${ }^{1} \mathrm{H}$ 1D NMR, cross polarization (CP), ${ }^{13} \mathrm{C}$ NMR, and MAS to separate wideline ${ }^{1} \mathrm{H}$ spectra by encoding the ${ }^{1} \mathrm{H}$ information into the ${ }^{13} \mathrm{C}$ detected spectra with a larger spectral range ${ }^{66}$. Fourier transformation allows separating ${ }^{1} \mathrm{H}$ peaks according to their correlation to different carbons, thus knowledge on the dynamics of individual components can be obtained from the resolved ${ }^{1} \mathrm{H}$ spectra with distinct linewidth.

a)

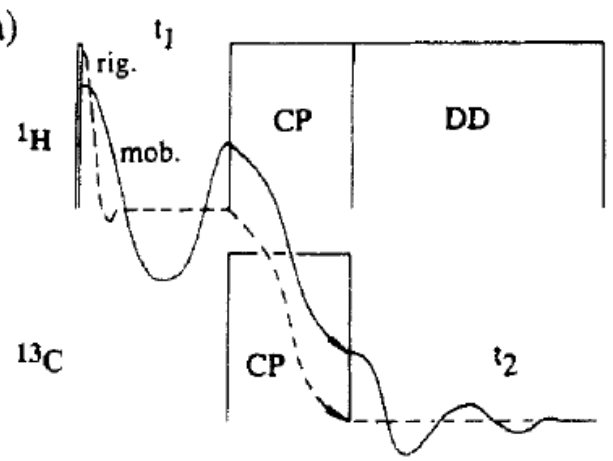

b)

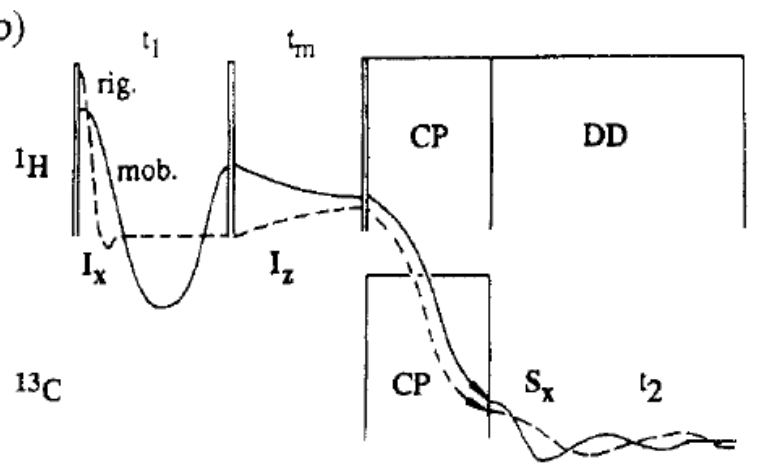

Figure 1.A6. Pulse sequence of WISE NMR. a) Basic version, b) WISE NMR with ${ }^{1} \mathrm{H}$ spin diffusion. Figure adapted from Schmidt-Rohr et al. ${ }^{66}$

WISE with ${ }^{1} \mathrm{H}$ spin diffusion probes the proximity of different segments manifested by the ${ }^{1} \mathrm{H}$ lineshape change with increasing the mixing time $\mathrm{t}_{\mathrm{m}}$. The spin diffusion equilibrium is reflected in the equal lineshapes of all components in the ${ }^{1} \mathrm{H}$ dimension of the 2D WISE spectra. The mixing time needed to reach equilibrium can be used to confirm the nanocomposite formation and estimate the domain size provided with the information of the ${ }^{1} \mathrm{H}$ densities in heterogeneous systems as described in reference ${ }^{43}$. 
For the purpose of separating ${ }^{1} \mathrm{H}$ spectra via WISE, spin diffusion during $\mathrm{CP}$ is undesirable, which is usually removed by Lee-Goldberg $\mathrm{CP}$ with the effective magnetic field making the magic angle with the $\mathrm{z}$ direction.

$\mathrm{A}^{1} \mathrm{H}^{-13} \mathrm{C}$ correlation experiment can also be implemented with ${ }^{1} \mathrm{H}$ homonuclear decoupling using techniques such as frequency-switched Lee-Goldberg (FSLG) and MREV8 , during $\mathrm{t}_{1}$ evolution to obtain high resolution ${ }^{1} \mathrm{H}$ spectra in the $\omega_{1}$ dimension for identification of isotropic ${ }^{1} \mathrm{H}$ chemical shifts.

Spectral editing. Spectral editing techniques aim to separate ${ }^{13} \mathrm{C}$ signals of quaternary carbon, $\mathrm{CH}, \mathrm{CH}_{2}$, and $\mathrm{CH}_{3}$ and are proven to be very important in organic matter characterization. The selection of quaternary carbon can be achieved via gated dipolar decoupling or recoupling, which eliminates signals from rigid $\mathrm{CH}_{\mathrm{n}}$. $\mathrm{CH}_{3}$ usually rotate very fast with a relatively short ${ }^{13} \mathrm{C}_{1}$, thus it can be singled out by a short recycle delay. However, the selection of $\mathrm{CH}$ and $\mathrm{CH}_{2}$ signals is not trivial.

CH selection. The $\mathrm{CH}$ signals are mainly tangled with those of $\mathrm{CH}_{2}$ in ${ }^{13} \mathrm{C}$ spectra. The difference between $\mathrm{CH}$ and $\mathrm{CH}_{2}$ is the number of protons they are coupled to; $\mathrm{CH}$ only has one proton and the multiple-quantum (MQ) coherence commutes with $\mathrm{CH}$ dipolar coupling Hamiltonian, $\left[\mathrm{I}_{x / y} \mathrm{~S}_{\mathrm{x} / \mathrm{y}}, \mathrm{I}_{\mathrm{z}} \mathrm{S}_{z}\right]=0$ so the $\mathrm{MQ}$ evolution is unchanged under $\mathrm{CH}$ dipolar coupling, while the carbon in $\mathrm{CH}_{2}$ is exposed to the field of two protons, and $\left[\mathrm{I}_{\mathrm{x} / \mathrm{y}, \mathrm{A}} \mathrm{S}_{\mathrm{x} / \mathrm{y}}, \mathrm{I}_{\mathrm{z}, \mathrm{B}} \mathrm{S}_{\mathrm{z}}\right]$ $\neq 0$, so the MQ coherence evolves under $\mathrm{CH}$ dipolar coupling. Therefore, $\mathrm{CH}_{2}$ signals can be removed by exciting MQ coherence and letting it evolve under the $\mathrm{CH}$ dipolar coupling. The following pulse sequence is used for $\mathrm{CH}$ selection ${ }^{67}$. 


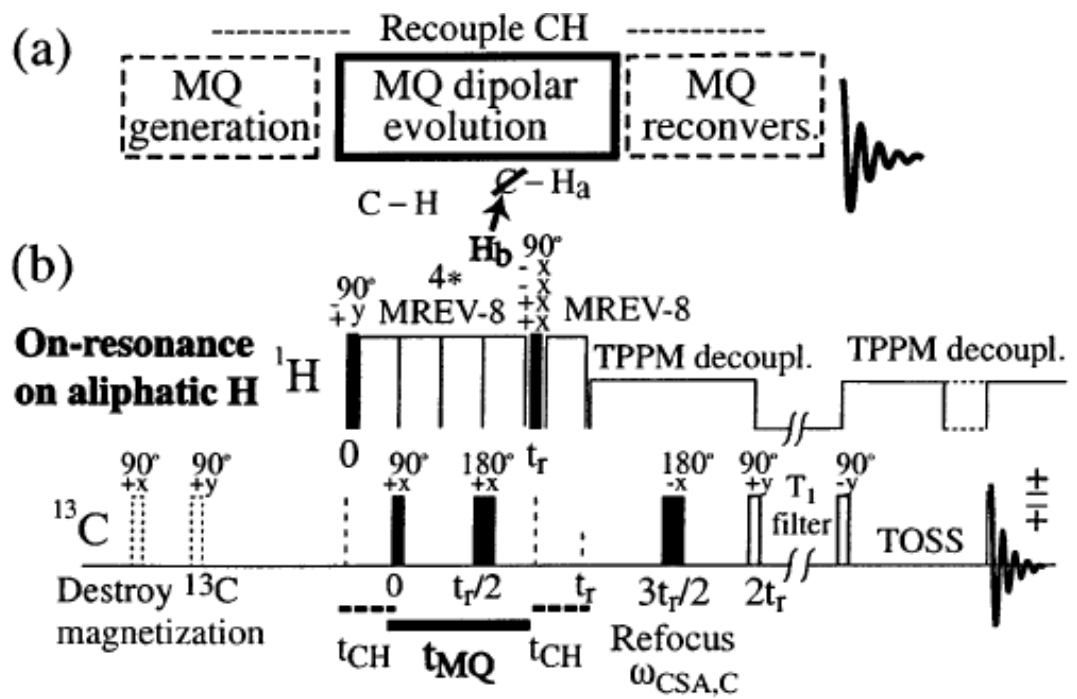

Figure. 1.A7. Block diagram (a) and pulse sequence for $\mathrm{CH}$ suppression. Figure adapted from Schmidt-Rohr et al. ${ }^{67}$

The residual $\mathrm{CH}_{3}$ coherence and quaternary carbons can be further removed by $\mathrm{T}_{1}$ filter and inverse gated decoupling. This experiment is usually performed at 4 or $5.8 \mathrm{kHz}$ with an efficiency of $14 \%$.

$\mathrm{CH}_{2}$ selection. $\mathrm{CH}_{2}$ selection is accomplished by manipulating the spin terms using the following pulse sequence. Only the three spin term $\mathrm{S}_{\mathrm{x}} \mathrm{I}_{\mathrm{Z}} \mathrm{J}_{\mathrm{Z}}$ from $\mathrm{CH}_{2}$ is retained and converted to observables $\mathrm{S}_{\mathrm{x}} \cdot{ }^{13} \mathrm{C}$ chemical shift evolution is refocused by $180^{\circ}$ pulses, and $\mathrm{CH}_{3}$ signals are removed by $\mathrm{T}_{1}$ filter. Three spin coherence from long-range dipolar coupling to $\mathrm{CH}$ to another ${ }^{1} \mathrm{H}$ is compensated by setting $\beta>90^{\circ} .68$

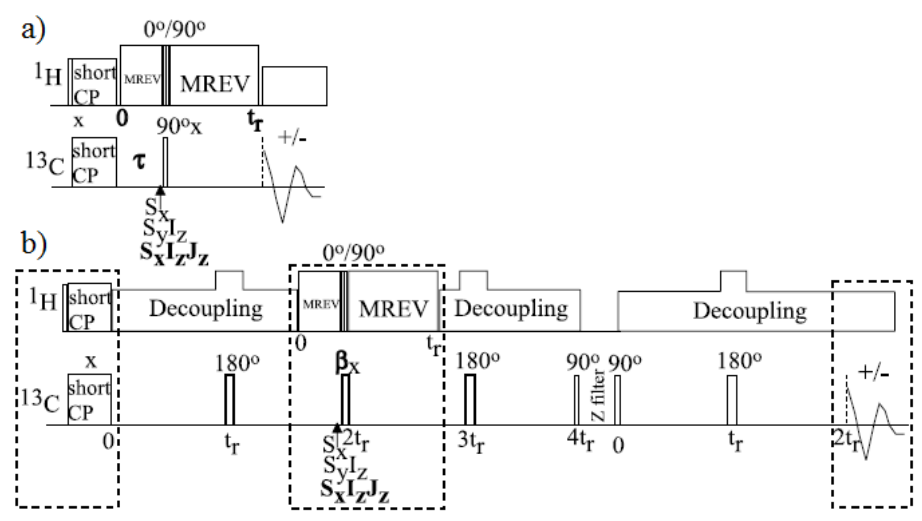

Figure 1.A8. Pulse sequence for $\mathrm{CH}_{2}$ selection. a) Essential pulse block for $\mathrm{CH}_{2}$ selection. Short $\mathrm{CP}$ is used for reducing quaternary and $\mathrm{CH}_{3}$ signal intensity. b) The full pulse sequence 
with ${ }^{13} \mathrm{C}$ chemical shift refocusing by multiple $\pi$-pulses and the $\mathrm{CH}_{3}$ signal intensity is further reduced by implementing a Z-filter. Figure adapted from Mao et al. ${ }^{68}$

Dipolar chemical shift (DIPSHIFT) correlation. Dipolar chemical shift (DIPSHIFT) is a separate-local field experiment ${ }^{69}$. It measures the heteronuclear dipolar coupling strength related to a particular isotropic chemical shift. The reduction of the measured dipolar coupling with respect to the rigid limit reveals the presence of local motions on the microsecond scale.

a)

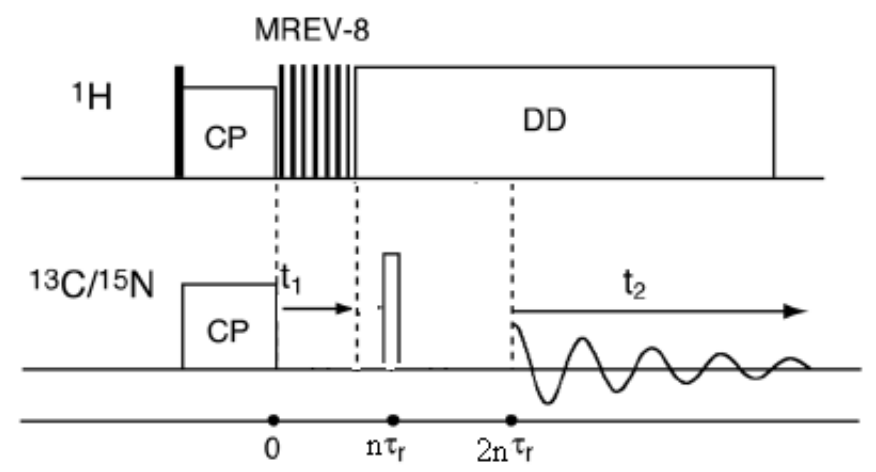

b)

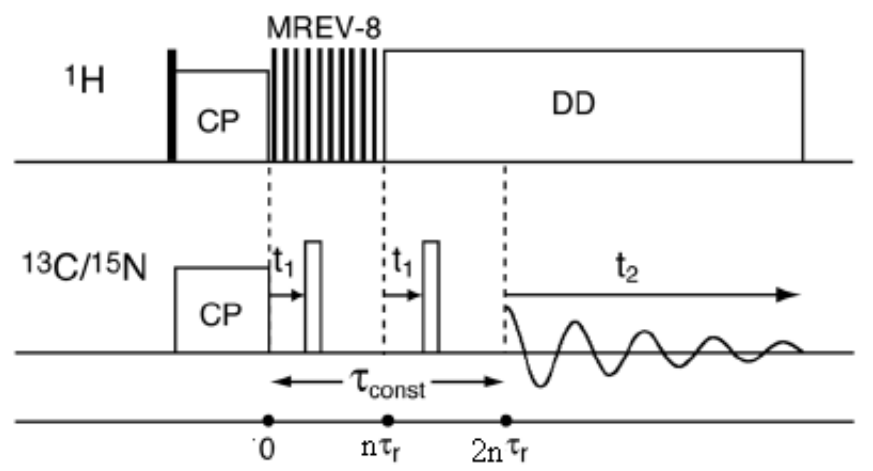

Figure 1.A9. Pulse sequence of DIPSHIFT. a) original version of DIPSHIFT. b) "Double"DIPSHIFT for enhancement of $\mathrm{H}-\mathrm{X}$ dipolar coupling measurement.

In the original DIPSHIFT pulse sequence, after $\mathrm{CP}$, the magnetization of the $\mathrm{X}$ nuclei evolves with ${ }^{1} \mathrm{H}-\mathrm{X}$ heteronuclear dipolar coupling under MAS and ${ }^{1} \mathrm{H}$ homonuclear decoupling (by techniques such as Mansfield-Rhim-Elleman-Vaughan-8 pulses (MREV-8), Frequency Switched Lee-Goldberg (FSLG), or Waugh-Huber-Haeberlen (WAHUHA)). The ${ }^{1} \mathrm{H}$-X heteronuclear interaction is decoupled after the $t_{1}$ evolution period. The isotropic 
chemical shift evolution of the $\mathrm{X}$ nuclei is refocused at the beginning of detection $\left(\mathrm{t}_{2}=0\right)$ by a $\pi$-pulse positioned at $\mathrm{nt}_{\mathrm{r}}$. Fourier transformation of the 2D DIPSHIFT spectrum gives ${ }^{1} \mathrm{H}-\mathrm{X}$ dipolar coupling pattern in the $\omega_{1}$ dimension and chemical shift of $X$ nuclei in the $\omega_{2}$ dimension. Information including internuclear distance, relative tensor orientations (at slow MAS), and the amplitude of motions (order parameter) can be extracted. Since the intensity of the detected signal is modulated by ${ }^{1} \mathrm{H}-\mathrm{X}$ dipolar evolution, the measurement of ${ }^{1} \mathrm{H}-\mathrm{X}$ dipolar coupling constant can be achieved by analyzing time-domain signal decay with increasing $\mathrm{t}_{1}$ dipolar evolution time or the dipolar coupling pattern in $\omega_{1}$ of the $2 \mathrm{D}$ spectra after Fourier transformation.

Weak heteronuclear dipolar coupling measurements can be enhanced by applying multiple $\pi$-pulses to accumulate the dipolar coupling evolution phase $\Phi=\mathrm{f}\left(\mathrm{n} \phi\left(\mathrm{t}_{1}\right)\right.$, which is particularly useful for determination of small dipolar coupling constant under fast MAS ${ }^{70}$.

Chemical shift anisotropy (CSA) measurement. Chemical shift anisotropy is often undesirable in high resolution NMR and spun out by fast MAS; nevertheless, it contains rich information on the local environment, the orientation, and the dynamics of the molecules. Actually, a difference in CSA can add another dimension to distinguish chemical species of the same isotropic chemical shift, as used in CSA filter experiments. ${ }^{71}$

CSA is refocused at the end of every rotor period under MAS; to recouple the CSA interaction, a $\pi$-pulse can be placed before the end of a full rotor period. Maximum CSA evolution within one $t_{r}$ can be achieved when the $\pi$-pulse is positioned at $t_{r} / 2$. Elegant modifications of this simple scheme have been proposed as described below to simplify data analysis, obtain absorptive spectra, and enhance the CSA interaction measurement by accumulating the CSA evolution phase over multiple rotor periods employing multiple $\pi$ pulses $^{71}$. 
(a)
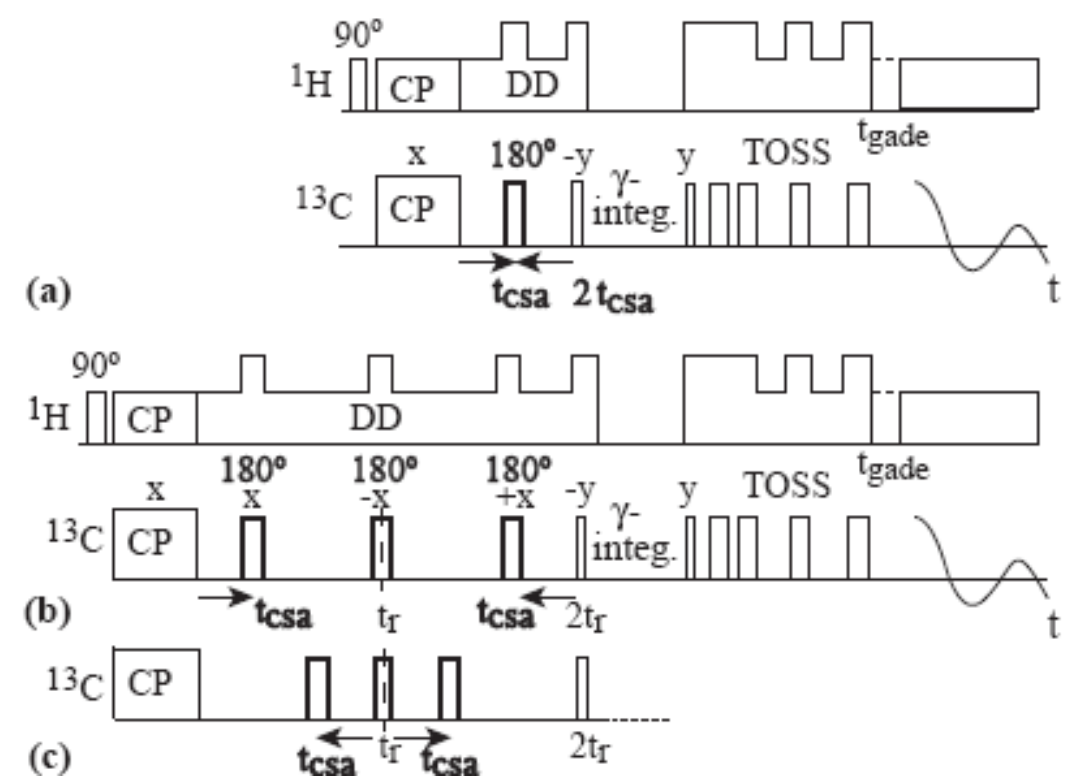

Figure 1.A10. Pulse sequences for CSA dephasing measurement. a) Three-pulse sequence with a $\pi$-pulse in the middle of the CSA dephasing period $2 \mathrm{t}_{\mathrm{CSA}}$ generating a Hahn-echo to refocus the isotropic shift evolution. Two $\pi / 2$ pulses retain only the cosine component of the anisotropic phase evolution. A $\gamma$-integral is implemented to produce absorptive clean spectra. TOSS is used to remove spinning sidebands. b) Five-pulse sequence to enhance CSA dephasing measurement, good for very long and short dephasing time. c) Equivalent as in b), suitable for intermediate dephasing time. Figure adapted from Mao et al. ${ }^{71}$

With the three-pulse sequence, the total CSA evolution phase during $2 \mathrm{t}_{\mathrm{CSA}}$ can be calculated as

$$
\Phi_{t o t}\left(2 t_{C S A}\right)=\int_{0}^{t_{C S A}} \omega(t) d t-\left(\int_{0}^{2 t_{C S A}} \omega(t) d t-\int_{0}^{t_{C S A}} \omega(t) d t\right)=2 \Phi\left(t_{C S A}\right)-\Phi\left(2 t_{C S A}\right) .
$$

The dephasing factor is

$$
S / S_{0}=\left\langle\cos \left(\Phi_{t o t}\left(2 t_{C S A}\right)\right)\right\rangle \text {, }
$$

where $S_{0}$ is measured with $t_{C S A}=0$ and the bracket represents powder averaging. Similarly, with the five-pulse sequence, 


$$
\begin{aligned}
& \Phi_{t o t}\left(2 t_{r}, t_{C S A}\right)=-\int_{0}^{t_{C S A}} \omega(t) d t+\left(\int_{0}^{t_{r}} \omega(t) d t-\int_{0}^{t_{C S A}} \omega(t) d t\right)-\left(\int_{0}^{2 t_{r}} \omega(t) d t-\int_{0}^{t_{C S A}} \omega(t) d t\right)+\left(\int_{0}^{2 t_{r}} \omega(t) d t-\int_{0}^{2 t_{r}-t_{C S A}} \omega(t) d t\right) \\
& =-2 \Phi\left(t_{C S A}\right)+2 \Phi\left(-t_{C S A}\right)
\end{aligned}
$$

and

$$
S / S_{0}=\left\langle\cos \left(\Phi_{t o t}\left(2 t_{r}, t_{C S A}\right)\right)\right\rangle \text {. }
$$

The CSA dephasing is largely determined by $\delta_{\mathrm{CSA}}$ and has only a weak dependence on the asymmetry parameter $\eta$.

The CSA parameters can be determined either from the analysis of the time-domain signal decay due to CSA evolution, or powder pattern or spinning sideband analysis in the frequency domain ${ }^{72}$.

To preserve CSA while still retaining the benefits of high resolution NMR, various methods, such as separation of undistorted powder patterns by effortless recoupling (SUPER) ${ }^{73}$, phase-adjusted spinning sidebands (PASS) ${ }^{53}$, and magic angle turning (MAT) ${ }^{46}$, have been developed. Among these techniques, MAT has been recognized as the best broadband method due to its use of only short $\pi / 2$ - pulses; MAT has been adapted to fast spinning speed to enhance signal sensitivity and demonstrated on various tellurides with broad spectral range as described in Chapter 5 . The technical aspects including off-resonance effects, sharing between channels after shearing as well as echo-matched Gaussian filtering are detailed in Chapter 6.

Multi-echo refocused (or Carr-Purcell-Meiboom-Gill) detection. Refocused detection (or CPMG) ${ }^{74}$ is used to enhance signal sensitivity by applying multiple Hahn-echo sequences to sustain the NMR signal and allow the decay with the time constant of $T_{2}$ instead of much shorter $\mathrm{T}_{2}{ }^{*}$. This method is particularly useful for nuclei with $\mathrm{T}_{2} \gg \mathrm{T}_{2}{ }^{*}$, such as isolated ${ }^{31} \mathrm{P}$, ${ }^{29} \mathrm{Si}$, and ${ }^{13} \mathrm{C}$ spins. The factor of signal enhancement is $\sqrt{T_{2} / T_{2} *}$. Chemical shift resolution is compromised as a consequence of using refocused detection.

The receiver is blanked during the pulses and the following dead time. The noise from radio frequency pulses is filtered out by saturating the receiver with a larger than 
regular receiver gain $(\mathrm{RG})$, such as $16 \mathrm{k}(16 \mathrm{x} 1024)$ on a Bruker NMR spectrometer. The ${ }^{1} \mathrm{H}$ decoupling power level is reduced for a long acquisition time of $\sim 100 \mathrm{~ms}$ to the extent possible in the specific spin system.

a)

b)


Figure 1.A11. Refocused multi-echo detection. a) Pulse sequence for refocused detection (or CPMG). b) Demonstration of refocused detection on a ${ }^{13} \mathrm{C}$ spectrum of bovine bone. Thick red line: ${ }^{13} \mathrm{C}$ spectrum acquired with normal detection; thin black line: with refocused detection.

The distance between two spikelets in spectra obtained with refocused detection is $1 / 2 \tau$ ( $\tau$ is usually chosen as $\mathrm{nt}_{\mathrm{r}}$ ). The peak of interest is set on resonance or exactly one spikelet away from on resonance. If two or more peaks of interest, the distance between two neighboring spikelets is chosen as a common divider of the difference in peak positions in the normal detected spectra.

\section{References}

1. Rey, C.; Combes, C.; Drouet, C.; Glimcher, M. J., Bone mineral: update on chemical composition and structure (vol 20, pg 1013, 2009). Osteoporos Int 2009, 20, (12), 2155-2155.

2. Currey, J. D., Bones. Princeton University Press: 2002; p 456.

3. Weiner, S.; Wagner, H. D., The material bone: Structure mechanical function relations. Аnпu. Rev. Mater. Sci. 1998, 28, 271-298. 
4. Buckwalter, J. A.; Glimcher, M. J.; Cooper, R. R.; Recker, R., Bone Biology .1. Structure, Blood-Supply, Cells, Matrix, and Mineralization. J Bone Joint Surg Am 1995, 77A, (8), 1256-1275.

5. Weiner, S.; Traub, W., Bone-Structure - from Angstroms to Microns. FASEB J. 1992, $6,(3), 879-885$.

6. Currey, J. D., Role of collagen and other organics in the mechanical properties of bone. Osteoporosis International 2003, 14, S29-S36.

7. de Jong, W. F., La substance minerale dans les os. Recl Trav Chim Pays-Bas Belg 1926, $45,445-448$.

8. Rey, C.; Miquel, J. L.; Facchini, L.; Legrand, A. P.; Glimcher, M. J., HydroxylGroups in Bone-Mineral. Bone 1995, 16, (5), 583-586.

9. Cho, G. Y.; Wu, Y. T.; Ackerman, J. L., Detection of hydroxyl ions in bone mineral by solid-state NMR spectroscopy. Science 2003, 300, (5622), 1123-1127.

10. Legros, R.; Balmain, N.; Bonel, G., Age-Related-Changes in Mineral of Rat and Bovine Cortical Bone. Calcified Tissue International 1987, 41, (3), 137-144.

11. Wilson, R. M.; Elliott, J. C.; Dowker, S. E. P.; Rodriguez-Lorenzo, L. M., Rietveld refinements and spectroscopic studies of the structure of Ca-deficient apatite.

Biomaterials 2005, 26, (11), 1317-1327.

12. Lu, H. B.; Campbell, C. T.; Graham, D. J.; Ratner, B. D., Surface characterization of hydroxyapatite and related calcium phosphates by XPS and TOF-SIMS. Analytical Chemistry 2000, 72, (13), 2886-2894.

13. Rey, C.; Collins, B.; Goehl, T.; Dickson, I. R.; Glimcher, M. J., The Carbonate Environment in Bone-Mineral - a Resolution-Enhanced Fourier-Transform InfraredSpectroscopy Study. Calcified Tissue International 1989, 45, (3), 157-164.

14. Eichert, D.; Sfihi, H.; Combes, C.; Rey, C., Specific characteristics of wet nanocrystalline apatites. Consequences on biomaterials and bone tissue. Bioceramics 16 2004, 254-2, 927-930.

15. Grynpas, M. D., Transient precursor strategy or very small biological apatite crystals? Bone 2007, 41, (2), 162-164. 
16. Weiner, S., Transient precursor strategy in mineral formation of bone. Bone 2006, 39, (3), 431-433.

17. Mahamid, J.; Aichmayer, B.; Shimoni, E.; Ziblat, R.; Li, C. H.; Siegel, S.; Paris, O.; Fratzl, P.; Weiner, S.; Addadi, L., Mapping amorphous calcium phosphate transformation into crystalline mineral from the cell to the bone in zebrafish fin rays. Proceedings of the National Academy of Sciences of the United States of America 2010, 107, (14), 6316-6321.

18. Danilchenko, S. N.; Kukharenko, O. G.; Moseke, C.; Protsenko, I. Y.; Sukhodub, L. F.; Sulkio-Cleff, B., Determination of the bone mineral crystallite size and lattice strain from diffraction line broadening. Crystal Research and Technology 2002, 37, (11), 1234-1240.

19. Liu, Y. F.; Manjubala, I.; Schell, H.; Epari, D. R.; Roschger, P.; Duda, G. N.; Fratzl, P., Size and Habit of Mineral Particles in Bone and Mineralized Callus During Bone Healing in Sheep. Journal of Bone and Mineral Research 2010, 25, (9), 2029-2038.

20. Fratzl, P.; Schreiber, S.; Klaushofer, K., Bone mineralization as studied by smallangle x-ray scattering. Connective Tissue Research 1996, 35, (1-4), 9-16.

21. Fratzl, P.; Schreiber, S.; Boyde, A., Characterization of bone mineral crystals in horse radius by small-angle X-ray scattering. Calcified Tissue International 1996, 58, (5), 341-346.

22. Fratzl, P.; Groschner, M.; Vogl, G.; Plenk, H.; Eschberger, J.; Fratzlzelman, N.; Koller, K.; Klaushofer, K., Mineral Crystals in Calcified Tissues - a ComparativeStudy by Saxs. Journal of Bone and Mineral Research 1992, 7, (3), 329-334.

23. Fratzl, P.; Fratzlzelman, N.; Klaushofer, K.; Vogl, G.; Koller, K., Nucleation and Growth of Mineral Crystals in Bone Studied by Small-Angle X-Ray-Scattering. Calcified Tissue International 1991, 48, (6), 407-413.

24. Johansen, E.; Parks, H. F., Electron Microscopic Observations on the 3-Dimensional Morphology of Apatite Crystallites of Human Dentine and Bone. Journal of Biophysical and Biochemical Cytology 1960, 7, (4), 743-\&. 
25. Tong, W.; Glimcher, M. J.; Katz, J. L.; Kuhn, L.; Eppell, S. J., Size and shape of mineralites in young bovine bone measured by atomic force microscopy. Calcified Tissue International 2003, 72, (5), 592-598.

26. Schmidt-Rohr, K.; Rawal, A.; Fang, X. W., A new NMR method for determining the particle thickness in nanocomposites, using T-2,T-H-selective $\mathrm{X}\{\mathrm{H}-1\}$ recoupling. Journal of Chemical Physics 2007, 126, (5), -.

27. Rawal, A. NMR investigations of biological and synthetic phosphate-based nanocomposites. Iowa State University, Ames, 2007.

28. Gao, H. J.; Ji, B. H.; Jager, I. L.; Arzt, E.; Fratzl, P., Materials become insensitive to flaws at nanoscale: Lessons from nature. Proceedings of the National Academy of Sciences of the United States of America 2003, 100, (10), 5597-5600.

29. Klibanski, A.; Adams-Campbell, L.; Bassford, T.; Blair, S. N.; Boden, S. D.; Dickersin, K.; Gifford, D. R.; Glasse, L.; Goldring, S. R.; Hruska, K.; Johnson, S. R.; McCauley, L. K.; Russell, W. E.; Osteopor, N. C. D. P.; Osteoporosis prevention, diagnosis, and therapy. Jama-Journal of the American Medical Association 2001, 285, (6), 785-795.

30. Bouxsein, M. L.; Palermo, L.; Yeung, C.; Black, D. M., Digital x-ray radiogrammetry of the metacarpals predicts wrist, hip and vertebral fracture risk: A prospective analysis from the study of osteoporotic fractures. Bone 2001, 28, (5), S93-S93.

31. Courtney, A. C.; Wachtel, E. F.; Myers, E. R.; Hayes, W. C., Age-Related Reductions in the Strength of the Femur Tested in a Fall-Loading Configuration. Journal of Bone and Joint Surgery-American Volume 1995, 77A, (3), 387-395.

32. Brodsky, B.; Persikov, A. V., Molecular structure of the collagen triple helix. Fibrous Proteins: Coiled-Coils, Collagen and Elastomers 2005, 70, 301-+.

33. Conn, K. M.; Termine, J. D., Matrix Protein Profiles in Calf Bone-Development. Bone 1985, 6, (1), 33-36.

34. Zhu, W.; Robey, P. G.; Boskey, A. L., The regulatory role of matrix proteins in mineralization of bone. In Osteoporosis, R., M.; D., F.; D.A., N., Eds. Elsevier Academic Press: San Diego, CA, 2008; Vol. 1. 
35. Luo, G. B.; Ducy, P.; McKee, M. D.; Pinero, G. J.; Loyer, E.; Behringer, R. R.; Karsenty, G., Spontaneous calcification of arteries and cartilage in mice lacking matrix GLA protein. Nature 1997, 386, (6620), 78-81.

36. Boskey, A. L.; Maresca, M.; Ullrich, W.; Doty, S. B.; Butler, W. T.; Prince, C. W., Osteopontin-Hydroxyapatite Interactions in-Vitro - Inhibition of Hydroxyapatite Formation and Growth in a Gelatin-Gel. Bone and Mineral 1993, 22, (2), 147-159.

37. Sodek, J.; Chen, J. K.; Kasugai, S.; Nagata, T.; Zhang, Q.; Mckee, M. D.; Nanci, A., Elucidating the Functions of Bone Sialoprotein and Osteopontin in Bone-Formation. Chemistry and Biology of Mineralized Tissues 1992, 1002, 297-306 550.

38. Fujisawa, R.; Wada, Y.; Nodasaka, Y.; Kuboki, Y., Acidic amino acid-rich sequences as binding sites of osteonectin to hydroxyapatite crystals. Biochimica Et Biophysica Acta-Protein Structure and Molecular Enzymology 1996, 1292, (1), 53-60.

39. Morgan, E. F.; Barnes, G. L.; Einhorn, T. A., The bone organ system: form and funtion. In Osteoporosis, R., M.; D., F.; D.A., N., Eds. Elsevier Academic Press: San Diego, CA, 2008; Vol. 1.

40. Pozzo, D. C.; Hollabaugh, K. R.; Walker, L. M., Rheology and phase behavior of copolymer-templated nanocomposite materials. Journal of Rheology 2005, 49, (3), 759-782.

41. Palmer, L. C.; Newcomb, C. J.; Kaltz, S. R.; Spoerke, E. D.; Stupp, S. I., Biomimetic Systems for Hydroxyapatite Mineralization Inspired By Bone and Enamel. Chem. Rev. 2008, 108, (11), 4754-4783.

42. Enlow, D.; Rawal, A.; Kanapathipillai, M.; Schmidt-Rohr, K.; Mallapragada, S.; Lo, C. T.; Thiyagarajan, P.; Akinc, M., Synthesis and characterization of self-assembled block copolymer templated calcium phosphate nanocomposite gels. J. Mater. Chem. 2007, 17, (16), 1570-1578.

43. Kanapathipillai, M.; Yusufoglu, Y.; Rawal, A.; Hu, Y. Y.; Lo, C. T.; Thiyaigarajan, P.; Kalay, Y. E.; Akinc, M.; Mallapragada, S.; Schmidt-Rohr, K., Synthesis and characterization of ionic block copolymer templated calcium phosphate nanocomposites. Chem. Mater. 2008, 20, (18), 5922-5932. 
44. Yusufoglu, Y.; Hu, Y.; Kanapathipillai, M.; Kramer, M.; Kalay, Y. E.; Thiyagarajan, P.; Akinc, M.; Schmidt-Rohr, K.; Mallapragada, S., Bioinspired synthesis of selfassembled calcium phosphate nanocomposites using block copolymer-peptide conjugates. J. Mater. Res. 2008, 23, (12), 3196-3212.

45. Hu, Y. Y.; Yusufoglu, Y.; Kanapathipillai, M.; Yang, C. Y.; Wu, Y. Q.; Thiyagarajan, P.; Deming, T.; Akinc, M.; Schmidt-Rohr, K.; Mallapragada, S., Selfassembled calcium phosphate nanocomposites using block copolypeptide templates. Soft Matter 2009, 5, (21), 4311-4320.

46. Gan, Z. H., High-Resolution Chemical-Shift and Chemical-Shift Anisotropy Correlation in Solids Using Slow Magic Angle Spinning. J. Am. Chem. Soc. 1992, $114,(21), 8307-8309$.

47. Gan, Z. H.; Ernst, R. R., An improved 2D magic-angle-turning pulse sequence for the measurement of chemical-shift anisotropy. J. Magn. Reson., Ser. A 1996, 123, (1), 140-143.

48. Gullion, T., Measurement of Dipolar Interactions between Spin-1/2 and Quadrupolar Nuclei by Rotational-Echo, Adiabatic-Passage, Double-Resonance Nmr. Chemical Physics Letters 1995, 246, (3), 325-330.

49. Hing, A. W.; Vega, S.; Schaefer, J., Measurement of Heteronuclear Dipolar Coupling by Transferred-Echo Double-Resonance Nmr. Journal of Magnetic Resonance Series A 1993, 103, (2), 151-162.

50. Schmidt-Rohr, K.; Mao, J. D., Selective observation of nitrogen-bonded carbons in solid-state NMR by saturation-pulse induced dipolar exchange with recoupling. Chemical Physics Letters 2002, 359, (5-6), 403-411.

51. Schaefer, J.; Stejskal, E. O., C-13 Nuclear Magnetic-Resonance of Polymers Spinning at Magic Angle. Journal of the American Chemical Society 1976, 98, (4), 1031-1032.

52. Dixon, W. T., Spinning-Sideband-Free and Spinning-Sideband-Only Nmr-Spectra in Spinning Samples. J. Chem. Phys. 1982, 77, (4), 1800-1809.

53. Antzutkin, O. N.; Shekar, S. C.; Levitt, M. H., 2-Dimensional Side-Band Separation in Magic-Angle-Spinning Nmr. J. Magn. Reson., Ser. A 1995, 115, (1), 7-19. 
54. Gullion, T.; Schaefer, J., Rotational-Echo Double-Resonance Nmr. Journal of Magnetic Resonance 1989, 81, (1), 196-200.

55. Bak, M.; Sorensen, M. D.; Sorensen, E. S.; Rasmussen, L. K.; Sorensen, O. W.; Petersen, T. E.; Nielsen, N. C., The structure of the membrane-binding 38 C-terminal residues from bovine PP3 determined by liquid- and solid-state NMR spectroscopy. European Journal of Biochemistry 2000, 267, (1), 188-199.

56. Veshtort, M.; Griffin, R. G., SPINEVOLUTION: A powerful tool for the simulation of solid and liquid state NMR experiments. Journal of Magnetic Resonance 2006, 178, (2), 248-282.

57. Bertmer, M.; Eckert, H., Dephasing of spin echoes by multiple heteronuclear dipolar interactions in rotational echo double resonance NMR experiments. Solid State Nuclear Magnetic Resonance 1999, 15, (3), 139-152.

58. Gullion, T.; Pennington, C. H., theta-REDOR: an MAS NMR method to simplify multiple coupled heteronuclear spin systems. Chemical Physics Letters 1998, 290, (13), 88-93.

59. Jaroniec, C. P.; Tounge, B. A.; Rienstra, C. M.; Herzfeld, J.; Griffin, R. G., Measurement of C-13-N-15 distances in uniformly C-13 labeled biomolecules: Jdecoupled REDOR. Journal of the American Chemical Society 1999, 121, (43), 10237-10238.

60. deAzevedo, E. R.; Hu, W. G.; Bonagamba, T. J.; Schmidt-Rohr, K., Centerband-only detection of exchange: Efficient analysis of dynamics in solids by NMR. Journal of the American Chemical Society 1999, 121, (36), 8411-8412.

61. deAzevedo, E. R.; Bonagamba, T. J.; Schmidt-Rohr, K., Pure-exchange solid-state NMR. Journal of Magnetic Resonance 2000, 142, (1), 86-96.

62. deAzevedo, E. R.; Hu, W. G.; Bonagamba, T. J.; Schmidt-Rohr, K., Principles of centerband-only detection of exchange in solid-state nuclear magnetic resonance, and extension to four-time centerband-only detection of exchange. Journal of Chemical Physics 2000, 112, (20), 8988-9001. 
63. Reichert, D.; Bonagamba, T. J.; Schmidt-Rohr, K., Slow-down of C-13 spin diffusion in organic solids by fast MAS: A CODEX NMR study. Journal of Magnetic Resonance 2001, 151, (1), 129-135.

64. Schmidt-Rohr, K.; Spiess, H. W., Multidimensional Solid-State NMR and Polymers. 1994.

65. Luo, W.; Hong, M., Determination of the oligomeric number and intermolecular distances of membrane protein assemblies by anisotropic H-1-driven spin diffusion NMR spectroscopy. Journal of the American Chemical Society 2006, 128, (22), 72427251.

66. Schmidt-Rohr, K.; Clauss, J.; Spiess, H. W., Correlation of Structure, Mobility, and Morphological Information in Heterogeneous Polymer Materials by 2-Dimensional Wideline-Separation Nmr-Spectroscopy. Macromolecules 1992, 25, (12), 3273-3277.

67. Schmidt-Rohr, K.; Mao, J. D., Efficient CH-group selection and identification in C13 solid-state NMR by dipolar DEPT and H-1 chemical-shift filtering. Journal of the American Chemical Society 2002, 124, (46), 13938-13948.

68. Mao, J. D.; Schmidt-Rohr, K., Methylene spectral editing in solid-state C-13 NMR by three-spin coherence selection. Journal of Magnetic Resonance 2005, 176, (1), 1-6.

69. Munowitz, M. G.; Griffin, R. G., Two-Dimensional Nuclear Magnetic-Resonance in Rotating Solids - an Analysis of Line-Shapes in Chemical Shift-Dipolar Spectra. Journal of Chemical Physics 1982, 76, (6), 2848-2858.

70. Hong, M.; Gross, J. D.; Rienstra, C. M.; Griffin, R. G.; Kumashiro, K. K.; SchmidtRohr, K., Coupling amplification in 2D MAS NMR and its application to torsion angle determination in peptides. Journal of Magnetic Resonance 1997, 129, (1), 8592.

71. Mao, J. D.; Schmidt-Rohr, K., Separation of aromatic-carbon C-13 NMR signals from di-oxygenated alkyl bands by a chemical-shift-anisotropy filter. Solid State Nuclear Magnetic Resonance 2004, 26, (1), 36-45.

72. Herzfeld, J.; Berger, A. E., Sideband Intensities in Nmr-Spectra of Samples Spinning at the Magic Angle. Journal of Chemical Physics 1980, 73, (12), 6021-6030. 
73. Liu, S. F.; Mao, J. D.; Schmidt-Rohr, K., A robust technique for two-dimensional separation of undistorted chemical-shift anisotropy powder patterns in magic-anglespinning NMR. Journal of Magnetic Resonance 2002, 155, (1), 15-28.

74. Hou, S. S.; Beyer, F. L.; Schmidt-Rohr, K., High-sensitivity multinuclear NMR spectroscopy of a smectite clay and of clay-intercalated polymer. Solid State Nuclear Magnetic Resonance 2002, 22, (2-3), 110-127. 


\title{
Chapter 2
}

\section{Strongly bound citrate stabilizes the apatite nanocrystals in bone}

A paper published in the Proceedings of the National Academy of Science 2010, 107, 22425-22429

\author{
Y.-Y. Hu, A. Rawal, K. Schmidt-Rohr
}

Ames Laboratory and Department of Chemistry, Iowa State University, Ames, IA 50011, USA

\begin{abstract}
Nanocrystals of apatitic calcium phosphate impart the organic-inorganic nanocomposite in bone with favorable mechanical properties. So far, the factors preventing crystal growth beyond the favorable thickness of ca. $3 \mathrm{~nm}$ have not been identified. Here we show that the apatite surfaces are studded with strongly bound citrate molecules, whose signals have been identified unambiguously by multinuclear magnetic resonance (NMR) analysis. NMR reveals that bound citrate accounts for $5.5 \mathrm{wt} \%$ of the organic matter in bone and covers apatite at a density of about 1 molecule per $(2 \mathrm{~nm})^{2}$, with its three carboxylate groups at distances of 0.3 to $0.45 \mathrm{~nm}$ from the apatite surface. Bound citrate is highly conserved, being found in fish, avian, and mammalian bone, which indicates its critical role in interfering with crystal thickening and stabilizing the apatite nanocrystals in bone.
\end{abstract}

\section{Introduction}

The load-bearing material in bone is a fascinating organic-inorganic nanocomposite whose stiffness is provided by thin nanocrystals of carbonated apatite, a calcium phosphate, imbedded in an organic matrix consisting mostly of collagen, a fibrous protein (1-5). The small (ca. 3-nm) thickness of the apatite nanocrystals is favorable for mechanical properties, likely preventing crack propagation (6). While the size and shape of the nanocrystals have been studied extensively $(4,5)$, the mechanism stabilizing them at a thickness corresponding to only about 4 unit cells has not been elucidated. A better understanding of the factors controlling the nanocrystals in bone is desirable for prevention and treatment of bone diseases such as osteoporosis, which causes millions of fractures each year (7), and for more 
efficient synthesis of biomimetic nanocomposites $(8,9)$. In-vitro experiments have shown that carboxylate-rich proteins such as osteocalcin and osteopontin (7) can affect hydroxyapatite crystal formation and growth $(10,11)$. These observations might suggest that such proteins limit nanocrystal thickening (12); however, these proteins are not sufficiently abundant in vivo to bind to all the nanocrystal surfaces at high enough area concentration; possibly, they control the length of the nanocrystals (7).

Here we show instead that the surfaces of the apatite crystals in bone are studded with strongly bound citrate molecules, at a density of ca. $1 /(2 \mathrm{~nm})^{2}$, using advanced solid-state NMR as a unique tool for probing buried interfaces. Citrate is quite abundant in bone (ca. 1 $\mathrm{wt} \%$, or $5 \mathrm{wt} \%$ of the organic components) $(13,14)$. Before 1975 , citrate in bone was studied by simple wet-chemical methods and thought to regulate bone demineralization (14). However, citrate is no longer even mentioned in most of the prominent literature on the bone nanocomposite published during the last thirty years $(1-5,15-18)$. We now highlight the importance of citrate in bone by demonstrating that it is not a dissolved calcium-solubilizing agent but a strongly bound, integral part of the nanocomposite. Structurally, citrate stands out among biomolecules in that it contains an oxygen-bonded quaternary (nonprotonated) alkyl carbon, which we have identified by NMR spectral editing. We show that citrate signals dominate the NMR spectra of carbons near the interface with apatite and estimate distances from the apatite surface. Further, we determine the area concentration of apatite-bound citrate and confirm it by quantitative analysis of spin exchange between ${ }^{13} \mathrm{C}$-labeled citrate adsorbed to purified bone apatite. Thus, a quite comprehensive picture emerges of citrate binding strongly to apatite and inhibiting an increase in apatite nanocrystal thickness.

\section{Results and Discussion}

Fig. 2.1A-C shows the ${ }^{13} \mathrm{C}$ NMR spectra of fish, avian, and bovine bone. Similar to previously published (17-19), the spectra are dominated by signals of collagen, the fibrous protein rich in glycine (33\%), proline, hydroxyproline, and alanine (each 11\%) that forms the matrix of the bone nanocomposite. The spectrum of ${ }^{13} \mathrm{C}$ near ${ }^{31} \mathrm{P}$ in bovine bone, obtained by ${ }^{13} \mathrm{C}\left\{{ }^{31} \mathrm{P}\right\}$ rotational echo double resonance (REDOR) $\operatorname{NMR}(17,20,21)$, i.e. with ${ }^{13} \mathrm{C}$ observation and ${ }^{31} \mathrm{P}$ recoupling pulses, is shown in Fig. 2.1D. Strong signals are observed at 
$182,169,76$, and $49 \mathrm{ppm}$; those at 182 and $76 \mathrm{ppm}$, as well as the inorganic carbonate signal at $169 \mathrm{ppm}$, had been detected before $(17,18)$, but the strong signal intensity near $49 \mathrm{ppm}$ had not been recognized. We assign the peaks at 182, 76, and $49 \mathrm{ppm}$ to citrate (see structure at top of Fig. 2.1) bound to the apatite surface, since the strong citrate peaks of bone partially exchanged with uniformly ${ }^{13} \mathrm{C}$-labeled citrate (Fig. $2.1 \mathrm{E}$ ) and the signal positions of calcium citrate (Fig. $2.1 F$ ) provide an excellent match. Citrate contains an oxygen-bonded quaternary (nonprotonated) alkyl carbon, which is quite rare among biomolecules; the slow H-C dipolar dephasing of the 76-ppm signal in both citrate and bone (Fig. 2.1G and 2.1H) is a telltale sign of this nonprotonated carbon and thus of citrate. The weak C-H dipolar coupling cannot be attributed to mobility effects, since the ${ }^{1} \mathrm{H}$ wideline spectrum (22) associated with the 76-ppm carbon shows no motional narrowing (Fig. 2.2). We agree with ref. (17) that the 76- and 182-ppm signals are not from collagen, but the subsequent assignment to sugar residues (18, 19 ) is not tenable for the majority of this signal, since the $\mathrm{C}-\mathrm{OH}$ carbons in sugars are protonated, not quaternary. The dominant contribution of the nonprotonated carbon of citrate at $76 \mathrm{ppm}$ and the weak signal of anomeric (O-C-O) carbons near $100 \mathrm{ppm}$, which is a band characteristic of sugar rings (23), indicates that the polysaccharide concentration is only about $1 / 4$ of that of citrate. The intensity of the 76-ppm quaternary citrate carbon peak ( $0.75 \%$ of the total spectrum) corresponds to a $4.5 \%$ signal fraction of all six citrate carbons and ca. $5.5 \mathrm{wt} \%$ of citrate in the organic component of bone, consistent with wet-chemical analyses in the literature $(13,14)$.

In order to provide a more detailed picture of the bound citrate, we have determined the distances of various citrate carbons from the apatite surface by analysis of the 76- and 182-ppm signals in bone and in bone exchanged with uniformly ${ }^{13} \mathrm{C}$-labeled citrate, using ${ }^{13} \mathrm{C}\left\{{ }^{31} \mathrm{P}\right\}$ REDOR NMR (filled symbols in Fig. 2.3A,B). A smaller distance gives rise to stronger ${ }^{13} \mathrm{C}^{31} \mathrm{P}$ dipolar couplings and a faster REDOR signal decay. The almost complete long-time dephasing in ${ }^{13} \mathrm{C}\left\{{ }^{31} \mathrm{P}\right\}$ REDOR NMR (Fig. 2.3A) proves that most water-insoluble citrate is close to ${ }^{31} \mathrm{P}$, i.e. bound to apatite rather than precipitated as sodium or calcium citrate. Multispin analysis of these REDOR data yields a distance of about $0.35 \mathrm{~nm}$ between the top ${ }^{31} \mathrm{P}$ layer and citrate carboxylates (for details see Supporting Information). For citrate with ${ }^{13} \mathrm{C}$-labeled terminal $\mathrm{COO}^{-}$groups, peaks at 178 and $180 \mathrm{ppm}$ can be deconvoluted. The 
dephasing of the two peaks is somewhat different, see Fig. 2.3A, corresponding to 0.33 and $0.45( \pm 0.05) \mathrm{nm}$ from the top ${ }^{31} \mathrm{P}$ layer, respectively. Strongly asymmetric binding of only one terminal carboxylate to the apatite surface, similar as in simulations of citric acid on hydroxyapatite (24), would result in distances of 0.3 and $0.7 \mathrm{~nm}$; most citrate molecules do not "stand up" like this on bone apatite. Figure $3 B$ shows the REDOR dephasing of other carbons in citrate. On average, the carboxylate carbons are slightly closer to the interface than methylene and quaternary $\mathrm{C}$, consistent with carboxylate binding to calcium of apatite. All citrate carbons are found at about $0.4 \mathrm{~nm}$ from the top phosphorus layer, which means that the long axes of the citrate molecules are titled slightly relatively to the apatite surface. The ${ }^{13} \mathrm{C}\left\{{ }^{31} \mathrm{P}\right\}$ REDOR data also exclude that phosphocitrate, which has been proposed as a major form of citrate in bone (14), is significantly present; the corresponding simulated curve (dashed) for a two-bond ${ }^{13} \mathrm{C}-{ }^{31} \mathrm{P}$ distance shows a much faster decay than is observed experimentally.

The citrate area concentration on bone apatite can be calculated from the 3-nm thickness of the crystallites, the composite density of $2.2 \mathrm{~g} / \mathrm{cm}^{3}, 50 \mathrm{vol} \%$ apatite, and $1 \mathrm{wt} \%$ citrate (see Supporting Information). The resulting value of about 1 molecule per $(2 \mathrm{~nm})^{2}$ agrees with that found for citrate on purified bone mineral (deproteinized bovine bone, NuOss ${ }^{\mathrm{TM}}$ ) from the citrate concentration determined by ${ }^{13} \mathrm{C}$ NMR $(0.8 \mathrm{mg}$ citrate per $190 \mathrm{mg}$ of bone mineral) and the specific surface area of $60 \mathrm{~m}^{2} / \mathrm{g}$. Given that a citrate molecule has a geometrical area of ca. $0.65 \mathrm{~nm}^{2}$ (25), citrate covers about $1 / 6$ of the available apatite surface area in bone.

In order to investigate the distribution of citrate in more detail, we have probed the local citrate density in terms of ${ }^{13} \mathrm{C}^{13} \mathrm{C}$ dipolar couplings of ${ }^{13} \mathrm{C}$-labeled terminal $\mathrm{COO}^{-}$ groups in citrate bound to the purified $\mathrm{NuOss}^{\mathrm{TM}}$ bone mineral, using ${ }^{13} \mathrm{C}$ centerband-only detection of exchange (CODEX) NMR with dipolar ${ }^{13} \mathrm{C}$ spin exchange (26). In short, CODEX NMR yields the fraction of ${ }^{13} \mathrm{C}$ spins that, during a waiting period $\mathrm{t}_{\mathrm{m}}$, have not changed their frequency due to dipolar spin exchange with other ${ }^{13} \mathrm{C}$ nuclear magnets (26). If the doubly ${ }^{13} \mathrm{C}$-labeled citrate molecules were isolated from their neighbors, the CODEX data would level off at a long-time value of 1/2 (ref. (26)). The experimental data in Fig. 2.4 drop below $1 / 2$, proving contacts between different citrate molecules on a $1.5-\mathrm{nm}$ scale. The fast 
initial decay is due to the ${ }^{13} \mathrm{C}$ spins separated by $0.5 \mathrm{~nm}$ within each molecule, while the slope at longer times reflects mostly the distance to the nearest-neighbor molecule. Simulations (described in the Supporting Information) give good fits for area densities near the value of $1 /(2 \mathrm{~nm})^{2}$ calculated above, with moderate positional ordering of citrate molecules. This result shows that the molecules cover most apatite surfaces with the average area density.

Proof of citrate immobilization can be obtained from the NMR data. The CODEX signal decay would be much more pronounced than observed in Fig. 2.4 if large-amplitude motions of the carboxylate groups occurred on the 10-s time scale (26). Large-amplitude motions on the 10- $\mu$ s (or faster) timescale are excluded by the absence of motional narrowing in ${ }^{1} \mathrm{H}^{13} \mathrm{C}$ wide-line separation NMR, see Fig. 2.2, confirming strong binding of citrate to bone apatite. Thus, citrate cannot be part of a surface layer of hydrated mobile ions proposed by Rey et al. (3).

Based on these structural data, we propose the model of citrate bound to apatite presented in Fig. 2.5, with a realistic citrate density and orientation. The $\mathrm{CH}_{2}$ groups of citrate facing the exterior may give the surface a locally less hydrophilic character, making it more compatible with the nonpolar proline and alanine residues of the collagen matrix. Apatite is represented as ideal hydroxyapatite, $\mathrm{Ca}_{10}\left(\mathrm{PO}_{4}\right)_{6}(\mathrm{OH})_{2}$ (hexagonal unit cell with a $=\mathrm{b}=0.944 \mathrm{~nm}, \mathrm{c}=0.688 \mathrm{~nm}$ ). Calcium ions in two planes of highest morphological importance (27) are shown on the top left and right. In the $(10 \overline{1} 0)_{1}$ plane, $\mathrm{Ca}^{2+}$ is spaced by $\mathrm{c} / 2=0.34 \mathrm{~nm}$, which matches the $0.32 \mathrm{~nm}$ spacing between the centers of the three $\mathrm{COO}^{-}$ groups of citrate. In the $(10 \overline{1} 0)_{2}$ plane, spacings of $c=0.688 \mathrm{~nm}$ match the distance between the terminal carboxylates of citrate. The structure shown is an idealization of the calciumand hydroxide-deficient carbonated bone apatite; the actual surface is probably reconstructed (28) and significantly disordered. Adsorption of citrate purely at defects, such as steps on certain crystal faces, seems unlikely given its relatively high area density, and would result in stronger clustering of citrate, at the defects, than observed in CODEX NMR.

Citrate in bone is fundamentally different from carbonate, fluoride, sodium, magnesium, hydroxide (29), calcium, or phosphate ions, in that it is too large to be incorporated into the apatite crystal lattice. Therefore, bound citrate must remain an 
interfacial component that affects properties of the bone nanocomposite more profoundly than do simple substitutions of carbonate for phosphate or of sodium for calcium. Thus, we propose that citrate bound to the nanocrystal surface stabilizes the apatite nanocrystals by preventing their further growth. The crystallite thickness of $3 \mathrm{~nm}$ corresponds to only 4 unit cells, which results in a high surface energy that would make further crystal thickening favorable. Strongly bound citrate interferes with that thickening by inhibiting formation of additional phosphate layers. Indeed, it has been shown in vitro that hydroxyapatite forms thinner nanocrystals in the presence of citrate (30-32). In vivo, addition of citrate to calcium phosphate biocement for bone implants improves biocompatibility $(32,33)$, and various citrate nutritional supplements appear to prevent the onset of osteoporosis (34), presumably by stabilizing the apatite crystals.

Citrate also stabilizes hydroxyapatite relative to other calcium phosphates (35), possibly due to the good match between the spacing of the terminal $\mathrm{COO}^{-}$groups in citrate and the lattice parameter $c$ in apatite. Furthermore, citrate bound to collagen, unlike neat collagen (7), initiates apatite crystallization in vitro (36). Thus, citrate might play an important role not only in stabilizing existing apatite nanocrystals, but also in crystal nucleation during biomineralization in bone. The importance of citrate in bone, which accounts for $\sim 80 \%$ of all citrate in the body (14), is confirmed by its presence in a wide range of vertebrate classes, from fish to mammals, see Fig. 2.1A-C. Citrate provides a larger number of carboxylates for calcium binding in bone than do the proteins osteocalcin (37), osteopontin, and osteonectin (7) taken together.

\section{Conclusions and Outlook}

By means of advanced solid-state NMR spectroscopy and distance measurements, we have demonstrated that citrate is strongly bound to the apatite nanocrystals in bone. Citrate accounts for $5.5 \mathrm{wt} \%$ of the organic fraction of the nanocomposite in bone, and thus provides more $\mathrm{COO}^{-}$groups for binding to calcium of apatite than all non-collagenous proteins in bone combined. The strong binding of citrate was proved by its close distance to apatite and absence of large-amplitude motions. After removing native citrate in bone by hot dilute acid treatment and replacing it with ${ }^{13} \mathrm{C}$-labeled citrate, the distances of all carbons in citrate from 
the apatite surface were measured. Two of the three carboxylate carbons are slightly closer to the surface than are methylene and quaternary $\mathrm{C}$, whose similar distances of $\sim 0.4 \mathrm{~nm}$ show that the long axis of most citrate molecules is tilted only slightly relative to the surface. The spacing of $\mathrm{COO}^{-}$groups in citrate matches that of calcium ions along the $c$-axis in apatite. The area density of citrate on bone apatite is around $1 /(2 \mathrm{~nm})^{2}$, sufficient to make the nanocrystal surface less hydrophilic. On the basis of these data, a model of citrate bound to apatite was presented. Bound apatite clearly interferes with nanocrystal thickening and is found similarly in a wide range of vertebrate orders, which indicates its important role in stabilizing the size of the nanocrystals at the small thickness most favorable for mechanical properties and for fast resorption during bone remodeling.

The discovery of apatite-bound citrate in the bone nanocomposite leads to various intriguing research questions. What is the source of citrate in bone? At what stage of bone development does citrate appear? How is the abundance of citrate on the nanocrystal surface controlled? Is the citrate concentration abnormal in diseased bone? Further, the measured NMR distance data will be valuable as benchmarks for ab-initio simulations of surface binding of functional molecules.

\section{Methods}

Compact (cortical) bone was obtained from the midsections of femur bone from a four-year-old cow and three-month-old chicken and cleaned of all muscular and connective tissue, and of the marrow. The poorly mineralized and porous surface layer of the femur was cut away with a sharp razor. Fish bone was obtained from the ribs of tilapia, a fresh-water fish, which was cooled to below $4^{\circ} \mathrm{C}$ shortly after the animal had been sacrificed. The bone was cryo-milled in a SPEX 6750 Freezer Mill cryogenic grinder, washed in a 3:1 mixture of methanol and chloroform to remove any lipids, placed under vacuum at 0.01 Torr and $233 \mathrm{~K}$ to remove all traces of solvent, equilibrated at ambient humidity, and packed into 7-mm and 4-mm zirconia NMR rotors with Kel-F® caps.

${ }^{13} \mathrm{C}$-labeled citrate exchanged bovine bone was prepared with $1,5-{ }^{13} \mathrm{C}_{2}, 2,4-{ }^{13} \mathrm{C}_{2}$, or ${ }^{13} \mathrm{C}_{6}$ citric acid, all obtained from Isotec. We made $2 \mathrm{mM}$ citrate solutions by dissolving 5 $\mathrm{mg}$ of each ${ }^{13} \mathrm{C}$-labeled citric acid in $13 \mathrm{~mL}$ deionized water, and adjusted the $\mathrm{pH}$ to 7.4 by 
using $\mathrm{NaOH}$. In order to extract native citrate, degreased bovine bone powder was boiled at $80^{\circ} \mathrm{C}$ for 24 hours in dilute acid made from distilled water adjusted to $\mathrm{pH}=4$ using hydrochloric acid. The bone powder was removed from the dilute acid and washed until the supernatant was $\mathrm{pH}$ neutral, then incubated in the $2 \mathrm{mM}$ citrate solution at $36.5^{\circ} \mathrm{C}$ for 7 days under gentle shaking, in order to allow the ${ }^{13} \mathrm{C}$-labeled citrate to diffuse into the bone. Next, the citrate-exchanged bovine bone was washed several times with deionized water to remove loosely attached citrate. The residual water was removed via lyophilization.

The ${ }^{13} \mathrm{C}$-labeled citrate adsorbed onto the surface of $\mathrm{NuOss}^{\mathrm{TM}}$ bone mineral (deproteinized bovine bone, ACE Surgical Supply Co., Brockton, MA) with particle size of 0.5-1.0 mm and specific surface area of $60 \mathrm{~m}^{2} / \mathrm{g}$ was prepared as follows. We prepared 13 $\mathrm{mL}$ of $2 \mathrm{mM}$ citrate solution by dissolving $5 \mathrm{mg}$ citric-1,5- ${ }^{13} \mathrm{C}_{2}$ acid in $13 \mathrm{~mL}$ deionized water, and adjusted the $\mathrm{pH}$ to 7.4 using $\mathrm{NaOH}$. After adding the bone mineral matrix into the citrate solution, the mixture was incubated at $36.5^{\circ} \mathrm{C}$ with continuous gentle shaking overnight. The supernatant was decanted and the bone mineral was washed several times to remove loosely attached citrate. Residual water was removed by freeze-drying.

The NMR experiments were performed using a Bruker DSX-400 spectrometer at 100 $\mathrm{MHz}$ for ${ }^{13} \mathrm{C}$, and $162 \mathrm{MHz}$ for ${ }^{31} \mathrm{P}$, in Bruker 7-mm double resonance (for spectroscopy and CODEX) and 4-mm triple resonance (for REDOR) magic-angle spinning probes at spinning frequencies between 4 and $6 \mathrm{kHz}$. The $90^{\circ}$ pulse lengths were ca. $4.2 \mu \mathrm{s}$. Most of the ${ }^{13} \mathrm{C}$ spectra in Fig. 2.1 were recorded under magic angle spinning of $5.3 \mathrm{kHz}$ with total suppression of sidebands (TOSS) (38). The cross polarization contact time was $1 \mathrm{~ms}$ and the recycle delay $2.5 \mathrm{~s}$. To select signals of carbons with weak C-H dipolar couplings, spectra were also acquired with additional $40 \mu \mathrm{s}$ of gated decoupling before signal detection. ${ }^{1} \mathrm{H}-{ }^{13} \mathrm{C}$ wideline separation spectra were measured on bovine bone at $4.3 \mathrm{kHz}$ magic-angle spinning (MAS), with $0.2 \mathrm{~ms}$ standard Hartmann-Hahn cross polarization and $60 \mathrm{t}_{1}$-increments of 10 $\mu$ s, using multi-echo detection (39) with spectral resolution (40) for sensitivity enhancement and reduction of the measuring time by a factor of about 10 .

${ }^{13} \mathrm{C}\left\{{ }^{31} \mathrm{P}\right\}$ REDOR (20) NMR data were obtained using a 4-mm Bruker triple resonance probehead at $6 \mathrm{kHz}$ MAS with recoupling times of $\mathrm{Nt}_{\mathrm{r}}=3 \mathrm{~ms}, 4.66 \mathrm{~ms}, 6 \mathrm{~ms}$, and $7.66 \mathrm{~ms}$. A four-step phase cycle along with composite $90^{\circ}-180^{\circ}-90^{\circ}{ }^{31} \mathrm{P}$ recoupling pulses 
was used to minimize pulse length errors and achieve maximal dephasing (41). The ${ }^{1} \mathrm{H}$ composite-pulse decoupling power was $\gamma \mathrm{B}_{1} /(2 \pi)=66 \mathrm{kHz}$. Figure $1 D$ shows the sum of the ${ }^{13} \mathrm{C}\left\{{ }^{31} \mathrm{P}\right\}$ REDOR difference spectra $\Delta \mathrm{S}=\mathrm{S}_{0}-\mathrm{S}$, where $\mathrm{S}_{0}$ is the reference signal without pulses applied to ${ }^{31} \mathrm{P}$. The total measuring time for the REDOR spectra of bone was 19 days. In uniformly ${ }^{13} \mathrm{C}$ labeled citrate, ${ }^{13} \mathrm{C}-{ }^{13} \mathrm{C}$ J-couplings result in fast loss of the reference signal $\mathrm{S}_{0}$ when the standard REDOR sequence is used. Therefore, the dephasing of $\mathrm{C}_{\mathrm{q}}$ in uniformly ${ }^{13} \mathrm{C}$-labeled citrate was measured by applying a selective soft Gaussian pulse of $\gamma \mathrm{B}_{1} /(2 \pi)=1$ $\mathrm{kHz}$, which removes the J-coupling effects (42), to the $\mathrm{C}_{\mathrm{q}}$ resonance. CODEX ${ }^{13} \mathrm{C}$ NMR data were obtained for $1,5-{ }^{13} \mathrm{C}_{2}$ citrate adsorbed onto the surface of NuOss ${ }^{\mathrm{TM}}$ bone mineral at 4$\mathrm{kHz}$ MAS with mixing times up to $20 \mathrm{~s}$, and multi-echo detection was used for sensitivity enhancement.

Acknowledgments. This work was supported by the U.S. Department of Energy, Office of Basic Energy Science, Division of Materials Sciences \& Engineering, at the Ames Laboratory, under Contract No. DE-AC02-07CH11358.

\section{References}

1. Buckwalter JA, Glimcher MJ, Cooper RR, \& Recker R (1995) Bone Biology .1. Structure, Blood-Supply, Cells, Matrix, and Mineralization. J. Bone. Joint. Surg. Am. 77A, 1256-1275.

2. Currey JD (2002) Bones (Princeton University Press, Princeton, New Jersey) p 456.

3. Rey C, Combes C, Drouet C, \& Glimcher MJ (2009) Bone mineral: update on chemical composition and structure (vol 20, pg 1013, 2009). Osteoporos. Int. 20, 2155-2155.

4. Weiner S \& Traub W (1992) Bone-Structure - from Angstroms to Microns. FASEB J. 6, 879-885.

5. Weiner S \& Wagner HD (1998) The material bone: Structure mechanical function relations. Annu. Rev. Mater. Sci. 28, 271-298. 
6. Gao HJ, Ji BH, Jager IL, Arzt E, \& Fratzl P (2003) Materials become insensitive to flaws at nanoscale: Lessons from nature. Proc. Natl. Acad. Sci. U.S.A. 100, 55975600 .

7. Zhu W, Robey PG, \& Boskey AL (2008) The Regulatory Role of Matrix Proteins in Mineralization of Bone. Osteoporosis, eds Markus R, Feldman D, Nelson DA, \& Rosen CJ (Elsevier Academic Press, San Diego, CA), Vol 1.

8. Hartgerink JD, Beniash E, \& Stupp SI (2001) Self-assembly and mineralization of peptide-amphiphile nanofibers. Science 294, 1684-1688.

9. $\mathrm{Hu}$ YY, et al. (2009) Self-assembled calcium phosphate nanocomposites using block copolypeptide templates. Soft Matter 5, 4311-4320.

10. Johnsson M, Richardson CF, Bergey EJ, Levine MJ, \& Nancollas GH (1991) The Effects of Human Salivary Cystatins and Statherin on Hydroxyapatite Crystallization. Archs. Oral Biol. 36, 631-636.

11. Hunter GK, Kyle LC, \& Goldberg HA (1994) Modulation of crystal formation by bone phosphoproteins: structural specificity of the osteopontin-mediated inhibition of hydroxyapatite formation. Biochem. J. 300, 723-728.

12. Spoerke ED, Anthony SG, \& Stupp SI (2009) Enzyme Directed Templating of Artifical Bone Mineral. Adv. Mater. 21, 425-430.

13. Dickens F (1941) The citric acid content of animal tissues, with reference to its occurrence in bone and tumor. Biochem. J. 35, 1011-1023.

14. Hartles RL (1964) Citrate in Mineralized Tissues Adv. Oral Biol. 1, 225-253.

15. Currey JD (2003) Role of collagen and other organics in the mechanical properties of bone. Osteoporos. Int. 14, S29-S36.

16. Posner AS \& Beebe RA (1975) Surface chemistry of bone mineral and related calcium phosphates. Semin. Arthritis. Rheum. 4, 267-291.

17. Jaeger C, et al. (2005) Investigation of the Nature of the Protein-Mineral Interface in Bone by Solid-State NMR. Chem. Mat. 17, 3059-3061.

18. Wise ER, et al. (2007) The organic-mineral interface in bone is predominantly polysaccharide. Chem. Mater. 19, 5055-5057. 
19. Zhu PZ, et al. (2009) Time-Resolved Dehydration-induced Structural Changes in an Intact Bovine Cortical Bone Revealed by Solid-State NMR Spectroscopy. J. Am. Chem. Soc. 131, 17064-17065.

20. Gullion T \& Schaefer J (1989) Detection of weak heteronuclear couplings by REDOR. Adv. Magn. Reson. 13, 57-83.

21. Ndao M, et al. (2009) A ${ }^{13} \mathrm{C}\left({ }^{31} \mathrm{P}\right)$ REDOR NMR Investigation of the Role of Glutamic Acid Residues in Statherin-Hydroxyapatite Recognition. Langmuir 25, 12136-12143.

22. Schmidt-Rohr K, Clauss J, \& Spiess HW (1992) Correlation of Structure, Mobility, and Morphological Information in Heterogeneous Polymer Materials by TwoDimensional Wide-line Separation NMR Spectroscopy. Macromolecules 25, 32733277.

23. Best SM, Duer MJ, Reid DG, Wise ER, \& Zou S (2008) Towards a model of the mineral-organic interface in bone: NMR of the structure of synthetic glycosaminoglycan- and polyaspartate-calcium phosphate composites. Mag. Reson. Chem. 46, 323-329.

24. de Leeuw NH \& Rabone JAL (2007) Molecular dynamics simulations of the interaction of citric acid with the hydroxyapatite (0001) and (01 $\overline{1} 0)$ surfaces in an aqueous environment. Crystengcomm 9, 1178-1186.

25. Misra DN (1996) Interaction of Citric Acid with Hydroxyapatite: Surface Exchange of Ions and Precipitation of Calcium Citrate. J. Dent. Res. 75, 1418-1425.

26. deAzevedo ER, Hu W-G, Bonagamba TJ, \& Schmidt-Rohr K (1999) CenterbandOnly Detection of Exchange: Efficient Analysis of Dynamics in Solids by NMR. $J$. Am. Chem. Soc. 121, 8411-8412.

27. Terpstra RA, et al. (1986) F-Faces of Apatite and Its Morphology - Theory and Observation. J. Cryst. Growth 78, 468-478.

28. Mathew M \& Takagi S (2001) Structures of biological minerals in dental research. $J$. Res. Natl. Inst. Stand. Technol. 106, 1035-1044.

29. Cho G, Wu Y, \& Ackerman JL (2003) Detection of Hydroxyl Ions in Bone Mineral by Solid-State NMR Spectroscopy. Science 300, 1123-1127. 
30. Tenhuisen KS \& Brown PW (1994) The effects of citric and acetic acids on the formation of calcium-deficient hydroxyapatite at $38^{\circ}$ C. J. Mater. Sci.: Mater. Med. 5, 291-298.

31. Lopez-Macipe A, Gomez-Morales J, \& Rodriguez-Clemente R (1998) Nanosized Hydroxyapatite Precipitation from Homogeneous Calcium/Citrate/Phosphate Solutions Using Microwave and Conventional Heating. Adv. Mater. 10, 49-53.

32. Hempel U, Reinstorf A, Poppe W, \& Wenzel KW (2004) Proliferation and differentiation of osteoblasts on Biocement D modified with collagen type I and citric acid. J. Biomed. Mater. Res. B Appl. Biomater. 71, 130-143.

33. Schneiders W, et al. (2007) Effect of modification of hydroxyapatite/collagen composites with sodium citrate, phosphoserine, phosphoserine/RGD-peptide and calcium carbonate on bone remodelling. Bone 40, 1048-1059.

34. Karp HJ, Ketola ME, \& Lamberg-Allardt CJE (2009) Acute effects of calcium carbonate, calcium citrate, and potassium citrate on markers of calcium and bone metabolism in young women. Br. J. Nutr. 102, 1341-1347.

35. Jiang WG, et al. (2009) Biomimetically Triggered Inorganic Crystal Transformation by Biomolecules: A New Understanding of Biomineralization. J. Phys. Chem. B 113, 10838-10844.

36. Rhee SH \& Tanaka J (1999) Effect of citric acid on the nucleation of hydroxyapatite in a simulated body fluid. Biomaterials 20, 2155-2160.

37. Hoang QQ, Sicheri F, Howard AJ, \& Yang DSC (2003) Bone recognition mechanism of porcine osteocalcin from crystal structure. Nature 425, 977-980.

38. Dixon WT (1982) Spinning-sideband-free and spinning-sideband-only NMR spectra of spinning samples. J. Chem. Phys. 77, 1800-1809.

39. Hou SS, Beyer FL, \& Schmidt-Rohr K (2002) High-Sensitivity Multinuclear NMR Spectroscopy of a Smectite Clay and of Clay-Intercalated Polymer. Solid State NMR 22, 110-127.

40. Trebosc J, Wiench JW, Huh S, Lin VSY, \& Pruski M (2005) Studies of organically functionalized mesoporous silicas using heteronuclear solid state correllation NMR spectroscopy under fast magic-angle-spinning. J. Am. Chem. Soc. 127, 7587-7593. 
41. Sinha N, Schmidt-Rohr K, \& Hong M (2004) Compensation of pulse imperfections in rotational-echo double resonance NMR by composite pulses and EXORCYCLE. $J$. Magn. Reson. 168, 358-365.

42. Jaroniec CP, Tounge BA, Rienstra CM, Herzfeld J, \& Griffin RG (1999) Measurement of ${ }^{13} \mathrm{C}-{ }^{15} \mathrm{~N}$ distances in uniformly ${ }^{13} \mathrm{C}$ labeled biomolecules: $\mathrm{J}$ decoupled REDOR. J. Am. Chem. Soc. 121, 10237-10238.

\section{Figures}

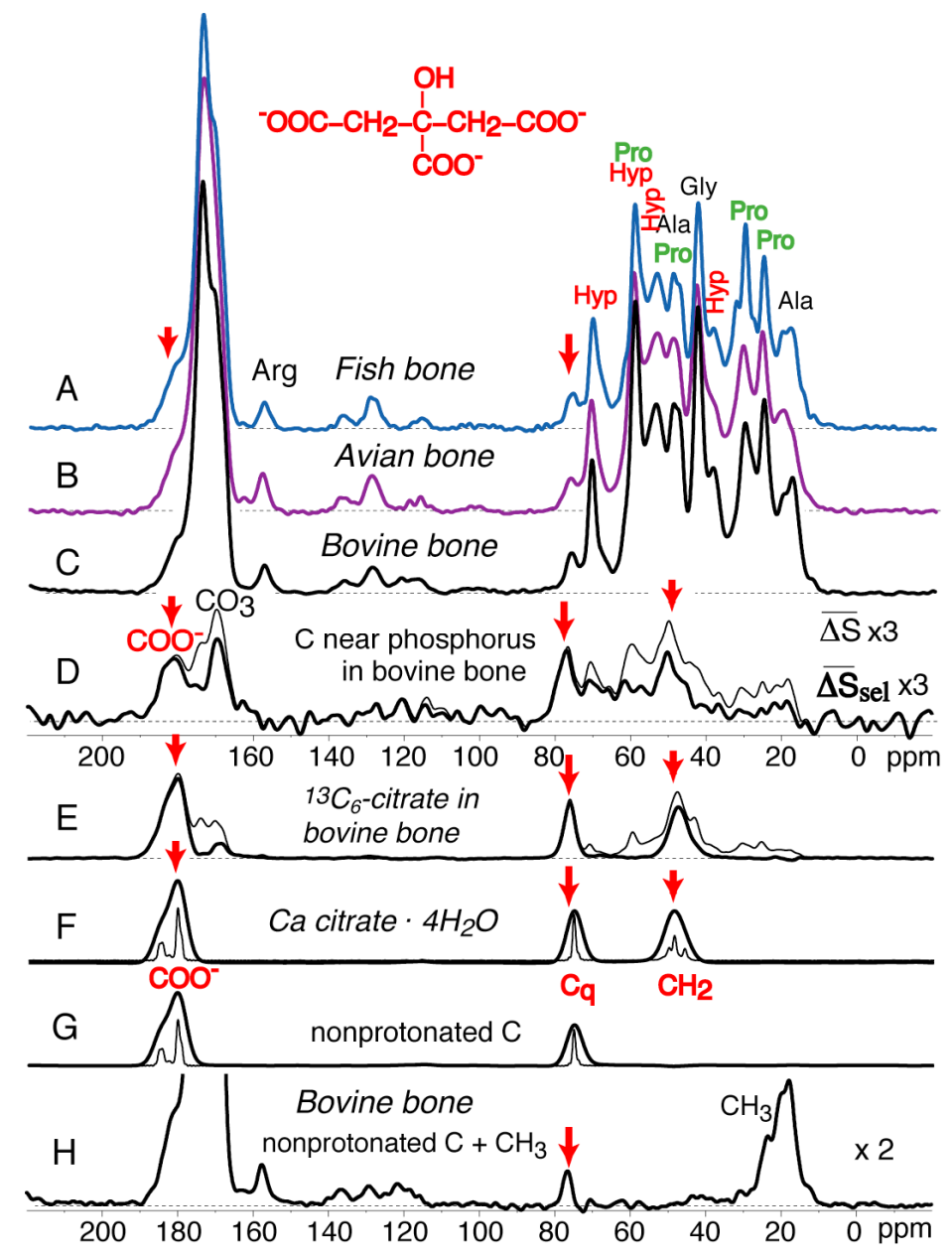

Fig. 2.1. ${ }^{13} \mathrm{C}$ NMR spectra of bone, of organic residues at the interface with apatite, and of ${ }^{13} \mathrm{C}$-labeled citrate in bone. (A) Fish bone; (B) avian bone; (C) bovine bone. (D) Spectra of ${ }^{13} \mathrm{C}$ near ${ }^{31} \mathrm{P}$ in bovine bone. Thin line: ${ }^{13} \mathrm{C}\left\{{ }^{31} \mathrm{P}\right\}$ REDOR difference $(\Delta \mathrm{S})$ spectrum (total 
measuring time: 19 days). Thick line: Same data but with the spectrum S scaled up by 1.1 to match the 43-ppm peak of Gly in $\mathrm{S}_{0}$ and thus remove signal of abundant interior collagen residues. (E) Thin line: Spectrum of bovine bone with uniformly ${ }^{13} \mathrm{C}$-labeled bound citrate, introduced after (partial) removal of native citrate by treatment with hot dilute acid. Thick line: Same data after subtraction of the collagen background, isolating the signals of bound citrate. (F) Spectrum of calcium citrate, with minimal line broadening (thin line) and broadened (thick line) to match that in (D) and (E). (G) Same as (f) after $40 \mu$ s dipolar dephasing, which selects signals of carbons with weak $\mathrm{C}-\mathrm{H}$ dipolar couplings, i.e. nonprotonated $\mathrm{C}$ and mobile $\mathrm{CH}_{\mathrm{n}}$. (H) Spectrum of bovine bone after $40 \mu \mathrm{s}$ dipolar dephasing. Citrate signals are highlighted by red arrows.



Fig. 2.2. The 76-ppm ${ }^{13} \mathrm{C}$ NMR signal in bone is from an immobile (nonprotonated) $\mathrm{C}-\mathrm{OH}$ group. (A) Wideline ${ }^{1} \mathrm{H}$ spectra associated with $76-\mathrm{ppm}{ }^{13} \mathrm{C}$ signals in bone (solid red line) and sodium citrate dihydrate (dashed orange line), from ${ }^{1} \mathrm{H}^{-13} \mathrm{C}$ wideline-separation (WISE) NMR. Both show no significant motional narrowing. (B) Corresponding ${ }^{1} \mathrm{H}$ spectra of $\mathrm{NCH}$ (protein backbone, dashed line) and $\mathrm{CH}_{3}$ groups. Only the latter, which undergo rotational jumps, exhibit motional narrowing. 

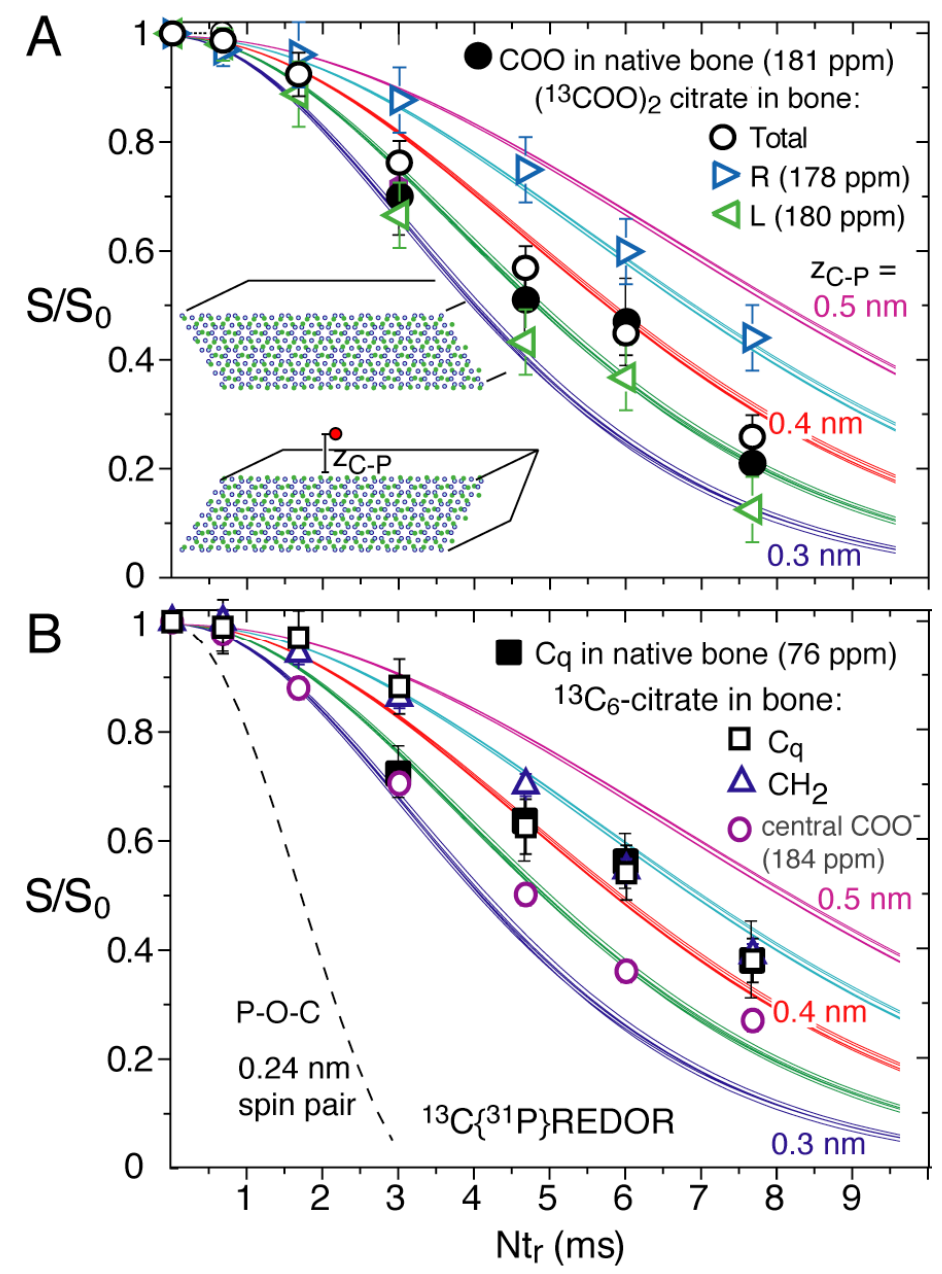

Fig. 2.3. Determination of distances of citrate carbons from bone apatite, measured by ${ }^{13} \mathrm{C}\left\{{ }^{31} \mathrm{P}\right\}$ REDOR NMR. The measured dephasing $\mathrm{S} / \mathrm{S}_{0}$ is plotted along with simulated curves for distances $\mathrm{z}_{\mathrm{C}-\mathrm{P}}$ of $0.3,0.35,0.4,0.45$, and $0.5 \mathrm{~nm}$ of ${ }^{13} \mathrm{C}$ from the first ${ }^{31} \mathrm{P}$ layer at the interface (see inset schematic and Fig. 2.S1). (A) Filled black circles: carboxylate resonance at $181 \mathrm{ppm}$ in native bovine bone; open circles: sum of terminal ${ }^{13} \mathrm{COO}^{-}$groups in ${ }^{13} \mathrm{C}$-labeled citrate absorbed into bovine bone. Triangles pointing right and left: Dephasing of 178- and 180-ppm ${ }^{13} \mathrm{COO}^{-}$citrate signals, respectively. (B) Data for the quaternary C-OH of citrate in bone (filled black squares), as well as quaternary $\mathrm{C}-\mathrm{OH}\left(\mathrm{C}_{\mathrm{q}}\right.$, open squares), $\mathrm{CH}_{2}$ (open blue triangles) and the central $\mathrm{COO}^{-}$(open purple circles) in ${ }^{13} \mathrm{C}$-labeled citrates absorbed into bovine bone. Error margins were determined from the signal-to-noise ratios in the REDOR spectra. The dashed curve is for $\mathrm{a}^{31} \mathrm{C}-{ }^{31} \mathrm{P}$ spin pair (two-bond distance of 0.24 $\mathrm{nm})$ as in phosphocitrate. 


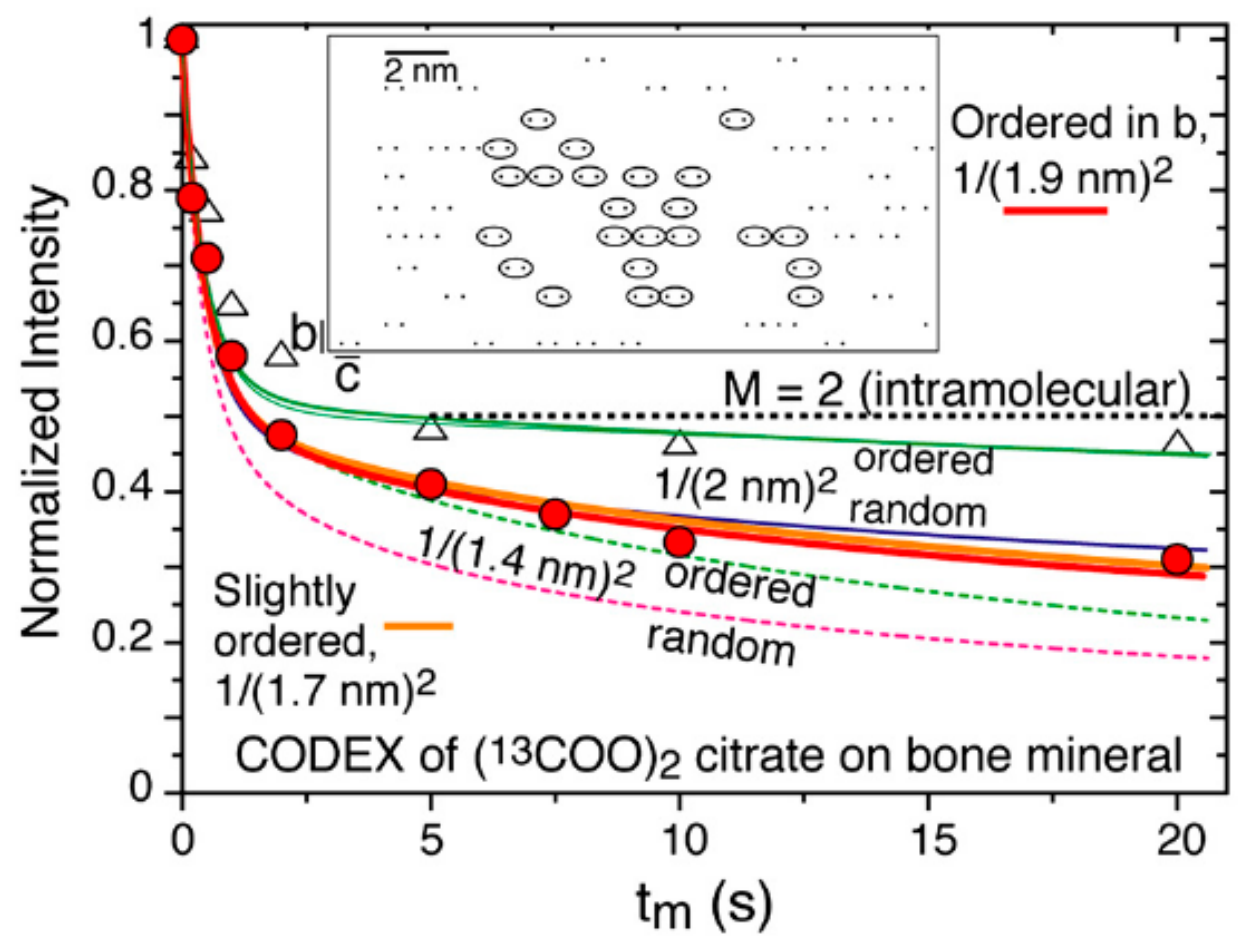

Fig. 2.4. Distances between apatite-bound citrate molecules, probed by CODEX ${ }^{13} \mathrm{C}$ NMR with ${ }^{13} \mathrm{C}$ spin exchange during the time $\mathrm{t}_{\mathrm{m}}$. Data points (red circles) for citrate with terminal ${ }^{13} \mathrm{COO}$ groups on purified bone mineral are compared with fit curves for seven different citrate distributions (Fig. 2.S2). Data points for 2.5 times less citrate, with little intermolecular spin exchange, are shown for reference as open triangles. Inset: One of the best-fit distributions of ${ }^{13} \mathrm{C}$ spins (black dots), with some citrate molecules identified by ellipses. 


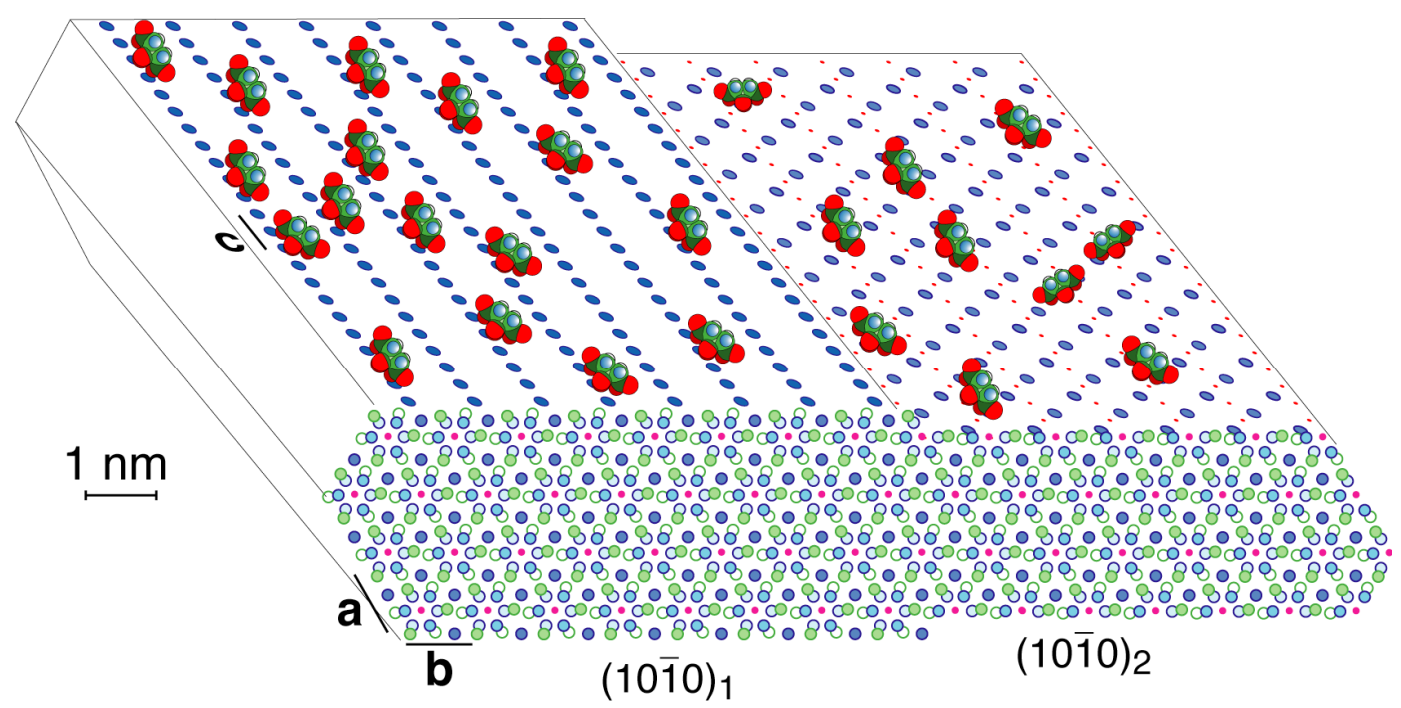

Fig. 2.5. Schematic of apatite-bound citrate (with oxygen of carboxylates in red) interacting with $\mathrm{Ca}^{2+}$ on two surfaces of high morphological importance of an idealized bone apatite nanocrystal, at a realistic citrate surface density of ca. $1 /(2 \mathrm{~nm})^{2}$. Calcium ions are blue filled circles on top and front surfaces, $\mathrm{P}$ is green (omitted on the top surfaces), $\mathrm{OH}^{-}$ions are pink dots, while phosphate oxygen is omitted for clarity. The hexagonal crystal structure projected along the c-axis (with greater depth of atoms indicated by lighter shading) shown in front reveals various layers of phosphate and calcium ions. 


\title{
Supporting Information
}

for

\section{Strongly bound citrate stabilizes the apatite nanocrystals in bone}

\author{
Y.-Y. Hu, A. Rawal, K. Schmidt-Rohr
}

\section{Multi-spin Analysis of NMR Distance Measurements}

${ }^{13} \mathrm{C}\left\{{ }^{31} \mathrm{P}\right\}$ REDOR analysis of citrate distances from apatite. ${ }^{13} \mathrm{C}-{ }^{31} \mathrm{P}$ dipolar coupling measurements by ${ }^{13} \mathrm{C}\left\{{ }^{31} \mathrm{P}\right\}$ REDOR NMR can provide insights into the distances of various citrate carbons from the apatite surface. Figure $2.3 A$ and $B$ shows ${ }^{13} \mathrm{C}\left\{{ }^{31} \mathrm{P}\right\}$ REDOR data for all five spectrally resolved carbon sites in citrate bound to apatite in bone. While many systems studied by REDOR can be approximated in terms of two or few interacting spins, a ${ }^{13} \mathrm{C}$ spin near an apatite surface experiences the dipolar fields of many ${ }^{31} \mathrm{P}$ nuclear magnets. The initial decay depends on the second moment of the ${ }^{13} \mathrm{C}-{ }^{31} \mathrm{P}$ couplings

$$
\mathrm{M}_{2}=\Sigma_{\mathrm{n}} \omega_{\mathrm{C}-\mathrm{P}, \mathrm{n}}{ }^{2}=\Sigma_{\mathrm{n}} \mathrm{C}_{\mathrm{n}}(\alpha, \beta, \gamma) 1 / \mathrm{r}_{\mathrm{C}-\mathrm{P}, \mathrm{n}}{ }^{6}
$$

where $r_{C-P, n}$ is the distance between the carbon and the $n^{\text {th }}{ }^{31} P$ spin, and $C_{n}(\alpha, \beta, \gamma)$ is a purely orientation-dependent prefactor. At sufficiently large distances of the ${ }^{13} \mathrm{C}$ from the apatite surface $\left(\mathrm{z}_{\mathrm{C}-\mathrm{P}}>1 \mathrm{~nm}\right)$, the second moment depends only on the height $\mathrm{z}_{\mathrm{C}-\mathrm{P}}$ above the top phosphate layer, independent of the lateral (x-y) position of the ${ }^{13} \mathrm{C}$. However, when the height $\mathrm{z}_{\mathrm{C}-\mathrm{P}}$ is not much larger than the average P-P distance of $0.45 \mathrm{~nm}$ in apatite, the $\mathrm{x}-\mathrm{y}$ position of the ${ }^{13} \mathrm{C}$ spin needs to be taken into account. Figure 2.S1A shows a contour plot of the distance-only heteronuclear second moment

$$
\mathrm{M}_{2 \mathrm{r}}=\Sigma_{\mathrm{n}} 1 / \mathrm{r}_{\mathrm{C}-\mathrm{Pn}}{ }^{6}
$$

as a function of ${ }^{13} \mathrm{C}$ position at a fixed height of $\mathrm{z}_{\mathrm{C}-\mathrm{P}}=0.35 \mathrm{~nm}$ above a $(10 \overline{1} 0)_{1}$ hydroxyapatite surface. It shows pronounced maxima above ${ }^{31} \mathrm{P}$ (of slightly different height 
for two types of phosphate at different depths from the apatite surface) and wide flat regions around the $\mathrm{Ca}$ ions. Negatively charged citrate is expected to be repelled by the negatively charged phosphate and rather bound to calcium. Therefore, the regions of high $\mathrm{M}_{2 \mathrm{r}}$ near ${ }^{31} \mathrm{P}$ can mostly be disregarded. In addition, the regions of high $\mathrm{M}_{2 \mathrm{r}}$ account for only a small fraction of the total area, see the histogram of $\mathrm{M}_{2 \mathrm{r}}$ in Fig. 3.S1B. Calcium ions are located $0.05 \mathrm{~nm}$ below the top phosphate layer, so $\mathrm{z}_{\mathrm{C}-\mathrm{Ca}}=\mathrm{z}_{\mathrm{C}-\mathrm{P}}+0.05 \mathrm{~nm}$.

Figure S1C shows simulated REDOR curves and data from Fig. $2.3 B$ for the 15 positions B1 - B5, C1 - C5, and D1 - D5 marked in Fig. 3.S1A and $\mathrm{z}_{\mathrm{C}-\mathrm{P}}$ values between 0.3 and $0.5 \mathrm{~nm}$. The height of the ${ }^{13} \mathrm{C}$ nucleus above calcium is larger by $0.05 \mathrm{~nm}$. The data of Fig. 3.3 show that the central and one terminal COO group are closest to the surface, while the other terminal carboxylate is at a $0.1-\mathrm{nm}$ greater height but still close to apatite. In the simulations of the REDOR curves, the second moment with REDOR angular dependencies of all the ${ }^{31} \mathrm{P}_{-}{ }^{13} \mathrm{C}$ couplings was calculated for $>3000$ crystallite orientations, via orientational averaging of the rotor axis relative to the crystallite normal, and rotation of the $\mathrm{B}_{0}$ field around the rotor axis (with the fixed magic angle between them), as outlined in ref. (1). The total dephasing curve is a superposition, for various rotor-axis and $\mathrm{B}_{0}$-field orientations, of Gaussians $\exp \left(-\mathrm{M}_{2}\left(\mathrm{Nt}_{\mathrm{r}}\right)^{2} / 2\right)$, with the second moment $\mathrm{M}_{2}$ evaluated based on the internuclear distances and the REDOR angular dependences of the individual C-P couplings at the given rotor axis and $\mathrm{B}_{0}$ orientation. The simulation program was validated using a spin-pair model geometry.

Calculation of the citrate density on bone mineral. The area density of citrate on apatite in bone and bound to bone mineral can be calculated from the known structural parameters of the materials. The 3-nm thick nanocrystals in bone, alternating with 3-nm thick collagen layers, have a surface-to-sample-volume ratio of

$$
\mathrm{S} / \mathrm{V}_{\text {bone }}=2 \mathrm{~A} /[(3+3) \mathrm{nm} \mathrm{A}]=0.33 / \mathrm{nm}=0.3310^{9} / \mathrm{m}
$$

and the nanocomposite has an average density of

$$
\rho_{\text {ave }}=m_{\text {bone }} / V_{\text {bone }}=\left(\rho_{\mathrm{A}} V_{\mathrm{A}}+\rho_{\mathrm{B}} \mathrm{V}_{\mathrm{B}}\right) / \mathrm{V}_{\text {bone }}=\rho_{\mathrm{A}} \mathrm{V}_{\mathrm{A}} / \mathrm{V}_{\text {bone }}+\rho_{\mathrm{B}} \mathrm{V}_{\mathrm{B}} / \mathrm{V}_{\text {bone }}
$$




$$
=(1.15+3.2) \times 0.5=2.2 \mathrm{~g} / \mathrm{cm}^{3}=2.2 \times 10^{6} \mathrm{~g} / \mathrm{m}^{3}
$$

calculated from 50:50 volume fractions and densities of collagen $\left(1.15 \mathrm{~g} / \mathrm{cm}^{3}\right)$ and apatite $\left(3.2 \mathrm{~g} / \mathrm{cm}^{3}\right)$. From these quantities, the specific surface area (per mass) of bone can be calculated as

$$
\begin{aligned}
\mathrm{S} / \mathrm{m}_{\text {tot }} & =\left(\mathrm{S} / \mathrm{V}_{\text {tot }}\right) /\left(\mathrm{m}_{\mathrm{tot}} / \mathrm{V}_{\text {tot }}\right)=\left(\mathrm{S} / \mathrm{V}_{\mathrm{tot}}\right) / \rho_{\text {ave }} \\
& =0.33 \times 10^{9} / \mathrm{m} /\left(2.2 \times 10^{6} \mathrm{~g} / \mathrm{m}^{3}\right)=150 \mathrm{~m}^{2} / \mathrm{g}=150 \times 10^{18} \mathrm{~nm}^{2} /(\mathrm{g} \text { bone })
\end{aligned}
$$

Also, according to wet chemistry and our spectroscopic analysis, $1 \mathrm{~g}$ of bone contains 1.2 $\mathrm{wt} \%=12 \mathrm{mg}$ citrate, which corresponds to $0.012 \mathrm{~g} / 189 \mathrm{~g} / \mathrm{mol}=6.3 \times 10^{-5} \mathrm{~mol}$ of citrate per $\mathrm{g}$ of bone, or $\mathrm{n}_{\text {citr }} / \mathrm{m}_{\text {tot }}=6.3 \times 10^{-5} \times 6 \times 10^{23}=3.8 \times 10^{19}$ citrate molecules $/(\mathrm{g}$ bone $)$. The area density is thus

$$
\left(\mathrm{n}_{\text {citr }} / \mathrm{m}_{\mathrm{tot}}\right) /\left(\mathrm{S} / \mathrm{m}_{\mathrm{tot}}\right)=3.8 \times 10^{19} /\left(150 \times 10^{18} \mathrm{~nm}^{2}\right)=0.25 / \mathrm{nm}^{2}=1 /(2 \mathrm{~nm})^{2}
$$

The density of ${ }^{13} \mathrm{C}$-labeled citrate on the NuOss ${ }^{\mathrm{TM}}$ bone mineral, with a measured specific surface area of

$$
\mathrm{S} / \mathrm{m}_{\mathrm{tot}}=60 \mathrm{~m}^{2} / \mathrm{g}
$$

can be calculated from the $0.8 \mathrm{mg}$ of citrate, determined by NMR calibrated on neat citrate, per $0.19 \mathrm{~g}$ of bone mineral in the same sample. The total bone-mineral surface area in that sample is

$$
\mathrm{S}=\left(\mathrm{S} / \mathrm{m}_{\mathrm{tot}}\right) \mathrm{m}_{\mathrm{tot}}=60 \mathrm{~m}^{2} / \mathrm{g} * 0.19 \mathrm{~g}=11.4 \mathrm{~m}^{2}=11.4 \times 10^{18} \mathrm{~nm}^{2}
$$

The $0.8 \mathrm{mg}$ of citrate correspond to $0.0008 \mathrm{~g} / 189 \mathrm{~g} / \mathrm{mol}=4.2 * 10^{-6} \mathrm{~mol}$ and thus $\mathrm{n}_{\text {citr }}=4.2 \mathrm{x}$ $10^{-6} \times 6 \times 10^{23}=2.54 \times 10^{18}$ citrate molecules. The area density of citrate on bone mineral is thus

$$
\mathrm{n}_{\text {citr }} / \mathrm{S}=2.54 \times 10^{18} / 11.4 \times 10^{18} \mathrm{~nm}^{2}=0.22 / \mathrm{nm}^{2}=1 /(2 \mathrm{~nm})^{2}
$$


in good agreement with the value for bone obtained above.

CODEX ${ }^{13} \mathbf{C}$ NMR simulations. The CODEX NMR data of citrate with ${ }^{13} \mathrm{C}$-labeled terminal $\mathrm{COO}^{-}$groups shown in Fig. 3.4 were fitted by simulating the spin exchange between $600{ }^{13} \mathrm{C}$ spins in half as many citrate molecules, distributed on a flat rectangular surface of 10 $\mathrm{nm}$ width and ca. $100 \mathrm{~nm}$ length. The spin exchange is characterized by an exchange matrix $\Pi$, with off-diagonal elements

$$
\Pi_{\mathrm{nm}}=0.5 \pi\left[2 \pi 7.5 \mathrm{kHz} 10^{-3} \mathrm{~nm}^{3} / \mathrm{r}_{\mathrm{nm}}^{3}\right]^{2}\left\langle\left(P_{2}(\cos \theta)\right)^{2}\right\rangle \mathrm{F}(0)
$$

reflecting the coupling between ${ }^{13} \mathrm{C}$ spins $n$ and $m$. The value of $\mathrm{F}(0)=0.023 \mu$ s is calibrated by matching the initial decay due to the $0.5-\mathrm{nm}$ intramolecular spin-pair coupling, and is close to literature values for ${ }^{13} \mathrm{COO}$ groups between 0.025 and $0.044 \mathrm{~ms}$ (2). The diagonal element $\Pi_{\mathrm{nn}}$ is the negative of the sum of the $n^{\text {th }}$ column of off-diagonal elements.

The pointed brackets of $\left\langle\left(P_{2}(\cos \theta)\right)^{2}\right\rangle$ indicate the time averaging, due to magic-angle spinning, of the orientation dependence of the dipolar interaction, which depends on the instantaneous angle $\theta$ between the $C^{n}-C^{m}$ internuclear vector and the external $B_{0}$ field. This average depends on the angle $\beta$ between the internuclear vector and the rotor axis. Rather than using only the $\beta$-averaged value $(0.2)$ of $\left\langle\left(P_{2}(\cos \theta)\right)^{2}\right\rangle$, we calculated $\left\langle\left(P_{2}(\cos \theta)\right)^{2}\right\rangle$ as a function of $\cos \beta$ numerically and took the variation of $\left\langle\left(P_{2}(\cos \theta)\right)^{2}\right\rangle$ into account by selecting a random value of $\cos \beta$ between -1 and +1 for each internuclear vector $r_{n m}$ and using the corresponding value of $\left\langle\left(P_{2}(\cos \theta)\right)^{2}\right\rangle$ in the calculated value of $\Pi_{\mathrm{nm}}$ according to the equation above. This provides the same variation of the squared coupling frequencies as full powder averaging and reproduces the observed non-exponential decays (2) better than simulations with a fixed value of $\left\langle\left(P_{2}(\cos \theta)\right)^{2}\right\rangle$.

The normalized CODEX signal $S_{\text {CODEX }}(t)$, which is the fraction of the magnetization remaining on the spin on which it started out, averaged over all spins, is calculated from the 
standard solution for the $N$-component vector $\mathbf{M}$ of the z-magnetization values of the $N$ exchanging spins

$$
\mathbf{M}(\mathrm{t})=\exp (\Pi \mathrm{t}) \mathbf{M}(0)
$$

as

$$
\mathrm{S}_{\text {CODEX }}(\mathrm{t})=\sum_{n=1}^{N}\left(\exp (\Pi \mathrm{t}) \mathbf{e}_{\mathrm{n}}\right)_{\mathrm{n}}=\operatorname{tr}(\exp (\Pi \mathrm{t}))
$$

In the intermediate step, $\mathbf{e}_{\mathrm{n}}$ is the unit vector with elements $\left(\mathbf{e}_{\mathrm{n}}\right)_{\mathrm{m}}=\delta_{\mathrm{nm}}$ (i.e. 1 for $\mathrm{n}=\mathrm{m}$ and 0 otherwise), representing an initial state $\mathbf{M}(0)=\mathbf{e}_{\mathrm{n}}$ with magnetization only on spin $n$. For a sufficiently long dephasing and refocusing time $\mathrm{Nt}_{\mathrm{r}}$, the detected CODEX signal from that spin is proportional to the magnetization $M_{n}(t)=\left(\exp (\Pi t) \mathbf{e}_{n}\right)_{n}$ remaining on that same spin $n$. The sum over $n$ provides the summation of the contributions from all $N^{13} \mathrm{C}$ spins; the trace operation (sum of diagonal elements) on the right-hand side of the equation achieves this in a particularly convenient way.

The simulated CODEX curves depend on the density and positional ordering of the citrate molecules. The ${ }^{13} \mathrm{C}$ distributions corresponding to the seven fit curves in Fig. 3.4 are displayed in Fig. 3.S2. Partial positional ordering is achieved by increasing the excluded area around the center of each molecule. Minimal ("random") ordering is generated by an excluded-area diameter of $\mathrm{d}_{\mathrm{excl}}=0.8 \mathrm{~nm}$ that corresponds to the diameter of a citrate molecule. For an area density of $1 / \mathrm{d}_{\mathrm{cc}}{ }^{2}$ moderate ordering was obtained with $\mathrm{d}_{\mathrm{excl}}=0.6 \mathrm{~d}_{\mathrm{cc}}$, and partial ordering with $\mathrm{d}_{\mathrm{excl}}=0.75 \mathrm{~d}_{\mathrm{cc}}$. A higher than average $\left(>1 /(2 \mathrm{~nm})^{2}\right)$ citrate density is possible for the citrate-covered apatite surfaces, if other surfaces have little or no citrate bound. For instance, the average density of $1 /(2 \mathrm{~nm})^{2}$ can be obtained by combining $50 \%$ of surfaces at $1 /(1.4 \mathrm{~nm})^{2}$ (which yield signal) and $50 \%$ of surfaces without any citrate (which do not contribute to the measured signal). 


\section{References}

1. Schmidt-Rohr K, Rawal A, Fang XW (2007) A new NMR method for determining the particle thickness in nanocomposites, using $\mathrm{T}_{2 \mathrm{H}}$-selective $\mathrm{X}\left\{{ }^{1} \mathrm{H}\right\}$ recoupling. $J$. Chem. Phys. 126: 054701/054701-054716.

2. Luo W, Hong M (2006) Determination of the oligomeric number and intermolecular distances of membrane protein assemblies by anisotropic H-1-driven spin diffusion NMR spectroscopy. J. Am. Chem. Soc. 128: 7242-7251.

\section{Figures}

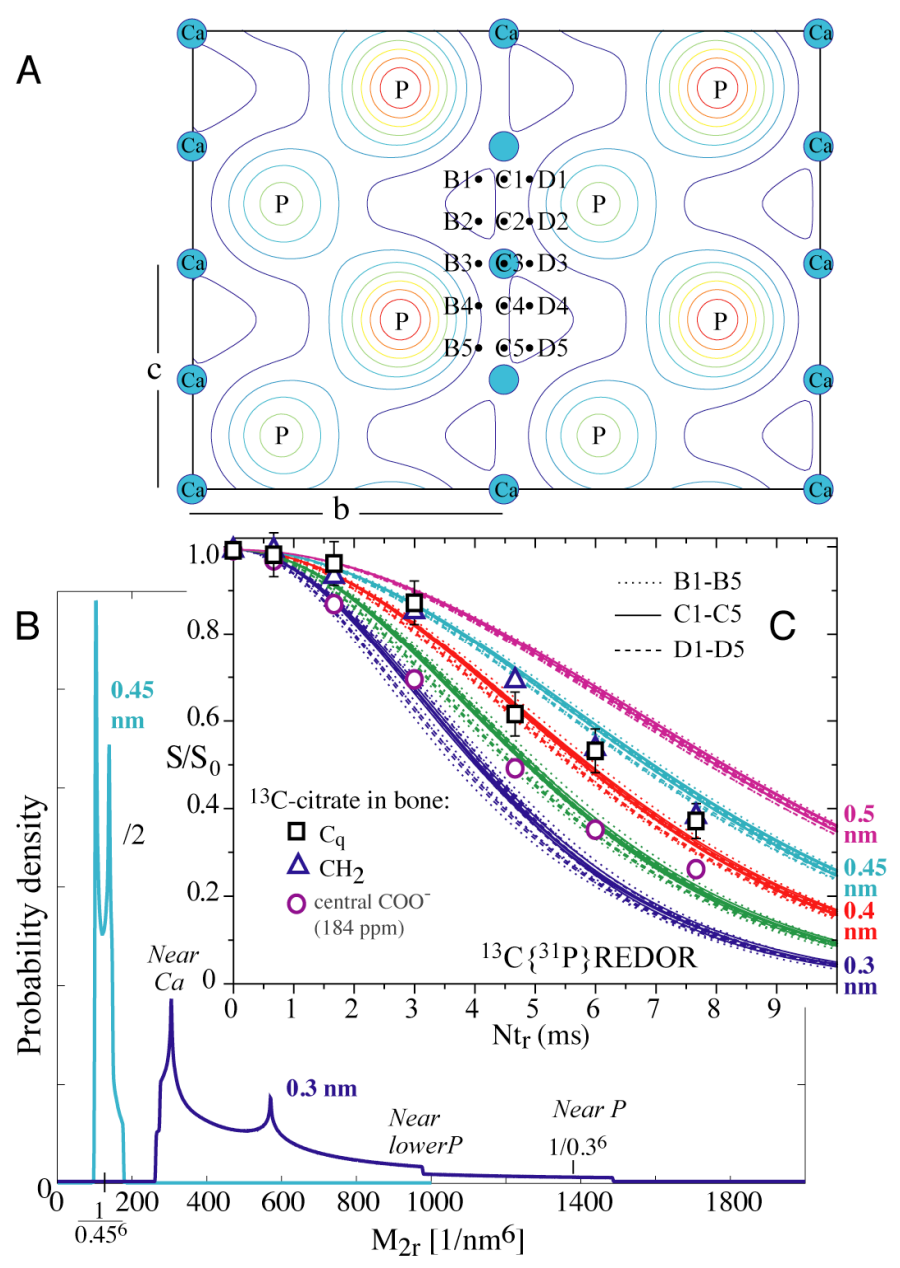

Fig. 2.S1. Analysis of parameters in ${ }^{13} \mathrm{C}\left\{{ }^{31} \mathrm{P}\right\}$ REDOR simulations for citrate carbons in bone. (A) Contour plot of the distance-only second moment $\mathrm{M}_{2 \mathrm{r}}$, i.e. essentially the sum of the squares of the ${ }^{13} \mathrm{C}-{ }^{31} \mathrm{P}$ dipolar couplings, calculated as a function of ${ }^{13} \mathrm{C}$ position at a fixed height $\mathrm{z}_{\mathrm{C}-\mathrm{P}}=0.3 \mathrm{~nm}$ above the top phosphate layer in apatite. Apart from pronounced maxima above the $\mathrm{P}$ atoms, wide 
areas with similar dipolar couplings are seen, in particular surrounding the calcium atoms (light blue circles). Likely positions of the five carbons in citrate, spaced by the projected C-C distance of 0.125 $\mathrm{nm}$ in an all-trans chain, are indicated along the c-axis as C1-C5. Corresponding positions laterally displaced by $0.1 \mathrm{~nm}$ are marked B1-B5 and D1-D5. (B) Corresponding "histogram" of distance-only second moments $\mathrm{M}_{2 \mathrm{r}}$. The large $\mathrm{M}_{2 \mathrm{r}}$ values corresponding to positions above $\mathrm{P}$ are seen to be rare. The narrower histogram for a 0.45 -nm height above the top phosphorus layer is also shown. (C) Data points of exchanged ${ }^{13} \mathrm{C}$-labeled citrate in bone (symbols as in Fig. $3 B$ ) and simulated REDOR dephasing curves for the fifteen points marked B1 - D5 in a) at the indicated $\mathrm{z}_{\mathrm{C}-\mathrm{P}}$ heights of ${ }^{13} \mathrm{C}$ above the top phosphorus layer. C1-C5: full lines; B1-B5: dotted lines; D1-D5: dashed lines.

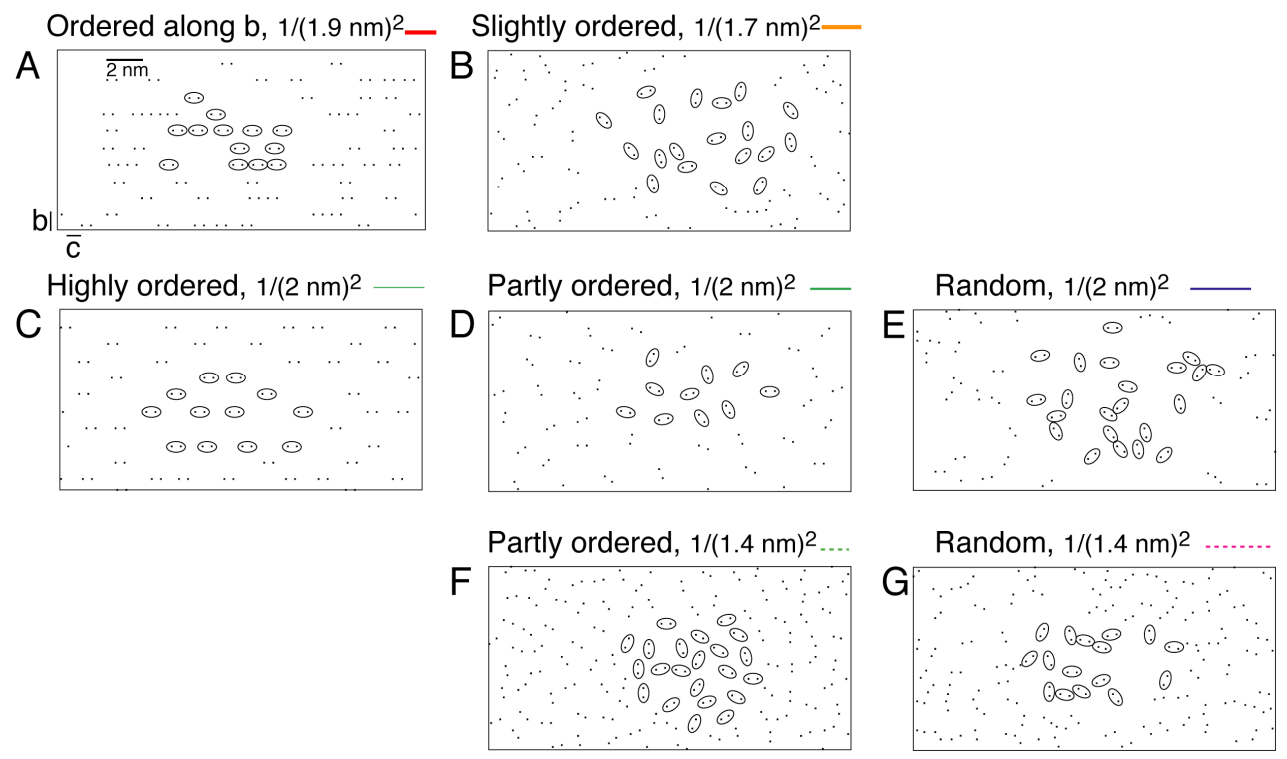

Fig. 2.S2. Distributions of terminal $-{ }^{13} \mathrm{COO}$ groups of citrate (each ${ }^{13} \mathrm{C}$ is marked as a small black square, and some molecules are indicated by elliptical outlines) for the seven fit curves in Fig. 4. (A) Best fit, for an area density of $1 /(1.9 \mathrm{~nm})^{2}$ and positional ordering in one dimension. (B) Best fit, for an area density of $1 /(1.7 \mathrm{~nm})^{2}$ and slight positional ordering. (C) Distribution corresponding to a curve above the experimental data, for $1 /(2 \mathrm{~nm})^{2}$ and significant positional ordering. (D) Distribution corresponding to a curve below the experimental data, for $1 /(2 \mathrm{~nm})^{2}$ and partial positional ordering. (E) Distribution corresponding to a good fit, for $1 /(2 \mathrm{~nm})^{2}$ and minimal positional ordering ("random"). (F) Distribution corresponding to a curve slightly below the experimental data, for $1 /(1.4$ $\mathrm{nm})^{2}$ and partial positional ordering. (G) Distribution corresponding to a curve below the experimental data, for $1 /(1.4 \mathrm{~nm})^{2}$ and minimal positional ordering. 


\title{
Chapter 3
}

\section{Biomimetic Self-assembling Nanocomposites of Block Copolymer and Hydroxyapatite with Nanocrystal Size Controlled by Citrate}

A paper submitted to Journal of the American Chemical Society

Y.-Y. Hu, X. Liu, X. Ma, A. Rawal, T. Prozorov, M. Akinc, S. K. Mallapragada, K. Schmidt-Rohr

Ames Laboratory, Ames, IA 50011

\begin{abstract}
Citrate binds strongly to the surface of calcium phosphate (apatite) nanocrystals in bone and is thought to prevent crystal thickening. In this work, citrate added as a regulatory element enabled molecular control of the size and stability of hydroxyapatite (HAp) nanocrystals in synthetic nanocomposites, fabricated with self-assembling block copolymer templates. The decrease of the HAp crystal size within the polymer matrix with increasing citrate concentration was documented by solid state nuclear magnetic resonance (NMR) techniques and wide-angle X-ray diffraction (XRD), while the shapes of HAp nanocrystals were determined by transmission electron microscopy (TEM). Advanced NMR techniques revealed surface modification of the nanocrystals and, concomitant with the size effects, enhanced mineral interaction with the surrounding organic matrix. The surface-to-volume

ratios determined by NMR spectroscopy and long-range ${ }^{31} \mathrm{P}\left\{{ }^{1} \mathrm{H}\right\}$ dipolar dephasing show that $0,2,10$, and $40 \mathrm{mM}$ citrate changes the thicknesses of the HAp crystals from $3.5 \mathrm{~nm}$ to 2.7 , 2.6, and $2.2 \mathrm{~nm}$, respectively. With citrate concentration comparable to that in body fluids, HAp nanocrystals of similar sizes and morphologies as in avian and bovine bones have been produced.
\end{abstract}

\section{Introduction}

Bone, the primary supporting and protective organ of the mammalian body, is a nanocomposite of nano-sized carbonated apatite crystals and the fibrous protein collagen (ca. 40 vol\% each), with smaller contributions from other proteins and water ${ }^{1-5}$. The integration of the stiff apatite nanocrystals within the tough collagen fibers renders bone lightweight yet 
strong and tough. Human bone undergoes constant dynamic remodeling to repair fatigue damage, such as micro- cracks induced by stress ${ }^{6}$. However, when the damage is beyond the self-restoring ability of bone and severely compromises the quality of life especially in the elderly, therapeutic approaches to regenerate the mineralized tissues are desired. Ideal materials employed in tissue repair therapies should exhibit structural features similar to those in bone, be biocompatible, biodegradable, and bioactive.

Various strategies of tissue engineering have been developed in recent years. ${ }^{7}$ Cell- or protein- based methods simulate the mineralization process in bone $\mathrm{e}^{8-11}$, but the limited availability of materials, immunogenic responses, potential disease transmission, or interference with the therapeutic process curtails their application. ${ }^{7}$ Non-proteinaceous biopolymers, including cellulose, chitosan, and gelatin, have been also employed, but these have fewer apatite-nucleating functional groups such as carboxylate or phosphate moieties, and their properties are not readily tunable. Therefore, the use of synthetic polymers has been an attractive option to provide a scaffold for apatite formation and introduce apatite nucleating reagents. Polymers or polypeptides with acidic groups are favorable in inducing the precipitation of apatite and have been investigated extensively ${ }^{12}$.

A balance between mechanical properties and bioactivity should be considered in biomaterials science; composites of synthetic polymer and calcium phosphate provide a favorable combination. Among various synthesis schemes intended to mimic bone ${ }^{13}$, a bottom-up sol-gel method using inorganic ionic precursors and self-assembling polymers is advantageous since it can control the structure at the molecular level while inducing some hierarchical ordering as seen in bone ${ }^{14}$. With this method, our group has successfully demonstrated the synthesis of organic and inorganic self-assembling nanocomposites using Pluronic (polyethylene oxide-polypropylene oxide triblock copolymer) and its derivatives, polymers coupled to apatite-nucleating peptides, and $\mathrm{K}_{170} \mathrm{~L}_{30}$ diblock copolypeptides as templates that precipitate apatite nanocrystals within the organic matrix ${ }^{15-17}$. Progress has been made to more closely mimic the structure of bone; for instance, hydroxyapatite has been stabilized, and the inorganic content has been increased from $15 \mathrm{wt} \%$ to over $50 \mathrm{wt} \%$, compared to $65 \mathrm{wt} \%$ in bone. Similar research has been pursued by other groups. For instance, Pramanik and Kar synthesized thermoplastic apatite-poly(ether etherketone) 
nanocomposites $^{18}$, Song et al. used crosslinked polymethacrylamide and polymethacryalate hydrogels to template the formation of HAp nanoparticles ${ }^{19}$, and poly(lactic acid), poly(glycolic acid), and poly( $\varepsilon$-caprolactone)/HAp composites also have been constructed for their biodegradability ${ }^{20}$. However, although organic-inorganic nanocomposites have been fabricated, the regulation of the apatite crystal size and morphology has not been discussed. Nevertheless, the crystal dimensions, particularly the crystal thickness is critical to the mechanical properties of bone, in that a thickness of $\sim 3 \mathrm{~nm}$ prevents the propagation of cracks $^{21}$. Also, recent research implied that desirable osteoconductivity could be achieved with synthetic HAp resembling bone minerals in composition, size and morphology ${ }^{22}$.

Many studies of bone biomineralization have focused on identifying proteins promoting or inhibiting the apatite crystal formation, such as a series of Glx-proteins ${ }^{5}$. It has been hypothesized that proteins such as osteocalcin have a mechanism for mineral surface recognition which allows for biological control of the mineral crystal growth ${ }^{23}$. However, a most recent close examination of the interface between collagen and apatite crystals in bone by advanced solid state NMR techniques ${ }^{24}$ has revealed that citrate, a small molecule, is the dominant organic molecule there, tightly bound to apatite and covering the surface with a density of 1 citrate molecule per $4 \mathrm{~nm}^{2}$ (ca. $1 / 6$ of the available area). About $80 \mathrm{wt} \%$ of the total citrate in the body is accumulated in bone. Carboxylate groups are key for regulating the formation of apatite ${ }^{5,25}$ and citrate in bone provides more carboxylate groups than all the proteins other than collagen taken together. In vitro studies have shown that citrate at higher concentrations results in smaller HAp nanocrystals ${ }^{26,27}$. Moreover, citrate stabilizes HAp over other phosphates ${ }^{28}$ and synthetic materials pretreated with citrate give better biocompatibility in tissue repair ${ }^{29,30}$. Despite these studies, the effect of citrate upon the local molecular composition and surface structure is not well understood. This is in part due to the challenge of selectively characterizing mineral surfaces, especially those buried in organic matrices. The surface- and structure- directing effect of small molecules is general to a wide variety of functionally important materials such as zeolites ${ }^{31}$, mesoporous materials ${ }^{32}$ and solgel based electronic materials ${ }^{33}$, in addition to biomaterials.

Inspired by the study of citrate-apatite in bone and in vitro, we have added citrate as a third component in our synthesis of self-assembled Pluronic polymer and HAp 
nanocomposites. This research allows more precise control over the crystal size and morphology of synthetic HAp within a polymer matrix in order to more closely mimic the structure and morphology of nanocrystals in bone. By adjusting the concentration of citrate, we have achieved molecular control over the size of the HAp nanocrystals within the Pluronic polymer matrix. These synthetic nanocomposites have been characterized by transmission electron microscopy (TEM), X-ray diffraction (XRD), small angle neutron scattering (SANS) and advanced solid-state nuclear magnetic resonance (ssNMR)

techniques. ${ }^{31} \mathrm{P}$ NMR has been used for molecular characterization of the calcium phosphate species formed, probe molecular proximities on the nanometer scale, and accurately quantify the surface and core phosphate contents of the nanocrystals. ${ }^{1} \mathrm{H}-{ }^{31} \mathrm{P}$ heteronuclear correlation (HetCor) spectra with ${ }^{1} \mathrm{H}$ spin diffusion provides a powerful method to monitor the formation of organic-inorganic nanocomposites and qualitatively reveal the enhanced surface area of HAp with increasing citrate concentration. ${ }^{1} \mathrm{H}-{ }^{31} \mathrm{P}$ HARDSHIP NMR ${ }^{34}$ has been used to quantitatively determine the crystal size and compare with that in bone, which is often considered the ideal bio-composite. The results obtained from NMR and XRD show how the apatite nanocrystal size decreases with increasing citrate concentration. The citrate concentration was set comparable to that in body fluids to determine whether apatite nanocrystals similarly sized as in natural bone can be generated. TEM has been applied to reveal the morphologies of apatite nanocrystals with varying citrate concentrations, while small angle neutron scattering (SANS) can determine the effect of citrate and HAp nanocrystal formation on the long-range order and morphology of the Pluronic polymer matrix assembly. It is expected that the biocompatibility of biosynthetic materials can be enhanced by the incorporation of citrate within the nanocomposites to produce similarly structured HAp nanocrystals as in bone apatite.

\section{Materials and Methods}

\section{Chemicals.}

The Pluronic F127 block copolymer, poly(ethylene oxide)-b-poly(propylene oxide)b-poly(ethylene oxide) (PEO-PPO-PEO), was purchased from Sigma Aldrich (St. Louis, MO). Its average molecular weight is about 12,600 kDa. An aqueous solution of the polymer 
can self-assemble into micelles at low temperature and concentration ${ }^{15}$, and transform to a viscous gel upon increase in temperature (typically above $25^{\circ} \mathrm{C}$ ) or concentration. All the other chemicals in this study were purchased from Sigma Aldrich or Fisher Scientific, and used without further purification.

\section{Nanocomposite Synthesis.}

The calcium phosphate nanocomposites were prepared using self-assembling polymers as templates as follows: $0.01 \mathrm{~mol}$ of $\mathrm{Ca}\left(\mathrm{NO}_{3}\right)_{2}$ was dissolved in $10 \mathrm{~mL}$ of $30 \mathrm{wt} \%$ Pluronic F127 solution at $4{ }^{\circ} \mathrm{C}$, followed by addition of $0.2 \mathrm{~mL}$ of ammonium citrate at various concentrations and mixed for 2 hours at $4^{\circ} \mathrm{C} .2 \mathrm{~mL}$ of $3 \mathrm{M}\left(\mathrm{NH}_{4}\right)_{2} \mathrm{HPO}_{4}$ was then added. The sample was brought to room temperature and allowed to form a gel with gentle stirring. $\mathrm{NH}_{4} \mathrm{OH}$ was then added to adjust the $\mathrm{pH}$ to $7.5-8$; after 24 hours, the $\mathrm{pH}$ was checked and re-adjusted to 7.5-8; the $\mathrm{pH}$ was further checked and re-adjusted until it remained stable. Four hydrogel samples with citrate concentrations of $0,2,10$, and $40 \mathrm{mM}$ were prepared. The samples were freeze-dried and characterized.

In addition, samples were washed to remove free polymer and ammonium nitrate, as needed for characterization. The sample powder was suspended in pure water, centrifuged, and the supernatant was removed. This procedure was repeated three times in order to minimize the amount of residual free polymer and ammonium nitrate.

\section{Characterization}

XRD. The structure of the freeze-dried and washed samples was investigated by XRD (X'Pert PRO, PANalytical Inc., Westborough, MA,) and analyzed by X'pert Data collector. The diffractometer was operated at $45 \mathrm{kV}$ and $40 \mathrm{~mA} . \mathrm{CuK} \alpha$ radiation with a wavelength of $0.15418 \mathrm{~nm}$ was employed. The scan rate was $0.021^{\circ} / \mathrm{s}$ with a step size of $0.017^{\circ}$ over the range of $10^{\circ} \leq 2 \theta \leq 60^{\circ}$.

TEM. Imaging of the samples was performed without staining with the Tecnai $\mathrm{G}^{2} \mathrm{~F} 20$ Scanning Transmission Electron Microscope (STEM) (FEI Company, Hillsboro OR) equipped with High Angle Annular Dark Field (HAADF) and Energy Dispersive X-ray 
Spectroscopy (EDS) detectors at an operating voltage of $200 \mathrm{KV}$. Twenty microliters of hydroxyapatite suspension were dispersed in $2 \mathrm{~mL}$ of distilled $\mathrm{H}_{2} \mathrm{O}$. A drop of the diluted suspension was placed on a holey carbon-supported copper grid. Multiple areas of each sample were examined to determine the average size of the particles in the nanocomposites, both in bright field TEM mode and in STEM mode.

SANS. Small-angle neutron scattering (SANS) was used to highlight the structure of the Pluronic block copolymer in the nanocomposite gels. $1 \mathrm{~mL}$ deuterium oxide $\left(\mathrm{D}_{2} \mathrm{O}\right)$ was added to about $0.5 \mathrm{~mL}$ gel sample. The polymer phase has higher neutron scattering contrast than the inorganic phase in a deuterated solvent, since the scattering length of ${ }^{1} \mathrm{H}$ is negative while the scattering lengths of all other isotopes involved are positive and of similar magnitude.

The experiments were performed using the $30 \mathrm{~m}$ small angle scattering instrument on beamline NG7 at the National Center for Neutron Research, National Institute of Standards and Technology (NIST), Gaithersburg, MD. A cold neutron source with an average wavelength of $6 \AA$ was used. The samples were sealed in quartz cylindrical cells with a -mm path length. The scattered neutrons were detected by a two-dimensional detector with three different sample-to-detector distances of 13,4 , and $1 \mathrm{~m}$. The scattering vector, q, was varied between $0.004 \AA^{-1}<\mathrm{q}<0.56 \AA^{-1}$, where $\mathrm{q}=(4 \pi / \lambda) \sin (\theta / 2)$, in which $\lambda$ is the neutron wavelength and $\theta$ is the scattering angle. The $\mathrm{I}(\mathrm{q})$ data were placed on an absolute scale in units of $\mathrm{cm}^{-1}$. Data reduction and plots were accomplished by using SANS reduction and analysis macros provided by NIST (http://www.ncnr.nist.gov/programs/sans/data/red_anal.html).

NMR. All the NMR experiments were carried out on a Bruker Biospin DSX-400 spectrometer (Bruker-Biospin, Rheinstetten, Germany) at $400 \mathrm{MHz}$ for ${ }^{1} \mathrm{H}$ and $162 \mathrm{MHz}$ for ${ }^{31} \mathrm{P}$. A Bruker 4-mm double resonance magic-angle spinning (MAS) probehead was used with spinning frequencies of $4.3 \mathrm{kHz}$ for ${ }^{1} \mathrm{H}_{-}{ }^{31} \mathrm{P}$ HetCor and ${ }^{31} \mathrm{P}$ spin diffusion experiments and $6.5 \mathrm{kHz}$ for other experiments. The $90^{\circ}$ pulse length was $4 \mu$ s for ${ }^{1} \mathrm{H}$ and $4.5 \mu$ s for ${ }^{31} \mathrm{P}$. 
A $200 \mathrm{~s}$ recycle delay was used in direct-polarization ${ }^{31} \mathrm{P}$ NMR experiments without or with two rotor periods of gated ${ }^{1} \mathrm{H}^{31} \mathrm{P}$ heteronuclear recoupling. The two-pulse phasemodulation (TPPM) ${ }^{1} \mathrm{H}_{-}-{ }^{31} \mathrm{P}$ heteronuclear decoupling strength was $60 \mathrm{kHz}$ during ${ }^{31} \mathrm{P}$ detection. Hartman-Hahn cross-polarization (HHCP) of $1 \mathrm{~ms}$ was employed for experiments requiring polarization transfer from ${ }^{1} \mathrm{H}$ to ${ }^{31} \mathrm{P} .{ }^{1} \mathrm{H}^{31} \mathrm{P}$ HetCor experiments were carried out with MREV-8 for ${ }^{1} \mathrm{H}-{ }^{1} \mathrm{H}$ homonuclear decoupling and ${ }^{1} \mathrm{H}$ chemical shifts were scaled by 0.47 accordingly during data processing. $160 \mathrm{t}_{1}$ increments of $60 \mu$ s were employed and multiecho detection ${ }^{35}$ was implemented to enhance signal sensitivity. In ${ }^{31} \mathrm{P}$ spin diffusion experiments, a short $\mathrm{CP}$ contact time of $0.1 \mathrm{~ms}$ was employed to generate ${ }^{31} \mathrm{P}$ magnetization selectively in phosphates close to protons with subsequent $0.1-\mathrm{ms}$ and $1-\mathrm{s}{ }^{31} \mathrm{P}$ spin diffusion times. A spin diffusion time of $100 \mathrm{~s}$ was used in a sample without citrate and containing two phosphate phases. The duration of the z-period was incremented in 8 steps of $\mathrm{t}_{\mathrm{r}} / 8=29.1 \mu \mathrm{s}$ in order to cancel dispersive contributions to the spinning sidebands, which would results in baseline distortions. ${ }^{1} \mathrm{H}^{-31} \mathrm{P}$ HARDSHIP experiments ${ }^{34}$ were performed with direct polarization and recoupling times of $0.3,0.6,0.9,2.1,3.0,4.6,6.1,7.7$, and $9.2 \mathrm{~ms}$. Differential $\mathrm{T}_{2}$ relaxation of surface and core phosphate signals in $\mathrm{S}_{0}$ was corrected for as outlined in ref.[34]. The NIST hydroxyapatite with ${ }^{1} \mathrm{H}$ peak at $0.18 \mathrm{ppm}$ and ${ }^{31} \mathrm{P}$ peak at 2.8 ppm was used to calibrate ${ }^{1} \mathrm{H}$ and ${ }^{31} \mathrm{P}$ chemical shifts, respectively.

Avian and bovine bones used in HARDSHIP experiments were extracted from the mid- sections of femur bones from a mature hen and a 4-year-old cow. The surface was cleaned with a scalpel to remove muscle tissue and then washed with a solution of methanol and chloroform to remove lipids. The bone was ground to a powder and lyophilized to remove any traces of solvent and bulk water.

\section{Results and Discussion}

The properties of synthetic nanocomposites are strongly dependent on the mineral size, composition, and the surface structure as well as its interaction with the surrounding organic matrix. Mineralization of an organic matrix increases the mechanical strength provided that the mineral is distributed uniformly and has molecular-level interactions with the templating organic matrix. XRD, TEM, SANS, and NMR are a powerful set of 
complementary techniques that yield information about the size of the mineral nanocrystals as well as the effect of mineralization on the structure of the templating organic polymer. 1D and 2D NMR techniques uniquely enable selective molecular characterization of the nanocrystal surface and core, as well as the mineral-organic interactions, from which insights about the influence of citrate molecules and formation of the nanocomposites can be obtained.

XRD. XRD patterns of the four synthesized samples after freeze drying and after washing are shown in Figures 3.1 and 3.2, respectively. When compared to the pure NIST hydroxyapatite (HAp) pattern, the patterns show similarities and differences. The peaks in the diffraction pattern, in particular the intense bands at approximately $2 \theta=32^{\circ}$ and $2 \theta=$ $26^{\circ}$, confirm that the predominantly mineralized phase is HAp. While the patterns of the synthesized materials differ from those of NIST HAp in showing much broader peaks, they are very similar to those of human bone and dentin. ${ }^{36}$ The broad peak at approximately $2 \theta=$ $32^{\circ}$ is a composite band contributed from three of the most intense peaks of HAp [(211), (112), and (300) planes] ${ }^{17}$. These broad peaks are indicative of the formation of HAp crystals in the low nanometer size range. Additional diffraction peaks seen in Figure 3.1 at $2 \theta=21^{\circ}$ and $2 \theta=24^{\circ}$ were assigned to crystalline polyethylene oxide ${ }^{16}$ which was confirmed by the much reduced intensity of these two peaks after removal of the polymer by washing (see Figure 3.2). Most importantly, it is seen from Figure 3.2 that as the concentration of citrate in the synthesis was increased, the diffraction peaks broadened, which indicates that the size of the HAp nanocrystals is decreased. This is consistent with the NMR results, as discussed in more detail below.

TEM. HAADF-STEM is sensitive to scattered electrons generated in proportion to the atomic number $(Z)$ of the atoms in the specimen. It can be used for obtaining compositional and morphological information of a sample (Z-contrast imaging), where contrast varies roughly as a function of $Z^{2} .{ }^{37,38} \mathrm{It}$ is, therefore, possible to image high- $Z$ species inside a densely populated lower-Z environment of a nanocomposite specimen. Example of such analysis of the unwashed hydroxyapatite samples is shown in Figure 3.3. Dark-field (DF) 
images are obtained with HAADH-STEM at the magnification of 110,000. Here the bright spots correspond to the calcium-rich thicker areas of the nanocomposite. According to Figure 3.3, the sample prepared with the highest concentration of citrate has the shortest and thinnest particles.

Figure 3.4 shows Bright-Field (BF) Transmission Electron Micrographs obtained at a magnification of 88,000. Analysis both in the BF TEM mode and HAADF-STEM mode reveals elongated particles of approximately $5 \mathrm{~nm}$ thickness and a mean particle length of 50 $\mathrm{nm}$. These data are in good agreement with the results from the other techniques.

SANS. Figure 3.5 shows SANS data in a plot of $\log \mathrm{I}(\mathrm{q})$ vs. $\log (\mathrm{q})$ for Pluronic polymerhydroxyapatite nanocomposite gels with different citrate concentrations. Due to the small neutron scattering contrast between $\mathrm{D}_{2} \mathrm{O}$ and calcium phosphate, whose isotopes all have positive lengths of similar magnitudes, the inorganic component is essentially invisible here. This enables selective observation of the structure of the polymer micelles. For all four samples, a pronounced peak is observed at essentially the same $\mathrm{q}_{\max }$ of $3 \mathrm{~A}^{-1}$, which corresponds to a characteristic length scale of ca. $15 \mathrm{~nm}$. This peak was observed previously for the neat hydrated Pluronic material and shown to be due to the self-organization of the triblock copolymer into micelles ${ }^{16}$. The fact that the observed scattering pattern is very similar for the different samples indicates that the nanocrystals as well as the citrate molecules are incorporated into the organic matrix in a manner that does not disrupt the selfassembled structure of the pluronic polymer micelles.

NMR characterization of the synthetic composites. Direct-polarization (DP) 1D solid state ${ }^{31} \mathrm{P}$ NMR spectra yield quantitative site populations of the different phosphate species precipitated in the mineral phase. Figure 3.6, shows the ${ }^{31} \mathrm{P}$ DP NMR spectra of composites synthesized with Pluronic polymer, calcium phosphate, and citrate of different concentrations (bold line). Analysis of spectra reveals two overlapping spectral components that are recognizable in all four samples, which correspond to two different phosphate species. The first is represented by a relatively sharp peak resonating at $\sim 2.8 \mathrm{ppm}$, characteristic of hydroxyapatite (HAp) orthophosphate. $2 \mathrm{D}{ }^{1} \mathrm{H}_{-}{ }^{31} \mathrm{P}$ HetCor NMR further prove it to be HAp 
by its connection to the $\mathrm{OH}^{-1} \mathrm{H}$ resonance in experiments described below. The second phosphate species exhibits a broad peak centered around 2 ppm and with a full width at half maximum (FWHM) of 5 ppm, indicating a disordered phosphate environment. The NMR signal of the phosphate species near protons $\left({ }^{1} \mathrm{H}\right)$ can be selectively enhanced by ${ }^{31} \mathrm{P}\left\{{ }^{1} \mathrm{H}\right\}$ cross polarization (CP). The CP spectra (Figure 3.S1) of the samples show lineshapes different from those in the $\mathrm{DP}{ }^{31} \mathrm{P}$ spectra due to this selective enhancement. In Figure 3.S1 the signal of the disordered phosphate component is selectively enhanced over that of the HAp phosphate species. This indicates that the disordered phosphate component has a closer proximity to ${ }^{1} \mathrm{H}$ than the HAp component.

On the other hand, based on the difference in their distance from the surface and polymer protons (which exhibit strong spin-spin interactions and therefore a short spin-spin relaxation time $\mathrm{T}_{2}$ ), the two phosphate components can be separated by ${ }^{1} \mathrm{H}^{3}{ }^{31} \mathrm{P}$ HARDSHIP NMR experiments, in which ${ }^{31} \mathrm{P}$ signal of the disordered phosphate is dephased (suppressed) by protons with short $\mathrm{T}_{2}$ much faster than that of $\mathrm{HAp}^{34}$. This differential dephasing enables a deconvolution of the overlapping DP spectra of the two phosphate components into two separate components as shown in Figure 3.6 (thin lines).

Proximity of phosphate components. The molecular proximities between different ${ }^{31} \mathrm{P}$ components in the nanocomposite can be probed by ${ }^{31} \mathrm{P}$ spin diffusion. In the spin diffusion process, the ${ }^{31} \mathrm{P}$ magnetization is stored along the $\mathrm{z}$ axis, which is then distributed to nearby nuclei via dipolar couplings. This method allows us to evaluate proximities among the different phosphate species on the 3-nm scale. First, the magnetization is selectively placed on the ${ }^{31} \mathrm{P}$ nuclei of the broad component by short $(0.1 \mathrm{~ms})$ cross polarization from protons (Figure 3.7, thick line), then via the ${ }^{31} \mathrm{P}_{-}{ }^{31} \mathrm{P}$ spin diffusion process, the magnetization spreads to the nearby ${ }^{31} \mathrm{P}$ nuclei. As the spin diffusion progresses, there is a gradual change in the lineshape of the ${ }^{31} \mathrm{P}$ spectra. Beginning with a broad line shape, the spectrum increasingly narrows and eventually, after 1 second of spin diffusion, the whole spectrum (Figure 3.7, thin line) matches the DP spectrum (Figure 3.7, dotted line), indicating that spin diffusion equilibrium is reached. The fact that spin diffusion occurs from the disordered phosphate to the HAp phosphates indicates that these two components are not macroscopically phase 
separated. Indeed, the observed fast 1-s time scale of the ${ }^{31} \mathrm{P}$ spin diffusion between these two kinds of phosphates reveals that they are in close proximity on the 2-nm length scale. Therefore, the disordered component resonating at $2 \mathrm{ppm}$, which is close to surface $\mathrm{H}_{2} \mathrm{O}$ $\left(\mathrm{HPO}_{4}{ }^{2-} / \mathrm{PO}_{4}{ }^{3-}-\mathrm{H}_{2} \mathrm{O}\right)$ as well as the $\mathrm{HAp}$ phosphate species, must be the surface layer of the HAp nanocrystals. On the other hand the relatively ordered, sharp component at $2.8 \mathrm{ppm}$ is the core $\mathrm{PO}_{4}{ }^{3-}$ of HAp nanocrystal, which is similar to the structure in native bone.

Quantification of surface $\mathrm{HPO}_{4}{ }^{2-} / \mathrm{PO}_{4}{ }^{3-}-\mathrm{H}_{2} \mathrm{O}$ and core $\mathrm{PO}_{4}{ }^{3-}$. Quantification of surface and core phosphates of the synthetic HAp in each sample enables monitoring the influence of citrate on the molecular structure of the precipitating mineral phase. This is done by integrating the two components in the deconvoluted DP spectra in Figure 3.6. The results in Table 3.1 show that as the citrate concentration is increased from 0 to $40 \mathrm{mM}$, the amount of surface $\mathrm{HPO}_{4}{ }^{2-} / \mathrm{PO}_{4}{ }^{3-}-\mathrm{H}_{2} \mathrm{O}$ species nearly doubles from $27 \%$ to $52 \%$, while the core $\mathrm{PO}_{4}{ }^{3-}$ content concomitantly decreases from $73 \%$ to $48 \%$, which indicates that the specific surface area increases. In other words, the addition of citrate results in smaller HAp crystals, with higher concentration of surface specific phosphate species. In particular, at a citrate concentration of $2 \mathrm{mM}$, similar to the average citrate concentration in body fluids, 59\% core $\mathrm{PO}_{4}{ }^{3-}$ and $41 \%$ surface $\mathrm{HPO}_{4}{ }^{2-} / \mathrm{PO}_{4}{ }^{3-}-\mathrm{H}_{2} \mathrm{O}$ are produced, which is very similar to that seen in chicken bone, where $65 \%$ is core $\mathrm{PO}_{4}{ }^{3-}$ and $35 \%$ surface $\mathrm{HPO}_{4}{ }^{2-} / \mathrm{PO}_{4}{ }^{3-}-\mathrm{H}_{2} \mathrm{O}$. The tuning of nanocrystal size and surface fraction is expected to have important consequences for their solubility behavior and the mineral-organic interaction.

Stabilization of HAp by citrate. Repeated syntheses of all samples mentioned above were analyzed in order to evaluate the reproducibility of the results. Syntheses with ammonium citrate as an ingredient always yielded HAp, while without citrate, other phosphate phases were found to form sometimes, which indicates that citrate uniquely stabilizes HAp crystals over other calcium phosphates and does so effectively at very small crystal thicknesses. This is consistent with the effect that citrate has of destabilizing phases such as brushite and converting them into $\mathrm{HAp}^{30}$. To give an example of a non-HAp phosphate resulting from one of our syntheses with $0 \mathrm{mM}$ citrate, Figure 3.8 shows the ${ }^{31} \mathrm{P} C P / \mathrm{MAS}$ spectrum with $0.1 \mathrm{~ms}$ 
contact time (thick line). It consists of two phosphate components; one is HAp with core $\mathrm{PO}_{4}{ }^{3-}$ resonating at $2.8 \mathrm{ppm}$ and a broadband of surface $\mathrm{HPO}_{4}{ }^{2-} / \mathrm{PO}_{4}{ }^{3-}-\mathrm{H}_{2} \mathrm{O}$. The other resonates at $1.3 \mathrm{ppm}$ with strong spinning sidebands, which is the signature of a local $\mathrm{PO}_{4}$ environment with strong deviations from tetrahedral symmetry. ${ }^{1} \mathrm{H}^{-}{ }^{31} \mathrm{P}$ HetCor results show that this $1.3 \mathrm{ppm}{ }^{31} \mathrm{P}$ correlates with ${ }^{1} \mathrm{H}$ resonating around $10 \mathrm{ppm}(\mathrm{POH})$ and $\mathrm{H}_{2} \mathrm{O}$ with strong hydrogen bonding at $7 \mathrm{ppm}$ (Figure 3.S2). Both ${ }^{31} \mathrm{P}$ and ${ }^{1} \mathrm{H}$ spectra are typical of $\mathrm{HPO}_{4}{ }^{2-}$ surrounded by bound $\mathrm{H}_{2} \mathrm{O} .{ }^{31} \mathrm{P}$ spin diffusion in Figure 3.8 (thin line) show proximity only of surface $\mathrm{HPO}_{4}{ }^{2-} / \mathrm{PO}_{4}{ }^{3-}-\mathrm{H}_{2} \mathrm{O}$ and core $\mathrm{PO}_{4}{ }^{3-}$ of $\mathrm{HAp}$. Even after $100 \mathrm{~s}$ spin diffusion, the CP spectrum (thin line) does not match the DP spectrum (dashed line); note in particular the lack of sideband equilibration and mismatch of the right shoulder and the center band. This indicates that in this sample, some of the $\mathrm{HPO}_{4}{ }^{2-}$ with ${ }^{31} \mathrm{P}$ resonating at 1.3 ppm is a separate phase from HAp and these two phosphates are separated on the nanoscale. In the presence of citrate, such non-HAp phases were not observed.

${ }^{1} \mathbf{H}$ spectra of phosphate environments. The ${ }^{1} \mathrm{H}-{ }^{31} \mathrm{P}$ HetCor spectra with ${ }^{1} \mathrm{H}$ homonuclear decoupling and minimal ${ }^{1} \mathrm{H}$ spin diffusion time of $0.05 \mathrm{~ms}$ (Figure 3.9, top row) reveal the immediate proton environment around specific ${ }^{31} \mathrm{P}$, which helps confirm the identity of phosphates. The ${ }^{1} \mathrm{H}$ spectra extracted at $2.8 \mathrm{ppm}$ of ${ }^{31} \mathrm{P}$ of the sample containing $0 \mathrm{mM}$ citrate (Figure 3.9a, left column, top row) exhibit predominantly the $\mathrm{OH}^{-}$proton peak at 0.2 ppm; this confirms the assignment of the $2.8 \mathrm{ppm}$ peak in the ${ }^{31} \mathrm{P}$ spectra to HAp. The relatively weak ${ }^{1} \mathrm{H}$ peak at $\sim 7 \mathrm{ppm}$ is from bound water at the surface of HAp. The ${ }^{1} \mathrm{H}$ spectrum (Figure 3.9a, right column, top row) taken at $1 \mathrm{ppm}$ of ${ }^{31} \mathrm{P}$ to minimize the interference from HAp represents the ${ }^{1} \mathrm{H}$ environment for the broad phosphate component. It shows reduced intensity of the $\mathrm{OH}^{-}$peak and increased intensity of the bound water signal (more distinctively seen in Fig 9b, top row). Furthermore, additional intensity at $\sim 11 \mathrm{ppm}$ and $\sim 4$ ppm belonging to surface $\mathrm{HPO}_{2}^{2-}$ and organic $\mathrm{OCH}_{2}$ species is seen, respectively. In particular, the signal for the $\mathrm{OCH}_{2}$ species from the hydrophilic PEO segment of the Pluronic polymer confirms that the disordered phosphate is the surface component of the HAp nanocrystal. The broad $\mathrm{OCH}_{2}$ signal indicates that the PEO segment is immobilized due to 
favorable interactions with the mineral surface, potentially mediated by hydrogen bonding. This indicates formation of an intimately mixed nanocomposite system.

Nanocomposite formation. The formation of the nanocomposite is more clearly probed by monitoring the ${ }^{1} \mathrm{H}$ spin diffusion from polymer protons to the mineral phase in $2 \mathrm{D}{ }^{1} \mathrm{H}-{ }^{31} \mathrm{P}$ HetCor experiments ${ }^{14}$. With increasing ${ }^{1} \mathrm{H}$ spin diffusion time, the presence of the polymer $\mathrm{OCH}_{2}(4 \mathrm{ppm})$ and $\mathrm{CH}_{3}(1.5 \mathrm{ppm})$ peaks in the ${ }^{1} \mathrm{H}^{3}{ }^{31} \mathrm{P}$ HetCor experiments (Figure 3.9 and $3 . \mathrm{S} 3, \mathrm{t}_{\mathrm{SD}}=5,50$, and $500 \mathrm{~ms}$ ) proves that in all four samples the mineral is in nanometerscale contact with the organic matrix. The addition of citrate enables formation of crystals that are so small that even within a short ${ }^{1} \mathrm{H}$ spin diffusion time of only $0.05 \mathrm{~ms}$, contact between the inorganic phosphate and Pluronic polymer is indicated by the appearance of the rigid $\mathrm{OCH}_{2}$ proton peak at 4 ppm. This is most apparent in Figure 3.9(b) for the nanocomposite synthesized with the highest citrate concentration $(40 \mathrm{mM})$. At longer spin diffusion times, one can see the ${ }^{1} \mathrm{H}$ peak at $1.3 \mathrm{ppm}$, from $\mathrm{CH}_{3}$ of the hydrophobic PPO segments of the Pluronic polymer appear. At the same spin diffusion times, the magnetization spreads to the relatively mobile $\mathrm{OCH}_{2} / \mathrm{OCH}$, and $\mathrm{NH}_{4}{ }^{+}$(from the starting material $\left.\left(\mathrm{NH}_{4}\right)_{2} \mathrm{HPO}_{4}\right)$ adsorbed on the surface of HAp and their signals appear at $4 \mathrm{ppm}$ and $7.3 \mathrm{ppm}$, respectively. Meanwhile, the relative intensity of the $\mathrm{OH}^{-}$and $\mathrm{HPO}_{4}{ }^{2-}$ signals at 0.2 ppm and 11 ppm, respectively, decreases. Equilibrium of the magnetization between HAp and Pluronic polymer evidenced by similar relative peak intensities occurs in all ${ }^{1} \mathrm{H}$ cross sections within $500 \mathrm{~ms}{ }^{1} \mathrm{H}$ spin diffusion in all samples. ${ }^{1} \mathrm{H}$ spectra taken at $1 \mathrm{ppm}{ }^{31} \mathrm{P}$ exhibit faster spin diffusion to polymer protons than those taken at $2.8 \mathrm{ppm}$, which again confirms that the disordered phosphate component is at the organic-inorganic interface.

Nanocrystal size. The size of the nanocrystals is crucial to the overall performance and properties of nanocomposite materials. In the present syntheses, it is also a measure of citrate's specific molecular interactions with the mineral phase, whereby it promotes, and more importantly, stabilizes the HAp phase at a few nanometer thickness. The rate of ${ }^{1} \mathrm{H}$ spin diffusion from the organic protons to the mineral matrix, observed in a series of ${ }^{1} \mathrm{H}_{-}{ }^{31} \mathrm{P}$ HetCor spectra reveals that spin diffusion between the organic and inorganic protons is faster 
as the citrate concentration is increased from $0 \mathrm{mM}$ to $40 \mathrm{mM}$. This indicates that on average, the mineral has a larger surface area in contact with the organic phase in the $40 \mathrm{mM}$ citrate sample as compared to the lower citrate concentrations; alternatively, a steady decrease in the average crystal size as more citrate is added. This trend is seen most clearly in a comparison of spectra from all samples after $5 \mathrm{~ms}$ of ${ }^{1} \mathrm{H}$ spin diffusion (Figure 3.10). With the intensities of the $\mathrm{OH}^{-}$peaks scaled to equal height, the spin diffusion rate can be gauged by the intensity of the polymer ${ }^{1} \mathrm{H}$ peaks, in particular the $\mathrm{OCH}_{2} / \mathrm{OCH}$ at $4 \mathrm{ppm}$. The sample with $40 \mathrm{mM}$ citrate shows the most intense $\mathrm{OCH}_{2} / \mathrm{OCH}$ peak and thus the fastest ${ }^{1} \mathrm{H}$ spin diffusion and therefore the smallest HAp crystals, followed by the samples with 10,2 , and then $0 \mathrm{mM}$ citrate. The faster spin diffusion may also imply a more extensive interaction between the mineral and the polymer matrix, which is important to the stability of the final nanocomposite.

The 2D HetCor experiments with spin-diffusion provide a direct comparative but qualitative measurement of the nanocrystal size. More quantitative measurements of the surface-to-volume ratio of the mineralized HAp nanocrystals in these samples were carried out using ${ }^{1} \mathrm{H}_{-}{ }^{31} \mathrm{P}$ HARDSHIP experiments. In these experiments, the ${ }^{31} \mathrm{P}$ magnetization is dephased (destroyed) in a selective manner mostly by polymer and surface protons (Figure 3.11). The faster the measured dephasing, the larger the surface-to-volume ratio and the smaller the crystals. The measurement shows that increasing the citrate concentration decreases the synthetic HAp crystal size within the polymer matrix. The dephasing behavior of the sample with $2 \mathrm{mM}$ citrate is similar to that of avian bone. This is compatible with the average citrate concentration of $\sim 2-3 \mathrm{mM}$ in body fluid and tissues in chicken ${ }^{39}$. The HARDSHIP dephasing of bovine bone is only slightly faster than that of synthetic HAp made with $0 \mathrm{mM}$ citrate, consistent with a citrate concentration of $\sim 0.078 \mathrm{mM}$ in bovine blood ${ }^{40}$.

The crystal size, particularly the thickness for elongated structures, of HAp can be estimated from the results of ${ }^{1} \mathrm{H}_{-}{ }^{31} \mathrm{P}$ HetCor experiments with ${ }^{1} \mathrm{H}$ spin diffusion, the surface and core fractions derived from deconvoluted DP spectra, the width of the (310) peak in Xray diffraction, and ${ }^{31} \mathrm{P}\left\{{ }^{1} \mathrm{H}\right\}$ HARDSHIP signal decay. In principle, the ${ }^{1} \mathrm{H}$ spin diffusion time required to reach equilibrium in ${ }^{1} \mathrm{H}_{-}{ }^{31} \mathrm{P}$ HetCor experiments can be used to estimate the thickness of the inorganic domain, but this requires knowledge of spin diffusion coefficients 
and ${ }^{1} \mathrm{H}$ densities in the organic and inorganic layers. Nevertheless, even without these parameters available, the thickness of apatite crystals can still be approximately evaluated based on the similar synthetic systems in our previous published results. All the samples discussed in this paper exhibit faster spin diffusion than the Pluronic30-8 sample ${ }^{16}$ made with Pluronic polymer and calcium phosphate salt without citrate under similar experimental conditions, which was characterized by various techniques, including wide-angle X-ray diffraction, solid state NMR, small angle neutron/X-ray scattering (SANS/SAXS), and TEM, and in which the thickness of the HAp crystals is $\sim 4.8 \mathrm{~nm}$. Therefore, the thickness of the HAp crystals in all the samples in this paper is $<5 \mathrm{~nm}$.

With a surface phosphate layer of $\mathrm{d}_{\mathrm{s}} \approx 0.4-\mathrm{nm}$ thickness, the typical aspect ratio of crystals in bovine bone ${ }^{41,42}$ and the surface-to-volume ratio of apatite crystals, the crystallite thickness $d$ of the four synthetic samples presented in this paper can be extrapolated based on Fraction of surface $\mathrm{HPO}_{4}{ }^{2-}$ and $\mathrm{PO}_{4}{ }^{3-}=\mathrm{d}_{\mathrm{s}} \times($ Surface/Volume $)$

$$
=\mathrm{d}_{\mathrm{s}} \times 2 \times(\mathrm{L} \times \mathrm{W}+\mathrm{L} \times \mathrm{d}+\mathrm{W} \times \mathrm{d}) /(\mathrm{L} \times \mathrm{W} \times \mathrm{d})
$$

For long thin crystals, Eq.1 simplifies to $\mathrm{S} / \mathrm{V} \sim 2 /$ d. Since the length (L) and width (W) of the crystals are not infinite, a more accurate estimate needs to consider changes in length and width of HAp crystals. Assuming a fixed aspect ratio of $\mathrm{d}: \mathrm{W}: \mathrm{L}=1: 3: 6, \mathrm{~S} / \mathrm{V}=3 / \mathrm{d}$ according to Eq. 3.1 and the crystal thicknesses of the synthetic HAp range between 2.3 and $4.4 \mathrm{~nm}$, as listed in Table 3.1.

For comparison, the HAp crystal diameters have also been estimated by applying the DebyeScherrer equation

$$
\mathrm{d}=\mathrm{K} \lambda /\left(\mathrm{B}_{1 / 2} \cos \theta\right)
$$

to the X-ray diffraction peak (310) of the washed samples synthesized with different citrate concentrations, where $K$ is chosen as $0.9, \lambda=0.154 \mathrm{~nm}$ is the $\mathrm{x}$-ray wavelength, $B_{1 / 2}$ is the full width at half maximum (FWHM) of the diffraction peak, and $\theta=20^{\circ}$ is the diffraction angle of the (310) peak. The crystal thicknesses calculated from X-ray diffraction, see Table 3.1, are somewhat smaller than those estimated from the phosphate compositions. This can 
be attributed to the disordered surface layers (>0.6 nm thickness combined) that may not contribute to the coherent scattering.

Analysis of ${ }^{31} \mathrm{P}\left\{{ }^{1} \mathrm{H}\right\}$ HARDSHIP NMR data by curve fitting ${ }^{34}$ as shown in Figure 3.11 gives crystal thicknesses, again for $\mathrm{d}: \mathrm{W}: \mathrm{L}=1: 3: 6$ and with $2 \times 0.3 \mathrm{~nm}$ of surface layers beyond the surface phosphorus layers. The ${ }^{1} \mathrm{H}$ transverse relaxation time in the simulations was $\mathrm{T}_{2}=0.6 \mathrm{~ms}$, and the ${ }^{1} \mathrm{H}$ density of the polymer phase, $1 /(0.29 \mathrm{~nm})^{3}$. The values listed in Table 3.1 are only slightly smaller than those from the surface-top-volume ratio.

\section{Mechanism of citrate controlling the apatite crystal size}

The three carboxyl groups of citrate have $\mathrm{pK}_{\mathrm{a}}$ values of 3.1, 4.8, and 6.4, therefore, at $\mathrm{pH}=7.5-8$, they are completely ionized and can bind strongly to $\mathrm{Ca}^{2+}$ on the HAp surface. In studies of citrate in natural bone $\mathrm{e}^{23}$ or in vitro research on citrate interacting with

HAp $^{26,27,29,43}$, citrate always exists on the surface of apatite crystals without being incorporated within the crystal lattice, due to the relatively larger size of citrate molecules compared with lattice ions, such as phosphate or $\mathrm{OH}^{-}$; this indicates that once citrate molecule occupies certain surface area of growing apatite, the further crystal growth on top of that area will be terminated unless the citrate molecule departs. In addition, surface bound citrate molecules generate a negatively charged surface area ${ }^{26}$, which repels negatively charged phosphate ions, thus inhibiting further crystal growth. The negatively charged apatite surface also forbids the aggregation of the primary crystallites into bigger particles via electrostatic repulsion ${ }^{26}$.

\section{Conclusions}

The molecular species, their relative concentrations, and the proximity of different inorganic and organic components in synthesized bone-mimicking nanocomposites have been assessed by a full complement of microscopic, diffraction, and spectroscopic techniques. These methodologies show that the addition of citrate in the bottom-up synthesis of self-assembled Pluronic polymer and HAp nanocomposites enables molecular control over the growth of the apatite crystal size in a manner that closely mimics native bone. The combination of XRD and ${ }^{1} \mathrm{H}-{ }^{31} \mathrm{P}$ NMR techniques show that citrate is able to stabilize HAp 
crystals of extremely small thickness ( $2 \mathrm{~nm}, \sim 3$ unit cells), over other calcium phosphate species. The mineralization of the organic matrix occurs without disturbing the supramolecular structure of the polymer gel, as documented by the nearly unchanged SANS patterns with and without citrate. The crystal size of HAp can be fine tuned on the nanometer scale by varying the citrate concentration. By using similar concentrations of citrate as in body fluid, we have successfully reproduced apatite nanocrystals of similar sizes and morphologies as in natural bone. The increase in citrate concentrations to $40 \mathrm{mM}$ makes the $\mathrm{HPO}_{4}{ }^{2-} / \mathrm{PO}_{4}-\mathrm{H}_{2} \mathrm{O}$ surface species the dominant fraction (at $40 \mathrm{mM}$ citrate) of the mineral phase, as determined by careful analysis of quantitative ${ }^{31} \mathrm{P}$ NMR spectra. The presence of citrate and the structural similarity of the synthetic HAp nanocrystals with bone apatite may contribute to better biocompatibility of these nanocomposites.

Acknowledgement. This work was supported by the U.S. Department of Energy, Office of Basic Energy Science, Division of Materials Sciences and Engineering. The research was performed at the Ames Laboratory. Ames Laboratory is operated for the U.S. Department of Energy by Iowa State University under Contract No. DE-AC02-07CH11358. Thanks to Dr. Jennifer O'Donnell (Iowa State University) for helping to perform the SANS experiments.

\section{References}

1. Buckwalter, J. A.; Glimcher, M. J.; Cooper, R. R.; Recker, R., J Bone Joint Surg Am 1995, 77, 1256.

2. Rey, C.; Combes, C.; Drouet, C.; Glimcher, M. J., Osteoporos Int 2009, 20, 2155.

3. Weiner, S.; Traub, W., FASEB J. 1992, 6, 879.

4. Weiner, S.; Wagner, H. D., Annu. Rev. Mater. Sci. 1998, 28, 271.

5. Zhu, W.; Robey, P. G.; Boskey, A. L., The regulatory role of matrix proteins in mineralization of bone. In Osteoporosis, R., M.; D., F.; D.A., N., Eds. Elsevier Academic Press: San Diego, CA, 2008; Vol. 1.

6. Wasserman, N.; Brydges, B.; Searles, S.; Akkus, O., Bone 2008, 43, 856. 
7. Palmer, L. C.; Newcomb, C. J.; Kaltz, S. R.; Spoerke, E. D.; Stupp, S. I., Chem. Rev. 2008, 108, 4754.

8. Salgado, A. J.; Coutinho, O. P.; Reis, R. L., Macromol. Biosci. 2004, 4, 743.

9. Heath, C. A., Trends Biotechnol. 2000, 18, 17.

10. Landis, W. J.; Silver, F. H.; Freeman, J. W., J. Mater. Chem. 2006, 16, 1495.

11. Cui, F. Z.; Li, Y.; Ge, J., Mater. Sci. Eng., R 2007, 57, 1.

12. Shin, H.; Jo, S.; Mikos, A. G., Biomaterials 2003, 24, 4353.

13. Vallet-Regi, M., C. R. Chim. 2010, 13, 174.

14. Enlow, D.; Rawal, A.; Kanapathipillai, M.; Schmidt-Rohr, K.; Mallapragada, S.; Lo, C. T.; Thiyagarajan, P.; Akinc, M., J. Mater. Chem. 2007, 17, 1570.

15. Kanapathipillai, M.; Yusufoglu, Y.; Rawal, A.; Hu, Y. Y.; Lo, C. T.; Thiyaigarajan, P.; Kalay, Y. E.; Akinc, M.; Mallapragada, S.; Schmidt-Rohr, K., Chem. Mater. 2008, $20,5922$.

16. Yusufoglu, Y.; Hu, Y.; Kanapathipillai, M.; Kramer, M.; Kalay, Y. E.; Thiyagarajan, P.; Akinc, M.; Schmidt-Rohr, K.; Mallapragada, S., J. Mater. Res. 2008, 23, 3196.

17. Hu, Y. Y.; Yusufoglu, Y.; Kanapathipillai, M.; Yang, C. Y.; Wu, Y. Q.;

Thiyagarajan, P.; Deming, T.; Akinc, M.; Schmidt-Rohr, K.; Mallapragada, S., Soft Matter 2009, 5, 4311.

18. Pramanik, S.; Kar, K. In Apatite-poly(ether etherketone)nano composites:prosthesis materials: SBF-conditioned study, Annual Technical Conference-Society of Plastics Engineers, 2007; Society of Plastics Engineers: 2007; pp 2558.

19. Song, J.; Malathong, V.; Bertozzi, C. R., J. Am. Chem. Soc. 2005, 127, 3366.

20. Armentano, I.; Dottori, M.; Fortunati, E.; Mattioli, S.; Kenny, J. M., Polym. Degrad. Stab. 2010, $95,2126$.

21. Gao, H. J.; Ji, B. H.; Jager, I. L.; Arzt, E.; Fratzl, P., Proc. Natl. Acad. Sci. U. S. A. 2003, 100, 5597.

22. Gay, S.; Arostegui, S.; Lemaitre, J., Mater. Sci. Eng. C 2009, 29, 172.

23. Hoang, Q. Q.; Sicheri, F.; Howard, A. J.; Yang, D. S. C., Nature 2003, 425, 977.

24. Hu, Y. Y.; Rawal, A.; Schmidt-Rohr, K., Proc. Natl. Acad. Sci. U. S. A. 2010, 107, 22425. 
25. Sato, K.; Kumagai, Y.; Tanaka, T., J. Biomed. Mater. Res. 2000, 50, 16.

26. Martins, M. A.; Santos, C.; Almeida, M. M.; Costa, M. E. V., J. Colloid Interface Sci. 2008, 318, 210.

27. Lopez-Macipe, A.; Gomez-Morales, J.; Rodriguez-Clemente, R., Adv. Mater. 1998, $10,49$.

28. Jiang, W. G.; Chu, X. B.; Wang, B.; Pan, H. H.; Xu, X. R.; Tang, R. K., J. Phys. Chem. B 2009, 113, 10838.

29. Hempel, U.; Reinstorf, A.; Poppe, M.; Fischer, U.; Gelinsky, M.; Pompe, W.;

Wenzel, K. W., J Biomed Mater Res B Appl Biomate 2004, 71B, 130.

30. Schneiders, W.; Reinstorf, A.; Pompe, W.; Grass, R.; Biewener, A.; Holch, M.;

Zwipp, H.; Rammelt, S., Bone 2007, 40, 1048.

31. Burton, A. W., J. Am. Chem. Soc. 2007, 129, 7627.

32. Taguchi, A.; Schuth, F., Microporous Mesoporous Mater. 2005, 77, 1.

33. Reisfeld, R.; Saraidarov, T., Opt. Mater. 2006, 28, 64.

34. Schmidt-Rohr, K.; Rawal, A.; Fang, X. W., J. Chem. Phys. 2007, 126, 054701/1.

35. Hou, S. S.; Beyer, F. L.; Schmidt-Rohr, K., Solid State Nucl. Magn. Reson. 2002, 22, 110.

36. Liao, S.; Watari, F.; Uo, M.; Ohkawa, S.; Tamura, K.; Wang, W.; Cui, F. Z., J Biomed Mater Res B Appl Biomate 2005, 74B, 817.

37. Muller, S. A.; Engel, A., Chimia 2006, 60, A749.

38. Sousa, A. A.; Hohmann-Marriott, M.; Aronova, M. A.; Zhang, G.; Leapman, R. D., J. Struct. Biol. 2008, 162, 14.

39. Hertelendy, F.; Taylor, T. G., J Bone Joint Surg Br 1963, 45, 220.

40. Thunberg, T., Physiol. Rev. 1953, 33, 1.

41. Fratzl, P.; Schreiber, S.; Klaushofer, K., Connect Tissue Res 1996, 35, 9.

42. Tong, W.; Glimcher, M. J.; Katz, J. L.; Kuhn, L.; Eppell, S. J., Calcif. Tissue Int. 2003, 72, 592.

43. Rhee, S. H.; Tanaka, J., Biomaterials 1999, 20, 2155. 


\section{Tables and Figures}

Table 3.1. Composition of phosphates formed in Pluronic polymer and HAp nanocomposites with different citrate concentrations quantified based on the deconvolution of the DP spectra. The estimated HAp crystal thicknesses based on the ratio of surface phosphates $\left(\mathrm{HPO}_{4}{ }^{2-}\right.$ and $\left.\mathrm{PO}_{4}{ }^{3-}-\mathrm{H}_{2} \mathrm{O}\right)$ and the total phosphates are also listed and compared with the thicknesses calculated by applying the Debye-Scherrer formula to the (310) peak in the wide-angle X-ray diffraction patterns of the washed samples.

\begin{tabular}{|c|c|c|c|c|c|c|}
\hline $\begin{array}{c}\text { Citrate } \\
\text { Concentration }\end{array}$ & $\begin{array}{l}\text { Core } \\
\mathrm{PO}_{4}{ }^{3-}\end{array}$ & $\begin{array}{c}\text { Surface } \\
\mathrm{HPO}_{4}{ }^{2-} \\
\text { and } \\
\mathrm{PO}_{4}{ }^{3-} \\
\mathrm{H}_{2} \mathrm{O}\end{array}$ & $\begin{array}{c}\text { Estimated } \\
\text { crystal } \\
\text { thickness } \\
\text { from surface } \\
\text { fraction } \\
( \pm 0.5 \mathrm{~nm})\end{array}$ & $\begin{array}{c}\text { Estimated } \\
\text { crystal } \\
\text { thickness } \\
\text { HARDSHIP } \\
\text { NMR } \\
( \pm 20 \%)\end{array}$ & $\begin{array}{c}\mathrm{B}_{1 / 2} \text { (radian) } \\
\text { of ( } 310) \\
\text { peaks from } \\
\text { XRD of } \\
\text { washed } \\
\text { samples }\end{array}$ & $\begin{array}{c}\text { Estimated } \\
\text { crystal } \\
\text { diameter } \\
\text { from X-ray } \\
\text { diffraction }\end{array}$ \\
\hline $0 \mathrm{mM}$ & $73 \%$ & $27 \%$ & $4.4 \mathrm{~nm}$ & $3.5 \mathrm{~nm}$ & $0.03(7 \pm 5)$ & $4.0 \mathrm{~nm}$ \\
\hline $2 \mathrm{mM}$ & $59 \%$ & $41 \%$ & $2.9 \mathrm{~nm}$ & $2.7 \mathrm{~nm}$ & $0.06(1 \pm 7)$ & $2.4 \mathrm{~nm}$ \\
\hline $10 \mathrm{mM}$ & $57 \%$ & $43 \%$ & $2.8 \mathrm{~nm}$ & $2.6 \mathrm{~nm}$ & $0.06(3 \pm 7)$ & $2.3 \mathrm{~nm}$ \\
\hline $40 \mathrm{mM}$ & $48 \%$ & $52 \%$ & $2.3 \mathrm{~nm}$ & $2.2 \mathrm{~nm}$ & $0.1(0 \pm 2)$ & $1.5 \mathrm{~nm}$ \\
\hline
\end{tabular}




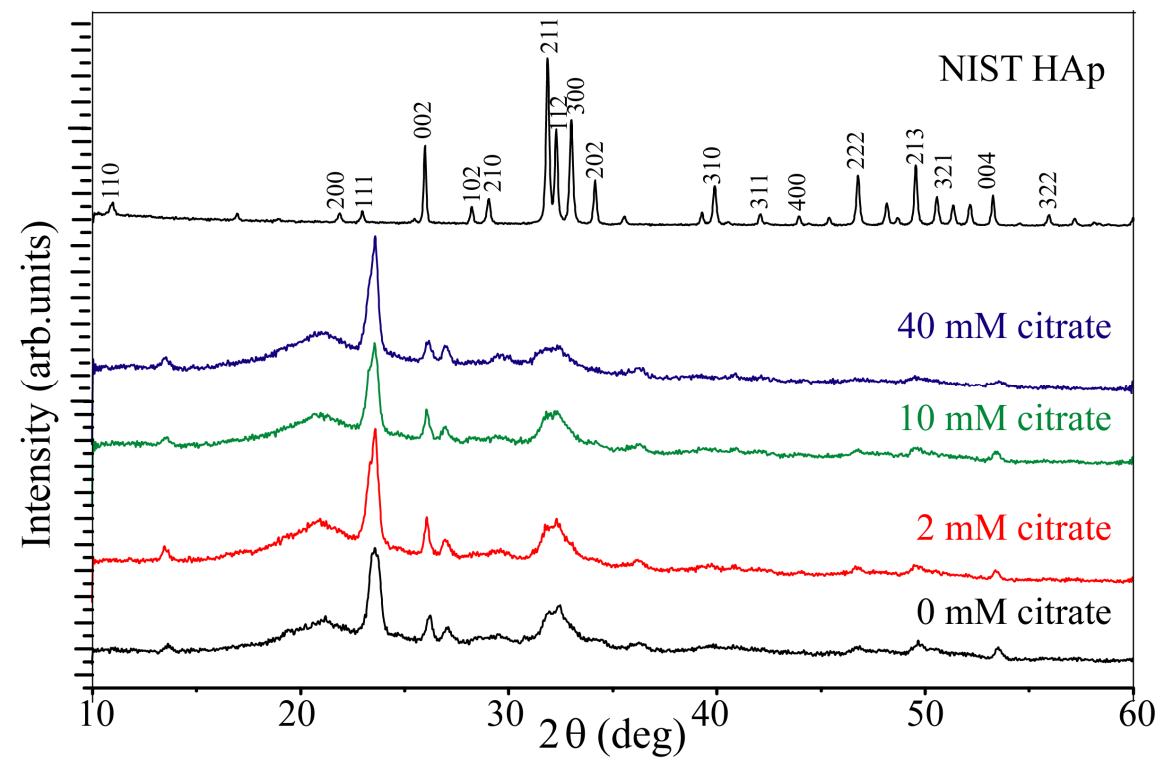

Figure 3.1. XRD patterns of Pluronic polymer-hydroxyapaite nanocomposite samples with different citrate concentrations after freeze drying. The diffraction pattern of NIST HAp is shown for reference. Data were collected by X. Liu.

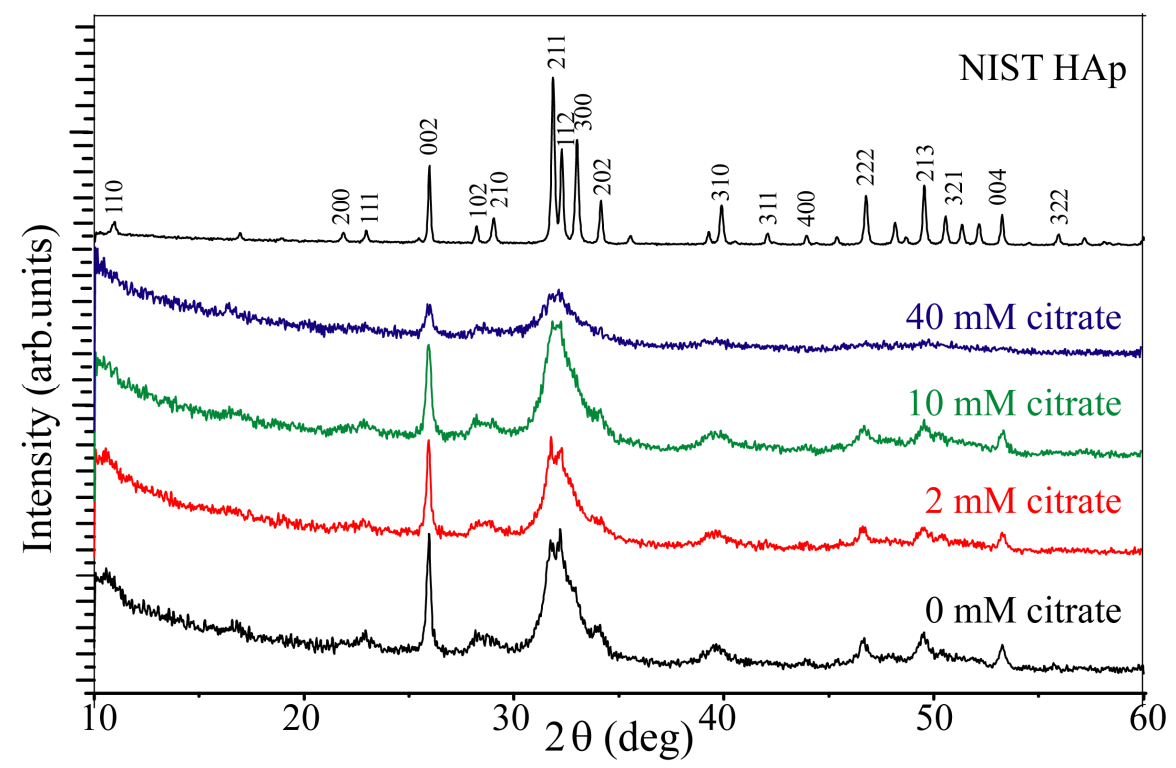

Figure 3.2. XRD patterns of Pluronic polymer-hydroxyapatite nanocomposites after washing. The diffraction pattern of NIST HAp is shown for reference. Data were collected by X. Liu. 



Figure 3.3. HAADF-STEM images of hydroxyapatite nanocrystals fabricated within a Pluronic polymer matrix using different citrate concentrations: (a) $0 \mathrm{mM}$; (b) $2 \mathrm{mM}$; (c) 10 mM; (d) $40 \mathrm{mM}$. All scale bars are $50 \mathrm{~nm}$. Data were collected by T. Prozorov. 

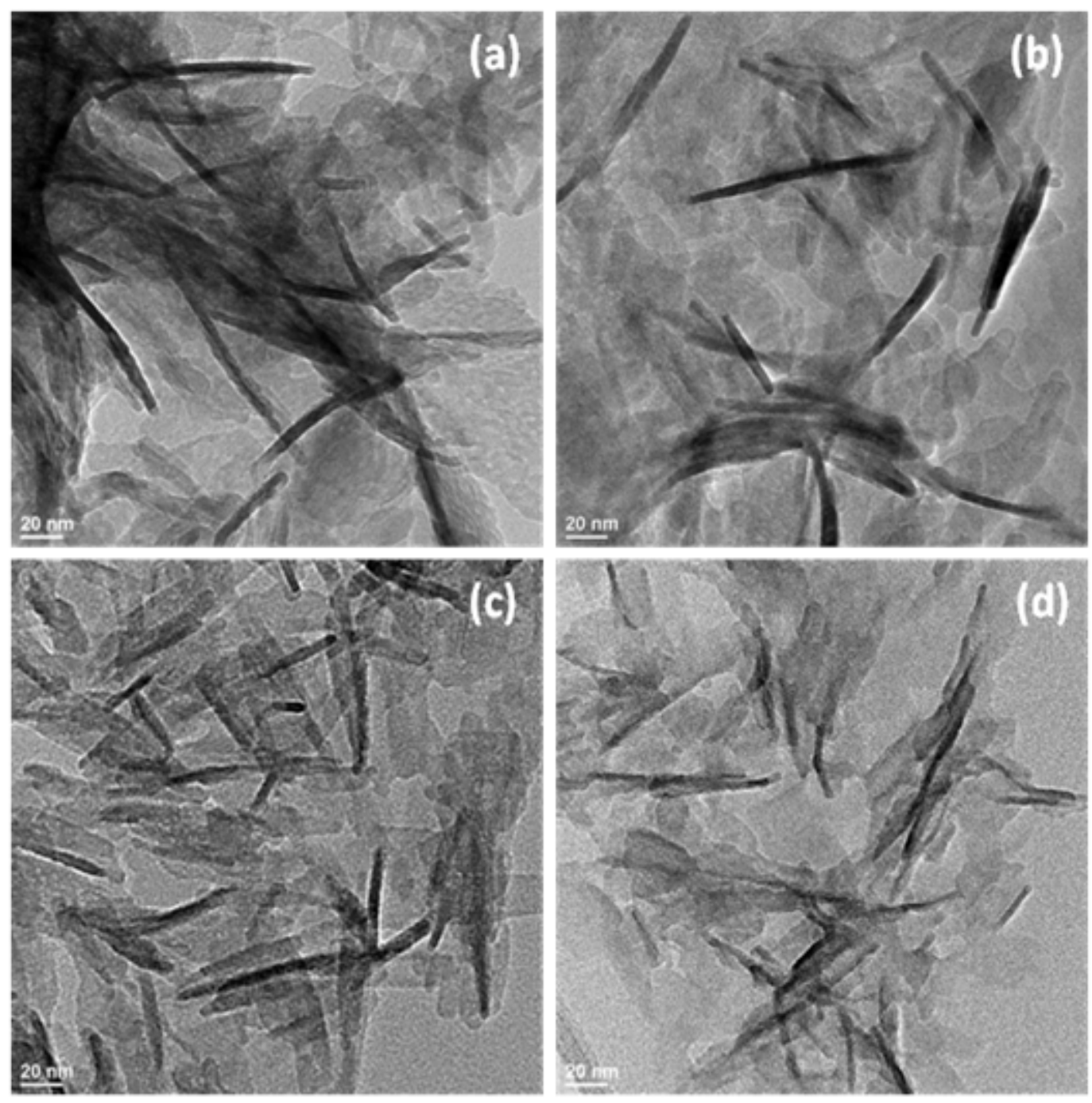

Figure 3.4. Bright field TEM images of hydroxyapatite nanocrystals fabricated within a Pluronic polymer matrix using different citrate concentrations: (a) $0 \mathrm{mM}$; (b) $2 \mathrm{mM}$; (c) 10 $\mathrm{mM}$; (d) $40 \mathrm{mM}$. The magnification is 880000 , and scale bars are $20 \mathrm{~nm}$. Data were obtained by Yusuf Yusufgolu. Data were collected by T. Prozorov. 


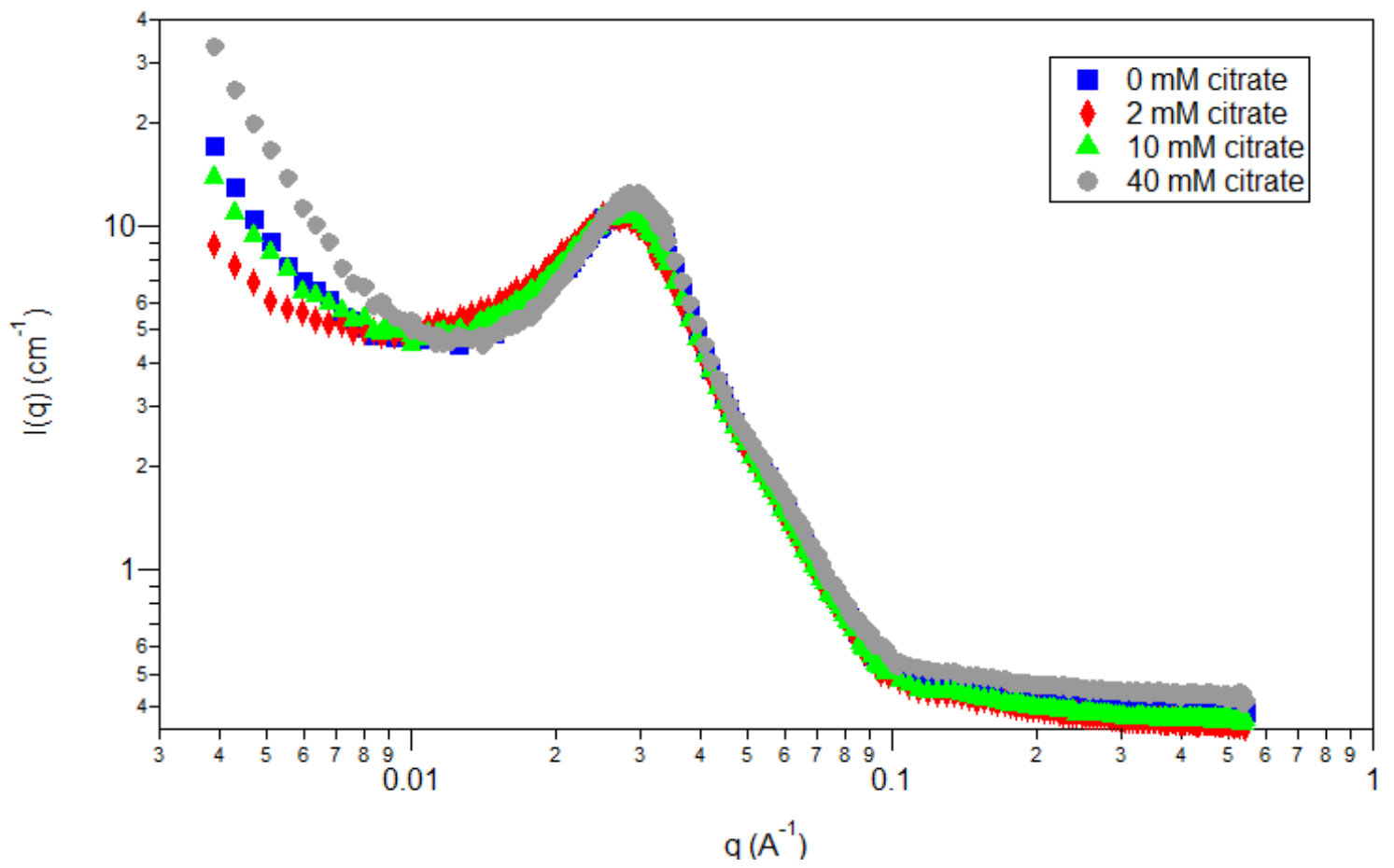

Figure 3.5. SANS curves of Pluronic-hydroxyapatite nanocomposites fabricated using different citrate concentrations. Data were collected by X. Liu. 

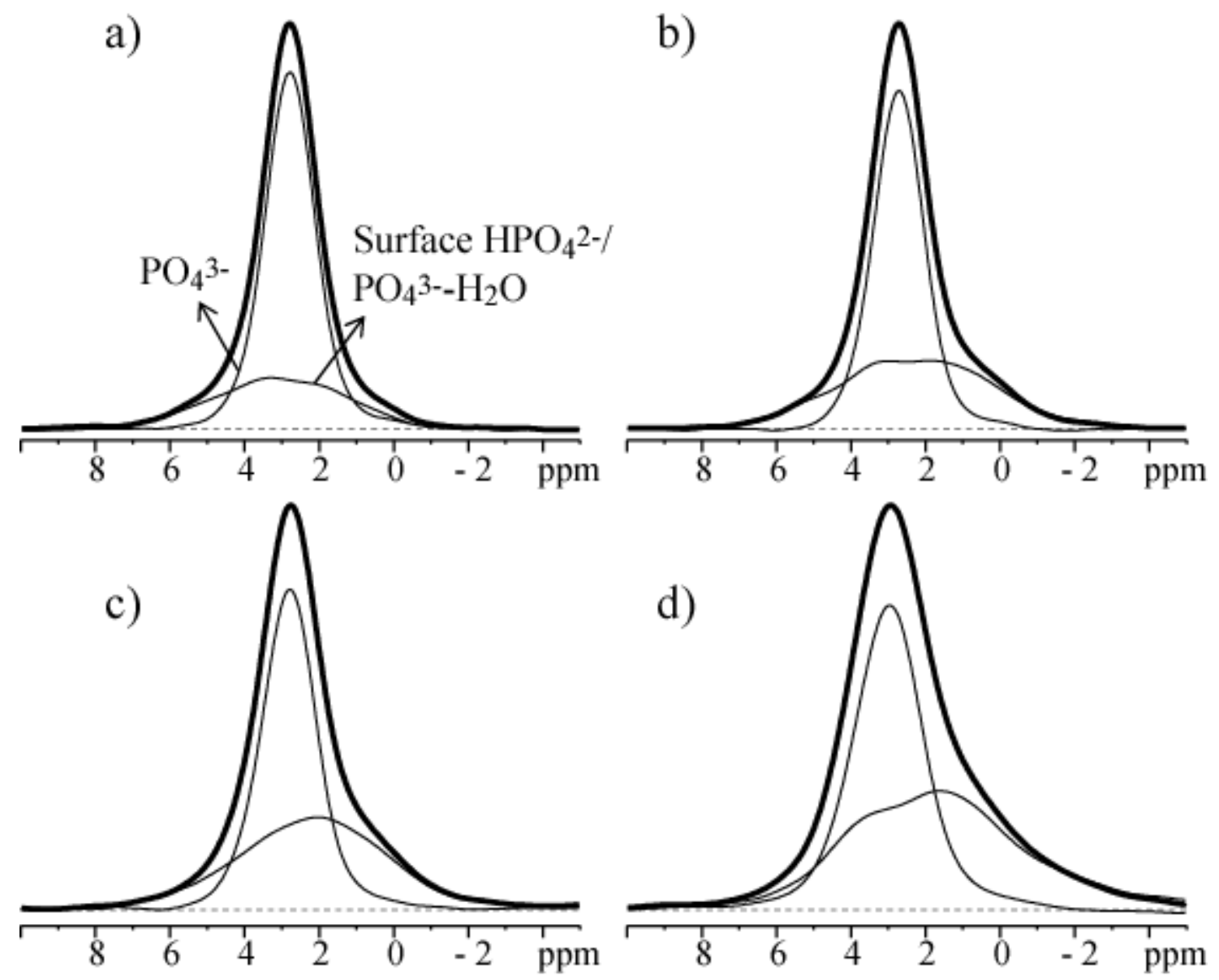

Figure 3.6. Direct polarization (DP) ${ }^{31} \mathrm{P}$ NMR spectra of composites synthesized with Pluronic polymer, calcium phosphate, and with ammonium citrate of different concentration. a) $0 \mathrm{mM}$ citrate, b) $2 \mathrm{mM}$ citrate, c) $10 \mathrm{mM}$ citrate, d) $40 \mathrm{mM}$ citrate. The spectra have been deconvoluted into a broad component (surface $\mathrm{PO}_{4}{ }^{3-} / \mathrm{PO}_{4}{ }^{3-}-\mathrm{H}_{2} \mathrm{O}$ ) and a relative sharp peak from bulk crystalline hydroxyapatite (HAp) based on their different HARDSHIP dephasing behaviors by polymer and surface protons. Magic angle spinning frequency $v_{\mathrm{r}}=6.5 \mathrm{kHz}$. 


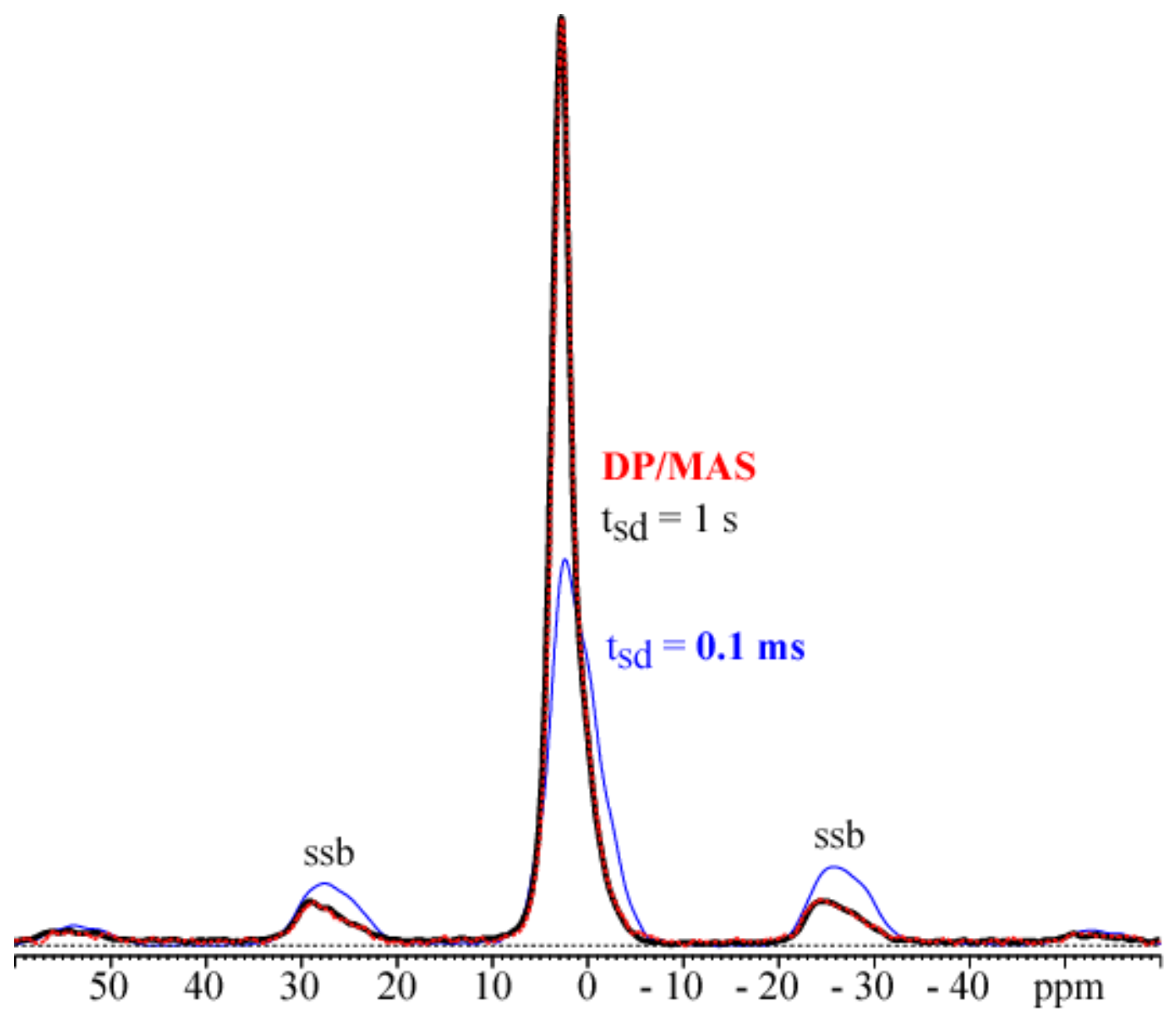

Figure 3.7. ${ }^{31} \mathrm{P}$ NMR spectra of the Pluronic polymer- HAp composite with $40 \mathrm{mM}$ citrate. Cross polarization $(\mathrm{CP}){ }^{31} \mathrm{P}$ NMR spectra with short contact time of $0.1 \mathrm{~ms}$ and ${ }^{31} \mathrm{P}$ spin diffusion time of $0.1 \mathrm{~ms}$ (thick line) and $1 \mathrm{~s}$ (thin line) are compared. The direct-polarization spectrum (dashed line, nearly indistinguishable from the 1-s spectrum) is used as a reference to gauge the completion of spin diffusion. Spinning sidebands are labeled as "ssb". $v_{\mathrm{r}}=4.3$ kHz. 


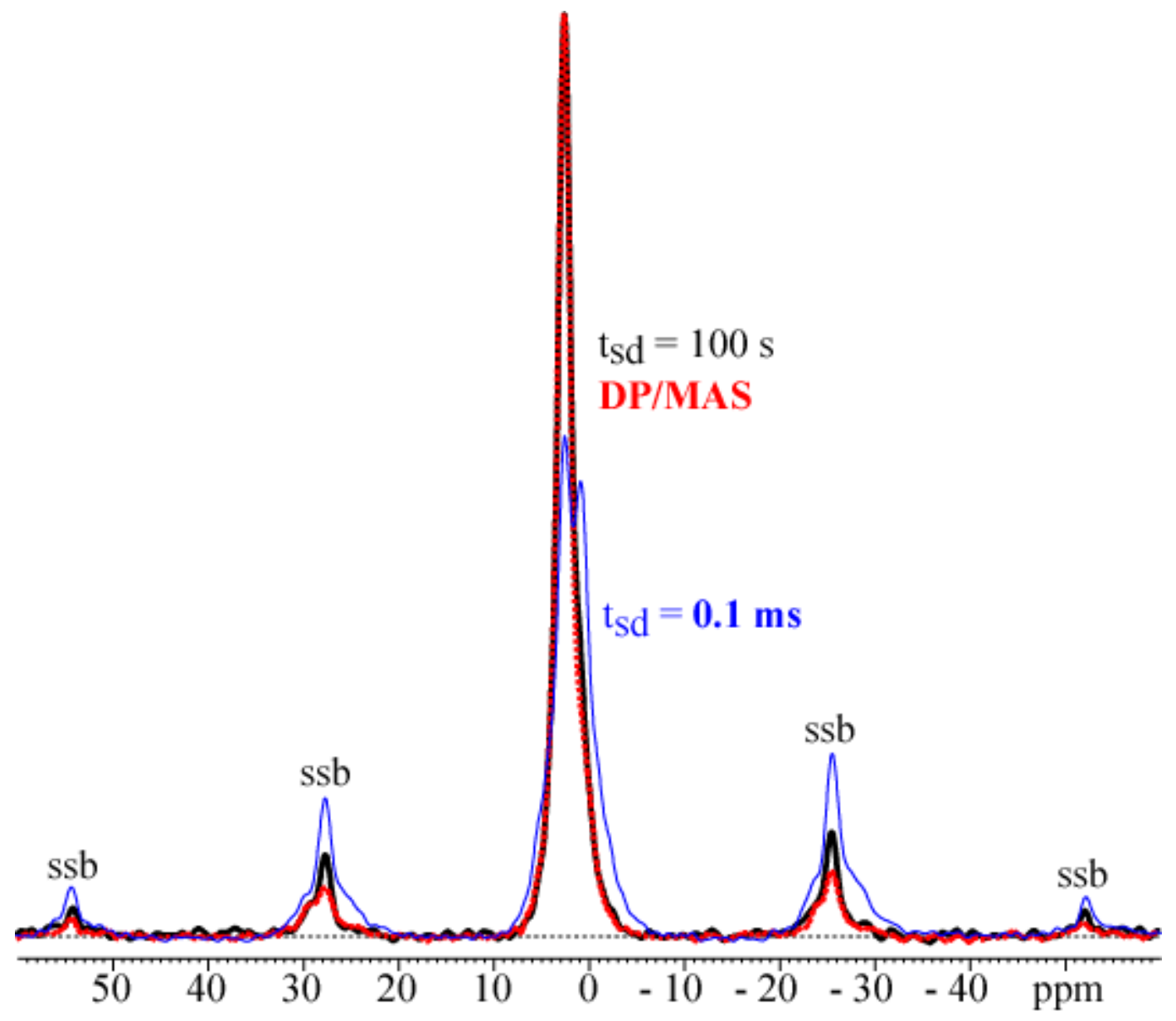

Figure 3.8. ${ }^{31} \mathrm{P}$ NMR spectra of a Pluronic polymer and calcium phosphate composite with 0 $\mathrm{mM}$ citrate. Cross polarization $(\mathrm{CP}){ }^{31} \mathrm{P}$ NMR spectra with short contact time of $0.1 \mathrm{~ms}$ and ${ }^{31} \mathrm{P}$ spin diffusion times of $0.1 \mathrm{~ms}$ (thick line) and $100 \mathrm{~s}$ (thin line) are compared. The directpolarization spectrum (dashed line) is used as a reference to gauge the completion of spin diffusion. Spinning sidebands are labeled as "ssb". $v_{\mathrm{r}}=4.3 \mathrm{kHz}$. 

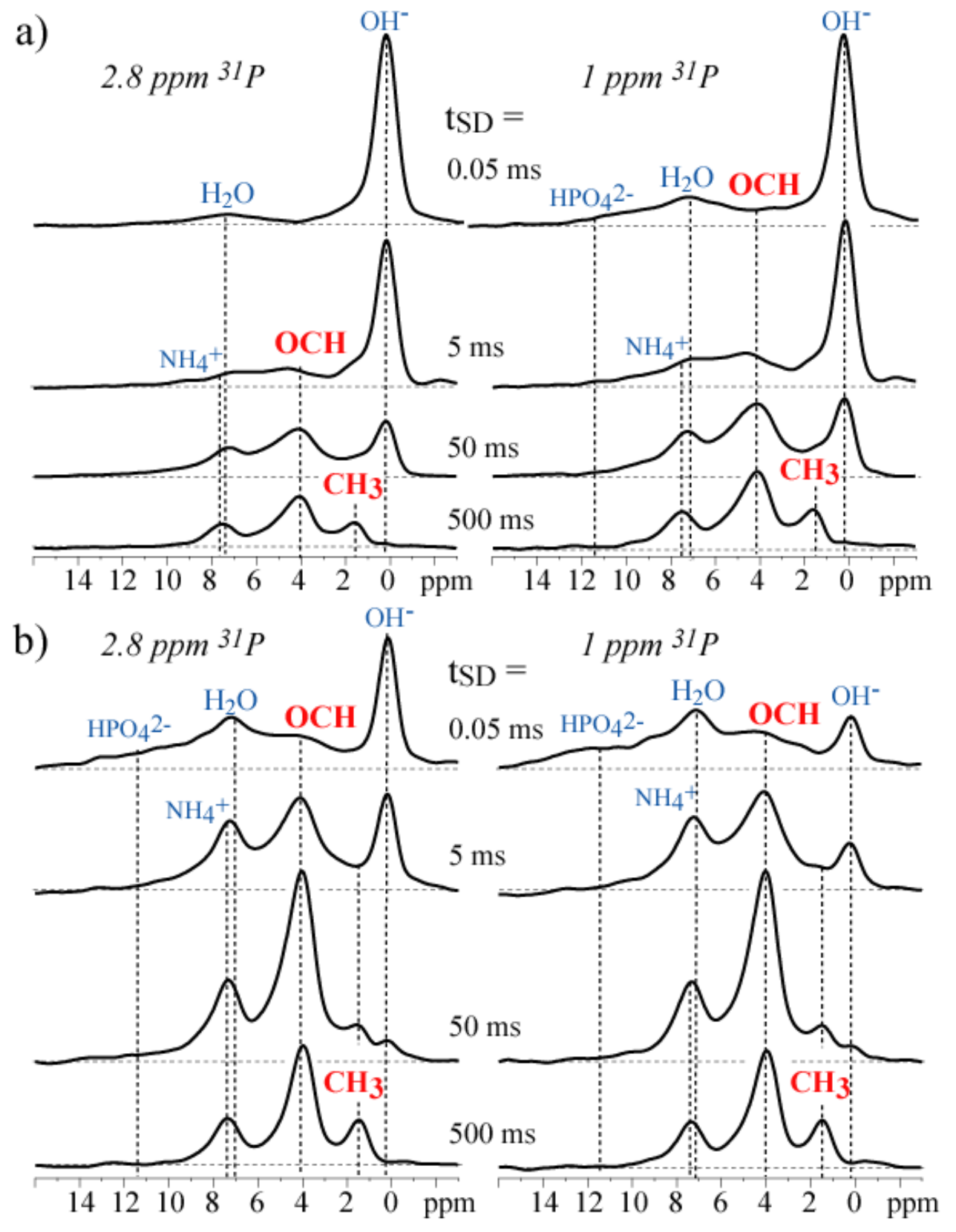

Figure 3.9. ${ }^{1} \mathrm{H}$ spectra from cross sections taken at $2.8 \mathrm{ppm}$ and $1.0 \mathrm{ppm}{ }^{31} \mathrm{P}$ of $2 \mathrm{D}{ }^{1} \mathrm{H}-{ }^{31} \mathrm{P}$ HetCor NMR spectra of composites synthesized with Pluronic polymer, calcium phosphate, and a) $0 \mathrm{mM}$, and b) $40 \mathrm{mM}$ ammonium citrate. The ${ }^{1} \mathrm{H}$ spin diffusion times $\mathrm{t}_{\mathrm{SD}}$ are $0.05,5$, 50 , and $500 \mathrm{~ms}$. 


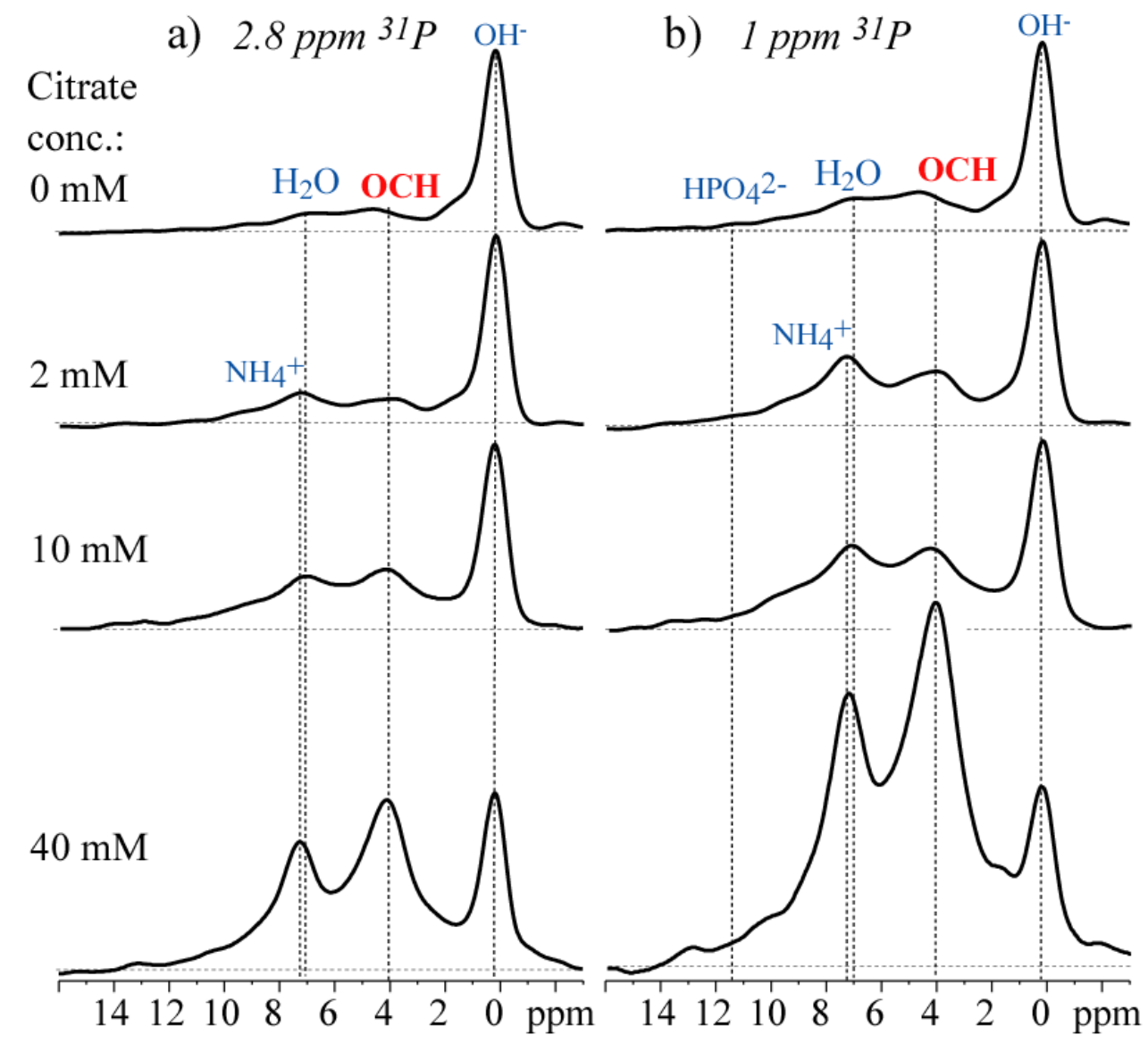

Figure 3.10. ${ }^{1} \mathrm{H}$ spectra from cross sections of $2 \mathrm{D}{ }^{1} \mathrm{H}_{-}{ }^{31} \mathrm{P}$ HetCor NMR spectra with $5 \mathrm{~ms}$ of ${ }^{1} \mathrm{H}$ spin diffusion of Pluronic polymer, calcium phosphate, and 0, 2, 10, or $40 \mathrm{mM}$ ammonium citrate composite. ${ }^{1} \mathrm{H}$ spectra were taken at a) $2.8 \mathrm{ppm}{ }^{31} \mathrm{P}$, b) $1.0 \mathrm{ppm}{ }^{31} \mathrm{P}$. The height of the $\mathrm{OH}^{-}$peak at $0.2 \mathrm{ppm}$ has been normalized for convenience of comparison. 


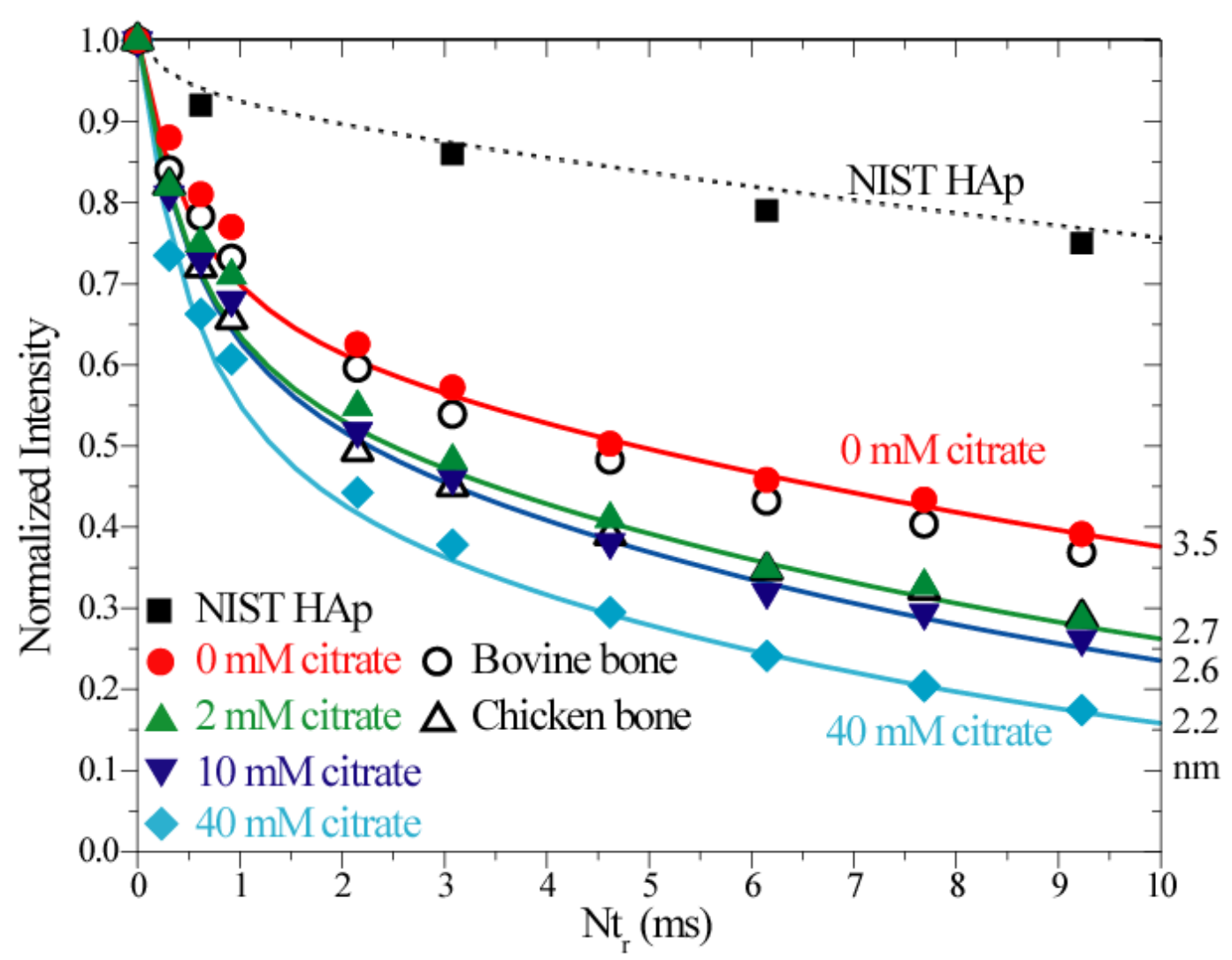

Figure 3.11. ${ }^{31} \mathrm{P}\left\{{ }^{1} \mathrm{H}\right\}$ HARDSHIP dephasing in composites of Pluronic polymer, calcium phosphate, and ammonium citrate of $0,2,10$, and $40 \mathrm{mM}$ concentrations. NIST HAp is used as a reference of the decay only due to protons within the crystal lattice. Bovine bone and chicken bone are used to compare the crystal size of synthetic HAp and apatite in bones. Solid lines are simulated dephasing curves to obtain the thicknesses of crystals. The simulation procedure is detailed in reference [34]. 


\section{Supporting Information for}

Biomimetic Self-assembling Nanocomposites of Block Copolymer and Hydroxyapatite with Nanocrystal Size Controlled by Citrate

Y.-Y. Hu, X. Liu, X. Ma, A. Rawal, T. Prozorov, M. Akinc, S. K. Mallapragada, K. Schmidt-

$$
\text { Rohr* }
$$

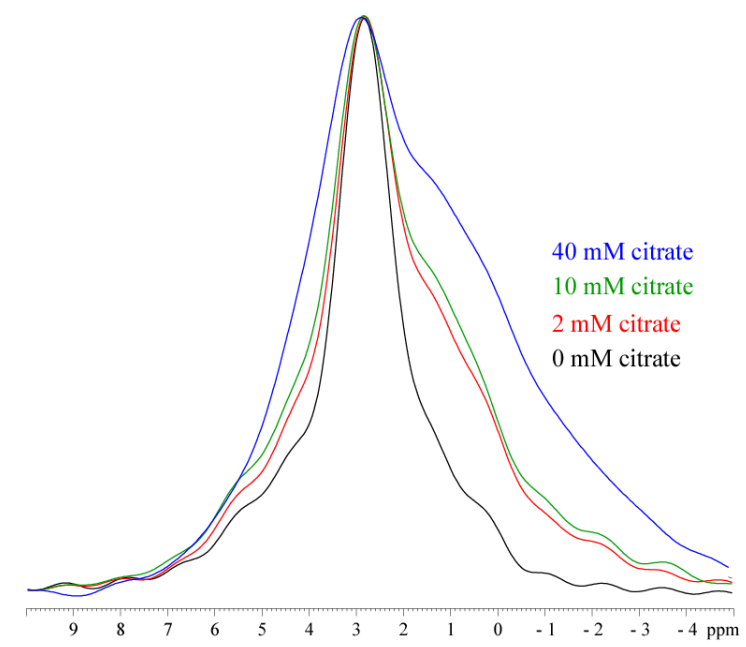

3.S1. ${ }^{31} \mathrm{P}$ cross-polarization (CP) NMR spectra of composites synthesized with Pluronic polymer, calcium phosphate, and ammonium citrate of $0,2,10$, and $40 \mathrm{mM}$ concentration.

a) $2.8 \mathrm{ppm}{ }^{31} \mathrm{P}$

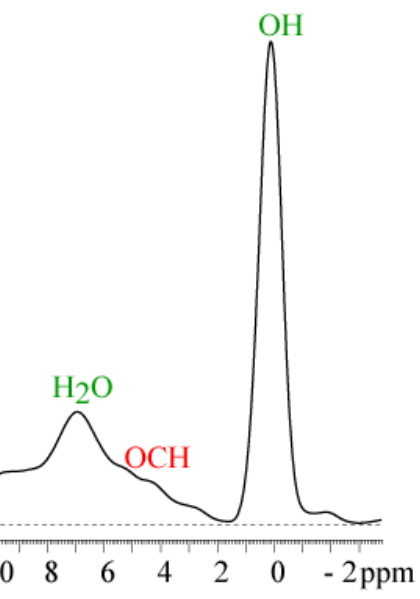

b) $1.3 \mathrm{ppm}^{31} \mathrm{P}$

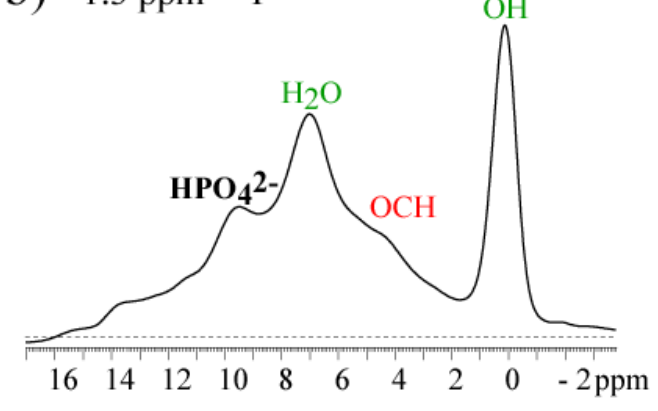

3.S2. ${ }^{1} \mathrm{H}$ spectra from cross sections taken at a) $2.8 \mathrm{ppm}$, and b) $1.0 \mathrm{ppm}{ }^{31} \mathrm{P}$ from a ${ }^{1} \mathrm{H}_{-}{ }^{31} \mathrm{P}$ HetCor spectrum of the composite synthesized with $0 \mathrm{mM}$ citrate, which contains two phosphate phases: $\mathrm{HAp}$ and $\mathrm{HPO}_{4}^{2-}$. 

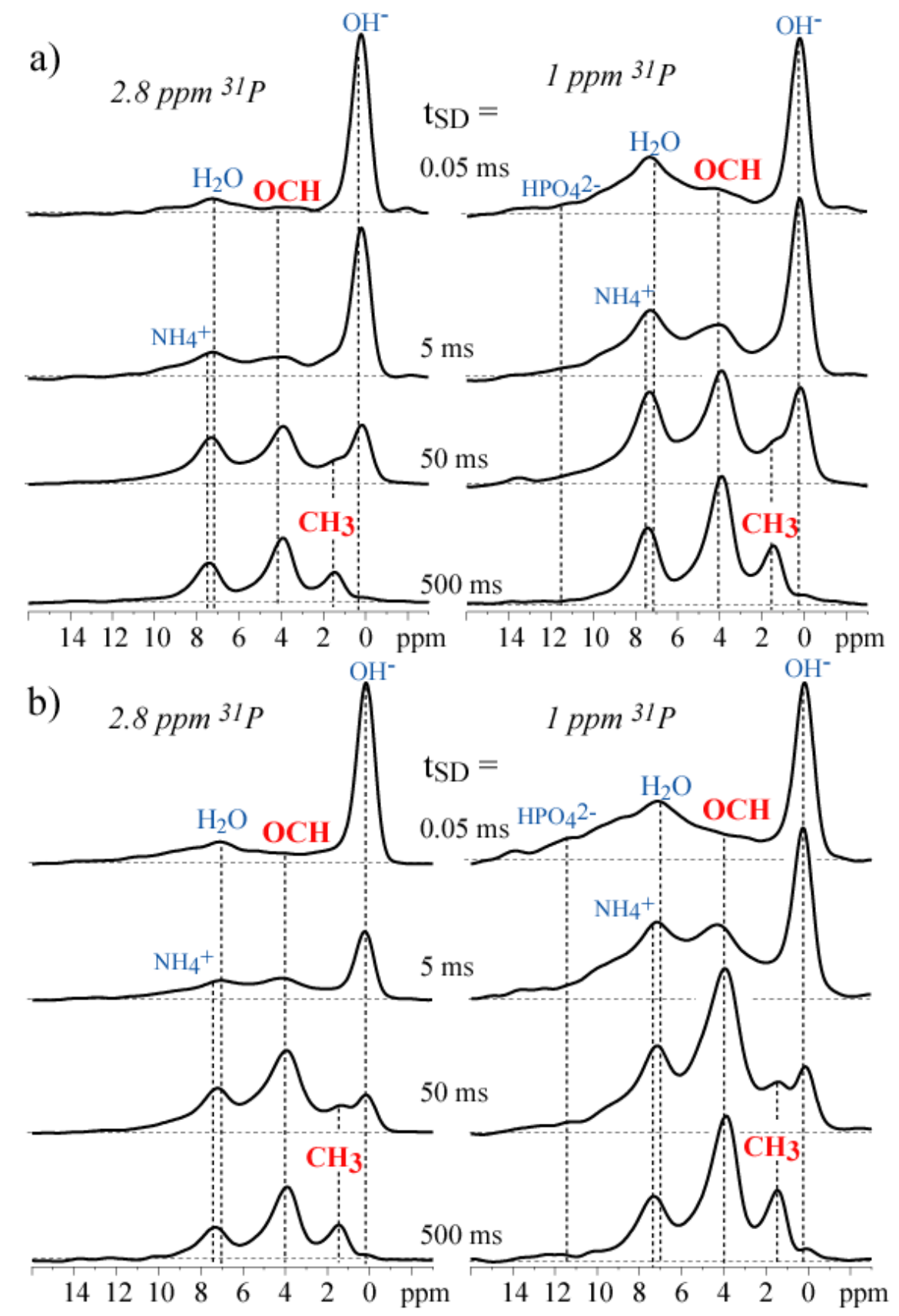

3.S3. ${ }^{1} \mathrm{H}$ spectra from cross sections taken at $2.8 \mathrm{ppm}$ and $1.0 \mathrm{ppm}{ }^{31} \mathrm{P}$ from $2 \mathrm{D}{ }^{1} \mathrm{H}_{-}{ }^{31} \mathrm{P}$

HetCor spectra of composites synthesized with Pluronic polymer, calcium phosphate, and a) $2 \mathrm{mM}$, b) $10 \mathrm{mM}$ ammonium citrate. The ${ }^{1} \mathrm{H}$ spin diffusion times are 0.05, 5, 50, and $500 \mathrm{~ms}$. 


\title{
Chapter 4
}

\section{Self-assembled calcium phosphate nanocomposites using block copolypeptide templates}

A paper published in Soft Matter

$2009,5,4311-4320$

Yanyan $\mathrm{Hu}^{\mathrm{a}}$, Yusuf Yusufoglua ${ }^{\mathrm{a}}$, Mathumai Kanapathipillai ${ }^{\mathrm{a}}$, Chu-Ya Yang ${ }^{\mathrm{b}}, \mathrm{YaQiao} \mathrm{Wu}^{\mathrm{a}}$, Papannan Thiyagarajan ${ }^{\mathrm{c}}$, Timothy Deming ${ }^{\mathrm{b}}$, Mufit Akinc ${ }^{\mathrm{a}}$, Klaus Schmidt-Rohr ${ }^{\mathrm{a}}$,

Surya Mallapragada ${ }^{\mathrm{a}}$

${ }^{a}$ Ames Laboratory, Ames, IA 50011

${ }^{b}$ Department of Bioengineering, University of California, Los Angeles, CA 90095

${ }^{c}$ Advanced Photon Source, Argonne National Laboratory, 9700 S. Cass Ave, Argonne, IL 60439

\begin{abstract}
Polylysine and polyleucine based block copolypeptides $\left(\mathrm{K}_{170} \mathrm{~L}_{30}\right)$ that form gels at very low concentrations in aqueous media are used as templates for forming self-assembled calcium phosphate nanocomposites. The synthesis method allows for simultaneous formation of the self-assembled block copolypeptide gel and of the inorganic phase, providing inorganic contents of over $50 \mathrm{wt} \%$ in the nanocomposite, approaching the inorganic content in bone. The self-assembled nanocomposites are characterized by thermogravimetric analysis, X-ray diffraction (XRD), Fourier transform infrared (FTIR) spectroscopy, solid state NMR, thermogravimetric analysis, transmission electron microscopy (TEM) and small angle X-ray scattering (SAXS). The nanocomposites formed in the presence of the block copolypeptide templates exhibit very different nanoparticle morphologies than those formed in the absence of the organic phase. Multinuclear solid state NMR methods are used to prove nanocomposite formation and characterize the secondary structure and mobility of the block copolypeptide template. The data from XRD, FTIR, ${ }^{31} \mathrm{P}$ NMR consistently show that the inorganic phase present in the nanocomposite is carbonated hydroxyapatite of nano-scale dimensions, with an elongated plate-like morphology, observed by TEM and SAXS, very similar to the mineral phase of natural bone. Overall, this approach allows a bioinspired
\end{abstract}


bottom-up approach to self-assembled hydroxyapatite nanocomposites using block copolypeptide templates, which could have applications in tissue repair.

\section{Introduction}

In biological systems, calcium phosphate ceramics are the most important inorganic components of hard tissues, and synthetic substitutes have been used in medicine and dentistry for more than 20 years. ${ }^{1}$ Among the six principal calcium salts of orthophosphoric acid, hydroxyapatite (HAp: $\left.\mathrm{Ca}_{10}\left(\mathrm{PO}_{4}\right)_{6}(\mathrm{OH})_{2}\right)$ is the best known because of its compositional and biological similarities to the mineral phase of human bone. For instance, apatite in the form of carbonated hydroxide-deficient hydroxyapatite $\left(\mathrm{CO}_{3} \mathrm{HAp}\right)$ including a variety of other minor dopants is present in natural bone to impart stiffness and hardness. ${ }^{1,2}$ It accounts for approximately $65 \mathrm{wt} \%$ of total bone mass with the remaining being mainly collagen and water. ${ }^{3,4}$ In bone formation, biomineralization produces a nanocomposite with layers of collagen molecules alternating with thin and elongated nanocrystals of carbonated apatite, ${ }^{5}$ in a process involving more than 200 different proteins. ${ }^{6}$ These proteins act as inhibitors and nucleators for the growth of apatite nanocrystals, anchored to the collagen. ${ }^{5,7}$ The combination of inorganic and organic components provides increased toughness compared to HAp alone ${ }^{8.9}$ As a result, biomaterials such as bone as well as dentine in teeth, in which the organic matrix acts as a guide and imparts toughness to the otherwise brittle inorganic phase, have attracted the interest of researchers into the investigation and development of novel organic-inorganic biocomposite materials suitable for prosthetic medical applications. ${ }^{8,10}$

Biocomposites of HAp in conjunction with various synthetic polymers ${ }^{11-16}$ and natural polymers such as collagen, ${ }^{17-20}$ chitosan, ${ }^{8,21-23}$ cellulose ${ }^{24-26}$ and gelatin ${ }^{4,27,28}$ have been investigated extensively. We have shown successful templating of calcium phosphates by self-assembling thermo-reversible cationic and Pluronic block-copolymer gels with $15 \mathrm{wt} \%$ inorganic content. ${ }^{29}$ This method enabled the formation of self-assembled nanocomposites from solution by changing the temperature. Subsequently, self-assembling thermo-reversibly gelling anionic and zwitterionic pentablock copolymers conjugated to hydroxyapatite nucleating peptides were used as templates for precipitation of calcium phosphate nanostructures. ${ }^{30}$ The inorganic fraction of the nanocomposites was increased to about $30-40$ wt $\%{ }^{31}$ The inorganic content in these nanocomposites was still significantly lower than that 
seen in nature $(65 \mathrm{wt} \%)$. The high polymer concentrations $(-25 \mathrm{wt} \%)$ needed for gel formation in these block copolymer systems is one limitation to increasing the weight fraction of the inorganic phase using these synthetic block copolymer templates. Besides our group, other researchers using similar in-situ synthesis methods also obtained polymer-HAp composites, but with relatively low inorganic content. For instance, Spanos and co-workers ${ }^{13}$ synthesized a novel composite containing $9 \mathrm{wt} \%$ of HAp and $91 \mathrm{wt} \%$ of polymer (sulfonated polysulfone), while Wang and co-workers recently fabricated nanostructured HAp in the matrix of alginate with a maximum $30 \mathrm{wt} \%$ HAp exhibiting microstructures similar to natural bone. ${ }^{32}$ The Liou group produced a poly(acrylic acid)-HAp nanocomposite of 30-32 wt $\%$ phosphate. ${ }^{33}$

In contrast, the Deming group has synthesized a family of novel block copolypeptides that form gels at very low concentrations $(\sim 0.5 \mathrm{wt} \%)$ and can serve as templates for biomineralization. ${ }^{34-37}$ This paper investigates the use of these block copolypeptides as templates for forming self-assembled calcium phosphate nanocomposites to increase the inorganic content of the nanocomposite. We demonstrate the formation of HAp/block copolypeptide $\left(\mathrm{K}_{170} \mathrm{~L}_{30}\right)$ polymer nanocomposites with a maximum inorganic content of approximately $53 \mathrm{wt} \%$. This approach is similar to nature where protein templates commonly control the nucleation and growth of the inorganic nanocrystalline phase in biomaterials such as bone.

Wet-chemical methods lend themselves to the formation of calcium phosphate ceramics with good crystallinity, physiological stability, and morphological characteristics resembling those of hard tissues. ${ }^{38}$ A number of papers have described different wet-chemical methods including chelate ${ }^{39-41}$ or urea decomposition, ${ }^{42-44}$ precipitation, ${ }^{38,45-48}$ and hydrolysis of calcium phosphate precursors. ${ }^{49-52}$ In the present study, we used precipitation onto the selfassembling block copolypeptide templates to obtain polymer-inorganic hydrogel composites. Compared to other methods, this is a relatively simple technique utilizing mild experimental conditions that preclude polymer degradation. The resulting nanocomposites have been characterized by X-ray diffraction, ${ }^{31} \mathrm{P}$ NMR, Fourier-transform infrared spectroscopy, and high-resolution transmission electron microscopy (HRTEM) to identify the phosphate phase(s) formed; by ${ }^{1} \mathrm{H}$ and ${ }^{31} \mathrm{P}$ spin-diffusion NMR to prove nanocomposite formation; by 
TEM and small-angle X-ray scattering to determine the shape of the crystallites; and by ${ }^{13} \mathrm{C}$ NMR to determine the secondary structure and mobility of the polypeptide matrix. We believe that this work may contribute towards understanding the fundamental mechanism of biomineralization that is essential for the development of novel bioinspired materials for hard tissue repair and regeneration.

\section{Results and discussion}

The polylysine segment length was determined using GPC/LS, and ${ }^{1} \mathrm{H}$ NMR was used to determine the copolymer composition. Combined, these data showed that a block copolypeptide of $\mathrm{K}_{170} \mathrm{~L}_{30}$ with low polydispersity was obtained. The consumption of the monomer and the formation of amide bonds from the polypeptide backbones $\left(1650 \mathrm{~cm}^{-1}\right.$, $1540 \mathrm{~cm}^{-1}$ ) was confirmed using FTIR measurements. ${ }^{1} \mathrm{H}$ NMR in deuterated TFA (d-TFA) indicated a 97\%-98\% removal of benzyloxycarbonyl groups from lysine residues.

The XRD patterns of the pure polymer (a), as-prepared HAp-polymer hydrogel (b), vacuum dried HAp/polymer composite (c), washed sample (after vacuum drying) (d), calcined sample (after TGA) (e), and pure HAp (Standard Reference Material ${ }^{\circledR} 2910$ ) (f) are shown in

Fig. 4.1. XRD patterns of pure polymer and pure HAp (Standard Reference Material ${ }^{\circledR} 2910$ ) are included in the XRD Fig. 4.1 to compare with that of the nanocomposite sample. For pure polymer (Fig. 4.1a), two broad humps were observed at about $2 \Theta=12^{\circ}$ and $22^{\circ}$ indicative of the amorphous nature of the polymer. The $12^{\circ}$ peak might be attributed to rod-rod packing of helical polyleucine chains. All diffraction peaks of as-prepared composite hydrogel (Fig 1b) were matched to those of pure HAp (Fig 1f) and the ICDD standard card (JCPDS 9-432). The results are also in accordance with the calcium phosphate $\mathrm{pH}$-concentration diagram ${ }^{53}$ and experimentally shown by Yusufoglu and Akinc ${ }^{41}$ that the most stable calcium phosphate phase is HAp at $\mathrm{pH}>6$. Furthermore, the broadening and overlap of the peaks (broad peak at $2 \Theta \approx 31^{\circ}$ is a summed contributions of the (211), (112) and (300) planes of HAp) is a sign of small size or/and poor crystallinity of HAp crystals in the hydrogel sample. However, the crystallographic structure of HAp observed in the as-prepared HAp-polymer hydrogel is very similar to natural bone mineral and human dentine. ${ }^{6,54,55}$ It is also worth noting that the as-prepared nanocomposite hydrogel shows a higher $\mathrm{I}_{(002)} / \mathrm{I}_{(211)}$ than that of pure HAp, indicating the preferential elongation of the crystals along the c-axis. Moreover, in addition 
to the characteristic HAp peaks, the XRD pattern of vacuum dried sample displayed some additional diffraction peaks belonging to ammonium nitrate (JCPDS 1-0809 and 76-2276) marked by solid circles in Fig. 4.1c. Further, the sharp peak at $2 \theta=29.45^{\circ}$ matches well with the most intense peak of ammonium sodium nitrate (JCPDS 28-0490). After washing the vacuum dried sample with deionized water, all diffraction peaks belonging to ammonium (sodium) nitrate disappeared (Fig. 4.1d) and left only HAp peaks. In addition, it is also worth noting that HAp is the only phase present after TGA experiments as shown in Fig. 4.1e.

\section{FTIR spectroscopic characterization of the nanocomposites}

The FTIR spectra of the pure polymer (a), vacuum dried nanocomposite (b), washed sample (after vacuum drying) (c) and pure HAp (Standard Reference Material ${ }^{\circledR} 2910$ ) (d) are shown in Fig. 4.2. The FTIR spectrum of pure HAp (Fig. 4.2d) illustrates all the characteristic bands of stoichiometric HAp. ${ }^{38,42}$ As expected, the spectrum of pure HAp exhibits phosphate peaks at $965 \mathrm{~cm}^{-1}$ (a single intense band), $470 \mathrm{~cm}^{-1}$, between $1050-1095$ $\mathrm{cm}^{-1}$ and $565,602,635 \mathrm{~cm}^{-1}$ (three sites) for $v_{1}, v_{2}, v_{3}$ and $v_{4}$ respectively.

The FTIR spectrum of pure polymer is shown in Fig. 4.2a. The possible assignments of all absorption peaks in Fig. 4.2 are provided in Table 1. Furthermore, the FTIR spectrum of vacuum dried HAp/polypeptide nanocomposite can be seen in Fig. 4.2b. The absorption bands at 566-601 $\mathrm{cm}^{-1}, 962 \mathrm{~cm}^{-1}$ and $1040-1100 \mathrm{~cm}^{-1}$ are associated with the phosphate groups of HAp. Moreover, the peaks at $1380 \mathrm{~cm}^{-1}$ and $832 \mathrm{~cm}^{-1}$ are assigned to nitrate groups, which is consistent with the results obtained from XRD. It is well established that for nitrate groups, the first absorption (at $1380 \mathrm{~cm}^{-1}$ ) is intense and broad, and the second (at 840 $\mathrm{cm}^{-1}$ ) has medium intensity and is narrow. ${ }^{56}$ As illustrated in Fig. 4.2c, the peaks associated with nitrate groups disappeared in the FTIR spectrum of the washed sample. This implies that washing of the vacuum dried HAp/polymer composite removed nitrate species, consistent with the XRD results. As seen in the FTIR spectrum of the washed sample, in addition to the characteristic HAp absorption bands, the weak features arising from the vibrational modes of the carbonate were also observed at $873,1415 \mathrm{~cm}^{-1}$ and $1462 \mathrm{~cm}^{-1}, 50,57$ suggesting that the HAp contains carbonate ions. While the $\mathrm{CO}_{3}{ }^{2-}$ ions can replace $\mathrm{OH}^{-}(\mathrm{A}-$ type substitution) or $\mathrm{PO}_{4}{ }^{3-}$ (B-type substitution) in the $\mathrm{HAp}$ structure, the $\mathrm{CO}_{3}{ }^{2-}$ peaks shown 
in Fig. 4.2c are assigned only to the B-type substitution $\left(\mathrm{CO}_{3}{ }^{2-}\right.$ ions replaces $\left.\mathrm{PO}_{4}{ }^{3-}\right) .{ }^{50}$ The $\mathrm{CO}_{3}{ }^{2-}$ substitution at the $\mathrm{PO}_{4}{ }^{3-}$ site in HAp crystals is commonly observed for preparation in aqueous systems, ${ }^{58}$ as has been also observed in the present work. As the apatite in human bone also contains $\mathrm{CO}_{3}{ }^{2-}$ ions at about 4-8 wt\% depending upon age, its presence in the HAp structure is beneficial. ${ }^{6,59}$ It has been reported that at low concentration, $\mathrm{CO}_{3}{ }^{2-}$ ions substantially improve the mechanical strength of apatite ${ }^{59}$ and the presence of $\mathrm{CO}_{3}{ }^{2-}$ ions in the HAp structure also influences the decomposition, sinterability, solubility and biological reactivity of the apatite. ${ }^{47}$ Since no carbonate source was present in the starting materials, carbonate incorporation into the HAp might be due to the atmospheric carbon dioxide as has been observed during mineral precipitation in carbonate-free solutions. ${ }^{25,60}$ Further, broad absorption bands centered around 3450 and $1640 \mathrm{~cm}^{-1}$ are due to incorporated water molecules ${ }^{53,61}$ which might also be present in Fig. $4.2 \mathrm{a}$ and $2 \mathrm{~b}$ along with the polymer related peaks in the same spectral region.

The TGA results of as-prepared HAp/polypeptide hydrogel, vacuum dried HAp/polypeptide nanocomposite, washed sample and pure HAp (Standard Reference Material@ 2910) are illustrated in Fig. 4.3. As can be seen from the trace of as-prepared HAp/polypeptide hydrogel, there is a sharp weight decrease between 70 and $175^{\circ} \mathrm{C}$, which is assigned to the loss of free and bound water. After vacuum drying, most of the water is removed (Fig. 4.3b). The weight loss in the $200-450{ }^{\circ} \mathrm{C}$ range is due to thermal decomposition of polymer and perhaps ammonium nitrate (as shown in Fig. 4.3c, weight loss between 200 and $450{ }^{\circ} \mathrm{C}$ is decreased for washed sample after removing ammonium nitrate). There is no change in sample weight above $500{ }^{\circ} \mathrm{C}$, indicating that the polymer and nitrate species have decomposed completely by $500{ }^{\circ} \mathrm{C}$. It is well established that HAp is thermally stable below $1000{ }^{\circ} \mathrm{C}^{23}$ and there is almost no weight change for pure HAp between $50-570$ ${ }^{\circ} \mathrm{C}$, as shown in Fig. 4.3a. As a result, the HAp content for the $\mathrm{CaP} /$ polymer nanocomposite is determined to be approximately $53 \mathrm{wt} \%$ based on the dried sample at $175^{\circ} \mathrm{C}$. This is consistent with the theoretical weight ratio of HAp/block copolypeptide in the composite calculated from the amount of hydroxyapatite formed based on eqn (4.1). 


\section{NMR identification and quantification of phosphates}

Fig. 4.4 shows direct-polarization (DP) and cross-polarization (CP) ${ }^{31} \mathrm{P}$ NMR spectra of the synthesized composite. The DP spectrum shows one dominant band, whose chemical shift (2.8 ppm) agrees with that of hydroxyapaptite, ${ }^{62}$ though the linewidth is larger than for ideal HAp. The CP spectrum, which gives higher intensity to the phosphates close to protons, presents a different lineshape from DP, indicating the existence of more than one phosphate species. The broader base and significant intensity increase of the right shoulder of the CP spectrum suggests the presence of $\mathrm{HPO}_{4}{ }^{2-}$ or of $\mathrm{PO}_{4}{ }^{3-}$ hydrogen bonded to $\mathrm{H}_{2} \mathrm{O}$, in addition to apatitic $\mathrm{PO}_{4}{ }^{3-}$.

These assignments can be confirmed by recording ${ }^{31} \mathrm{P}$ DP spectra with $0 \mathrm{~ms}$ and 0.28 ms of recoupled ${ }^{1} \mathrm{H}^{-31} \mathrm{P}$ dipolar dephasing, see Fig. 4.S1a (in the ESI), where a more distinct lineshape results from the different dephasing behavior of $\mathrm{PO}_{4}^{3-}$ and $\mathrm{HPO}_{4}{ }^{2-}$. Provided similar mobility, the signal of phosphates that are protonated or hydrogen bonded will dephase faster than that of nonprotonated ones. The dephasing curves obtained from the gated decoupling experiments are shown in Fig. 4.S1b. The difference in dephasing rate shows that there are two different phosphate components. One is not protonated, with very slow dephasing, and the other is monoprotonated or hydrogen-bonded to a proton.

Based on the differential dephasing of the phosphate signals, the DP/MAS spectrum can be deconvoluted into two different components as shown in Fig. 4.4a (dashed lines). The sharp component is $\mathrm{PO}_{4}{ }^{3-}$ of hydroxyapatite, accounting for $68 \%$ of all phosphates, the broad one is $\mathrm{HPO}_{4}{ }^{2-}$ or hydrogen-bonded $\mathrm{PO}_{4}{ }^{3-}$, accounting for $32 \%$.

\section{NMR validation of nanocomposite formation}

The one-dimensional spectra show that there are two types of phosphates present in the sample, but do not reveal whether they are part of nanocomposites. This information can be obtained by two-dimensional ${ }^{1} \mathrm{H}-{ }^{31} \mathrm{P}$ heteronuclear correlation NMR experiments with ${ }^{1} \mathrm{H}$ spin diffusion. Cross peaks between ${ }^{1} \mathrm{H}$ from the organic phase and ${ }^{31} \mathrm{P}$ from the inorganic phase are characteristic of nanocomposites. ${ }^{29,63}$ For nanocomposites, the polymer proton peaks will show up within tens to hundreds of milliseconds. In Fig. 4.5, the spin diffusion from polymer protons to protons in the inorganic phase is indeed observed within $50 \mathrm{~ms}$ and 
equilibrium is reached within $500 \mathrm{~ms}$. In samples with larger domains or for short spin diffusion times, the phosphates only "see" the protons close by, i.e. in the inorganic phase.

The series of ${ }^{1} \mathrm{H}-{ }^{31} \mathrm{P}$ correlation spectra in Fig. 4.5a were acquired with homonuclear decoupling in the ${ }^{1} \mathrm{H}$ dimension. Fig. $4.5 \mathrm{~b}$ displays ${ }^{1} \mathrm{H}$ cross sections from the 2D spectra at $2.8 \mathrm{ppm}^{31} \mathrm{P}$, which demonstrate the spin diffusion process. On the time scale of $0.05 \mathrm{~ms}$, phosphates only "see" $\mathrm{OH}^{-}, \mathrm{HPO}_{4}{ }^{2-}$ and $\mathrm{H}_{2} \mathrm{O}$ protons. After $50 \mathrm{~ms}$, the peak of a different $\mathrm{H}_{2} \mathrm{O}$ species shows up, and within $500 \mathrm{~ms}$, the $\mathrm{CH}$ and $\mathrm{CH}_{2}$ proton signals come up along with a significant $\mathrm{H}_{2} \mathrm{O}$ peak. The $\mathrm{NH}_{4}{ }^{+}$peak of the polymer should also appear, but is overlapped by the dominant $\mathrm{H}_{2} \mathrm{O}$ peak. ${ }^{64}$ The completion of ${ }^{1} \mathrm{H}$ spin diffusion within $500 \mathrm{~ms}$ is signified by the disappearance of $\mathrm{OH}^{-}$and $\mathrm{HPO}_{4}{ }^{2-}$ proton peaks. This proves the formation of a nanocomposite. Fig. $4.5 \mathrm{c}$ reveals another ${ }^{1} \mathrm{H}$ environment associated with $\mathrm{HPO}_{4}{ }^{2-}$ or $\mathrm{PO}_{4} \cdot \mathrm{H}_{2} \mathrm{O}$. The appearance of the polymer proton peaks within $50 \mathrm{~ms}$ indicates fast spin diffusion, which is the evidence of nanocomposite formation. Therefore, both types of phosphates, hydroxyapatite $\mathrm{PO}_{4}{ }^{3-}$ and $\mathrm{HPO}_{4}{ }^{2-}$, are part of a nanocomposite.

Fig. 4.6a shows a one-pulse ${ }^{1} \mathrm{H}$ spectrum with background suppression. ${ }^{65}$ Several resolved peaks can be assigned according to their chemical shifts. $\mathrm{H}_{2} \mathrm{O}$ and $\mathrm{NH}_{3}{ }^{+}$peaks are centered at 7 ppm with a broad envelope; 7 ppm is the typical chemical shift of bound $\mathrm{H}_{2} \mathrm{O}$, while bulk water resonates at $5 \mathrm{ppm}$. After 18 hours of intensive vacuum drying of the samples at $105^{\circ} \mathrm{C}$ in order to remove the mobile $\mathrm{H}_{2} \mathrm{O}$ component, the $7 \mathrm{ppm}$ peak shifted by $0.2 \mathrm{ppm}$ and lost about $1 / 3$ of its intensity, which means that the majority of $\mathrm{H}_{2} \mathrm{O}$ in this composite is bound water. The broad peak of strongly bound water was observed at $0.05 \mathrm{~ms}$ in Fig. 4.6b, indicating that the strongly bound water has intimate contact with the inorganic phase. The sharp peak of the weakly bound $\mathrm{H}_{2} \mathrm{O}$ became dominant after $50 \mathrm{~ms}$ spin diffusion, which means that the weakly bound water is also close to the inorganic phase. After extensive drying, the previously resolved $\mathrm{CH}$ and $\mathrm{CH}_{2}$ polypeptide peaks became invisible in the one-pulse ${ }^{1} \mathrm{H}$ spectrum, see Fig. 4.6a (dashed line), which suggests that the removal of mobile $\mathrm{H}_{2} \mathrm{O}$ reduces the mobility of the peptide. A band of $\mathrm{NH}_{4}{ }^{+}$, which happens to also resonate around $7 \mathrm{ppm}$, is present in the ${ }^{1} \mathrm{H}$ spectrum as a shoulder to the right of the intense water peak. ${ }^{1} \mathrm{H} \mathrm{T}_{1}$ measurements have shown that $\mathrm{NH}_{4}{ }^{+}$has a longer $\mathrm{T}_{1}$ than the components of the nanocomposite, proving that it forms a separate phase $\left(\mathrm{NH}_{4} \mathrm{NO}_{3}\right)$. 
The ${ }^{1} \mathrm{H}-{ }^{31} \mathrm{P}$ heteronuclear correlation experiments were also performed without ${ }^{1} \mathrm{H}$ homonuclear decoupling. Fig. 4.6 (b,c) displays cross sections from the 2D spectra (not shown) taken at $2.5 \mathrm{ppm}$ and $0.5 \mathrm{ppm}$ in the ${ }^{31} \mathrm{P}$ dimension. Without spin diffusion, the $\mathrm{OH}^{-}$ proton peak at $0.2 \mathrm{ppm}$ in Fig. $4.6 \mathrm{~b}$ confirms the peak assignment of hydroxyapatite in the ${ }^{31} \mathrm{P}$ spectra of Fig. 4. 4. The strongly bound $\mathrm{H}_{2} \mathrm{O}$ peak is not well resolved due to ${ }^{1} \mathrm{H}-{ }^{1} \mathrm{H}$ homonuclear coupling, while the weakly bound $\mathrm{H}_{2} \mathrm{O}$ presents a sharp peak on the broad base of the strongly bound $\mathrm{H}_{2} \mathrm{O}$. The $\mathrm{HPO}_{4}{ }^{2-}$ peak seen in Fig. $4.6 \mathrm{c}$ is not visible, likely also due to strong ${ }^{1} \mathrm{H}-{ }^{1} \mathrm{H}$ homonuclear coupling, which is common for interfacial $\mathrm{HPO}_{4}{ }^{2-}$ species.

The $2 \mathrm{D}$ heteronuclear correlation experiments prove that both $\mathrm{HPO}_{4}{ }^{2-}$ and hydroxyapatite are part of the nanocomposites, but do not establish whether they are in the same phase. This can be achieved instead by a one-dimensional ${ }^{31} \mathrm{P}$ spin diffusion experiment. If these two kinds of phosphates are in different phases, no ${ }^{31} \mathrm{P}$ spin diffusion between them will occur on the $10 \mathrm{~s}$ time scale. Conversely, fast spin diffusion from $\mathrm{HPO}_{4}{ }^{2-}$ to hydroxyapatite proves that they are part of the same particle. After selection of $\mathrm{HPO}_{4}{ }^{2-}{ }^{31} \mathrm{P}$ magnetization by short cross polarization, narrowing of the line due to ${ }^{31} \mathrm{P}$ spin diffusion to $\mathrm{PO}_{4}{ }^{3-}$ is actually observed within $1 \mathrm{~s}$ (Fig. 4.7), confirming that $\mathrm{HPO}_{4}{ }^{2-}$ and hydroxyapatite are in the same phase and separated by less than $10 \mathrm{~nm}$. Most likely, $\mathrm{HPO}_{4}{ }^{2-}$ is at the interface of the inorganic phase with the polymer.

\section{NMR characterization of the polypeptide phase in the nanocomposite}

${ }^{13} \mathrm{C}$ NMR experiments have been carried out to study the conformation and mobility of polypeptide in the synthesized nanocomposite. The ${ }^{13} \mathrm{C}$ spectrum is shown in Fig. 4.8a (thin line). Peak assignments and literature values of solid-state ${ }^{13} \mathrm{C}$ NMR chemical shifts for $\alpha$-helix and $\beta$-sheet conformations are marked above the spectra for ref. 66,67 . The chemical shifts indicate that the hydrophobic leucine domain is $\alpha$-helical and that most lysine segments adopt a $\beta$-sheet conformation, while the rest is in a random-coil. This is consistent with the results of Deming and co-workers who have demonstrated that in hydrogels, $90 \%$ of the leucine domains of polypeptide $\mathrm{K}_{160} \mathrm{~L}_{40}$ and $\mathrm{K}_{180} \mathrm{~L}_{20}$ formed $\alpha$-helices. ${ }^{70}$ This shows that the $\alpha$-helical structure of the polyleucine domain ${ }^{68}$ is retained even after the synthesis of the nanocomposite and vacuum drying. The peak intensities and the positions of the $\mathrm{C}_{\alpha}$ and $\mathrm{C}_{\beta}$ 
signals are not consistent with the alternative assumption that the lysine segments are $\alpha$ helical.

A corresponding $\mathrm{CP}{ }^{13} \mathrm{C}$ NMR spectrum after $40 \mu$ s of gated decoupling was recorded in order to selectively detect mobile components of the polypeptide. High mobility will significantly reduce the $\mathrm{C}-\mathrm{H}$ dipolar coupling, resulting in residual ${ }^{13} \mathrm{C}$ signals after gated decoupling. The resulting spectrum (thick line) in Fig. 4.8a indicates that the backbone of the polypeptide is rigid, since all the $\mathrm{C}_{\alpha}$ signals for both leucine and lysine are completely dephased. The side chain carbons $\mathrm{C}_{\gamma}, \mathrm{C}_{\delta}$ and $\mathrm{C}_{\varepsilon}$ show significant residual intensity, which means that the side chains of the polypeptide are quite mobile.

Motions on the $\mathrm{MHz}$ scale generally induce fast $\mathrm{T}_{1}$ relaxation, which enables another method to detect the mobile components. After direct polarization (DP) with a short recycle delay of $1 \mathrm{~s}$, only signals of carbons with short $\mathrm{T}_{1} \mathrm{~s}$ are detected, see Fig. 4.8(b). Signals of all mobile carbons that are seen after gated decoupling (Fig. 4.8a, thick line) are also present in Fig. 4.8(b), confirming the conclusions about mobility. Besides, the absence of the carbonyl $(\mathrm{C}=\mathrm{O})$ and $\mathrm{C}_{\alpha}$ signals in Fig. $4.8 \mathrm{~b}$ confirms the rigidity of the polypeptide backbone.

However, the 70:30 signal intensity ratio of the rigid backbone carbons of lysine components to those of leucine in the CP spectrum of Fig. 4.8a does not match with the 85:15 stoichiometry of $\mathrm{K}_{170} \mathrm{~L}_{30}$, which suggests some mobile lysine residues in the regularly vacuum dried sample are not detected in our CP spectra. In order to make these "invisible" components detectable, the intensively dried sample was studied. The ${ }^{13} \mathrm{C}$ spectra after $\mathrm{CP}$ and $\mathrm{CP}$ with gated decoupling are shown in Fig. 4.9. Changes in the $\mathrm{CP}$ spectrum upon intensive drying are indeed observed. First, $\mathrm{C}=\mathrm{O}$ signals of random-coil lysine that do not show up in Fig. 4.8a, due to high mobility and resulting low CP efficiency, appear at 176 ppm after drying in Fig. 4.9. Second, the $\mathrm{C}_{\alpha}$ peak of random-coil lysine that appears as a shoulder next to the $\mathrm{C}_{\alpha}$ signal of $\alpha$-helix leucine in Fig. 4.8a now shows up intensely in Fig. 4.9 (thin line) and overlaps with the $\mathrm{C}_{\alpha}$ peak from leucine. Above all, the ratio of lysine to leucine backbone carbons obtained from the $\mathrm{CP}$ spectrum is approaching the theoretical ratio due to the contribution of the previously "invisible" random-coil lysine. Moreover, the CP spectrum (thin line) in Fig. 4.9 is less resolved than in Fig. 4.8a because of more peaks showing up and less dynamic conformational averaging. Furthermore, the reduced mobility 
is confirmed by the gated decoupling spectrum shown in Fig. 4.9 (thick line). The $\mathrm{C}_{\alpha}$ peak of random-coil lysine vanishes, indicating that the backbone of random-coil lysine has become rigid after intensive drying. The decreased intensity of the residual signals in CP with gated decoupling in Fig. 4.8 compared to Fig. 4.9 confirms that the side chains are less mobile as well.

\section{TEM of the nanocomposites}

The TEM micrographs of the HAp crystals formed in the block copolypeptide gel and those obtained by direct synthesis from an aqueous solution are shown in Fig. 4.10. The HAp particles in the nanocomposite exhibit thin elongated plate-like morphology with a mean length of about 50-110 nm, width of 10-20 nm, and are about $3 \mathrm{~nm}$ thick (Fig. 4.10a). The elongated plate-like morphology of the HAp nanocrystals can also be seen from the STEM high angle annular dark field (HAADF) image in Fig. 4.10b. Appearance of needle-like morphology (Fig. 4. 10a and b) is due to the on-edge viewing angle of the curled plate-like crystallites. Further, the morphology of HAp observed in the present study is quite similar to the apatite crystals found in human woven bone and mineralized dentin. ${ }^{69,70}$ In general, bone mineral consists of plate-like crystals with average dimensions $\sim 50-100 \mathrm{~nm}$ or more in length, $\sim 25-50 \mathrm{~nm}$ in width, $\sim 2-6 \mathrm{~nm}$ in thickness ${ }^{60,69,71}$ and their small size is a crucial factor related to the solubility. ${ }^{6}$ The SAED pattern of the as-prepared hydrogel sample given as an inset in Fig. 4.10a demonstrates well-defined broad ring patterns proving polycrystalline and nano-size nature of HAp phase. The observed diffraction rings can be indexed to HAp and these observations are in good agreement with the XRD and FTIR results. Moreover, Fig. 4.10c shows the bright-field TEM image of the washed sample (after vacuum drying) with the corresponding SAED pattern and it can be seen from the micrograph that the morphology of the HAp nanoparticles is very similar to that observed in the as-prepared hydrogel nanocomposite. In addition, it is also worth noting that the apatite crystals in the nanocomposites appear elongated plate-like, while the material prepared in the absence of polymer exhibit crystals with irregular shapes (Fig. 4.10d). Furthermore, the SAED pattern of HAp particles prepared in the absence of polymer shows many spots that do not form well 
defined rings (except from the (112) plane), indicating larger HAp crystals compared to the as-prepared nanocomposite sample.

Bright-field (BF) and dark-field (DF) transmission electron micrographs of the HAp/polypeptide nanocomposite taken from same area are displayed in Fig. 4.11a and 11b, respectively. The lower inset in Fig. 4.11a shows the corresponding SAED pattern with (300) and (002) diffraction rings of HAp. The DF-TEM image in Fig. 4.11b was obtained by isolating the HAp (002) diffraction in the nanocomposite. The mineral phase (HAp) appears as highlights in Fig. 4.11b, showing the HAp nanocrystals distribution in the amorphous polymer matrix. Moreover, the atomic calcium to phosphorus ratio of the inorganic phase was found to be $1.71 \pm 0.07$ by STEM-EDS measurements, which is in accordance with the stoichiometric value of pure $\mathrm{HAp}\left(\mathrm{Ca}_{10}\left(\mathrm{PO}_{4}\right)_{6}(\mathrm{OH})_{2}\right)$. The slight increase in $\mathrm{Ca} / \mathrm{P}$ ratio of HAp over 1.67, the stoichiometric ratio, could be due to the carbonate substitution of the phosphate, consistent with FTIR results.

High-resolution TEM (HRTEM) analysis of the nanocomposites also confirmed the existence of HAp in the nanocomposite. The lattice fringe measurements from the HRTEM micrographs in Fig. $4.11 \mathrm{c}$ and $11 \mathrm{~d}$ revealed $d$ spacings of 3.44, 2.81, and $2.72 \AA$, corresponding to the (002), (211) and (300) HAp reflections, respectively. The upper inset in Fig. 4.11c demonstrates the filtered inverse-FFT image with (002) HAp planes from the indicated area of the HRTEM image. In addition, Fig. 4.11d shows the (002) and (211) HAp planes with an interplanar angle of $\sim 66^{\circ}$, and the (002) and (300) HAp planes with an interplanar angle of $90^{\circ}$, which are consistent with the calculated values of HAp (JCPDS 9432, a hexagonal unit cell (space group $\mathrm{P}_{3} / \mathrm{m}$ ) with unit cell dimensions $\mathrm{a}=\mathrm{b}=9.418 \AA$ and $c=6.884 \AA$ ). The HRTEM image of elongated plate-like nanocrystals in longitudinal sections (Fig. 4.11c) revealed a lattice spacing of $3.44 \AA$ (corresponding to the interplanar spacing of (002) planes for hexagonal HAp), indicating that the growth of the plate-like nanocrystals occurs in the (001) direction. The intense (002) reflection in XRD (Fig. 4.1) also indicates that the c-axis is the preferred growth direction, which is similar to bone where nano-size apatite plate-like crystals associated with the collagen fibrils show strong preferred orientation in their c-axis. ${ }^{1,54,69}$ 


\section{SAXS characterization}

The nanocomposites were characterized with small angle X-ray scattering. The nanocomposites exhibited power law scattering (Fig. 4.12) similar to that observed in bone. ${ }^{72,73}$ The characteristic length scale of the particles was found to be around $3 \mathrm{~nm}$. In the SAXS data we observe signatures for the average size of the inorganic nanocrystals in the high $\mathrm{Q}$ region and power law scattering in the low $\mathrm{Q}$ region consistent ${ }^{72,73}$ with the elongated particle morphologies observed in TEM. Further, modified Guinier analysis for the cross sectional dimensions shows the thickness of the particles to be around $3 \mathrm{~nm}$, consistent with the TEM measurements.

\section{Materials and methods}

All materials used were obtained from either Sigma Aldrich or Fisher Scientific and were of laboratory grade and purity.

\section{Polypeptide synthesis}

The polypeptide $\mathrm{K}_{170} \mathrm{~L}_{30}$ was synthesized by the previously developed procedures. ${ }^{68}$ Briefly, first $\mathrm{N}_{\varepsilon}$-CBZ-L-lysine and L-leucine monomers were converted to $\alpha$-aminoacid-Ncarboxyanhydrides (NCAs) to facilitate ring-opening polymerization. The NCA preparation was carried out with phosgene at $40{ }^{\circ} \mathrm{C}$ in dry tetrahydrofuran. The monomers were then reacted with $\left(\mathrm{PMe}_{3}\right)_{4} \mathrm{Co}$ initiator in THF and the polypeptide copolymerization was carried out under inert conditions at room temperature for 1 hour with sequential addition of the NCA monomers. The polymers were then dissolved in trifluoroacetic acid and deprotected by adding $33 \% \mathrm{HBr}$ in acetic acid to solutions of copolypeptide in trifluoroacetic acid at $0{ }^{\circ} \mathrm{C}$ for 1 hour. The deprotected polymers were then dissolved in deionized water, dialyzed, and freeze dried. Polymer molecular weight and other chemical characteristics were determined by nuclear magnetic resonance (NMR), Fourier-transform infrared (FTIR) spectroscopy and gel permeation chromatography (GPC). FTIR was used to verify the consumption of the monomer and the formation of amide bonds in the polypeptide backbones. Tandem gel permeation chromatography/light scattering (GPC/LS) was performed at $60^{\circ} \mathrm{C}$ using an SSI Accuflow Series III pump equipped with Wyatt DAWN EOS light scattering and Optilab 
rEX refractive index detectors. Separations were achieved using $10^{5}, 10^{4}$, and $10^{3} \AA$ Phenomenex Phenogel $5 \mu \mathrm{m}$ columns with 0.1 M LiBr in DMF as eluent and sample concentrations of $5 \mathrm{mg} / \mathrm{mL}$. The deprotection of the polymers was then verified using ${ }^{1} \mathrm{H}$ NMR.

\section{Block copolypeptide-hydroxyapatite nanocomposite synthesis}

To prepare calcium phosphate (CaP)-polypeptide nanocomposite hydrogel samples, the following procedure was used: $0.11 \mathrm{~g} \mathrm{~K}_{170} \mathrm{~L}_{30}$ was mixed with $1.5 \mathrm{~mL} 4.0 \mathrm{M}$ $\mathrm{Ca}\left(\mathrm{NO}_{3}\right)_{2} \cdot 4 \mathrm{H}_{2} \mathrm{O}$ solution. To dissolve the polypeptide and form the hydrogel, the calcium containing polymer solution was kept at room temperature for two days. Then, $0.9 \mathrm{~mL} 4.0 \mathrm{M}$ $\left(\mathrm{NH}_{4}\right)_{2} \mathrm{HPO}_{4}$ solution was added and aged at room temperature for an additional four days to form a CaP-hydrogel nanocomposite containing approximately $4.0 \mathrm{wt} \%$ polymer. In addition to the polypeptide and the inorganic phase, the gel contains mainly water along with some ammonium nitrate. The $\mathrm{pH}$ of the hydrogel sample was adjusted to $\sim 8$ from an initial $\mathrm{pH}$ of around 3 by adding $\mathrm{NaOH}$ solution. The formation of $\mathrm{HAp}$ in $\mathrm{CaP} /$ polymer hydrogel is believed to take place through the following simplified chemical reaction: $10 \mathrm{Ca}\left(\mathrm{NO}_{3}\right)_{2}+6\left(\mathrm{NH}_{4}\right)_{2} \mathrm{HPO}_{4}+2 \mathrm{H}_{2} \mathrm{O} \rightarrow \mathrm{Ca}_{10}\left(\mathrm{PO}_{4}\right)_{6}(\mathrm{OH})_{2}+12 \mathrm{NH}_{4} \mathrm{NO}_{3}+8 \mathrm{HNO}_{3}$

\section{Characterization}

Calcium phosphate nanocomposite samples were analyzed by using techniques of $\mathrm{X}$ ray diffraction (XRD), FTIR spectroscopy, thermogravimetric analysis (TGA), NMR, small angle X-ray scattering (SAXS), transmission electron microscopy (TEM), high resolution transmission electron microscopy (HRTEM), scanning transmission electron microscopy (STEM). Moreover, the atomic $\mathrm{Ca} / \mathrm{P}$ ratio was also monitored by energy dispersive $\mathrm{X}$-ray spectroscopy (EDS).

The structure and crystallinity of the as-prepared nanocomposite samples were investigated by a powder X-ray diffractometer (X'Pert PRO, PANalytical Inc., Westborough, MA, USA) using monochromatic $\mathrm{CuK} \alpha$ radiation $(\lambda=0.15418 \mathrm{~nm})$ and operating at $45 \mathrm{kV}$ and $40 \mathrm{~mA}$. The scanning rate was $0.008^{\circ} / \mathrm{s}$ with a step size of $0.017^{\circ}$ over a range of $10^{\circ} \leq$ 
$2 \theta \leq 60^{\circ}$. Scintag DMSNT search/match software and the ICDD 2007 database were used for the phase analysis.

Chemical analysis of the samples was carried out by a Bruker FTIR spectrometer (Bruker IFS-66v, Bruker Optics Inc., Billerica, MA, USA) over the range between 400-4000 $\mathrm{cm}^{-1}$ with a resolution of $4 \mathrm{~cm}^{-1}$ and averaging 32 scans. NMR studies (see below) require vaccum dried samples; therefore the XRD patterns of vaccum dried samples both before and after washing were obtained, to confirm the removal of ammonium nitrate during washing.

For the FTIR analysis of all the different samples mentioned above, the samples were pressed into $\mathrm{KBr}$ pellets containing approximately $1 \mathrm{wt} \%$ sample. The inorganic phase (HAp) content of the nanocomposites was estimated with a Perkin Elmer thermogravimetric analyzer (Perkin Elmer, TGA 7, Downers Grove IL, USA). Approximately $50 \mathrm{mg}$ of the gel sample, the vaccum dried nanocomposite and the washed sample after vaccum drying, were heated to $50{ }^{\circ} \mathrm{C}$ under air flow conditions, held at this temperature for $1 \mathrm{~min}$, then ramped to $120^{\circ} \mathrm{C}$ at a rate of $5.0^{\circ} \mathrm{C} / \mathrm{min}$, and at a rate of $10.0^{\circ} \mathrm{C} / \mathrm{min}$ to $550^{\circ} \mathrm{C}$. XRD patterns and FTIR spectra of the calcined samples, after being subject to thermogravimetric analysis, were also obtained.

Solid state NMR. Solid state NMR experiments were performed using a Bruker DSX400 spectrometer (Bruker-Biospin, Rheinstetten, Germany) at $400 \mathrm{MHz}$ for ${ }^{1} \mathrm{H}, 162 \mathrm{MHz}$ for ${ }^{31} \mathrm{P}$ and $100 \mathrm{MHz}$ for ${ }^{13} \mathrm{C}$ with a Bruker $4 \mathrm{~mm}$ magic-angle spinning double resonance probehead at ambient temperature. All samples were analyzed without washing, but were vacuum dried before NMR experiments because solid state NMR requires relatively dry samples to avoid power absorption by ionic conduction. The $90^{\circ}$ pulse lengths were $4 \mu$ s for ${ }^{1} \mathrm{H}, 4.7 \mu \mathrm{s}$ for ${ }^{31} \mathrm{P}$ and $4 \mu \mathrm{s}$ for ${ }^{13} \mathrm{C}$. All the ${ }^{31} \mathrm{P}$ and ${ }^{1} \mathrm{H}$ NMR experiments were carried out at a spinning speed of $6.5 \mathrm{kHz}$, while ${ }^{13} \mathrm{C}$ NMR experiments were at $4 \mathrm{kHz} .{ }^{31} \mathrm{P}$ direct polarization experiments, including those with gated recoupling, were performed with recycle delays of $100 \mathrm{~s} .{ }^{1} \mathrm{H}-{ }^{31} \mathrm{P}$ correlation experiments without ${ }^{1} \mathrm{H}$ homonuclear decoupling, using the wideline separation (WISE) NMR pulse sequence with hypercomplex data acquisition, were carried out with 256 $20 \mu \mathrm{s}_{1}$ increments and $2 \mathrm{~s}$ recycle delays. $2 \mathrm{D}{ }^{1} \mathrm{H}-{ }^{31} \mathrm{P}$ heteronuclear correlation (HetCor) NMR experiments were carried out with $10044 \mu \mathrm{s}_{1}$ increments of FSLG homonuclear 
decoupling and $2 \mathrm{~s}$ recycle delays. Lee-Goldburg cross polarization (LGCP) was used for polarization transfer from ${ }^{1} \mathrm{H}$ to ${ }^{31} \mathrm{P}$ in both "WISE" ${ }^{74}$ and HetCor experiments with contact times of $0.7 \mathrm{~ms}$ and $0.5 \mathrm{~ms}$, respectively. $1 \mathrm{D}^{31} \mathrm{P}$ spin diffusion experiments after $0.1 \mathrm{~ms}$ of cross polarization were accomplished with spin diffusion times of $0.1,0.5,1,5,10,50,100$, $500 \mathrm{~ms}$, and $1 \mathrm{~s} .{ }^{13} \mathrm{C}$ cross polarization and cross polarization with gated decoupling of $40 \mu \mathrm{s}$ were used to detect the mobile components in the polypeptide phase. These ${ }^{13} \mathrm{C}$ NMR experiments were carried out with recycle delays of $2 \mathrm{~s}$ and 45056 scans. ${ }^{1} \mathrm{H}$ one-pulse NMR spectrum was obtained with $3.8 \mu \mathrm{s}{ }^{1} \mathrm{H} 90^{\circ}$ pulse and background suppression. Two-pulse phase-modulation (TPPM) was used for heteronuclear dipolar decoupling in all these experiments. The proton peak at $0.18 \mathrm{ppm}$ and ${ }^{31} \mathrm{P}$ peak at $2.8 \mathrm{ppm}$ of NIST hydroxyapatite were used as secondary references to calibrate the ${ }^{1} \mathrm{H}$ and ${ }^{31} \mathrm{P}$ chemical shifts, respectively. The $\mathrm{C}=\mathrm{O}$ carbon peak at $176.49 \mathrm{ppm}$ of $25 \%{ }^{13} \mathrm{C}$ labeled $\alpha$-glycine was used for ${ }^{13} \mathrm{C}$ chemical shift calibration.

Electron microscopy. STEM, HRTEM, Selected-Area Electron Diffraction (SAED), and EDS studies were performed with a FEI-Tecnai G² F20 (FEI Inc., Hillsboro, OR, USA) scanning transmission electron microscope equipped with EDS (EDAX Inc., Mahwah, NJ, USA) using a CCD camera and Digital Micrograph Software (Gatan, Pleasanton, CA, USA). For the TEM analysis, a small amount of HAp/copolypetide hydrogel sample was placed onto a Formvar-coated copper grid and a negative contrast stain consisting of $1.0 \%$ ammonium molybdate at $\mathrm{pH} \sim 8$ was applied. After applying the stain, the excess solution was wicked away and the sample was allowed to dry.

Small angle X-ray scattering (SAXS). The structural order and length scale of the polymer gel structure were estimated by SAXS. Scattering measurements were performed at the 12ID beam line at the Advanced Photon Source at Argonne National Laboratory. Gel nanocomposite samples were sandwiched between Kapton tapes, and placed $2 \mathrm{~m}$ from the detector to investigate the nanocomposite structure and templating properties of the polypeptide. A $15 \times 15 \mathrm{~cm}$ detector was used to measure the scattered intensity and the transmitted intensity was measured using a photodiode. The beam energy used for the 
analysis is $12 \mathrm{keV}(\lambda=1.035 \AA)$ and the data were collected at $1 \mathrm{~s}$ exposure, and the collected 2D data were corrected, azimuthally averaged.

\section{Conclusions}

HAp/copolypeptide nanocomposites were obtained via a simple co-precipitation method at room temperature with the block copolypeptides serving as the organic template, similar to natural bone that is a complex inorganic (HAp) -organic (collagen) nanocomposite material. This method allows for simultaneous formation of the self-assembled block copolypeptide gel and of the inorganic phase, thereby enabling formation of a self-assembled nanocomposite. Block copolypeptide templates that form gels at very low weight fractions (0.5-4 wt\%) compared to previously investigated synthetic block copolymers, allow for inorganic content in the nanocomposites of over $50 \mathrm{wt} \%$, more closely approaching the inorganic content seen in natural bone. Previous research ${ }^{75,76}$ has shown that the increase of HAp content will result in an increase in the elastic modulus but a decrease in the tensile strength and ductility. A good compromise of these mechanical properties can be obtained in the composite of HAp weight fraction of 60-70\%, i.e. the composition of native bone. As mentioned earlier, most of the synthetic biomineralization efforts have yielded only about 30 $\mathrm{wt} \%$ inorganic fraction. Therefore, the present work is unique in that we have been able to increase the inorganic weight fraction to over 50\%, more closely approaching the inorganic content in bone. Solid state NMR studies proved the existence of a nanocomposite, with the inorganic phase composed mainly of hydroxyapatite but also $\mathrm{HPO}_{4}^{2-}$. The morphology of the inorganic nanoparticles is very different from that obtained by precipitation in the absence of the block copolypeptides. The data obtained from XRD, FTIR, S/TEM, SAED, HRTEM, EDS, solid state NMR and small angle scattering studies are consistent and lead us to the conclusion that the inorganic phase present in the nanocomposite is carbonated HAp of nanoscale dimensions, with elongated plate-like morphology very similar to the mineral phase of bone. Overall, this approach allows us to synthesize self-assembled calcium phosphate nanocomposites using organic templates, which could have a potential applications in soft tissue repair. 


\section{Acknowledgements}

This work was supported by the US Department of Energy under contract number DE-AC02-07CH11358. This work benefited from the use of APS and the IPNS, funded by the US DOE, Office of Science, Office of Basic Energy Science under contract no DEAC02-06CH11357. Supporting Information is available online from The Royal Society of Chemistry or from the authors.

\section{References}

1. S. V. Dorozhkin and M. Epple, Angewandte Chemie, International Edition, 2002, 41, 3130-3146.

2. R. K. Roeder, G. L. Converse, R. J. Kane and W. Yue, Jom, 2008, 60, 38-45.

3. M. Vallet-Regi, Dalton Transactions, 2006, 5211-5220.

4. A. Bigi, E. Boanini, S. Panzavolta, N. Roveri and K. Rubini, Journal of Biomedical Materials Research, 2002, 59, 709-715.

5. J. Y. Rho, L. Kuhn-Spearing and P. Zioupos, Med Eng Phys FIELD Full Journal Title:Medical engineering \& physics, 1998, 20, 92-102.

6. M. Vallet-Regi and J. M. Gonzalez-Calbet, Progress in Solid State Chemistry, 2004, 32, 1-31.

7. K. S. Anseth, V. R. Shastri, C. T. Laurencin and R. Langer, Abstracts of Papers of the American Chemical Society, 1996, 211, 228.

8. Z. Li, Y. Li, A. Yang, X. Peng, X. Wang and Z. Xiang, Journal of Materials Science: Materials in Medicine, 2005, 16, 213-219.

9. S. Mann, Biomineralization: Principles and Concepts in Bioinorganic Materials Chemistry, 2001.

10. Z. Amjad, Calcium Phosphates In Biological And Industrial Systems, Kluwer Academic Publishers, Boston, 1998.

11. A. Sinha, S. Nayar, A. Agrawal, D. Bhattacharyya and P. Ramachandrarao, Journal of the American Ceramic Society, 2003, 86, 357-359.

12. J. Song, V. Malathong and C. R. Bertozzi, Journal of the American Chemical Society, 2005, 127, 3366-3372. 
13. N. Spanos, V. Deimede and P. G. Koutsoukos, Biomaterials, 2001, 23, 947-953.

14. T. Taguchi, A. Kishida and M. Akashi, Journal of Biomaterials Science, Polymer Edition, 1999, 10, 795-804.

15. W. Tjandra, P. Ravi, J. Yao and K. C. Tam, Nanotechnology, 2006, 17, 5988-5994.

16. Y. F. Zhao and J. Ma, Microporous and Mesoporous Materials, 2005, 87, 110-117.

17. N. Degirmenbasi, D. M. Kalyon and E. Birinci, Colloids and Surfaces, B: Biointerfaces, 2006, 48, 42-49.

18. L. F. Sukhodub, C. Moseke, L. B. Sukhodub, B. Sulkio-Cleff, V. Y. Maleev, M. A. Semenov, E. G. Bereznyak and T. V. Bolbukh, Journal of Molecular Structure, 2004, 704, 53-58.

19. M. Kikuchi, S. Itoh, S. Ichinose, K. Shinomiya and J. Tanaka, Biomaterials, 2001, 22, 1705-1711.

20. F. Z. Cui, Y. Wang, Q. Cai and W. Zhang, Journal of Materials Chemistry, 2008, 18, 3835-3840.

21. I. Manjubala, S. Scheler, J. Bossert and D. Jandt Klaus, Acta biomaterialia, 2006, 2, 75-84.

22. V. M. Rusu, C.-H. Ng, M. Wilke, B. Tiersch, P. Fratzl and M. G. Peter, Biomaterials, 2005, 26, 5414-5426.

23. L. Wang and C. Li, Carbohydrate Polymers, 2007, 68, 740-745.

24. S. A. Hutchens, R. S. Benson, B. R. Evans, H. M. O'Neill and C. J. Rawn, Biomaterials, 2006, 27, 4661-4670.

25. L. Hong, Y. L. Wang, S. R. Jia, Y. Huang, C. Gao and Y. Z. Wan, Materials Letters, 2006, 60, 1710-1713.

26. P. Cromme, C. Zollfrank, L. Mueller, F. A. Mueller and P. Greil, Materials Science \& Engineering, C: Biomimetic and Supramolecular Systems, 2007, 27, 1-7.

27. K. R. Mohamed and A. A. Mostafa, Materials Science \& Engineering, C: Materials for Biological Applications, 2008, 28, 1087-1099.

28. M. C. Chang, C.-C. Ko and W. H. Douglas, Biomaterials, 2003, 24, 3087-3094. 
29. D. Enlow, A. Rawal, M. Kanapathipillai, K. Schmidt-Rohr, S. Mallapragada, C. T. Lo, P. Thiyagarajan and M. Akinc, Journal of Materials Chemistry, 2007, 17, 15701578.

30. M. Kanapathipillai, Y. Yusufoglu, A. Rawal, Y. Y. Hu, C. T. Lo, P. Thiyagarajan, Y. E. Kalay, M. Akinc, S. Mallapragada and K. Schmidt-Rohr, Chemistry of Materials, 2008, ACS ASAP.

31. Y. Yusufoglu, Y. Y. Hu, M. Kanapathipillai, K. Matthew, Y. E. Kalay, P. Thiyagarajan, M. Akinc, K. Schmidt-Rohr and S. Mallapragada, J. Mater. Res, 2008.

32. L. Wang, Y. Li and C. Li, J. Nanopart. Res., 2009, 11, 691-699.

33. S. -C. Liou, S. - Y. Chen and D. -M. Liu, Biomaterials, 2003, 24, 3981-3988.

34. J. N. Cha, G. D. Stucky, D. E. Morse and T. J. Deming, Nature, 2000, 403, 289-292.

35. L. E. Euliss, S. G. Grancharov, S. O'Brien, T. J. Deming, G. D. Stucky, C. B. Murray and G. A. Held, Nano Lett., 2003, 3, 1489-1493.

36. V. Krikorian, M. Kurian, M. E. Glavin, A. P. Nowak, T. J. Deming and D. J. Pochan, Polym. Sci. Polym. Phys., 2002, 40, 2579-2586.

37. M. M. Tomczak, D. D. Glawe, L. F. Drummy, C. G. Lawrence, M. O. Stone, C. C. Perry, D. J. Pochan, T. J. Deming and R. R. Naik, J. Am. Chem. Soc., 2005, 127, 12577-12582.

38. K. Donadel, M. C. M. Laranjeira, V. L. Goncalves, V. T. Favere, J. C. de Lima and L. H. M. Prates, J. Am. Ceram. Soc., 2005, 88, 2230-2235.

39. K. Kandori, N. Horigami, A. Yasukawa and T. Ishikawa, J. Am. Ceram. Soc., 1997, 80, $1157-1164$.

40. A. Lopez-Macipe, J. Gomez-Morales and R. Rodriguez-Clemente, Adv. Mater., 1998, 10, 49-53.

41. Y. Yusufoglu and M. Akinc, Journal of the American Ceramic Society, 2008, 91, 7782.

42. M. Aizawa, A. E. Porter, S. M. Best and W. Bonfield, Biomaterials, 2005, 26, $3427-$ 3433.

43. M. Aizawa, H. Ueno, K. Itatani and I. Okada, J. Eur. Ceram. Soc., 2006, 26, 501-507. 
44. M. Kinoshita, K. Itatani, S. Nakamura and A. Kishioka, Gypsum Lime, 1990, 227, 207-215.

45. A. Barroug, J. Lemaitre and P. G. Rouxhet, J. Alloys. Compd., 1992, 188, 152-156.

46. K. Ishikawa, E. D. Eanes and M. S. Tung, J. Dent. Res., 1994, 73, 1462-1469.

47. A. Slosarczyk, Z. Paszkiewicz and C. Paluszkiewicz, J. Mol. Struct., 2005, 744-747, 657-661.

48. T. Iizuka and A. Nozuma, J. Ceram. Soc. Japan, 1998, 106, 820-823.

49. R. Z. LeGeros, J. P. LeGeros, O. R. Trautz and W. P. Shirra, Adv. X-Ray Anal., 1971, 14, 57-66.

50. E. A. P. De Maeyer, R. M. H. Verbeeck and D. E. Naessens, Inorg. Chem., 1993, 32, 5709-5714.

51. J. Liu, X. Ye, H. Wang, M. Zhu, B. Wang and H. Yan, Ceram. Int., 2003, 29, 629633.

52. H. Monma and T. Kamiya, J. Mater. Sci., 1987, 22, 4247-4250.

53. J. C. Elliott, Structure and Chemistry of the Apatites and Other Calcium Orthophosphates; Elsevier: Amsterdam, 1994.

54. S. N. Danilchenko, C. Moseke, L. F. Sukhodub and B. Sulkio-Cleff, Crystal Research and Technology, 2004, 39, 71-77.

55. S. Liao, F. Watari, M. Uo, S. Ohkawa, K. Tamura, W. Wang and F. Cui, Journal of Biomedical Materials Research, Part B: Applied Biomaterials, 2005, 74B, 817-821.

56 J. P. Coates, Appl. Spectrosc. Rev., 1996, 31, 179-192.

57. G. Xu, I. A. Aksay and J. T. Groves, Journal of the American Chemical Society, 2001, 123, 2196-2203.

58. K. Sato, Topics in Current Chemistry, 2007, 270, 127-153.

59. R. Murugan, S. Ramakrishna and K. P. Rao, Materials Letters, 2006, 60, 2844-2847.

60. $\quad$ E. Sachlos, D. Gotora and J. T. Czernuszka, Tissue Engineering, 2006, 12, 24792487.

61. N. A. Zakharov, Z. A. Ezhova, E. M. Koval, V. T. Kalinnikov and A. E. Chalykh, Inorganic Materials, 2005, 41, 509-515. 
62. W. P. Rothwell, J. S. Waugh and J. P. Yesinowski, Journal of the American Chemical Society, 1980, 102, 2637-2643.

63. S. S. Hou, F. L. Beyer and K. Schmidt-Rohr, Solid State Nuclear Magnetic Resonance, 2002, 22, 110-127.

64. K. Wuthrich, NMR of Proteins and Nucleic Acids, 1986.

65. Q. Chen, S. S. Hou and K. Schmidt-Rohr, Solid State Nuclear Magnetic Resonance, 2004, 26, 11-15.

66. Y. Wang, Journal of Biomolecular NMR, 2004, 30, 233-244.

67. C. Ye, R. Fu, J. Hu, L. Hou and S. Ding, Magnetic Resonance in Chemistry, 1993, 31, 699-704.

68. A. P. Nowak, V. Breedveld, L. Pakstis, B. Ozbas, D. J. Pine, D. Pochan and T. J. Deming, Nature (London, United Kingdom), 2002, 417, 424-428.

69. X. Su, K. Sun, F. Z. Cui and W. J. Landis, Bone (New York, NY, United States), 2003, 32, 150-162.

70. P. Houlle, J. C. Voegel, P. Schultz, P. Steuer and F. J. G. Cuisinier, Journal of Dental Research, 1997, 76, 895-904.

71. W. J. Landis, M. J. Song, A. Leith, L. McEwen and B. F. McEwen, Journal of structural biology, 1993, 110, 39-54.

72. P. Fratzl, N. Fratzl-Zelma, K. Klaushofer, G. Vogl and K. Koller, Calcif. Tissue Int., 1991, 48, 407-413.

73. P. Fratzl, M. Groschner, G. Vogl, H. Plenk, J. Eschberger, N. Fratzl-Zelman, K. Koller and K. Klaushofer, J. Bone Mineral Res., 1992, 7, 329-334.

74. K. Schmidt-Rohr, J. Clauss and H. W. Spiess, Macromolecules, 1992, 25, 3273-3277.

75 L. Fang, L. Yang and P. Gao, Key Eng. Mater., 2007, 10, 334-335.

76 S. Mollazadeh, J. Javadpour and A. Khavandi, Adv. Appl. Ceram., 2007, 106, 165170.

77. R. Gonzalez-McQuire, J.-Y. Chane-Ching, E. Vignaud, A. Lebugle and S. Mann, Journal of Materials Chemistry, 2004, 14, 2277-2281.

78. J. D. Hartgerink, E. Beniash and S. I. Stupp, Science (Washington, DC, United States), 2001, 294, 1684-1688. 
79. A. D. Roddick-Lanzilotta and A. J. McQuillan, Journal of Colloid and Interface Science, 1999, 217, 194-202.

80. M. Rozenberg and G. Shoham, Biophysical Chemistry, 2007, 125, 166-171.

\section{Tables and Figures}

Table 4.1 The possible assignments of FTIP peaks present in Figure 4.2 (ref. 23, 56, 60, 61, 77-80).

\begin{tabular}{|c|c|}
\hline Group Frequency $\left(\mathrm{cm}^{-1}\right)$ & Assignment \\
\hline 3570 & Hydroxyl stretching \\
\hline $3450($ broad) $/ 1640$ & Water absorption bands \\
\hline 3350 & $\mathrm{~N}-\mathrm{H}$ stretching of amine groups \\
\hline 3280 & $\mathrm{~N}-\mathrm{H}$ stretching of amide $\mathrm{I}$ \\
\hline $2970 / 2850$ & $\mathrm{C}-\mathrm{H}$ stretching \\
\hline 2050 & $-\mathrm{NH}_{3}{ }^{+}$bending mode \\
\hline 1630 & $\begin{array}{l}\text { Amide I mainly CO group } \\
\text { stretching mode }\end{array}$ \\
\hline 1537 & $\begin{array}{l}\text { Amide II mainly } \mathrm{N}-\mathrm{H} \text { bend and } \mathrm{C}- \\
\mathrm{N} \text { stretch }\end{array}$ \\
\hline $1470 / 730$ & $\begin{array}{l}\mathrm{C}-\mathrm{H} \text { bending and methylene } \\
\text { rocking }\end{array}$ \\
\hline 1390 & Methyl $\mathrm{C}-\mathrm{H}$ bend \\
\hline $1380 / 832$ & Nitrate groups \\
\hline 1350 & Methyne $\mathrm{C}-\mathrm{H}$ vibrations \\
\hline 1300 & $\mathrm{CH}_{2}$ deformation and wagging \\
\hline 1250 & $\begin{array}{l}\text { Amide III mainly } \mathrm{C}-\mathrm{N} \text { stretch and } \\
\mathrm{N}-\mathrm{H} \text { in-plane deformation }\end{array}$ \\
\hline 1160 & $\mathrm{C}-\mathrm{N}$ stretch of amine groups \\
\hline $1080 / 700$ & $\mathrm{C}-\mathrm{C}$ vibrations \\
\hline $873 / 1415 / 1462$ & $\begin{array}{l}\text { Carbonate } v_{2} / v_{3 \mathrm{a}} / v_{3 \mathrm{~b}} \text { (for B- } \\
\mathrm{CO}_{3} \mathrm{HAp} \text { ) }\end{array}$ \\
\hline $566-601 / 962 / 1040-1100$ & Phosphate bands of HAp \\
\hline
\end{tabular}




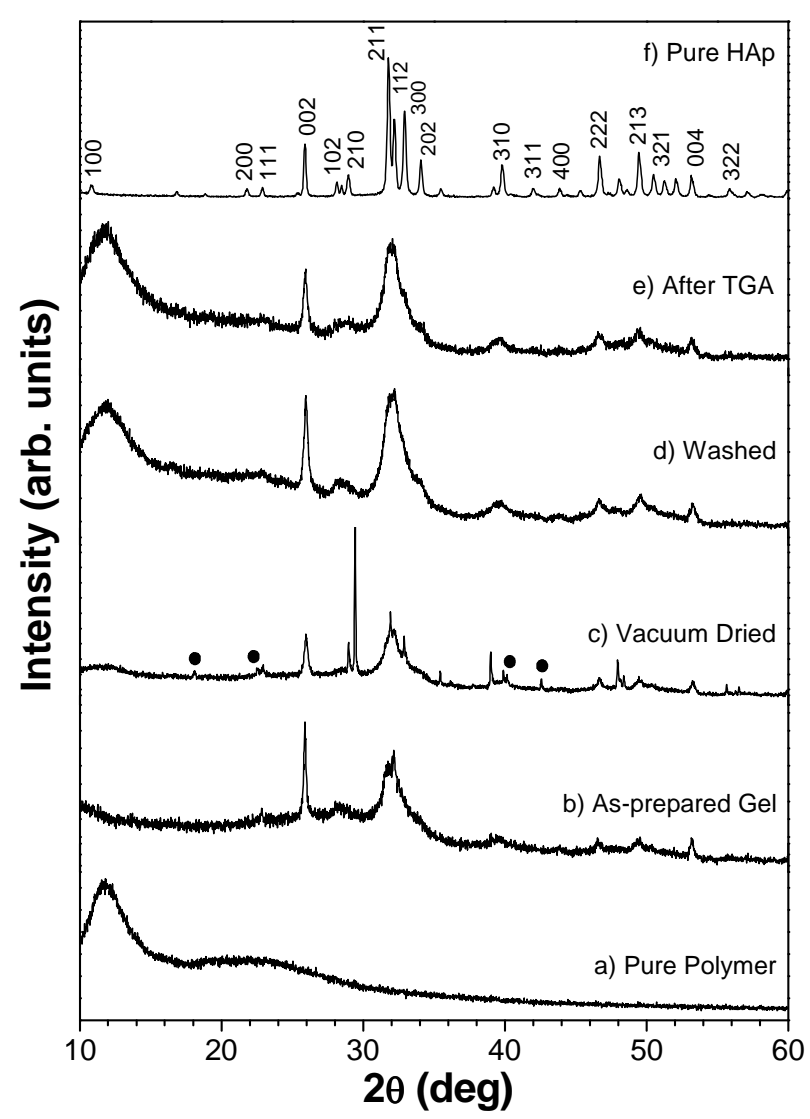

Figure 4.1. X-ray diffraction patterns of a) pure polymer, b) as-prepared calcium phosphate/hydrogel nanocomposite, c) vacuum dried nanocomposite, d) washed sample (after vacuum drying), e) calcined sample after TGA experiments and f) pure HAp (Standard Reference Material ${ }^{\circledR}$ 2910). Data were collected by Y. Yusufgolu. 


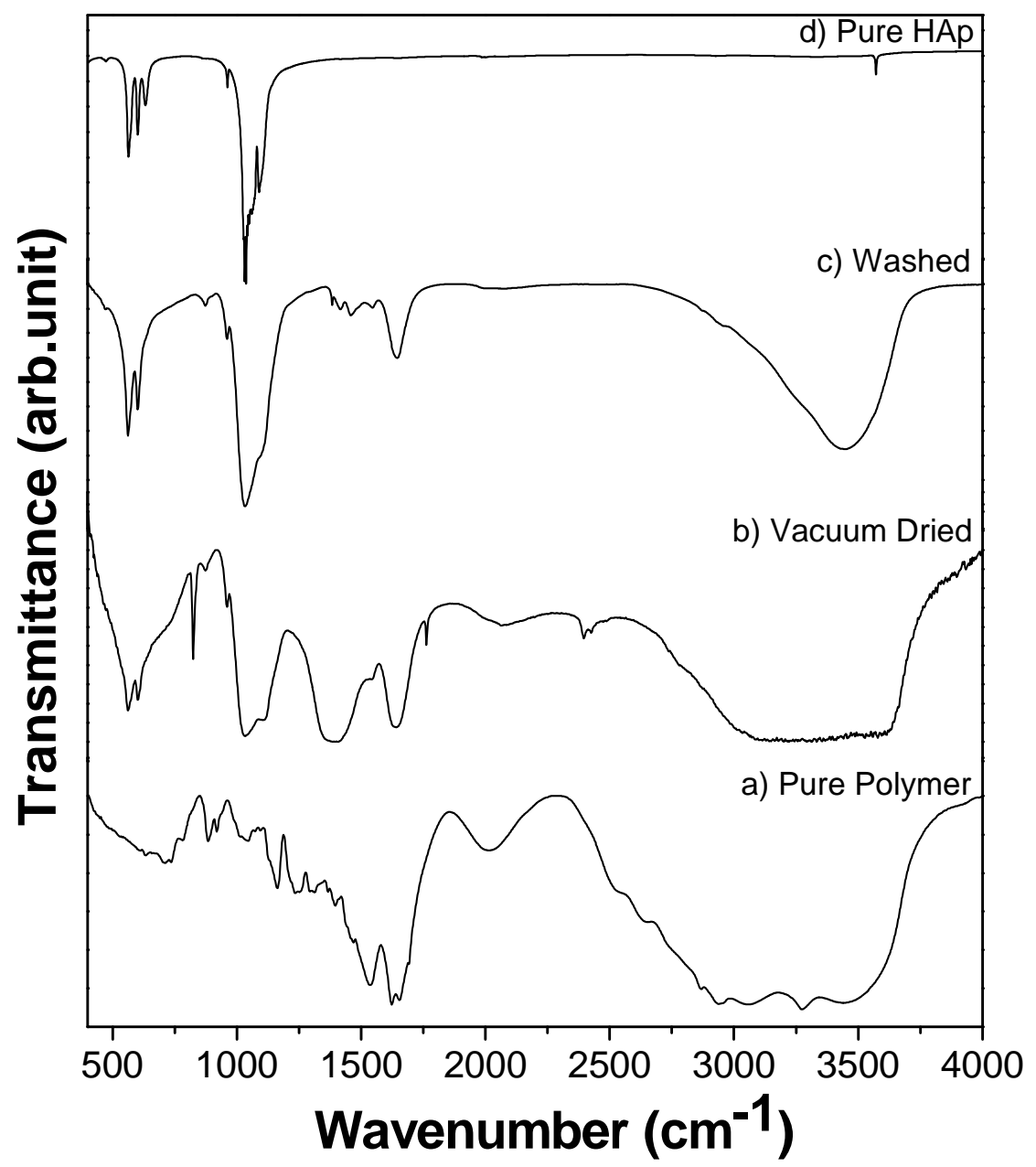

Figure 4.2. FTIR spectra of the a) pure polymer, b) vacuum dried HAp/polypeptide hydrogel nanocomposite, c) washed sample (after vacuum drying), and d) pure HAp (Standard Reference Material $^{\circledR}$ 2910). Data were collected by Y. Yusufgolu. 


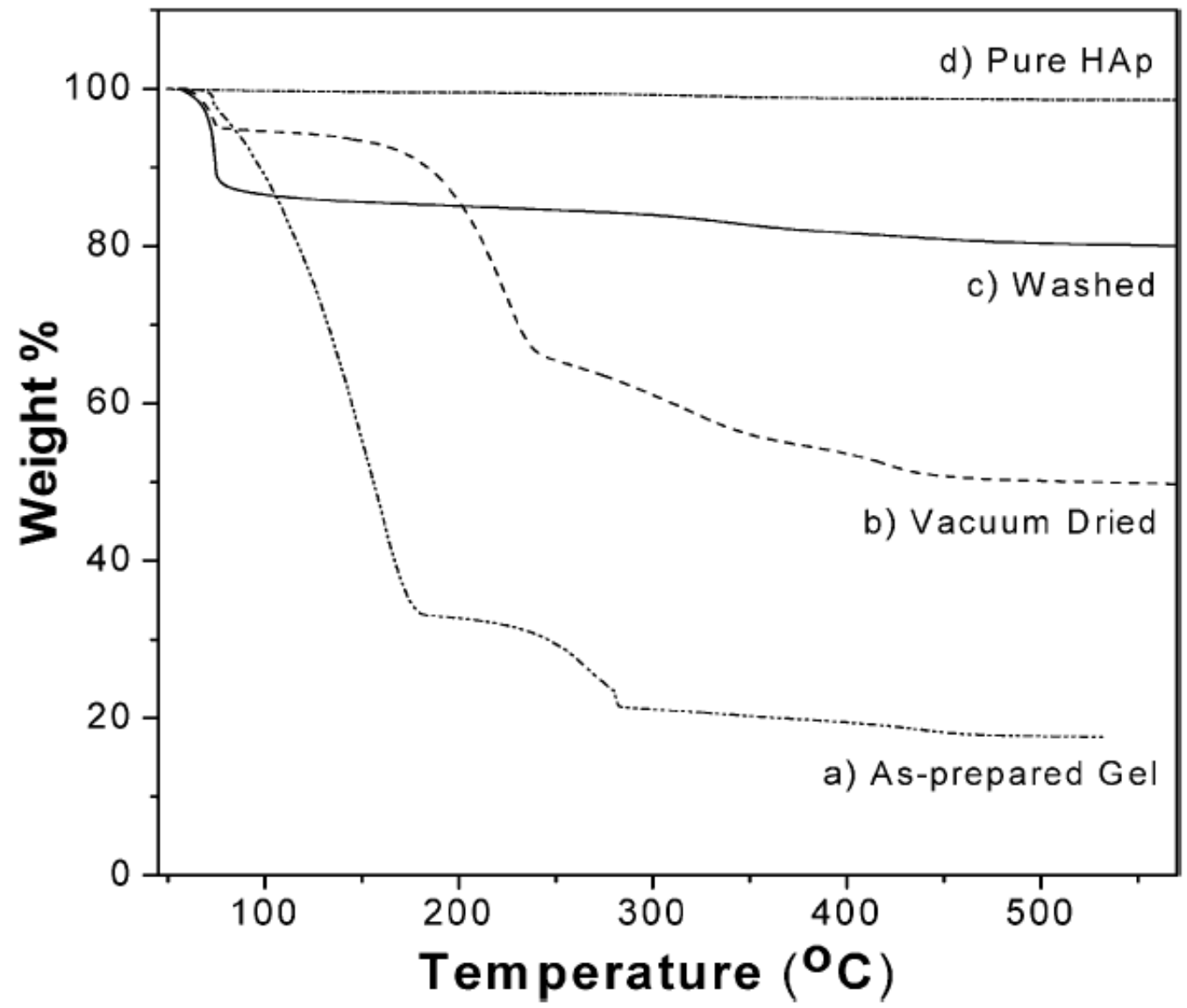

Figure 4.3. TGA thermographs of a) as-prepared HAp/polypeptide hydrogel, b) vacuum dried HAp/polypeptide nanocomposite, c) washed HAp/polypeptide nanocomposite and d) pure HAp (Standard Reference Material® 2910). Data were collected by M. Kanapathipillai. 


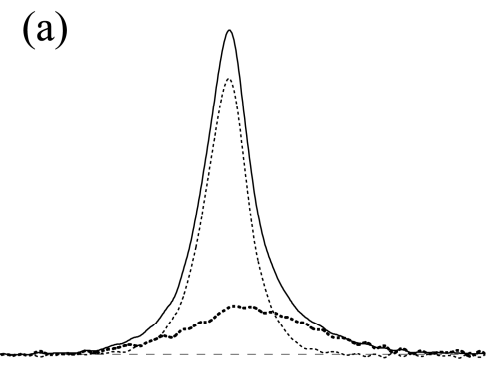

$\begin{array}{lllllllll}10 & 8 & 6 & 4 & 2 & 0 & -2 & -4 & \text { ppm }\end{array}$ (b)

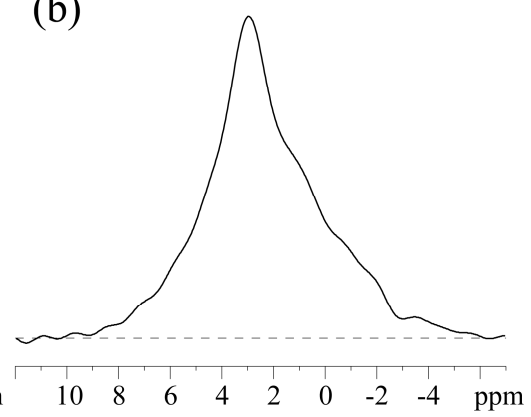

Figure 4.4. ${ }^{31} \mathrm{P}$ NMR spectra of the synthesized composite. (a) Solid line: DP/MAS spectrum of all phosphates. Deconvolution based on ${ }^{1} \mathrm{H}_{-}{ }^{31} \mathrm{P}$ dipolar dephasing is also shown; thin dashed line: $\mathrm{PO}_{4}{ }^{3-}, 68 \%$; thick dashed line: protonated or $\mathrm{H}$-bonded phosphate, $32 \%$. (b) CP/MAS with 1-ms cross polarization time.
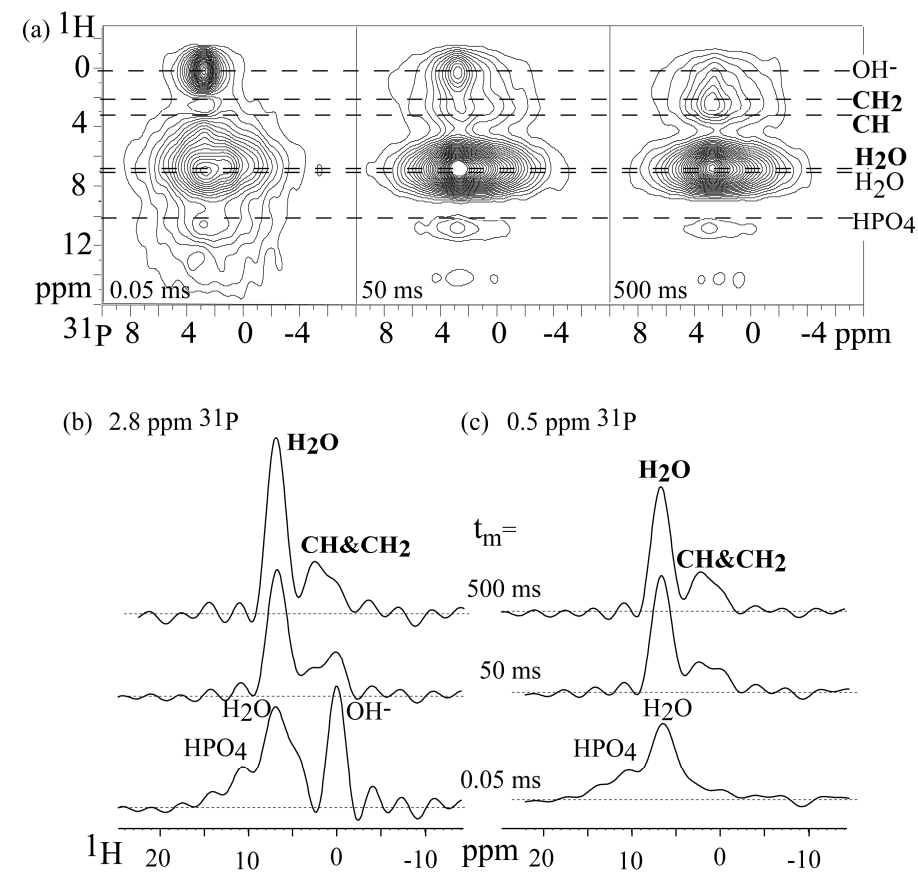

Figure 4.5. (a) $2 \mathrm{D}^{1} \mathrm{H}^{31} \mathrm{P}$ HETCOR NMR spectra of the synthesized composite, with spin diffusion mixing times of $0.05 \mathrm{~ms}, 50 \mathrm{~ms}$, and $500 \mathrm{~ms}$. (b) and (c): Cross sections of the 2D spectra at $2.8 \mathrm{ppm}$ and $0.5 \mathrm{ppm}{ }^{31} \mathrm{P}$, respectively. 

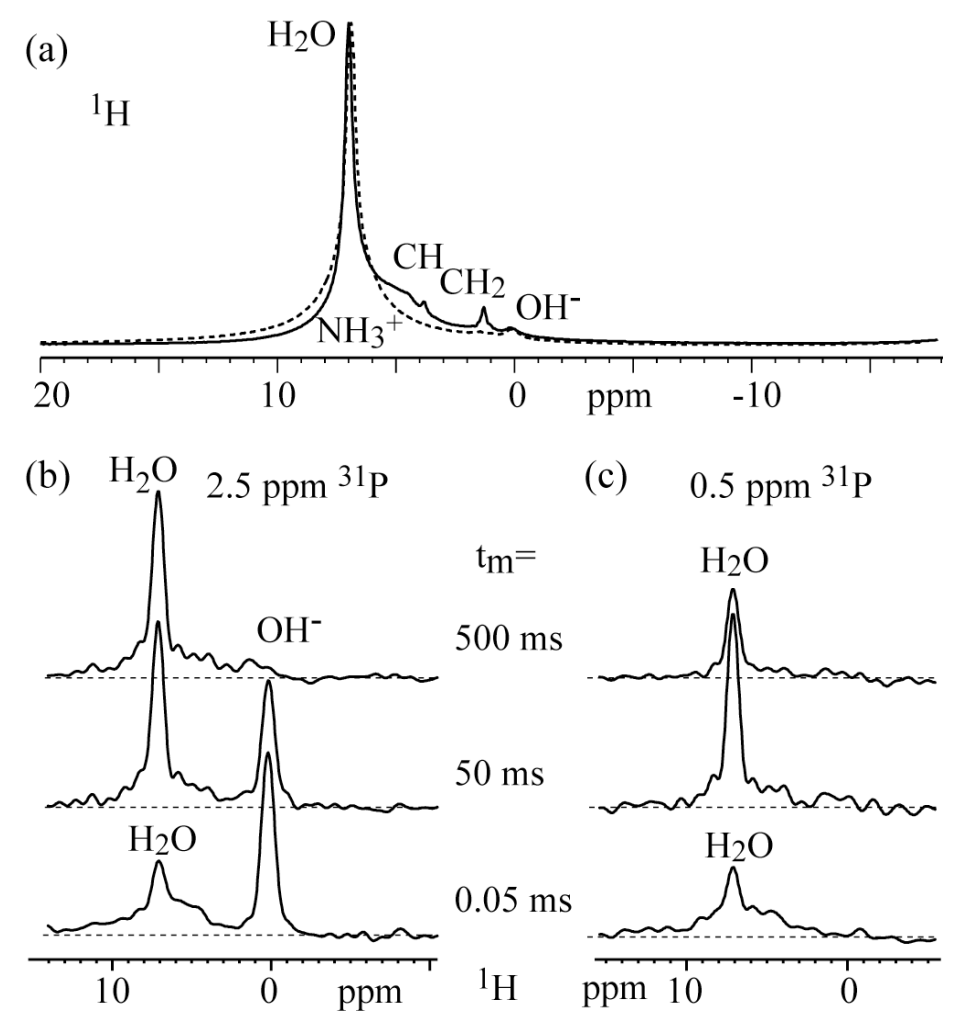

Figure 4.6. ${ }^{1} \mathrm{H}$ spectra of the synthesized composite. (a) One-pulse ${ }^{1} \mathrm{H}$ spectrum of regular vacuum dried sample (solid line) and intensively dried sample (dashed line). (b, c) Cross sections from 2D "WISE" spectra at $2.5 \mathrm{ppm}$ and $0.5 \mathrm{ppm}{ }^{31} \mathrm{P}$, respectively, for a series of spin-diffusion times.

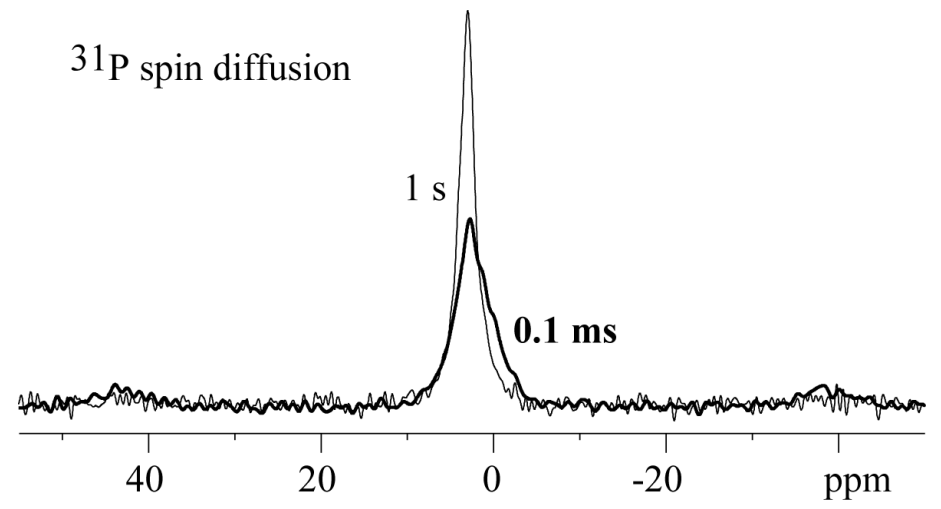

Figure 4.7. ${ }^{31} \mathrm{P}$ NMR of the nanocomposite, with ${ }^{31} \mathrm{P}$ spin diffusion for $0.1 \mathrm{~ms}$ (thick line) and $1 \mathrm{~s}$ (thin line) after short $(0.1-\mathrm{ms})$ cross polarization that selectively polarizes the protonated phosphates. MAS frequency: $6.5 \mathrm{kHz}$. 


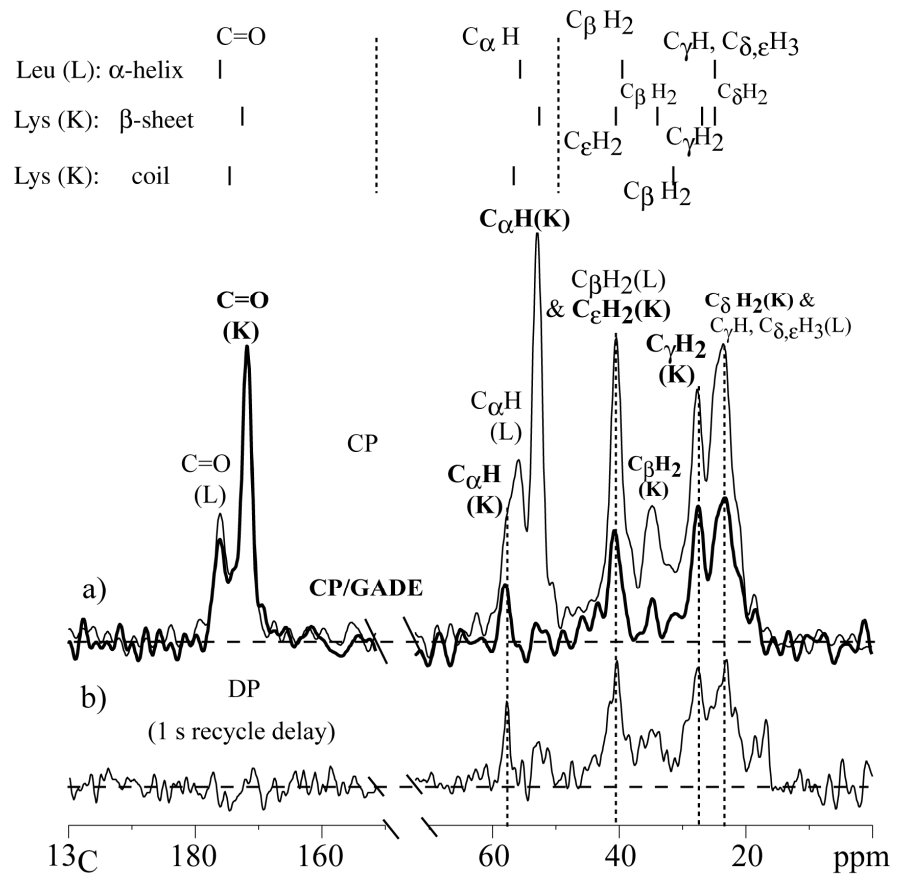

Figure 4.8. Solid-state ${ }^{13} \mathrm{C}$ NMR spectra of $\mathrm{K}_{170} \mathrm{~L}_{30}$ in the nanocomposite. a) $\mathrm{CP}$ (thin line) and $\mathrm{CP}$ with $40 \mu$ s gated decoupling (thick line). b) direct polarization (DP) with $1 \mathrm{~s}$ recycle delay. Literature values of the solid-state ${ }^{13} \mathrm{C}$ chemical shifts are listed above the spectra for different peptide secondary structures. 


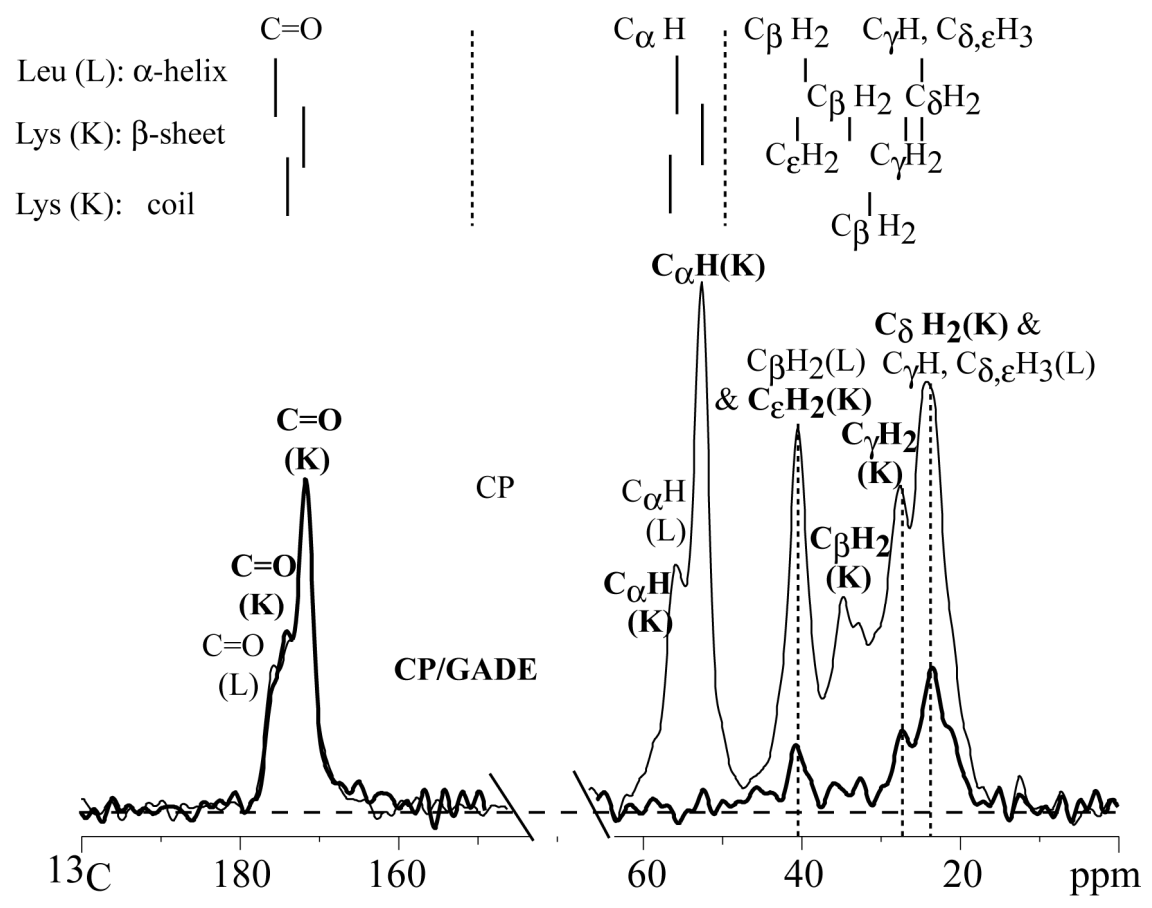

Figure 4.9. Solid-state ${ }^{13} \mathrm{C}$ NMR spectra of the nanocomposite after intensive drying. CP (thin line) and CP with $40 \mu$ s gated decoupling (thick line). 

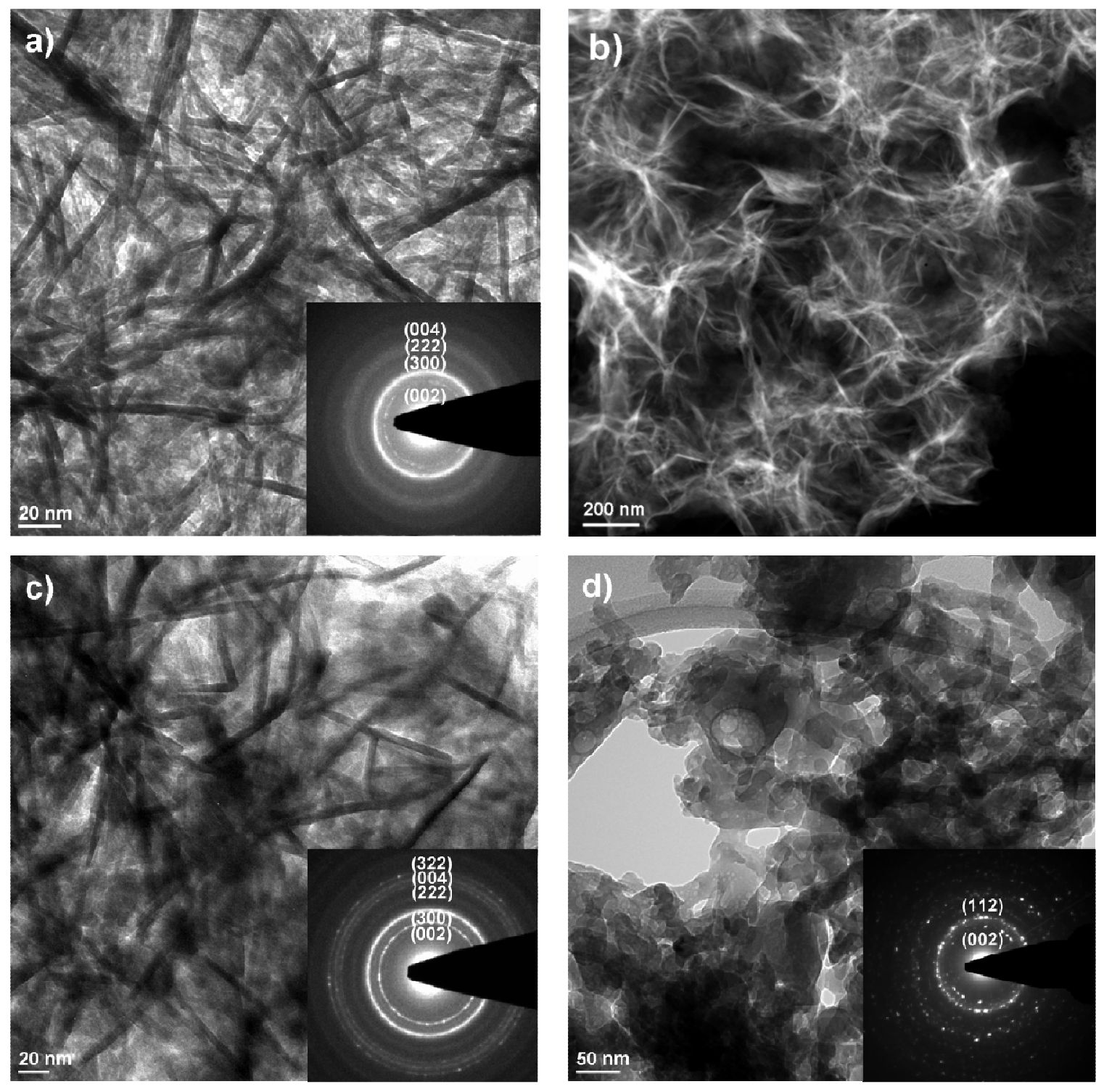

Figure 4.10. TEM images and corresponding SAED patterns of HAp/polypeptide nanocomposite: a) bright field, b) STEM/HAADF, c) washed HAp/polypeptide nanocomposite (after vacuum drying), d) HAp nanocrystals obtained by direct synthesis from an aqueous solution in the absence of polymer. Data were collected by Y. Yusufgolu. 

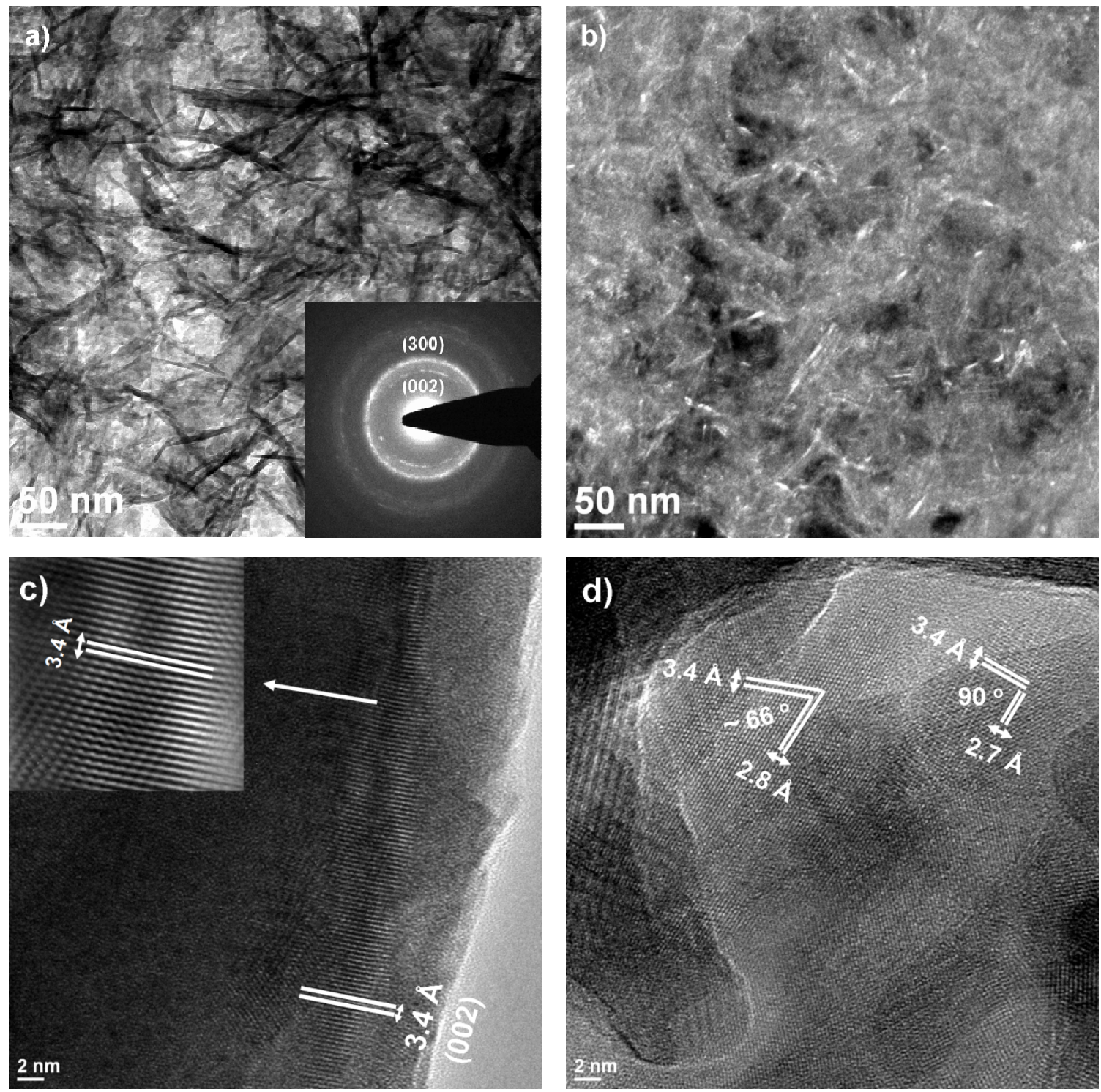

Figure 4.11. a) BF-TEM and b) corresponding DF-TEM images of HAp/polypeptide nanocomposite, c-d) HRTEM images of HAp/polypeptide nanocomposite. The lower inset in Fig. 4.11a shows the corresponding SEAD pattern and the upper inset in Fig. 4.11c shows the filtered inverse-FFT image of the indicated area. Data were collected by Y. Yusufgolu. 


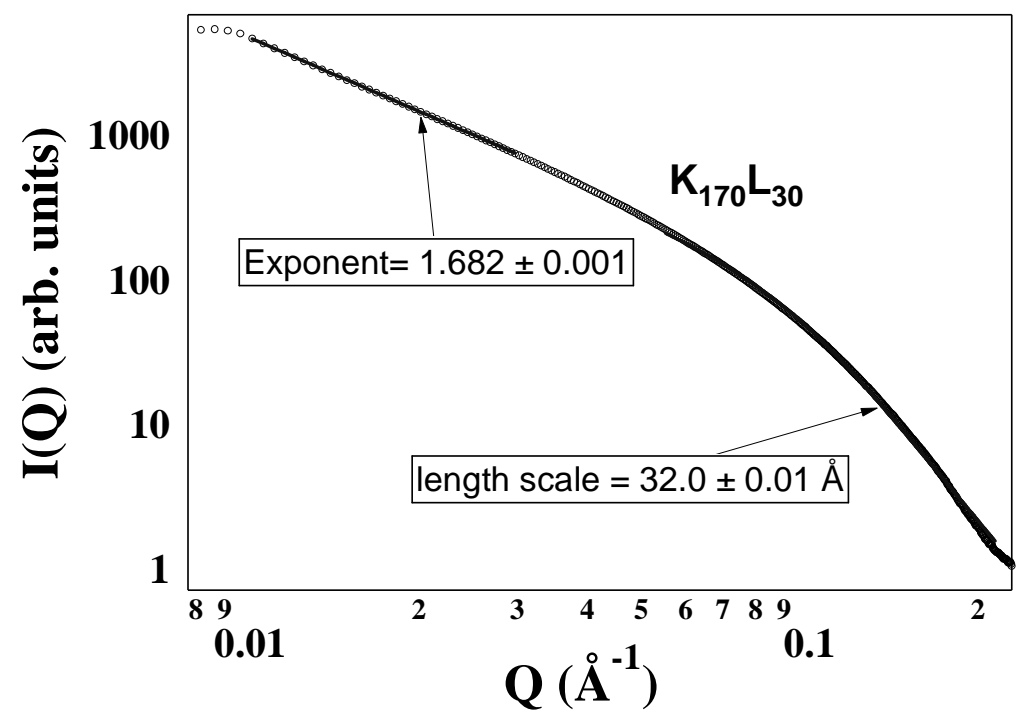

Figure 4.12: SAXS of polypeptide nanocomposite. SAXS data were fitted using a combined equation consisting of an exponential and a power-law for the high Q region and power-law fitting was used for the low Q region. Data were collected by M. Kanapathipillai 


\section{Supporting Information for}

Self-assembled calcium phosphate nanocomposites using block copolypeptide templates Yanyan $\mathrm{Hu}$, Yusuf Yusufoglu, Mathumai Kanapathipillai, Chu-Ya Yang, YaQiao Wu, Papannan Thiyagarajan, Timothy Deming, Mufit Akinc, Klaus Schmidt-Rohr,

\section{Surya Mallapragada}

(a)

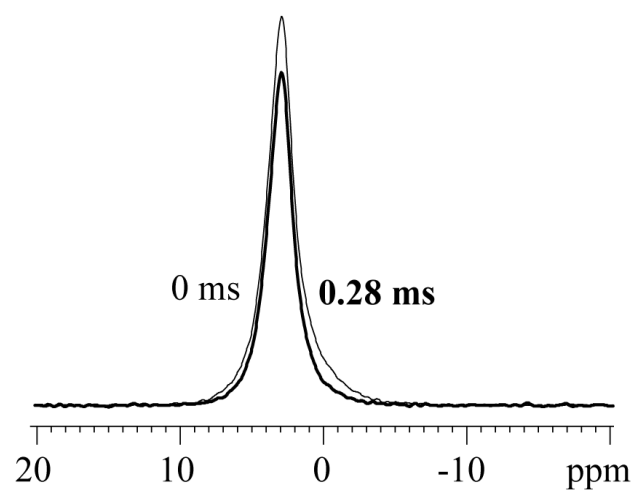

(b)

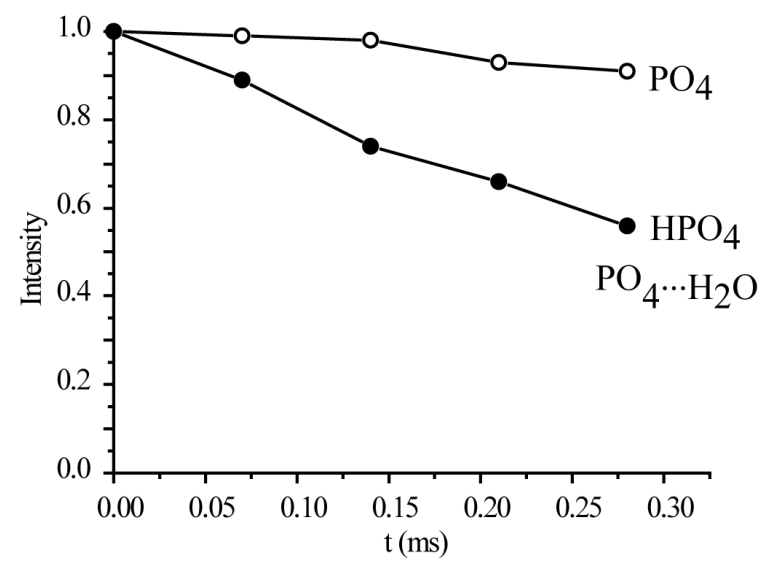

Figure 4.S1. Determination of phosphate protonation state by ${ }^{31} \mathrm{P}$ NMR. (a) DP/MAS with recoupled ${ }^{1} \mathrm{H}_{-}{ }^{31} \mathrm{P}$ dipolar dephasing for zero (thin line) and two rotation periods (thick line). (b) Plot of normalized ${ }^{31} \mathrm{P}$ NMR intensity as a function of dipolar dephasing time. 


\title{
Chapter 5
}

\section{Broadband "Infinite-Speed" Magic-Angle Spinning NMR Spectroscopy}

A paper published in Journal of the American Chemical Society

2009, 131, 8390-8391

Yan-Yan $\mathrm{Hu}^{\mathrm{a}}$, E. M. Levin ${ }^{\mathrm{b}}$, Klaus Schmidt-Rohr ${ }^{\mathrm{a}}$

${ }^{a}$ Ames Laboratory and Department of Chemistry, Iowa State University, Ames, IA 50011, USA

${ }^{\mathrm{b}}$ Ames Laboratory and Department of Physics and Astronomy, Iowa State University, Ames, IA 50011, USA

\begin{abstract}
High-resolution magic-angle spinning NMR of high-Z spin- $1 / 2$ nuclei such as ${ }^{125} \mathrm{Te}$, ${ }^{207} \mathrm{~Pb},{ }^{119} \mathrm{Sn},{ }^{113} \mathrm{Cd}$, and ${ }^{195} \mathrm{Pt}$ is often hampered by large (>1,000 ppm) chemical-shift anisotropies, which result in strong spinning sidebands that can obscure the centerbands of interest. In various tellurides with applications as thermoelectrics and as phase-change materials for data storage, even $22-\mathrm{kHz}$ magic-angle spinning cannot resolve the center- and sidebands broadened by chemical-shift dispersion, which precludes peak identification or quantification. For sideband suppression over the necessary wide spectral range (up to 200 $\mathrm{kHz}$ ), radio-frequency pulse sequences with few, short pulses are required. We have identified Gan's two-dimensional magic-angle-turning (MAT) experiment with five $90^{\circ}$ pulses as a promising broadband technique for obtaining spectra without sidebands. We have adapted it to broad spectra and fast magic-angle spinning by accounting for long pulses (comparable to the dwell time in $\mathrm{t}_{1}$ ) and short rotation periods. Spectral distortions are small and residual sidebands negligible even for spectra with signals covering a range of $1.5 \gamma \mathrm{B}_{1}$, due to a favorable disposition of the narrow ranges containing the signals of interest in the spectral plane. The method is demonstrated on various technologically interesting tellurides with spectra spanning up to $170 \mathrm{kHz}$, at $22 \mathrm{kHz}$ MAS.
\end{abstract}

\section{Main text}

Determination of the spectrum of isotropic spectral shifts (chemical, Knight, or paramagnetic shifts) is a central goal of high-resolution NMR. For spin-1/2 isotopes, magic- 
angle spinning (MAS) can often achieve this goal, but in some cases spinning sidebands, due to large chemical-shift anisotropies, may obscure the isotropic-shift information. This is a particularly serious problem for high-Z nuclei, such as ${ }^{125} \mathrm{Te},{ }^{207} \mathrm{~Pb},{ }^{119} \mathrm{Sn},{ }^{113} \mathrm{Cd}$, and ${ }^{195} \mathrm{Pt}$, which have large chemical-shift anisotropies when they are located in low-symmetry (less than cubic) environments. In some cases, even at relatively high-speed MAS the sidebands are still merged; Figure 5.1 shows that this problem exists in $22-\mathrm{kHz}$ MAS ${ }^{125} \mathrm{Te}$ NMR spectra of various tellurides, several of which have interesting applications as thermoelectrics $^{1,2}$ or as phase-change materials for reversible data storage on CDs and DVDs $^{3}$. While the sidebands in the spectrum of $\mathrm{Ag}_{2}$ Te, Figure 5.1a, are narrow enough to be merely a nuisance, those of $\mathrm{Sb}_{2} \mathrm{Te}_{3}$ and GeTe, see Figures 5.1b and c, respectively, are merged due to a broad distribution of isotropic shifts, whose spectrum cannot be determined under these conditions. In the high-performance thermoelectric $\mathrm{Ag}_{0.53} \mathrm{~Pb}_{18} \mathrm{Sb}_{1.2} \mathrm{Te}_{20}{ }^{2}$, Figure $5.1 \mathrm{~d}$, the relative intensity of the smaller band of Te bonded to $\mathrm{Sb}$ at $-1500 \mathrm{ppm}$ cannot be reliably determined because of overlap from (unresolved) sidebands of the main peak. It appears that similar effects, manifested as no line narrowing in $8-\mathrm{kHz}$ MAS ${ }^{125} \mathrm{Te}$ NMR, were also observed for some transition-metal tellurides. ${ }^{4}$

Faster magic-angle spinning can increase the spacing of sidebands and decrease their intensities. Nevertheless, an often superior approach is complete removal of spinning sidebands by suitable sequences of short radio-frequency pulses ${ }^{5-7}$ producing a large nutation frequency $\gamma B_{1}$, since in most NMR probeheads the maximum $\gamma B_{1}$ clearly exceeds the maximum MAS frequency $\omega_{\mathrm{r}}$ achievable. For instance, in our probehead with 2.5-mm rotors, $\omega_{\mathrm{r}}<2 \pi 35 \mathrm{kHz}$, while $\gamma \mathrm{B}_{1}>2 \pi 110 \mathrm{kHz}$.

Various pulse sequences with four or more $180^{\circ}$ pulses have been introduced for sideband suppression, ${ }^{5-7}$ but due to the length and unfavorable off-resonance properties of $180^{\circ}$ pulses, they cannot achieve the broadband excitation required for spectra as in Figure 5.1 spanning $100 \mathrm{kHz}$ or more. In particular, total suppression of sidebands (TOSS) ${ }^{5}$ does not work well in many of these cases, giving very small intensities for sites with large sidebands. The projection of suitably sheared phase alternated spinning sideband (PASS) NMR spectra, ${ }^{6}$ obtained with five $180^{\circ}$ pulses, can provide quantitative spectra with only minor sidebands and with good sensitivity, but only for moderate ranges in isotropic chemi- 
cal shifts $(<0.7 \gamma \mathrm{B} 1)^{7}$ Instead, we have identified Gan's magic-angle turning (MAT) experiment ${ }^{8,9}$ with five $90^{\circ}$ pulses as a superior alternative; here, the projection after suitable shearing yields the "infinite-speed" spectrum free of spinning sidebands. The MAT experiment provides a larger bandwidth than TOSS or PASS since it requires only four $\sim 90^{\circ}$ pulses in addition to the regular excitation pulse, see Figure 5.2a. The bandwidth of a 90o pulse is large, $>5 \gamma \mathrm{B} 1$, if the length of the magnetization in the transverse plane is considered, but one needs to account for the nearly linear off-resonance phase shift $\approx$ $\omega 1 \mathrm{tp} / 2 .^{10}$ Thus, evolution occurs effectively for about half the pulse duration $t_{p}$, as indicated in Figure 5.2. As a result, a nominal evolution time $t_{1, \text { nom }} / 2=0$ corresponds to an effective evolution time $t_{1} / 2 \approx t_{p}$, see Figure 5.2b. The first slice without evolution, $t 1 / 2=0$, is obtained by omitting the "evolution pulses" altogether, see Figure 5.2c.

While MAT has mostly been applied at low spinning frequencies of $\sim 0.1 \mathrm{kHz}$ to measure site-resolved chemical-shift anisotropies, ${ }^{8,9}$ for our purpose fast spinning with frequencies $>20 \mathrm{kHz}$ is required to maximize sensitivity, using the MAT \pm approach $^{9}$ for solving the $2 \mathrm{D}$ phase problem. After shearing by $\arctan (1 / 2)$ along $\omega_{1}$, by $\arctan (-2 / 3)$ along $\omega_{2}$, and scaling of $\omega_{1}$ ' by $2 / 3$, the signal is concentrated in narrow vertical ridges (centerband and spinning sidebands), see Figure 5.3a. Summation of vertical slices at these ridges (rather than over a wide range of noise or potential artifacts) gives the desired spectrum without sidebands. Cyclic decrementation of $\mathrm{t}_{\mathrm{zA}}$ as indicated in Figure 5.2a keeps $\mathrm{t}_{\mathrm{zA}}$ and $\mathrm{t}_{\mathrm{zB}}$ short, avoiding $\mathrm{T}_{1}$ relaxation and loss of synchronization. Since the time signal of a broad spectrum decays fast, we have added a rotation-synchronized Hahn spin echo before detection, which moves the signal out of the pulse dead time without interfering with rotary refocusing. Compared to a regular 1D spectrum, sensitivity in MAT is reduced by a factor of $\sim 1 / 4$, because during $\mathrm{t}_{\mathrm{zA}}$ and $\mathrm{t}_{\mathrm{zB}}$ only one magnetization component is retained.

Figure 5.3a shows a fast MAT 2D ${ }^{125} \mathrm{Te}$ NMR spectrum of $\mathrm{Ag}_{0.53} \mathrm{~Pb}_{18} \mathrm{Sb}_{1.2} \mathrm{Te}_{20}$ obtained with the pulse sequences of Figure 5.2 and sheared to provide isotropic shifts along $\omega_{1} "$ (vertical) and just center- and sidebands along $\omega_{2} "$ (horizontal). The centerband slice and the sum of first-order sideband slices are shown in Figure 5.3b and 5.3c, respectively. Peaks in the sideband slices are broader, most likely due to the greater sideband contribution from sites with larger chemical-shift anisotropies, which usually arise from a more disordered 
environment. The sum of all three slices is shown in Figure 5.3d (thick line) and compared with the regular 22-kHz MAS spectrum (thin line). The shoulders labeled by arrows in the regular 1D spectrum are unresolved spinning sidebands. These are seen to significantly increase the apparent signal of Te bonded to $\mathrm{Sb}$ around $-1500 \mathrm{ppm}$. As a result, the apparent $\mathrm{Te}\{\mathrm{Sb}\}$ fraction in the regular MAS spectrum is $30 \%$, while the actual number from MAT is $(22 \pm 3) \%$. This has important implications for clustering or segregation of $\mathrm{Sb}$ in this thermoelectric material. ${ }^{11,2}$ For instance, if all $\mathrm{Sb}$ was randomly placed on the $\mathrm{Pb}$ sublattice independent of $\mathrm{Ag}, 31 \%$ of all Te would have an $\mathrm{Sb}$ neighbor and resonate near $-1500 \mathrm{ppm}$; this is clearly not the case, indicating dopant clustering ${ }^{11}$ that may improve thermoelectric properties. ${ }^{4,11}$ The assignment of the "additional" signal in the 22-kHz MAS spectrum to sidebands is confirmed in Figure 5.3e, where the contributions from the Nth sideband obtained from MAT are shown (dotted lines) in their "natural" positions, $\omega_{\text {iso }}+\mathrm{N} \omega_{\mathrm{r}}$. In Figure 5.3e, the sum of these sidebands and the centerband (dashed line) is also compared with the regular MAS spectrum (continuous line). The good agreement shows that the additional pulses in MAT do not produce significant spectral distortions. The noise could be reduced further by time-domain filtering centered on the echo maximum.

$\mathrm{Sb}_{2} \mathrm{Te}_{3}$, also a good thermoelectric material, ${ }^{1}$ provides an example of a system with a wide spectrum, spanning $2 \pi 170 \mathrm{kHz}$, due to a large chemical-shift anisotropy reflecting its noncubic, rhombohedral structure ${ }^{12}$. The $2 \mathrm{D}$ fast MAT ${ }^{125} \mathrm{Te}$ NMR spectrum of $\mathrm{Sb}_{2} \mathrm{Te}_{3}$ is shown in Figure 5.4a, and Figure 5.4b compares the "infinite speed" MAS spectrum (thick line) obtained from the sum of the fast-MAT signal slices with the regular 22-kHz MAS spectrum (thin line). The sideband suppression greatly narrows the signal and exposes an asymmetry or shoulder to the left of the main peak, which could be due to the central Te layer in the layered Te-Sb-Te-Sb-Te crystal structure ${ }^{12}$. The comparison of the sum of MAT center- and sidebands in their "correct" positions with the regular MAS spectrum, see Figure 5.4c, confirms that fast MAT works well for large CSAs spanning $\sim 1.5 \gamma \mathrm{B}_{1}$.

The fast MAT spectra shown here and recorded on model compounds indicate that off-resonance spectral distortions are tolerable for a spectral range of $1.8 \gamma \mathrm{B}_{1}=200 \mathrm{kHz}=$ 1,600 ppm of ${ }^{125} \mathrm{Te}$ at $9.4-\mathrm{T}$ field. This range in ppm can be extended by (i) higher $\gamma \mathrm{B}_{1}$ values, which can be achieved in smaller radio-frequency coils ${ }^{13}$, or (ii) by measurements at 
lower $\mathrm{B}_{0}$ fields, where the spread of frequencies is reduced (fewer $\mathrm{Hz} / \mathrm{ppm}$ ). Both of these changes would be accompanied by signal reduction, so sensitivity limitations will impose the eventual estimated constraint of the spectral range to $\sim 4,000 \mathrm{ppm}$ of ${ }^{125} \mathrm{Te}$ or $\sim 6,000 \mathrm{ppm}$ of ${ }^{207} \mathrm{~Pb}$.

\section{Acknowledgements}

This work was supported by the U.S. Department of Energy - Basic Energy Sciences, Contract No. DE-AC02-07CH11358.

\section{Figures}

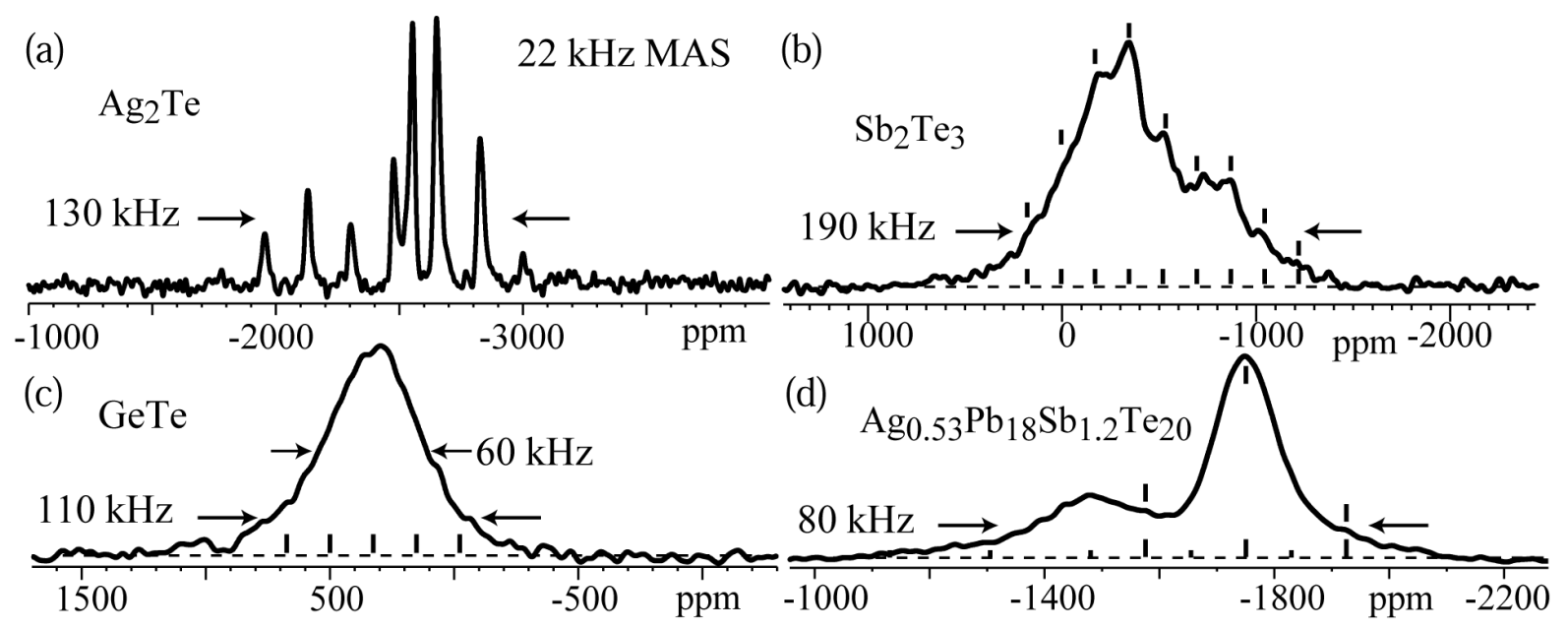

Figure 5.1. ${ }^{125} \mathrm{Te}$ NMR spectra of several tellurides, at 22-kHz MAS and 9.4 T. (a) $\mathrm{Ag}_{2} \mathrm{Te}$. (b) $\mathrm{Sb}_{2} \mathrm{Te}_{3}$. (c) $\mathrm{GeTe}$, a phase-change and thermoelectric material. (d) $\mathrm{Ag}_{0.53} \mathrm{~Pb}_{18} \mathrm{Sb}_{1.2} \mathrm{Te}_{20}$, a high-performance thermoelectric material. Spinning sideband positions are marked. The homogeneous linewidth $1 /\left(\pi \mathrm{T}_{2}\right)$ is $<7 \mathrm{ppm}$. 
(a) $\mathrm{t}_{\mathrm{ZA}}=\frac{\mathrm{t}_{\mathrm{r}}}{3}+\mathrm{Nt}_{\mathrm{r}}+\bmod \left(\mathrm{Mt}_{\mathrm{r}}-\frac{\mathrm{t}_{1}}{2}, \mathrm{t}_{\mathrm{r}}\right)-\mathrm{t}_{\mathrm{p}} \quad{ }_{\mathrm{t}_{\mathrm{p}}}^{\mathrm{p}_{\mathrm{r}}} \quad$ Refocus

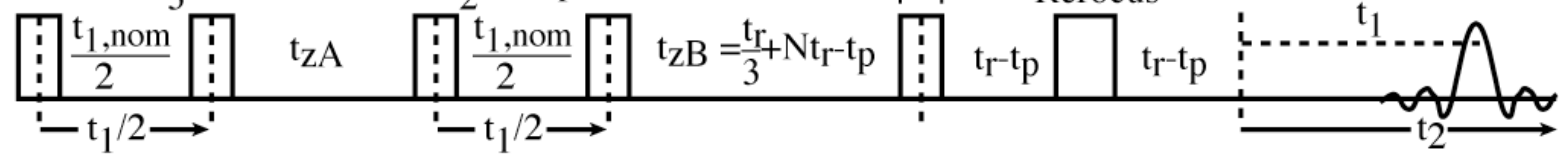

(b)

(c)

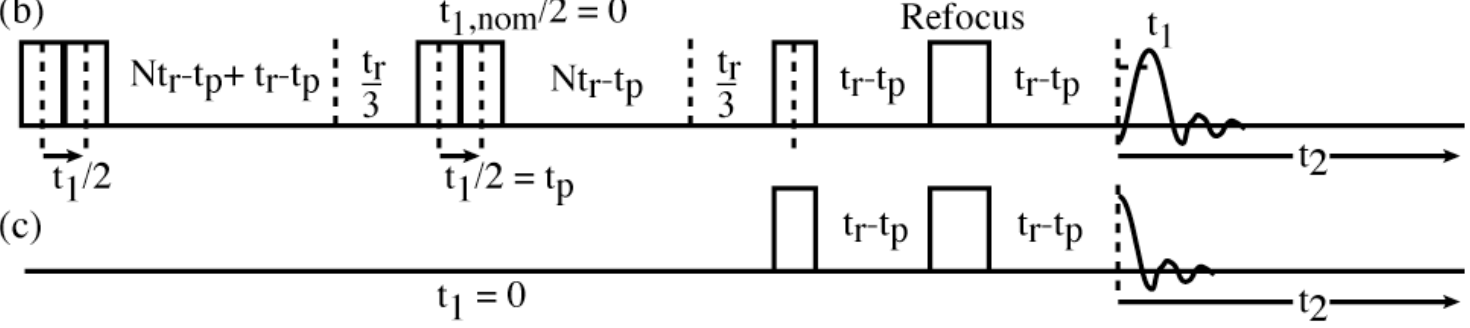

Figure 5.2. Fast magic-angle turning pulse sequence for broadband complete sideband suppression. (a) General pulse sequence, with two z-periods $t_{\mathrm{zA}}$ and $\mathrm{t}_{\mathrm{zB}}$. The unequal timings shown are for the " $t_{1}$ time reversed" $\mathrm{MAT}^{-}$data set ${ }^{9}$, while the $\mathrm{MAT}^{+}$data set is obtained with $t_{z B}=t_{z A}$, both decremented with increasing $t_{1} / 2$ [using a cyclic list from $t_{r}$ to $t_{p}$ (pulse duration) in steps of $-t_{p}$ ]. Flip angles can be $70^{\circ}$ to $90^{\circ}$ for the first five pulses, and $120^{\circ}-180^{\circ}$ for the last pulse. (b) Pulse sequence for nominal $t_{1, \text { nom }} / 2=0$, but effective $t_{1} / 2=t_{p}$. (c) Simplified pulse sequence for $\mathrm{t}_{1}=0$.

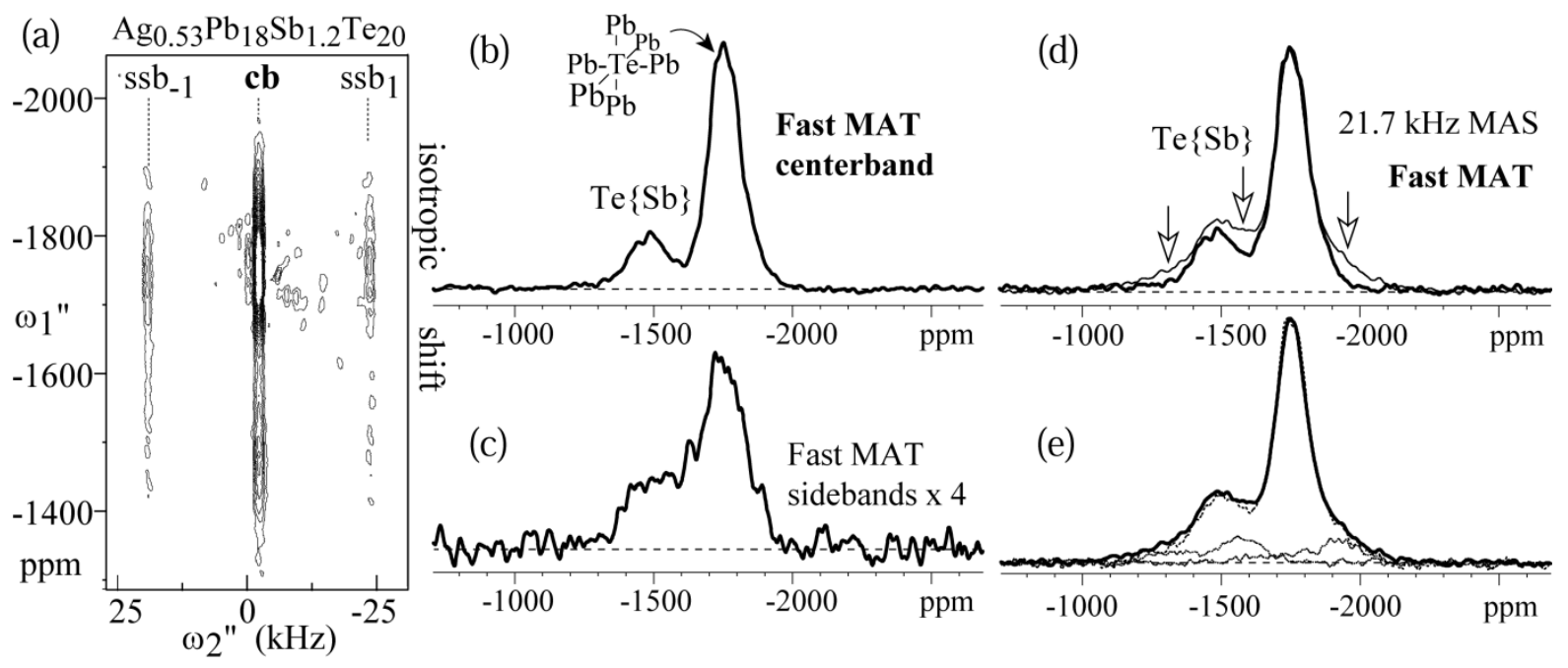

Figure 5.3. ${ }^{125} \mathrm{Te} \mathrm{NMR}$ spectra of $\mathrm{Ag}_{0.53} \mathrm{~Pb}_{18} \mathrm{Sb}_{1.2} \mathrm{Te}_{20}$. (a) $2 \mathrm{D}$ fast MAT spectrum after shearing along $\omega_{1}$ and $\omega_{2}$ providing the isotropic shift spectrum along $\omega_{1}$ " (vertical) and the 
sideband pattern along $\omega_{2} "$ (horizontal) Spinning frequency: $21.74 \mathrm{kHz} ; 80^{\circ}$-pulse length: 2 $\mu \mathrm{s}$; recycle delay: $150 \mathrm{~ms}$. (b) Centerband slice. (c) sum of sideband slices, scaled up fourfold. (d) Comparison of the "infinite-speed" spectrum from fast MAT (thick line, sum of center- and sideband slices) with the regular 21.74-kHz MAS spectrum (thin line). Arrows mark unresolved spinning sidebands in the regular spectrum. (e) Dotted lines (low-intensity bands): First-order sideband slices from MAT at their "natural" positions. Continuous line: regular 21.74-kHz MAS; dashed line: sum of MAT slices. Experiment time: $48 \mathrm{~h}$.

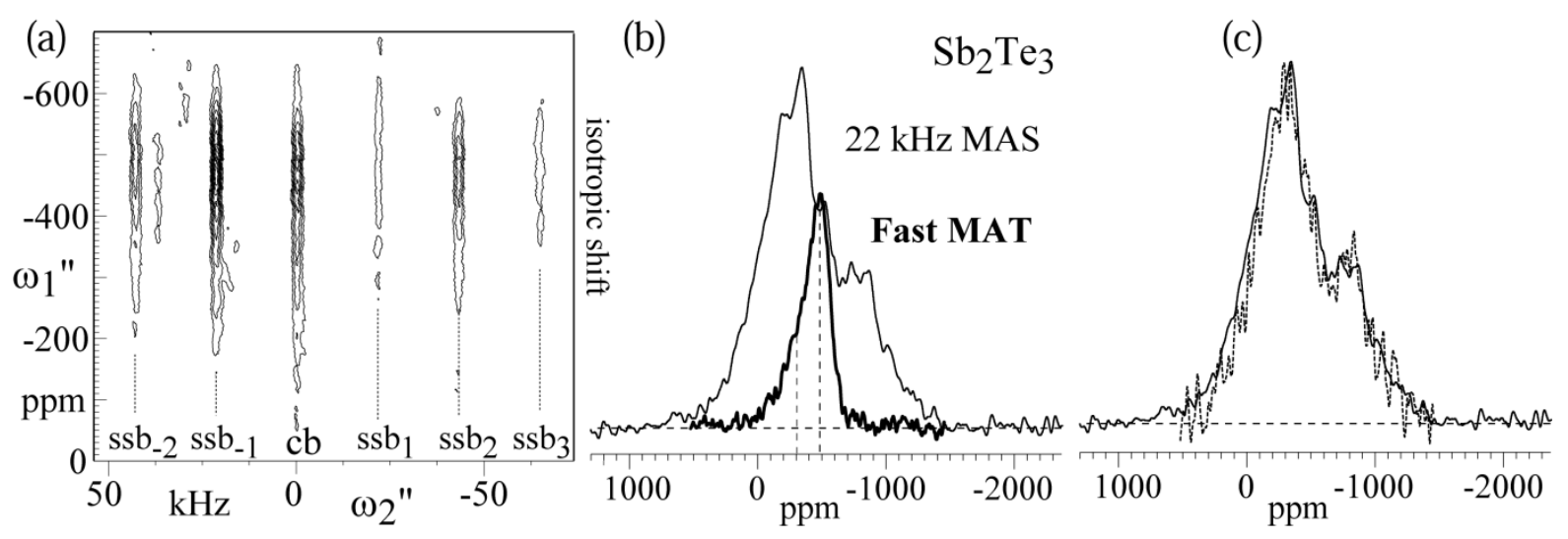

Figure 5.4. ${ }^{125} \mathrm{Te} \mathrm{NMR}$ spectra of $\mathrm{Sb}_{2} \mathrm{Te}_{3}$. (a) 2D fast MAT spectrum after shearing to provide the isotropic shift spectrum along $\omega_{1}$ " and the sideband pattern along $\omega_{2}$ ". Spinning frequency: $21.74 \mathrm{kHz}$; recycle delay: $50 \mathrm{~ms}$; experiment time: $36 \mathrm{~h}$. (b) Thick line: Isotropic chemical-shift spectrum from the sum of the signal slices in a). Thin line: regular $22-\mathrm{kHz}$ MAS spectrum for reference. (c) Comparison of regular 22-kHz MAS spectrum (continuous line) with the sum of MAT center- and sideband slices in their "natural" positions (dashed).

\section{References}

(1) Snyder, G. J.; Toberer, E. S. Nat. Mater. 2008, 7, 105-114.

(2) Hsu, K. F.; Loo, S.; Guo, F.; Chen, W.; Dyck, J. S.; Uher, C.; Hogan, T.; Polychroniadis, E. K.; Kanatzidis, M. G., Science 2004, 303, 818-821.

(3) Atwood, G. Science 2008, 321, 210-211. 
(4) Orion, I.; Rocha, J.; Jobic, S.; Abadie, V.; Brec, R.; Fernandez, C.; Amoureux, J.-P. J. Chem. Soc., Dalton Trans. 1997, 3741-3748.

(5) Dixon, W. T. J. Chem. Phys. 1982, 77, 1800-1809.

(6) Antzutkin, O. N.; Shekar, S. C.; Levitt, M. H. J. Magn. Reson., Ser. A 1995, 115, 719.

(7) Vogt, F. G.; Gibson, J. M.; Aurentz, D. J.; Mueller, K. T.; Benesi, A. J. J. Magn. Reson. 2000, 143, 153-160.

(8) Gan, Z. J. Am. Chem. Soc. 1992, 114, 8307-8309.

(9) Gan, Z. J.; Ernst, R. R. J. Magn. Reson. 1996, 123, 140-143.

(10) Garwood, M.; DelaBarre, L. J. Magn. Reson. 2001, 153, 155-177.

(11) Lin, H.; Bozin, E. S.; Billinge, S. J. L.; Quarez, E.; Kanatzidis, M. G. Phys. Rev. B:Condens. Matter 2005, 72, 174113/174111-174113/174117.

(12) Anderson, T. L.; Krause, H. B. Acta Cryst. 1974, 30, 1307-1310.

(13) Samoson, A. In Encyclopedia of NMR, 2002; Vol. 9, pp 59-64. Janssen, H.;Brinkmann, A.; Van Eck, E. R. H.; Van Bentum, P. J. M.; Kentgens, A. P. M. J. Am. Chem. Soc. 2006, 128, 8722-8723. Tang, J. A.; O'Dell, L. A.; Aguiar, P. M.; Lucier, B. E. G.; Sakellariou, D.; Schurko, R. W. Chem. Phys. Lett. 2008, 466, 227234. 


\title{
Chapter 6
}

\section{Technical Aspects of Fast Magic-Angle Turning NMR for Dilute Spin-1/2 Nuclei with Broad Spectra}

A paper submitted to Solid State Nuclear Magnetic Resoance

$$
\text { Y.-Y. Hu, K. Schmidt-Rohr }
$$

Ames Laboratory and Department of Chemistry, Iowa State University, Ames, Iowa 50011, USA

\begin{abstract}
For obtaining sideband-free spectra of high-Z $Z$ spin-1/2 nuclei with large (>1,000 ppm) chemical-shift anisotropies and broad isotropic-shift dispersion, we recently identified Gan's modified five-pulse magic-angle turning (MAT) experiment as the best available broadband pulse sequence, and adapted it to fast magic-angle spinning. Here, we discuss technical aspects such as pulse timings that compensate for off-resonance effects and are suitable for large CSAs over a range of $1.8 \gamma \mathrm{B}_{1}(1500 \mathrm{ppm})$; methods to minimize the duration of $\mathrm{z}$ periods by cyclic decrementation; shearing without digitization artifacts, by sharing between channels (points); and maximizing the sensitivity by echo-matched full-Gaussian filtering. The method is demonstrated on a model sample of mixed amino acids and its large bandwidth is heighted by comparison with a multiple- $\pi$-pulse technique. Applications to various tellurides are shown; these include GeTe, $\mathrm{Sb}_{2} \mathrm{Te}_{3}$ and $\mathrm{Ag}_{0.53} \mathrm{~Pb}_{18} \mathrm{Sb}_{1.2} \mathrm{Te}_{20}$, with spectra spanning up to $190 \mathrm{kHz}$, at $22 \mathrm{kHz}$ MAS. We have also determined the chemical shift anisotropies from the intensities of the spinning sidebands resolved by isotropic-shift separation.
\end{abstract}

\section{Introduction}

Magic-angle spinning NMR spectra of high-Z nuclei such as ${ }^{125} \mathrm{Te},{ }^{207} \mathrm{~Pb},{ }^{119} \mathrm{Sn}$, and ${ }^{113} \mathrm{Cd}$ in non-cubic environments are often crowded with spinning sidebands overlapped with the peaks at the isotropic shifts over a broad spectral range; this overlap interferes with the determination of isotropic chemical and Knight shifts, and evaluation of chemical shift anisotropies. Most methods designed for suppressing sidebands [1,2] employ long pulses 
and cannot achieve broadband excitation, leaving severely distorted spectra for spin systems of large spectral range. Recently, we adapted Gan's $\mathrm{MAT}^{ \pm}$experiment [3] to fast magic angle spinning with frequencies $>20 \mathrm{kHz}$, hereafter referred to as fast MAT [4], to maximize the sensitivity by solving pulse timing issues due to finite pulses and short rotation periods. Fast MAT is presently the best broadband method to separate isotropic chemical shift and chemical shift anisotropy. It has been successfully applied to various tellurides with spectral ranges up to $\sim 1.8 \gamma \mathrm{B}_{1}$ without significant spectral distortions [4].

In this paper, we detail technical aspects of the fast MAT experiment, including analysis and quantitative numerical simulations of the nearly linear off-resonance phase shift $=\mathrm{s}^{\cdot} \omega_{1} \mathrm{t}_{\mathrm{p}} / 2$, which can be considered as precession during the finite pulses and can therefore be included as an effective evolution time; and cyclic decrementation of the z-periods designed to keep them short in order to avoid $\mathrm{T}_{1}$ relaxation or possible spin diffusion without the loss of required synchronization. For data processing, we have employed echo-matched full Gaussian time-domain filtering to improve the signal-to-noise ratio, taking advantage of moving echoes (when $t_{2}=t_{1}$ ) due to the refocusing of the isotropic chemical shifts. We have also applied the sharing-between-channels approach [5] after shearing to avoid spectral artifacts associated with digital rounding when shearing parameters are not integers.

In addition, spectra acquired using PASS [2,6,7] and fast MAT under similar conditions on a model sample are used to compare these two methods in terms of sensitivity, spectral distortions, and experimental implementation. For spin systems with moderate spectral range, PASS is preferable due to its better sensitivity, and simpler experimental setup and data processing. However, when broad-range excitation is desirable, fast MAT demonstrates its reliability in producing sideband-free spectra without significant spectral distortions.

\section{Materials and Methods}

Samples. A mixture of seven amino acids with different ${ }^{13} \mathrm{C}$ labeling sites, including ${ }^{13} \mathrm{C}_{1^{-}}$ Leu, ${ }^{13} \mathrm{C}_{1}$-Gly, ${ }^{13} \mathrm{C}_{2}$-Leu, ${ }^{13} \mathrm{C}_{\alpha}$-Gly, ${ }^{13} \mathrm{C}_{3}$-Ala, ${ }^{13} \mathrm{C}_{1}$-Ala,${ }^{13} \mathrm{C}_{1}-\mathrm{N}_{\alpha}$-(tert-butoxycarbonyl)-Larginine, were used as a model sample. All of the labeled amino acids were purchased from 
CIL Inc. GeTe was used as received from GFS chemicals. Samples $\mathrm{Ag}_{0.53} \mathrm{~Pb}_{18} \mathrm{Sb}_{1.2} \mathrm{Te}_{20}$ and $\mathrm{Sb}_{2} \mathrm{Te}_{3}$ have been described in reference [8].

NMR parameters. All of the ${ }^{125} \mathrm{Te}$ NMR experiments were performed using a Bruker DSX400 spectrometer at $126 \mathrm{MHz}$ for ${ }^{125} \mathrm{Te}$. A $2.5-\mathrm{mm}$ MAS probe head was used at a spinning speed of $21.74 \mathrm{kHz}$ (synchronized with $\mathrm{t}_{1}$ increments) with ${ }^{125} \mathrm{Te} 90^{\circ}$ pulse length of $2.4 \mu$ s. All the fast MAT ${ }^{125} \mathrm{Te}$ spectra were obtained with the first five pulses of $2 \mu$ s and the last pulse of $3.5 \mu \mathrm{s}$ in order to achieve broad excitation. EXORCYCLE phase cycling [9] was used for pulse length error compensation for the last pulse. Recycle delays of $150 \mathrm{~ms}$ for $\mathrm{Ag}_{0.53} \mathrm{~Pb}_{18} \mathrm{Sb}_{1.2} \mathrm{Te}_{20}, 50 \mathrm{~ms}$ for $\mathrm{Sb}_{2} \mathrm{Te}_{3}$, and $50 \mathrm{~ms}$ for GeTe were applied. In the $\mathrm{S}^{-} \mathrm{MAT}$ datasets, the number of $\mathrm{t}_{1}$ increments was 400 for $\mathrm{Ag}_{0.53} \mathrm{~Pb}_{18} \mathrm{Sb}_{1.2} \mathrm{Te}_{20}, 280$ for $\mathrm{Sb}_{2} \mathrm{Te}_{3}$, and 320 for $\mathrm{GeTe}$, while in the $\mathrm{S}^{+}$MAT datasets, the number of $\mathrm{t}_{1}$ increments was reduced to 80

for $\mathrm{Ag}_{0.53} \mathrm{~Pb}_{18} \mathrm{Sb}_{1.2} \mathrm{Te}_{20}, 40$ for $\mathrm{Sb}_{2} \mathrm{Te}_{3}$, and 40 for GeTe. The ${ }^{125} \mathrm{Te}$ chemical shift relative to $\mathrm{Me}_{2} \mathrm{Te}$ in $\mathrm{CDCl}_{3}$ was calibrated using $\mathrm{TeO}_{2}$ at $+750 \mathrm{ppm}$ as a secondary reference.

For all ${ }^{13} \mathrm{C}$ NMR experiments on the model compounds, a 4-mm double resonance MAS probe head was used at a spinning speed of ca. $3 \mathrm{kHz}$. Weak ${ }^{13} \mathrm{C} 22-\mu \mathrm{s} 90^{\circ}$ r.f. pulses were purposely applied to test off-resonance tolerance of fast MAT and PASS. A reference spectrum using 5-pulse PASS was obtained with strong $4.4 \mu$ s $90^{\circ}$ r.f. pulses.

\section{Theoretical background and experimental setup}

Gan's MAT experiment. Gan's modified MAT experiment, $\mathrm{MAT}^{ \pm}$[3], includes two pulse sequences. One of them is identical to the original MAT [10] with $t_{\mathrm{zA}}=\mathrm{t}_{\mathrm{zB}}=\mathrm{t}_{\mathrm{r}} / 3-\mathrm{t}_{1} / 2$, generating the $\mathrm{MAT}^{+}$data set,

$$
S^{+}\left(t_{1}, t_{2}\right)=\left\langle\sum_{n=-\infty}^{\infty} S_{n} \exp \left\{i\left(\omega_{i s o}-n \omega_{r} / 2\right) t_{1}\right\} \cdot \exp \left\{i\left(\omega_{i s o}+n \omega_{r}\right) t_{2}\right\} \cdot R\right\rangle,
$$

where $t_{r}$ is the rotor period, $S_{n}$ is the intensity of the $n^{\text {th }}$ order spinning sideband, and $R$ accounts for relaxation effects. The other pulse sequence differs from the original one by an additional delay of $t_{1} / 2$ before the last read-out $90^{\circ}$ pulse, producing the "time-reversed" MAT $^{-}$data set, 
$S^{-}\left(t_{1}, t_{2}\right)=\left\langle\sum_{n=-\infty}^{\infty} S_{n} \exp \left\{-i\left(\omega_{i s o}-n \omega_{r} / 2\right) t_{1}\right\} \cdot \exp \left\{i\left(\omega_{\text {iso }}+n \omega_{r}\right) t_{2}\right\} \cdot R\right\rangle$.

The sum and difference of these two datasets create cosine and sine amplitude modulated 2D datasets, respectively, which can be Fourier transformed to yield purely absorptive 2D spectra. After spectral shearing, 2D spectra with an "infinite-speed" isotropic-shift projection along $\omega_{1}$ and anisotropic powder pattern or sidebands along $\omega_{2}$ are produced.

Gan's original MAT experiments were performed at slow spinning speeds for narrow spectral ranges $(<20 \mathrm{kHz})$, while for our purpose, spinning speeds greater than $20 \mathrm{kHz}$ are necessary to maximize the sensitivity and cover the spectral ranges larger than $100 \mathrm{kHz}$ needed due to wide chemical shift dispersion. Broadband spectra produce large phase shifts that can be explained as resulting from the evolution during pulses of finite duration. These lead to artifacts which can be corrected for as described below. Also, the rotor period $t_{r}$ becomes short at fast spinning and the $t_{1}$ evolution time has to exceed one $t_{r}$ to attain reasonable resolution, rather than $t_{1}<t_{r}$ at spinning speeds on the order of hundreds of hertz. Therefore, modifications of the durations of the z-periods ( $t_{\mathrm{ZA}}$ and $\mathrm{t}_{\mathrm{ZB}}$ ) are necessary to maintain pulse synchronization as well as avoid $\mathrm{T}_{1}$ relaxation and potential spin diffusion. Moreover, regular data processing procedures can be refined by employing echo-matched full Gaussian filtering to maximize the sensitivity and sharing between channels [5] after spectral shearing to eliminate spectral artifacts resulting from digital rounding with traditional shearing procedures.

Simulation of pulse excitation effects in fast MAT. In fast MAT experiments [4], four $90^{\circ}$ pulses are used in addition to the regular excitation and Hahn-echo pulse. Here, we analyze the effect of the pulses on magnetization off-resonance and show that the resulting phase shift, which is nearly linear with off-resonance frequency $\Delta \omega$, can be considered as precession during the pulses and thus compensated for.

The bandwidth of a single $90^{\circ} \mathrm{x}$-pulse applied to $\mathrm{z}$-magnetization is $2 \gamma \mathrm{B}_{1}$ if the full width at half maximum of the profile of the y-magnetization as a function of $\Delta \omega$ is considered [11]. However, the profile of the total transverse magnetization vs. $\Delta \omega$ is 
significantly wider, $>5 \gamma \mathrm{B}_{1}[12]$. The difference arises from a nearly linear off-resonance phase shift $\sim \Delta \omega \mathrm{t}_{\mathrm{p}} / 2[12]$ of the transverse magnetization.

The MAT sequence contains two pairs of $\sim 90^{\circ}$ pulses, with the first flipping zmagnetization to the transverse plane and the second restoring one magnetization component to $z$. It is instructive to start by considering one pair of ideal $\delta$-pulses. The transverse magnetization generated by the first $\mathrm{x}$-pulse precesses as

$$
\begin{aligned}
& \mathrm{M}_{\mathrm{y}}=\mathrm{M}_{0} \cos \Delta \omega \mathrm{t}_{1} ; \\
& \mathrm{M}_{\mathrm{x}}=\mathrm{M}_{0} \sin \Delta \omega \mathrm{t}_{1} .
\end{aligned}
$$

By the second pulse of suitable phase, either component can be stored along the z-direction:

$$
\begin{aligned}
& \mathrm{M}_{\mathrm{z}, \cos }=\mathrm{M}_{0} \cos \Delta \omega \mathrm{t}_{1} ; \\
& \mathrm{M}_{\mathrm{z}, \sin }=\mathrm{M}_{0} \sin \Delta \omega \mathrm{t}_{1} .
\end{aligned}
$$

The ratio of these two components can be written in terms of the evolution phase

$$
\begin{aligned}
& \phi=\Delta \omega \mathrm{t}_{1} ; \\
& \tan (\phi)=\mathrm{M}_{\mathrm{z}, \sin } / \mathrm{M}_{\mathrm{z}, \mathrm{cos}} .
\end{aligned}
$$

This relation will allow us to determine the effective duration of precession during pulses of finite length (Fig. 6.1a).

We have analyzed the excitation effect by numerically simulating the spin evolution during a back-to-back pulse pair (without any delay, nominal $t_{1, n o m}=0$ ) as a function of offresonance frequency $\Delta \omega$. The effective magnetic field in the rotating frame is calculated as $\overrightarrow{B_{e f f}}=\overrightarrow{B_{1}}+\overrightarrow{\Delta B_{0}}$, where $\Delta \omega=-\gamma\left|\overrightarrow{\Delta B_{0}}\right|$. The second pulse is phase cycled along $\pm \mathrm{y}$ and $\pm \mathrm{x}$ to obtain the cosine and sine components $\mathrm{M}_{\cos }$ and $\mathrm{M}_{\sin }$ of the magnetization, respectively. The \pm alternation of the pulse phase, combined with the corresponding alternation of the receiver phase, removes signal from residual transverse magnetization and distortions that arise for flip angles $\neq 90^{\circ}$. For each pulse, the precession of the magnetization around the effective magnetic field by an angle $\chi=\omega_{\text {eff }} \mathrm{t}_{\mathrm{p}}$ with $\omega_{\text {eff }}=-\gamma\left|\overrightarrow{B_{\text {eff }}}\right|$ is calculated using the rotation 
matrix $R_{\beta, \alpha}(\chi)=\tilde{R}(0,-\beta,-\alpha) \tilde{R}(\alpha, \beta,-\chi)[11]$, where $(\alpha, \beta)$ are the polar coordinates of the effective field ( $\alpha=0^{\circ}$ for an $\mathrm{x}-$, and $\alpha=90^{\circ}$ for a y-pulse, and $\beta=90^{\circ}$ for both) and $\tilde{R}$ is the standard active rotation matrix for the three Euler angles defining the rotation. The magnetization vector after the rotation is $\overrightarrow{M_{a f t e r}}=\mathrm{R}_{\beta, \alpha}(\chi) \overrightarrow{M_{\text {before }}}$. The initial magnetization is along the $\mathrm{z}$-axis, $\overrightarrow{M_{0}}=(0,0,1)^{\mathrm{T}}$. After pulse phase cycling as described above, the $\mathrm{z}$ magnetization components $\mathrm{M}_{\mathrm{z}, \mathrm{sin}}$ and $\mathrm{M}_{\mathrm{z}, \mathrm{cos}}$ are calculated as a function of $\Delta \omega$, in a range of \pm $4 \gamma B_{1}$. The length of the effective transverse magnetization during the pulses $\left(M_{\text {transv }}\right)$ is obtained as

$$
M_{\text {transv }}=\sqrt{M_{z, \sin }^{2}+M_{z, \cos }^{2}}
$$

while the effective evolution phase $\phi_{0}$ during the pulses can be defined analogous to Eq.(6.3) above:

$$
\tan \left(\phi_{0}\right)=\mathrm{M}_{\mathrm{z}, \sin } / \mathrm{M}_{\mathrm{z}, \cos }
$$

Fig. 6.1b shows that pulses with smaller flip angles give broader excitation profile than long pulses, as expected. Therefore, NMR methods aiming at achieving broadband excitation shall employ short r.f. pulses; however, there is usually a trade-off in terms of reduced sensitivity. Fig. 6.1c displays a plot of $\phi_{0} / \phi_{\mathrm{P}}$, i.e. the effective evolution phase normalized by the nominal pulse flip angle $\phi_{\mathrm{P}}=\omega_{1} \mathrm{t}_{\mathrm{p}}$, vs. $\Delta \omega / \omega_{1}$. The phase shift $\phi_{0} / \phi_{\mathrm{P}}$ is seen to grow linearly with $\Delta \omega / \omega_{1}$, with a slope $\mathrm{s} \approx 1$ for small flip angles and $\mathrm{s}=1.26$ for $90^{\circ}$ pulses. Therefore, after a pair of consecutive $90^{\circ}$ pulses, the effective evolution phase is

$$
\phi_{0}=\mathrm{s}\left(\Delta \omega / \omega_{1}\right) \phi_{\mathrm{P}}=\Delta \omega \mathrm{s} \mathrm{t}_{\mathrm{p}} .
$$

For a finite nominal evolution time $\mathrm{t}_{1, \text { nom }} / 2$,

$$
\phi=\Delta \omega \mathrm{t}_{1, \text { eff }} / 2=\phi_{0}+\Delta \omega \mathrm{t}_{1, \text { nom }} / 2=\Delta \omega\left(\mathrm{t}_{1, \text { nom }} / 2+\mathrm{s} \mathrm{t}_{\mathrm{p}}\right),
$$

which means that the $t_{1, \text { nom }} / 2=0$ slice of MAT corresponds to an effective evolution time $t_{1, \text { eff }} / 2=s t_{p}$. This results in a large distortion of the spectrum for nominal $t_{1, \text { nom }} / 2=0$. It can be partially removed by linear phase correction, but that requires time-consuming determination of the large correction phase and leaves residual baseline artifacts. To avoid this problem, we choose the increment of $t_{1} / 2$ as $t_{1, d w}=s t_{p}$, so that the phase of Eq.(6.6) for nominal $\mathrm{t}_{1, \text { nom }} / 2=0$ equals effective $\mathrm{t}_{1, \mathrm{eff}} / 2=\mathrm{t}_{1, \mathrm{dw}}=\mathrm{s} \mathrm{t}_{\mathrm{p}}$ (“second $\mathrm{t}_{1}$-slice"). The signal for 
effective $t_{1, \text { eff }} / 2=0$ is then approximated by separately recording the signal without the two pairs of storage and read-out pulses. Processing of $t_{1, \text { eff }} / 2=0$ signal enables determination of the optimum pre-detection delay and phase correction parameters in $\omega_{2}$. Later, the $t_{1, \text { eff }} / 2=0$ slice is incorporated into both the $\mathrm{S}^{+}$and $\mathrm{S}^{-} 2 \mathrm{D}$ spectra as the first slice in $\omega_{1}$. If the intensity of this "first slice" is somewhat too high compared to the rest because of pulse flip angles deviating from $90^{\circ}$, characteristic ridges are seen in the $2 \mathrm{D}$ spectrum parallel to the $\omega_{1}$ axis. These simple, distinctive artifacts can be eliminated by reducing the amplitude of the first slice by $\left(1-\cos 2 \phi_{\mathrm{p}} / 2\right)^{2}$. In our experiments using four $90^{\circ}$ pulses, nearly ideal weighting factors of 0.9 to 1 gave good results.

Cyclic decrementation of z-periods. The time between the start of the two evolution periods must be $\mathrm{nt}_{\mathrm{r}}+2 \pi / 3$ in MAT. Given that the first part of this time is the evolution period $\mathrm{t}_{1} / 2$, the delay $t_{\mathrm{zA}}$ between the end of the first and beginning of the next evolution period must be $t_{\mathrm{zA}}=\mathrm{nt}_{\mathrm{r}}+2 \pi / 3-\mathrm{t}_{1} / 2$. The rotation period $\mathrm{t}_{\mathrm{r}}$ is short at fast spinning and $\mathrm{t}_{\mathrm{z}}$ has to remain nonnegative. Rather than starting with a fixed long $\mathrm{nt}_{\mathrm{r}}$, which could result in spin exchange, $\mathrm{T}_{1}$ relaxation or desynchronization of the rotation and the pulse sequence, we use a cyclic delay list to minimize the length of the z-periods while preserving the necessary synchronization. For this purpose, the inter-pulse delay ( $\mathrm{t}_{\mathrm{ZA}}$ ) is split into two parts $t_{z A}=\frac{t_{r}}{3}+N t_{r}+t_{\mathrm{var}}$ (Fig. 6.2). One delay is fixed and equal to $t_{r} / 3$ minus the time needed for the intervening pulses, plus a couple of rotation periods for relaxation of transverse magnetization. The other delay, $t_{v a r}$, is varied and taken from a list with $n=t_{r} / t_{1, d w}$ entries, where $t_{1, d w}$ is the $t_{1} d$ well time and $t_{r}$ is chosen as an integer multiple of $t_{1, d w}$. The value of $t_{v a r}$ starts out with a value of $t_{r}$ for $t_{1}=$ 0 and is decremented in steps of $t_{1, d w}$. After $t_{v a r}$ has reached $t_{1, d w}$ in the cyclic list, it jumps back to $t_{r}$ for $t_{1}=n t_{r}$. For example, with $t_{1, d w}=2 \mu$ s and $t_{r}=46 \mu s$, the list is $\{46 \mu \mathrm{s}, 44 \mu \mathrm{s}, 42$ $\mu \mathrm{s}, \ldots, 6 \mu \mathrm{s}, 4 \mu \mathrm{s}, 2 \mu \mathrm{s}\}$. Since the delays are short, active synchronization of the pulse timing with the rotor phase is not necessary. 
Echoes and optimized filtering. For our purposes, dead-time free detection using a Hahn echo in fast MAT is indispensable, given that the time constants $\mathrm{T}_{2}{ }^{*}$ of the FIDs are $\sim 3 \mu \mathrm{s}$, if the line widths are $\sim 100 \mathrm{kHz}$.

In $\mathrm{MAT}^{ \pm}$experiments with powder averaging, Eq. (6.1) can be rearranged and written as

$$
\begin{aligned}
& S^{+}\left(t_{1}, t_{2}\right)=\left\langle\sum_{n=-\infty}^{\infty} S_{n} \exp \left\{i \omega_{\text {iso }}\left(t_{1}+t_{2}\right)\right\} \cdot \exp \left\{i n \omega_{r}\left(-t_{1} / 2+t_{2}\right\} \cdot R\right\rangle\right. \\
& S^{-}\left(t_{1}, t_{2}\right)=\left\langle\sum_{n=-\infty}^{\infty} S_{n} \exp \left\{i \omega_{\text {iso }}\left(-t_{1}+t_{2}\right)\right\} \cdot \exp \left\{i n \omega_{r}\left(t_{1} / 2+t_{2}\right)\right\} \cdot R\right\rangle .
\end{aligned}
$$

In Gan's MAT ${ }^{ \pm}$experiments with a spinning speed of $\sim 100 \mathrm{~Hz}$, notable echoes are observed at $\mathrm{t}_{2}=\mathrm{t}_{1} / 2$ in the $\mathrm{S}^{+}$dataset due to refocusing of the CSA. Rotational echoes occur at longer $t_{2}$. In fast MAT experiments, the sideband intensities are reduced by the fast spinning and therefore no significant echoes from CSA refocusing are detected, for instance, in the fast MAT experiments on the mixed amino acids.

However, given a broad distribution of isotropic chemical shifts $\omega_{\text {iso }}$ (i.e., chemical shift dispersion), the signal forms pronounced echoes at $t_{2}=t_{1}$ in the $S^{-}$dataset due to refocusing of isotropic chemical shifts in fast MAT. The echo dominates the observable signal, producing the sharp ridges shown in Figs. 6.4 and 6.5(a). Outside of the echo region, $\mathrm{t}_{1}-4 \mathrm{~T}_{2} *<\mathrm{t}_{2}<\mathrm{t}_{1}+4 \mathrm{~T}_{2} *$, noise dominates the observed time-domain signal. Therefore, the $\mathrm{S} / \mathrm{N}$ ratio of the spectrum can be improved by moving the maximum of a Gaussian smoothing function along with the echo maximum, as in full-echo detection schemes [13]. The $\mathrm{S} / \mathrm{N}$ ratio has been improved by this matched filtering for all the telluride samples studied as shown in Figs. 6.5, 6.6, and 6.7.

In contrast, the $S^{+}$dataset contains only an anti-echo term, $\left\langle\exp \left(i \omega_{i s o}\left(t_{1}+t_{2}\right)\right)>\right.$. As a result, for $\mathrm{t}_{1}>2 \mathrm{~T}_{2} *$, the intensity is negligible $(<2 \%)$ and does not need to be recorded, which provides a significant reduction in measuring time.

Spectral shearing in 2D fast MAT. The 2D spectra obtained directly from fast MAT contain tilted straight ridges (Figs. 6.3a and 6.4a) since according to eq. (6.7), both 
dimensions contain a mixture of isotropic chemical shifts and CSA [10]. Therefore, shearing is necessary to achieve a spectrum with peaks only at the isotropic shifts in the $\omega_{1}$ dimension and sidebands in the $\omega_{2}$ dimension for convenient data analysis.

The shearing procedure is demonstrated on the fast MAT 2D spectra of the model sample of mixed amino acids (Fig. 6.3) and of $\mathrm{Sb}_{2} \mathrm{Te}_{3}$ (Fig. 6.4). The chemical shift along $\omega_{1}$ is scaled by a factor of $2 / 3$ before shearing. As seen in Fig. 6.3, after shearing along $\omega_{1}$ by a factor of $1 / 2$, the sidebands are removed from the $\omega_{1}$ dimension, leaving the projection along $\omega_{1}$ to consist of a spectrum with isotropic shifts $\left(\omega_{1}{ }^{\prime}\right)$ (Fig. 6.3b). Subsequent shearing along $\omega_{2}$ by $-2 / 3$ yields a spectrum with projections of isotropic chemical shifts along the $\omega_{1}$ ' dimension and sidebands along the $\omega_{2}$ ' dimension (Fig. 6.3c).

The actual shearing tilt factors used on our commercial spectrometer are functions of shearing factors and numbers of data points in the $\omega_{1}$ and $\omega_{2}$ dimensions. For spin systems with well separated isotropic chemical shifts and well-spaced sidebands, the correct tilt factors can be obtained by reasonable trials of aligning related sidebands horizontally along $\omega_{2}$ and isotropic peaks vertically along $\omega_{1}$. In many cases with tellurides, the isotropic peaks are continuously distributed and the sidebands are merged (Fig. 6.4), so tilt factors need to be calculated as outlined in the supporting information to ensure correct shearing. After shearing (Fig. 6.4c), the ${ }^{125} \mathrm{Te}$ signals in the fast MAT 2D spectrum of $\mathrm{Sb}_{2} \mathrm{Te}_{3}$ are concentrated in vertical ridges. The cross sections along $\omega_{2}$ provide sideband patterns, and the analysis of their intensities can provide information on the chemical shift anisotropy associated with the selected isotropic shift in $\omega_{1}$ '.

Sharing between channels after spectral shearing. The common shearing method on our commercial spectrometer is via shifting columns or rows of the $2 \mathrm{D}$ matrix by moints, where $\mathrm{m}=$ tiltfactor $*(\mathrm{~N} / 2-\mathrm{n})$, in which the variable $\mathrm{N}$ is the total number of columns or rows and $\mathrm{n}$ is the column or row number. In most cases, $\mathrm{m}$ is not an integer and default digital rounding will cause inaccurate shearing and discontinuous profiles ("steps") in both dimensions of the sheared spectra. This problem can be circumvented by application of a sharing-between-channels approach [5]: in off-line data processing, when the number $\mathrm{m}$ falls 
between two integer values, the intensity of the matrix element that needs to be shifted is distributed between two entries $n+f$ floor $(m)$ and $n+$ floor $(m)+1$ in a row or column, with weighting factors of (floor $(m)+1-m)$ and $(m-f l o o r(m))$, respectively, where floor $(m)$ is the nearest integer smaller than $\mathrm{m}$.

Sideband artifacts. Due to pulse imperfections, in particular finite pulse length, the fast MAT 2D spectra can exhibit sideband artifacts, mostly at $\pm \omega_{\mathrm{r}}$ from the expected sidebands, in both spectral dimensions. Fortunately, these "first-order" sideband artifacts do not produce signal in the slices containing the real center- and sideband peaks, due to the slope of $-1 / 3$ of the sideband line (see dotted lines in Fig. 6.3c). Only third-order sideband artifacts coincide with signal slices, and these higher-order sidebands are negligibly small even for long r.f. pulses.

Quality test. Additional shearing along $\omega_{1}, \omega_{1}{ }^{\prime}=\omega_{1}{ }^{\prime}+\omega_{2}{ }^{\prime}$, can place the sidebands along $\omega_{1}$ " at their "natural" positions $\omega_{\text {iso }}+\omega_{\text {aniso }}$ as in the MAS spectrum (Fig. 6.4d). This enables us to experimentally deconvolute the 1D MAS spectrum into centerband and sideband contributions. Further, the sum of all signal slices should be identical to the regular 1D MAS spectrum. Thus, comparison between the regular MAS spectrum and the sum spectrum from triply sheared fast MAT is useful for assessing the extent of spectral distortions.

Doubling of 2D fast MAT spectra in $\omega_{1}$. Since the isotropic shifts of interest in our research span a wide range, shearing in the $\omega_{1}$ dimension can result in aliasing of off-resonance spectral regions. Although the spectra can be reconstructed to correct for aliasing, a simpler way is to replicate the spectra in the $\omega_{1}$ dimension by inserting pairs of slices with zero intensity into the $t_{1}$ time data so that they alternate with pairs of measured data slices [14].

The data processing using echo-matched full-Gaussian multiplication and sharing between channels after shearing was performed using the Matlab (Math Works, Natick, Massachusetts) software. 


\section{Results and Discussion}

Fast MAT on tellurides. The application of fast MAT to various tellurides demonstrates its ability of separating centerbands at the isotropic shift positions from spinning sidebands over a broad spectral range (Figs. 6.4 and 6.5a). Echo-matched full Gaussian filtering provides an improved signal-to-noise ratio.

Thin lines in Fig. 6.5b show the projections of the centerband and sidebands of the 2D fast MAT spectra of GeTe, with normal Gaussian multiplication and spectral shearing, and additional three-point filtering to further reduce noise, while the thick lines represent the corresponding spectra with echo-matched full-Gaussian filtering and sharing between channels after shearing. The $\mathrm{S} / \mathrm{N}$ ratio of the latter is significantly better even without additional three-point noise filtering, providing better defined spectral lineshapes of centerband and sidebands. Comparison between the regular MAS spectrum and the isotropic spectrum from fast MAT of GeTe in Fig. 6.5c reveals that the broad line width is largely due to a continuous distribution of isotropic chemical or Knight shifts over a wide range. An analysis of the chemical shift anisotropy based on the ratios of centerband and sideband integrals was carried out using matNMR (version 3.9.59) [15]. The sideband manifold calculation was performed employing a gammaCOMPUTE-based algorithm combined with powder averaging. Coarse optimization was facilitated by the SIMPLEX algorithm followed by a gradient minimization to finalize the analysis. The results show that chemical sites of isotropic chemical shifts between $270-450 \mathrm{ppm}$ have a slightly smaller $\Delta \sigma_{\mathrm{CSA}}$ of $240 \pm 30 \mathrm{ppm}$ than the rest with a $\Delta \sigma_{\mathrm{CSA}}$ of $280 \pm 20 \mathrm{ppm}$. With $\Delta \sigma_{\mathrm{CSA}}<2 \omega_{\mathrm{r}}\left(350 \mathrm{ppm}\right.$ for $\left.{ }^{125} \mathrm{Te}\right)$, the magnitude of the first-sideband integral $(<0.1)$ is almost independent of the asymmetry parameter $\eta$, which can therefore not be determined relaibly [16]. The agreement of the sum of centerband and sidebands at their "natural" positions in the triply sheared fast MAT with the regular MAS spectrum proves that fast MAT does not produce significant spectral artifacts over a spectral range $>\gamma B_{1}$ (Fig. 6.5d).

Similarly, for $\mathrm{Ag}_{0.53} \mathrm{~Pb}_{18} \mathrm{Sb}_{1.2} \mathrm{Te}_{20}$, the MAT spectral quality is improved in terms of $\mathrm{S} / \mathrm{N}$ ratio by use of the echo-matched full-Gaussian window function, especially for small sidebands (Fig. 6.6a). The purely isotropic spectrum (Fig. 6.6b) reconstructed from centerband and sidebands with improved S/N ratio (Fig. 6.6a) permits more accurate 
quantification of the percentage of Te bonded to $\mathrm{Sb}$, resonating around $-1500 \mathrm{ppm}$, which is $22 \%$ of the total Te. The comparison of the "infinite-speed" isotropic spectrum from fast MAT with the regular MAS spectrum in Fig. 6.6b sheds light on the interference of sidebands with the true intensity of each isotropic peak. Sideband analysis reveals that Te bonded to six $\mathrm{Pb}$ in the bulk lattice has a $\Delta \sigma_{\mathrm{CSA}}$ of $350 \pm 20 \mathrm{ppm}$, while Te bonded to $\mathrm{Sb}$ has a somewhat larger $\Delta \sigma_{\mathrm{CSA}}$ of $439 \pm 15 \mathrm{ppm}$. Similarly as in GeTe, the $\Delta \sigma_{\mathrm{CSA}}$ of $\mathrm{Ag}_{0.53} \mathrm{~Pb}_{18} \mathrm{Sb}_{1.2} \mathrm{Te}_{20}$ is comparable to $\omega_{\mathrm{r}}$ and $\eta$ cannot be determined. The good agreement of the sum of fast MAT centerband and sidebands in their "natural" positions with regular MAS (Fig. 6.6c) demonstrates that fast MAT results in no observable artifacts here.

The most dramatic difference made by using fast MAT to separate sidebands from the centerbands over a broad range is illustrated in the case of $\mathrm{Sb}_{2} \mathrm{Te}_{3}$. The large CSA, which reflects its non-cubic, rhombohedral structure [17], renders the lineshape of the isotropic spectrum unrecognizable even with $22 \mathrm{kHz}$ MAS. The centerband and sideband patterns in Figure 6.7 from fast MAT after processing with echo-matched filtering and sharing between channels after shearing show significantly reduced noise. The sideband pattern suggests that at least three inequivalent Te sites exist: the main peak in the middle with a $\Delta \sigma_{\mathrm{CSA}}$ of $1226 \pm 30 \mathrm{ppm}$ and an asymmetry parameter $\eta=0.45 \pm 0.1$ and the shoulder on both sides with a $\Delta \sigma_{\mathrm{CSA}}$ of $1306 \pm 30 \mathrm{ppm}$ and an asymmetry parameter $\eta=0.56 \pm 0.1$ (Fig. 6.7b). Fast MAT works quite well for the spectral range spanning over $\sim 1.8 \gamma \mathrm{B}_{1}$ as shown in the comparison of the regular MAS spectrum with the sum of all centerbands and sidebands in their "natural" positions of fast MAT, see Fig. 6.7c.

Comparison of PASS and fast MAT. Phase adjusted spinning sidebands (PASS) is a useful method for generating spectra of sidebands of order $n$, and after shearing, spectra with resonances only at the isotropic chemical shifts can be reconstructed. Dixon's 4- $(\pi)$ pulse PASS [6] was designed for lower spinning speeds, while at fast spinning, a multiple rotor cycle 5- $(\pi)$ pulse PASS version [2,7] was implemented to avoid pulse overlap and keep the total time constant. We compare fast MAT with PASS under the same experimental conditions. In terms of minimizing pulse excitation effects, 4-pulse PASS is preferable to 5pulse PASS. Due to relatively fast spinning and resulting short $t_{r}$ in our experiments, the 
slight variation $\left(<0.3 \mathrm{t}_{\mathrm{r}}\right)$ in total duration of 4-pulse PASS produces only negligible artifacts, smaller than an additional $180^{\circ}$ pulse.

For moderate spectral ranges, PASS is usually preferable over fast MAT. It has a higher sensitivity and can be implemented with a small number of increments and simple data processing. The very recent development of MAT-PASS by Hung and Gan [18] using linear $t_{1}$ increments allows for even easier implementation of PASS. On the other hand, the fast MAT experiment has a significantly wider excitation range and produces fewer sideband artifacts. Fig. 6.8 shows the comparison of spectra of seven mixed amino acids obtained by employing different versions of PASS and fast MAT under similar conditions, except the one using 5-pulse PASS with strong pulse excitation $(2 \pi 56.8 \mathrm{kHz})$, which is used as a reference of the undistorted spectrum. With weak pulses of $\gamma \mathrm{B}_{1}=2 \pi 11.4 \mathrm{kHz}$ over a spectral range of ca. 1.8 $\gamma \mathrm{B}_{1}$, fast MAT only yields minor spectral distortion, while for 5-pulse PASS, the distortion is overwhelming, leaving little or no intensity of all peaks. Even with relatively stronger pulses at $\gamma \mathrm{B}_{1}=2 \pi 21.9 \mathrm{kHz}$, the artifacts in the 4-pulse PASS spectrum are intolerable. Thus, PASS can sometimes serve as an exploratory method, and if PASS appears to produce sideband artifacts or distortions of off-resonance signals, then fast MAT can be used to generate reliable and high-quality spectra.

\section{Conclusions}

We have discussed technical details of fast MAT [4], including: i) the excitation effect of the pulses on magnetization off-resonance has been analyzed numerically, and the results show a phase shift nearly linear with off-resonance frequency $\Delta \omega$ with a factor close to unity for a pair of $<60^{\circ}$ pulses and 1.26 for $90^{\circ}$ pulses; this phase shift can be considered as resulting from precession during the pulses and thus compensated for by generating a slice with $t_{1, \text { eff }}=0$ for $2 \mathrm{D}$ MAT; ii) the duration of $\mathrm{z}$-periods have been minimized by cyclic decrementation, resolving issues from short rotation periods; iii) the sensitivity was optimized by matching the Gaussian filtering function with the maximum of the moving echoes generated from the refocusing of isotropic chemical shifts; iv) artifacts caused by digital rounding after shearing have been removed by the sharing-between-channels approach. The ${ }^{125} \mathrm{Te}$ chemical shift anisotropy parameters of various tellurium sites in 
tellurides have been determined by analysis of the sideband patterns at various isotropic shifts. The comparison between fast MAT and PASS reveals that fast MAT is more favorable for broadband excitation, with tolerable spectral distortions for large spectra range spanning up to $\sim 1.8 \gamma \mathrm{B}_{1}$.

\section{Acknowledgements}

This work was supported by the U.S. Department of Energy, Office of Basic Energy Science, Division of Materials Sciences and Engineering. The research was performed at the Ames Laboratory. Ames Laboratory is operated for the U.S. Department of Energy by Iowa State University under Contract No. DE-AC02-07CH11358.

\section{References}

[1] Dixon, W.T., J. Chem. Soc., Dalton Trans. 77 (1982) 3741-3748.

[2] O.N. Antzutkin, S.C. Shekar, M.H. Levitt, J. Magn. Reson. Ser. A 115 (1995) 7-19.

[3] Z.H. Gan, R.R. Ernst, J. Magn. Reson. Ser. A 123 (1996) 140-143.

[4] Y.Y. Hu, E.M. Levin, K. Schmidt-Rohr, J. Am. Chem. Soc. 131 (2009) 8390-8391.

[5] K. Schmidt-Rohr, Q. Chen, Nat. Mater. 7 (2008) 75-83.

[6] W.T. Dixon, J. Chem. Phys. 77 (1982) 1800-1809.

[7] F.G. Vogt, J.M. Gibson, D.J. Aurentz, K.T. Mueller, A.J. Benesi, J. Magn. Reson. 143 (2000) 153-160.

[8] E.M. Levin, B.A. Cook, K. Ahn, M.G. Kanatzidis, K. Schmidt-Rohr, Phys. Rev. B 80 (2009) 115211/1-115211/ 6.

[9] G. Bodenhausen, R. Freeman, D.L.Turner, J. Magn. Reson. 27 (1977) 511-514.

[10] Z.H. Gan, J. Am. Chem. Soc. 114 (1992) 8307-8309.

[11] K. Schmidt-Rohr, H.W. Spiess, Multidimensional Solid-state NMR and Polymers, Academic Press, San Diego, 1994.

[12] M. Garwood, L. DelaBarre, J. Magn. Reson. 153 (2001) 155-177.

[13] P.J. Grandinetti, J.H. Baltisberger, A. Llor, Y.K. Lee, U. Werner, M.A. Eastman, A. Pines, J. Magn. Reson. Ser. A 103 (1993) 72-81.

[14] S.F. Liu, J.D. Mao, K. Schmidt-Rohr, J. Magn. Reson. 155 (2002) 15-28. 
[15] J.D. van Beek, J. Magn. Reson. 187 (2007) 19-26.

[16] J. Herzfeld, A.E.Berger, J. Chem. Phys. 73 (1980) 6021-6029

[17] J.L.F. Da Silva, A. Walsh, H. Lee, Phys. Rev. B 78 (2008) 224111/1-224111/10.

[18] I. Hung, Z.H.Gan, J. Magn. Reson.204 (2010) 150-154.

\section{Figures}

(a)

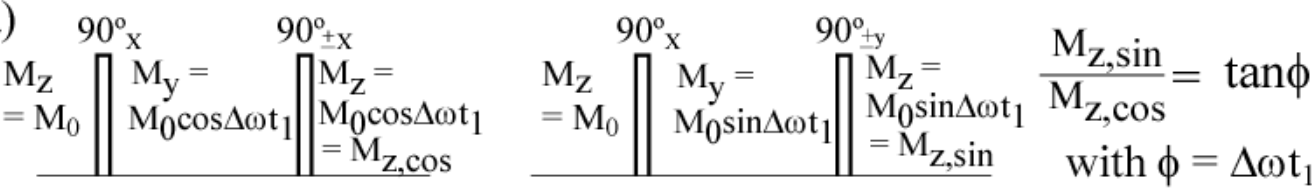

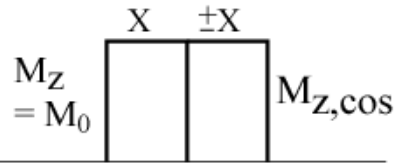

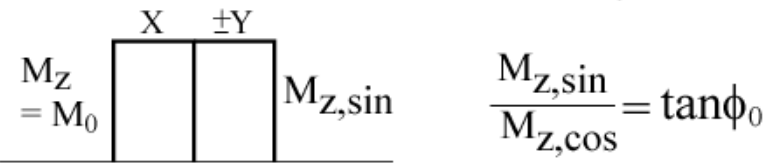
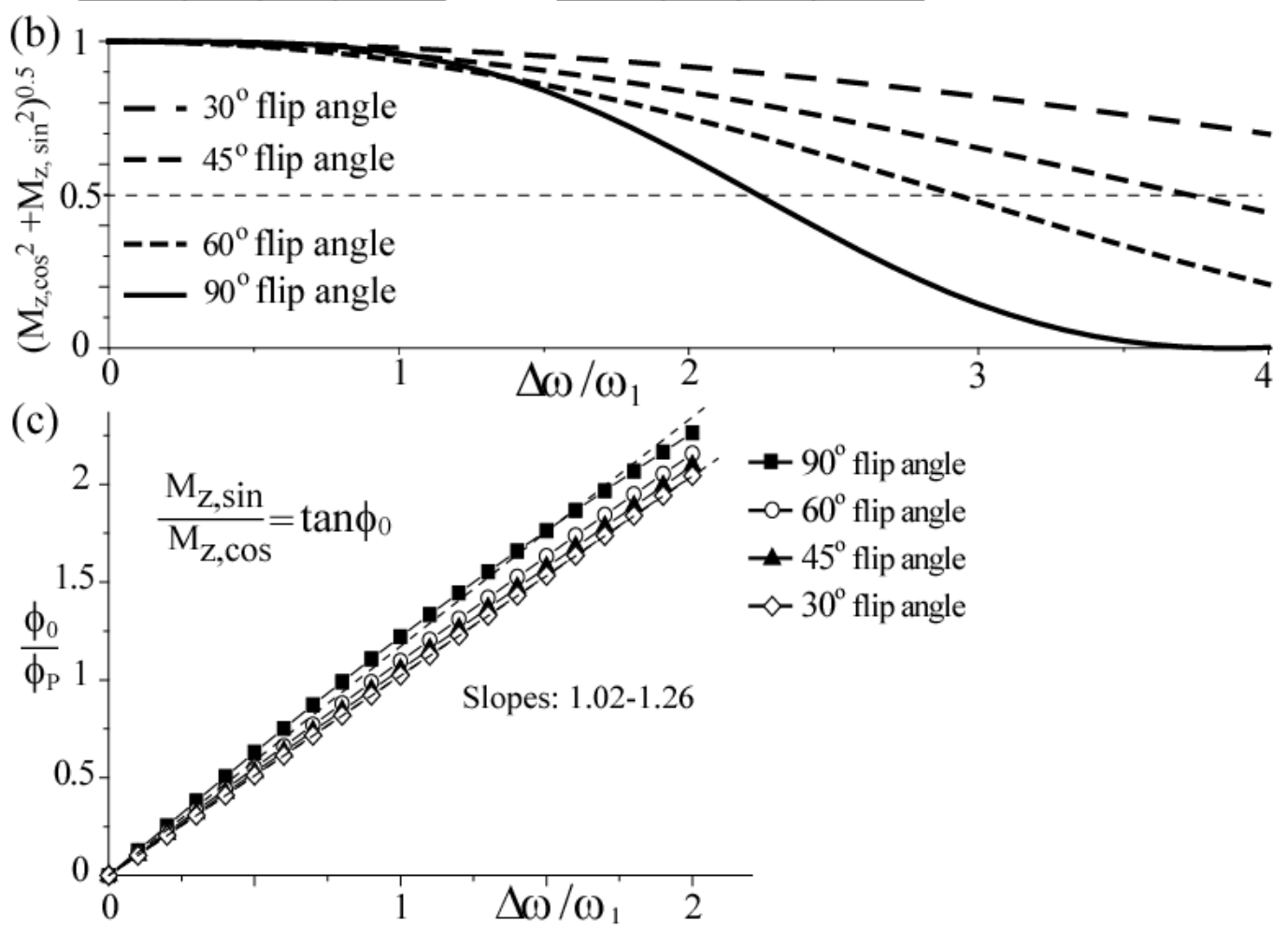

Fig. 6.1. Simulation of pulse excitation profiles and effects. (a) Pairs of $\delta$-pulses compared with pairs of back to back pulses of finite length. (b) "Magnitude" excitation profiles of a pair of back-to-back pulses of $30^{\circ}$ to $90^{\circ}$ flip angles. (c) Numerical simulation results of $\phi_{0} / \phi_{\mathrm{P}}$ 
with the nominal pulse flip angle $\left.\phi_{P}=\omega_{1} t_{p}\right)$ vs. $\Delta \omega / \omega_{1}$ for a pair of back-to-back pulses of $30^{\circ}$ to $90^{\circ}$ flip angles.

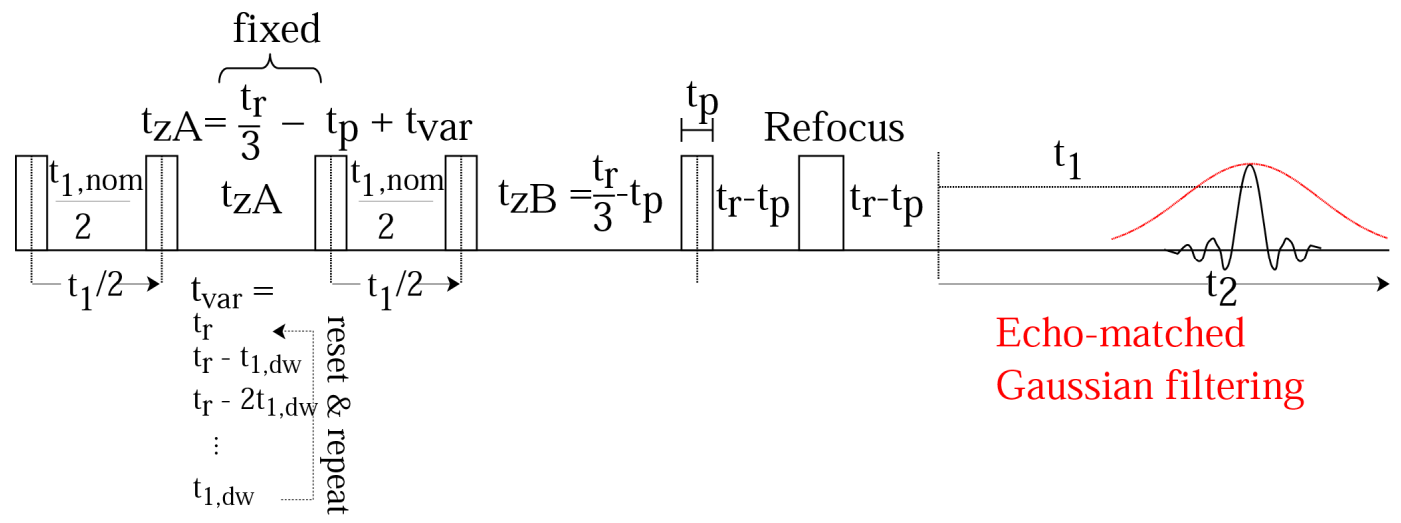

Fig. 6.2. Broadband fast MAT pulse sequence for obtaining pure isotropic-shift spectra and sideband separation. The unequal timings of the $\mathrm{z}$-periods shown are for the " $\mathrm{t}_{1}$ timereversed" $\mathrm{MAT}^{-}$dataset, while the $\mathrm{MAT}^{+}$data set is obtained with $\mathrm{t}_{\mathrm{zB}}=\mathrm{t}_{\mathrm{zA}}$, both decremented with increasing $t_{1} / 2$, illustrated by $t_{v a r}$ values taken from the delay list as shown. Flip angles can be $60^{\circ}-90^{\circ}$ for the first five pulses and $120^{\circ}-180^{\circ}$ for the last pulse. Echo-matched Gaussian filtering is applied to the time domain signal to optimize the sensitivity. 
(a)



(c)

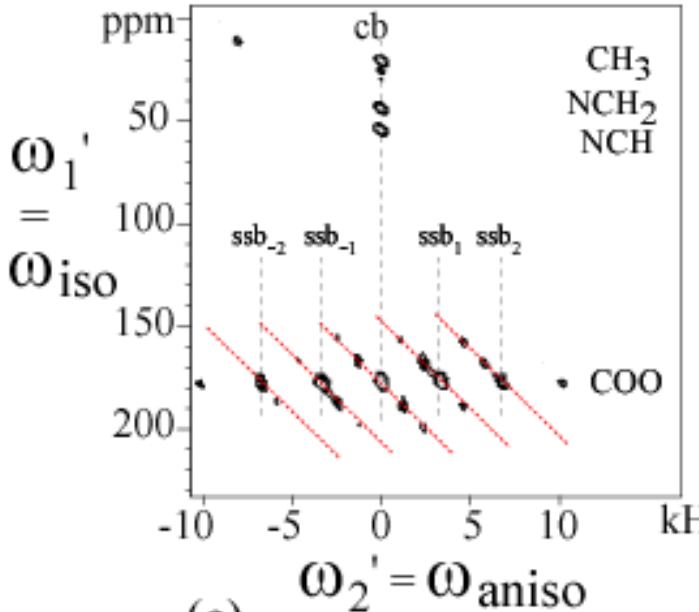

(e)

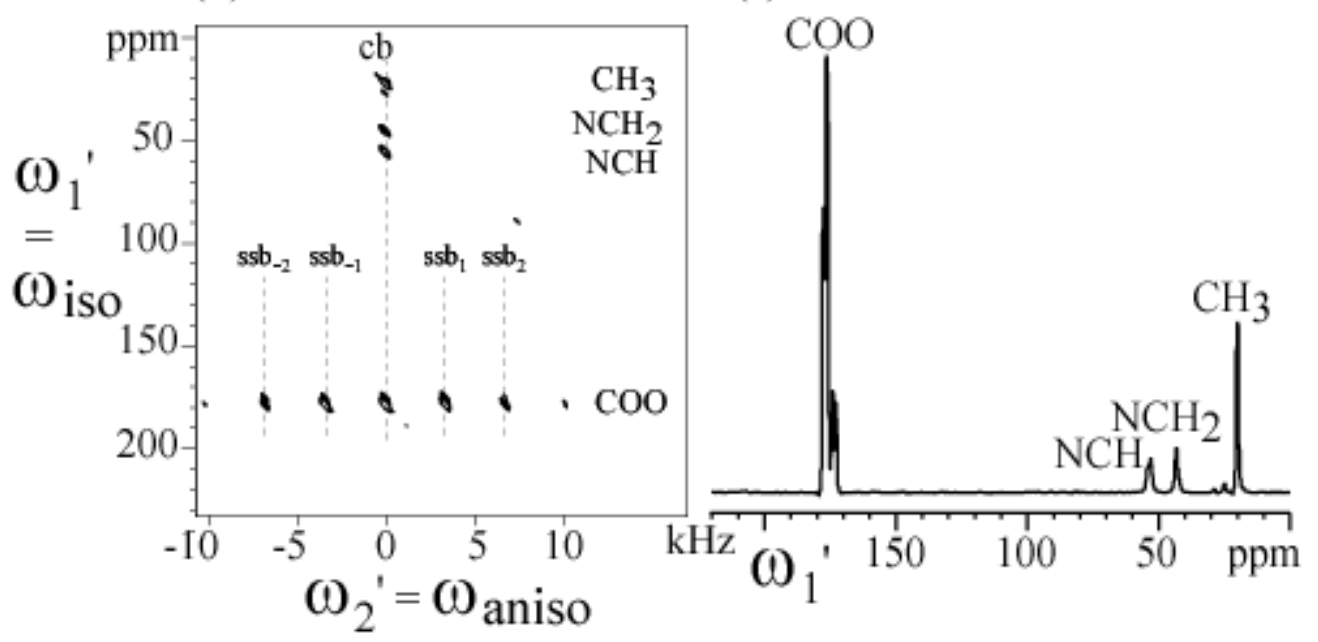

(b)

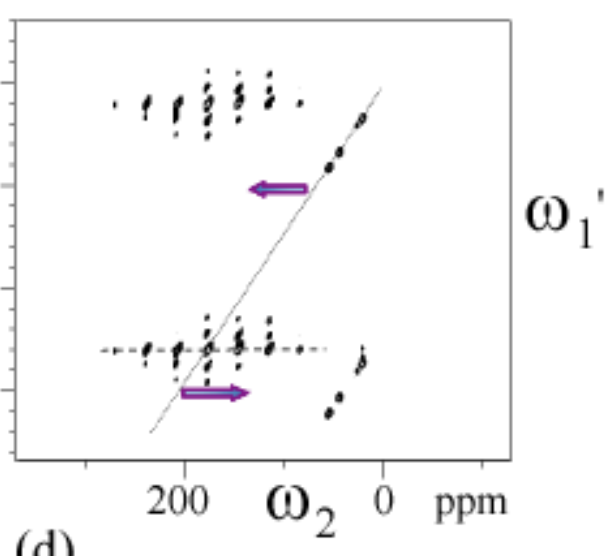

(d)

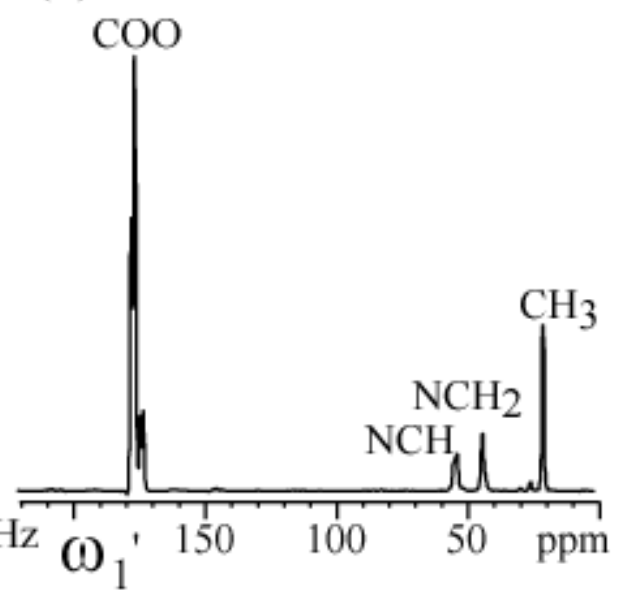

(f)

Fig. 6.3. Fast MAT spectra of a mixture of seven amino acids with different ${ }^{13} \mathrm{C}$ labeling $\left({ }^{13} \mathrm{C}_{1}\right.$-Leu, ${ }^{13} \mathrm{C}_{1}$-Gly, ${ }^{13} \mathrm{C}_{2}$-Leu, ${ }^{13} \mathrm{C}_{\alpha}$-Gly, ${ }^{13} \mathrm{C}_{3}$-Ala,,${ }^{13} \mathrm{C}_{1}$-Ala, ${ }^{13} \mathrm{C}_{1}-\mathrm{N}_{\alpha}$-(tert-butoxycarbonyl)-L- 
arginine) acquired with weak long pulses $\left(\gamma \mathrm{B}_{1} / 2 \pi=11.4 \mathrm{kHz}\right.$ ). (a) 2D fast MAT spectrum before shearing. The chemical shift was scaled by a factor of $2 / 3$ in the $\omega_{1}$ dimension. Each peak is replicated and shifted by $\omega_{\mathrm{r}}$ in $\omega_{1}$ by inserting zero-slices alternating with measured data in $t_{1}$. (b) After shearing along $\omega_{1}$. (c) After shearing along $\omega_{2}$. Sideband artifacts, due to long ( $22 \mu \mathrm{s})$ pluse length, are marked by slanted lines. (d) 1D spectrum of pure isotropic chemical shifts from the sum of centerband and sidebands generated from projections of the sheared fast MAT 2D spectrum in (c).

(a)

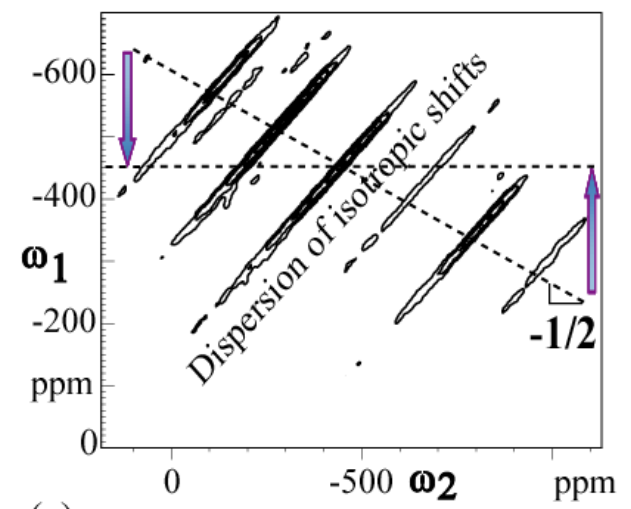

(c)

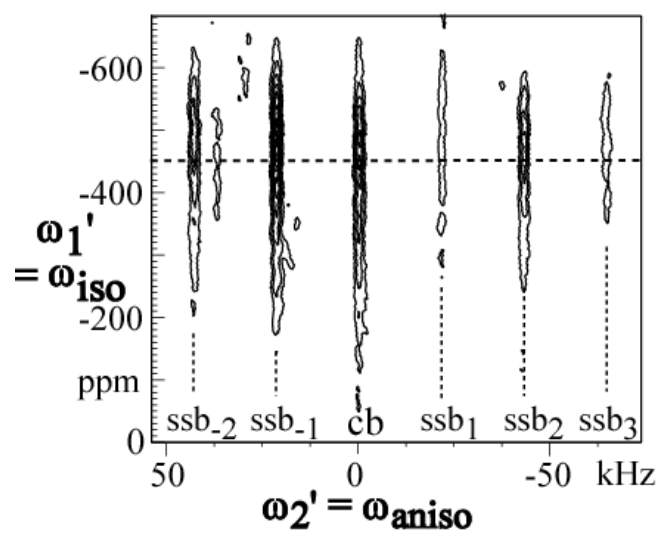

(b)

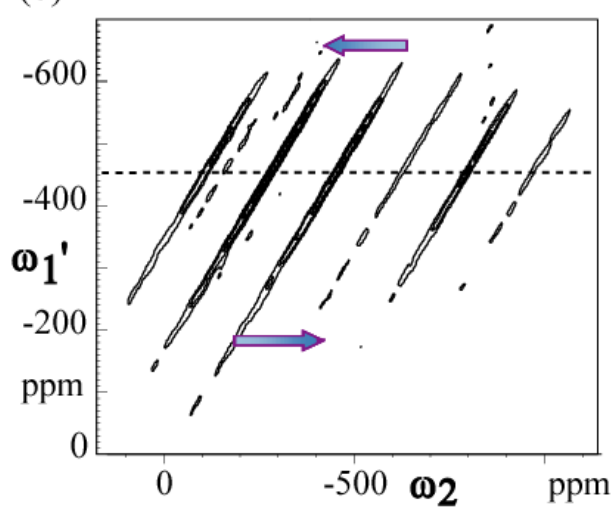

(d)

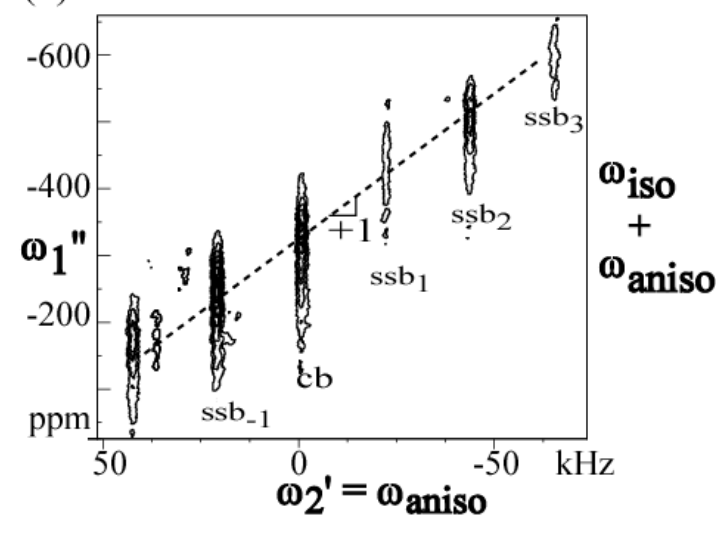

Fig. 6.4. Shearing of a fast MAT spectrum of $\mathrm{Sb}_{2} \mathrm{Te}_{3}$. (a) Original 2D fast MAT spectrum before shearing. The chemical shift was scaled by a factor of $2 / 3$ in the $\omega_{1}$ dimension. (b) After shearing along $\omega_{1}$ by $\arctan (-1 / 2)=-26.6^{\circ}$. (c) After further shearing along $\omega_{2}$ by $\arctan (-2 / 3)=-33^{\circ}$. Pure isotropic chemical shift along $\omega_{1}{ }^{\prime}$ and sidebands along $\omega_{2}{ }^{\prime}$ are obtained. (d) After further shearing along $\omega_{1}{ }^{\prime}$ by $\arctan (1)=45^{\circ}$ to achieve $\omega_{1}{ }^{\prime}=\omega_{1}{ }^{\prime}+\omega_{2}{ }^{\prime}=$ 
$\omega_{\text {iso }}+\omega_{\text {aniso }}$ for the purpose of a "quality test" and deconvolution of the regular MAS spectrum into subspectra of sideband order $n$.

(a)

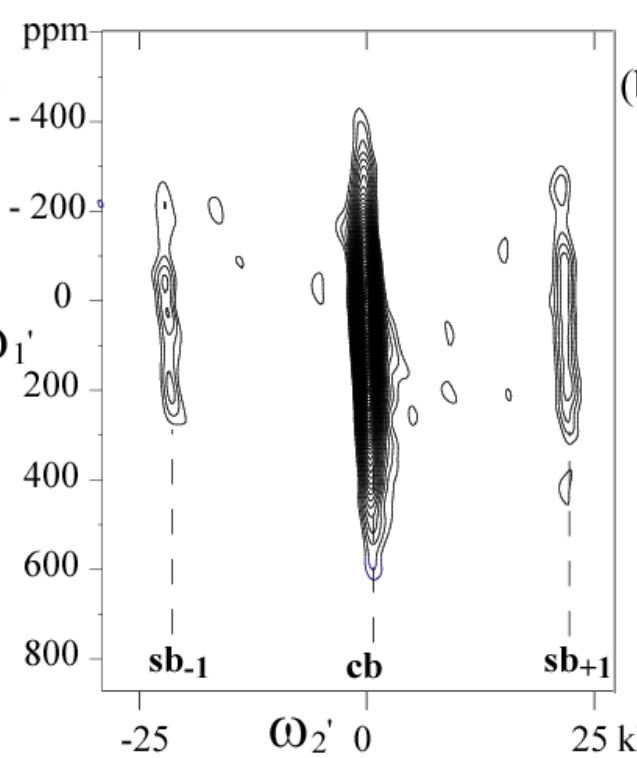

(c)

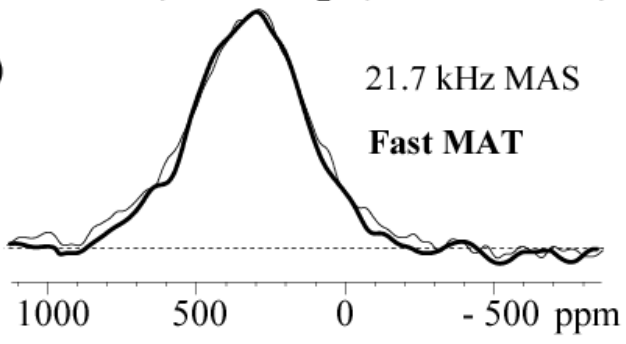

(b)
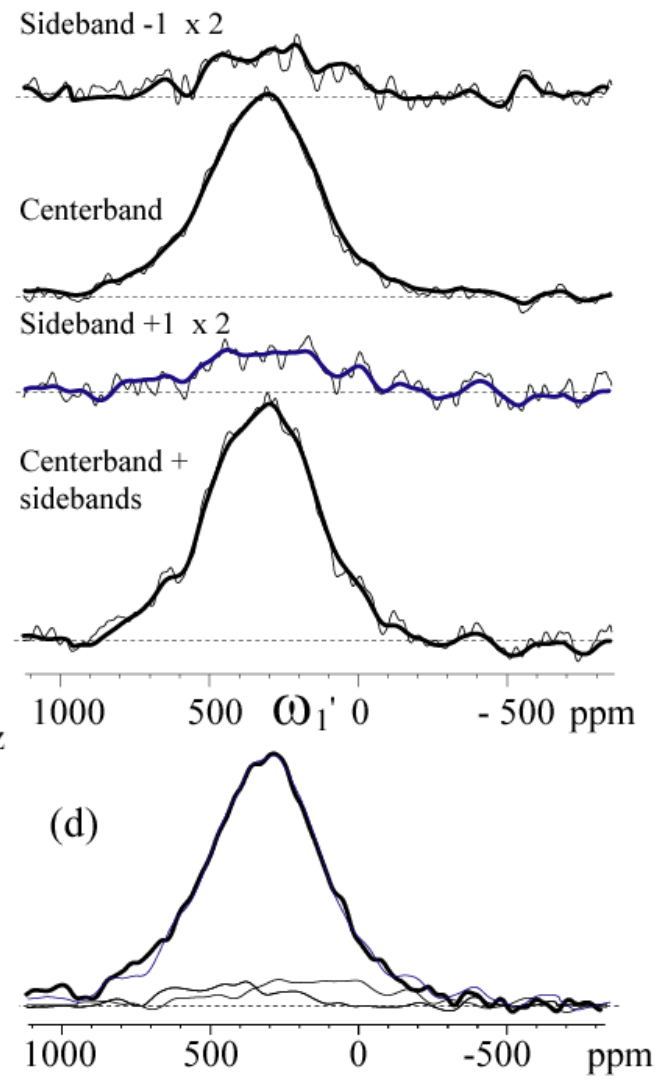

Fig. 6.5. ${ }^{125}$ Te NMR spectra of GeTe. (a) 2D fast MAT spectrum at $21.7 \mathrm{kHz}$ MAS with echo-matched full Gaussian multiplication and sharing between channels after shearing. (b) Projections of sidebands and centerband from the 2D fast MAT spectra with (thick lines) and without (thin lines) echo-matched full Gaussian multiplication and sharing-between-channels after shearing. (c) Regular $21.7 \mathrm{kHz}$ MAS spectrum (thin line) and isotropic spectrum of GeTe from fast MAT (sum of sidebands and centerband). (d) Quality test: comparison of regular $21.7 \mathrm{kHz}$ MAS spectrum (thick line) with the sum (thin line) of MAT center- and sideband slices in their "natural" positions; the latter are also shown individually (bands of low intensity). 
(a)

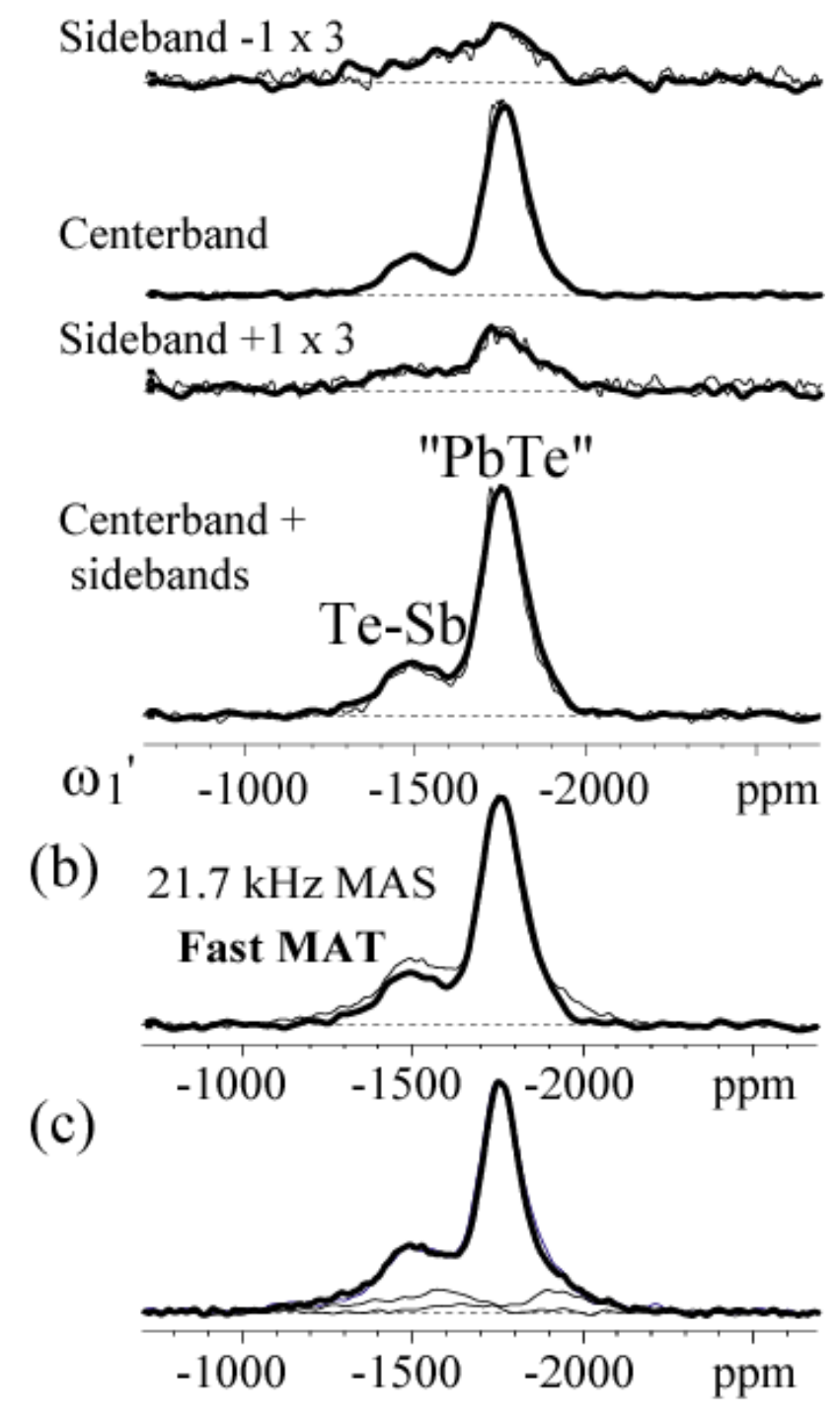

Fig. 6.6. ${ }^{125} \mathrm{Te} \mathrm{NMR}$ spectra of $\mathrm{Ag}_{0.53} \mathrm{~Pb}_{18} \mathrm{Sb}_{1.2} \mathrm{Te}_{20}$. (a) Projections of sidebands and centerband from the 2D fast MAT spectra with (thick lines) and without (thin lines) echomatched full Gaussian multiplication and sharing between channels after shearing. (b) Comparison of regular $21.7 \mathrm{kHz}$ MAS spectrum (thin line) and isotropic spectrum from fast MAT (sum of sidebands and centerband). (c) Quality test: comparison of regular $21.7 \mathrm{kHz}$ MAS spectrum (thick line) with the sum (thin line, barely distinguishable) of MAT centerand sideband slices in their "natural" positions; the latter are also shown individually (bands of low intensity). 
(a)
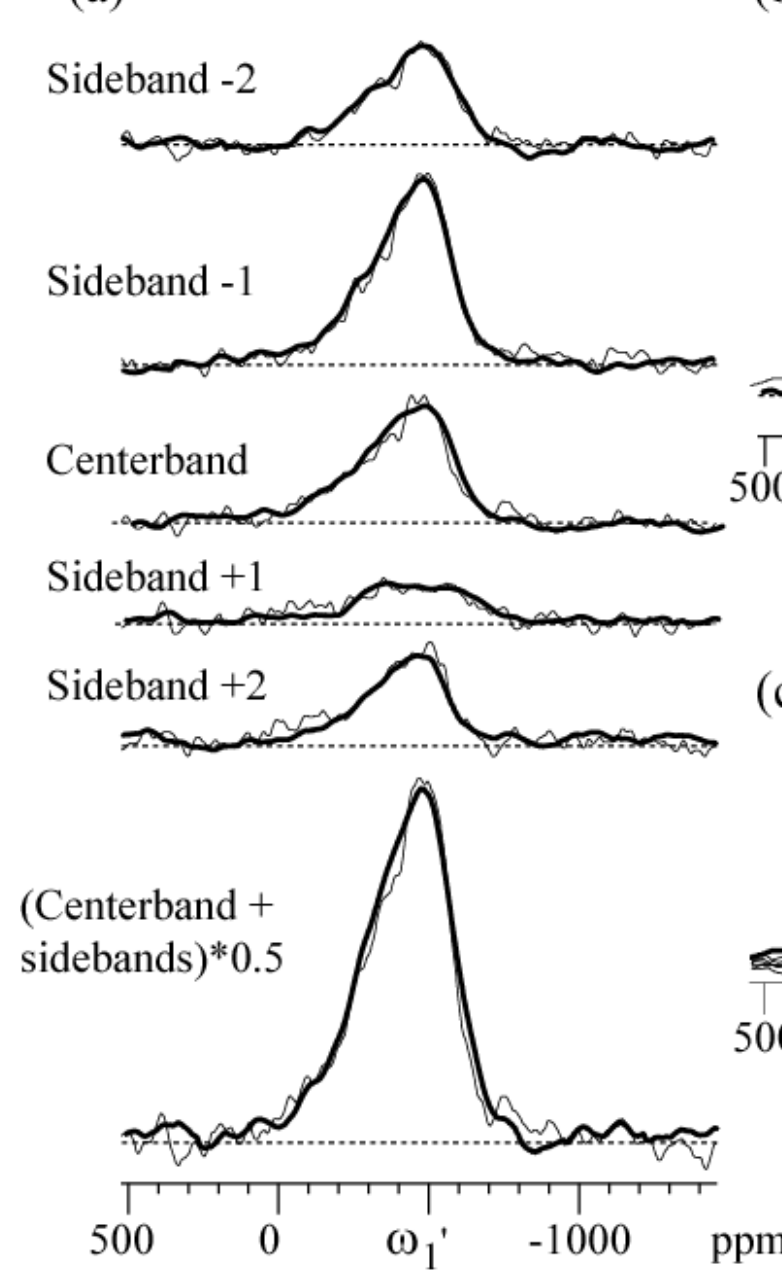

(b) $22 \mathrm{kHz}$ MAS
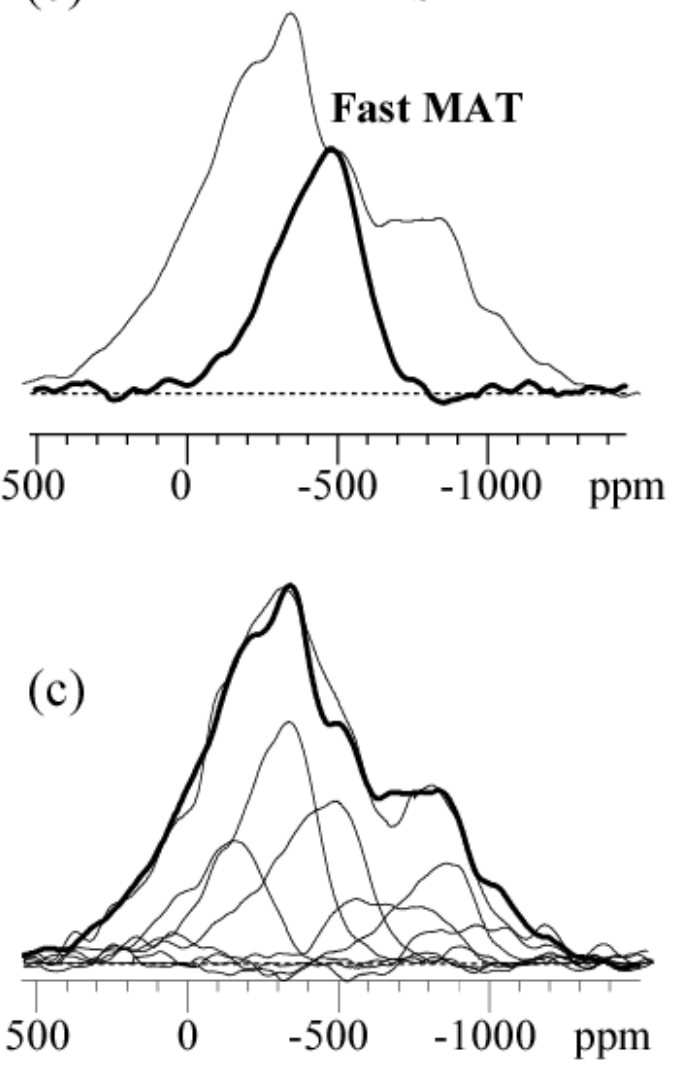

Fig. 6.7. ${ }^{125} \mathrm{Te} \mathrm{NMR}$ spectra of $\mathrm{Sb}_{2} \mathrm{Te}_{3}$. (a) Projections of sidebands and centerband from the 2D fast MAT spectra with (thick lines) and without (thin lines) echo-matched full Gaussian multiplication and sharing between channels after shearing. (b) Comparison of regular 21.7 $\mathrm{kHz}$ MAS spectrum (thin line) and isotropic spectrum from fast MAT (sum of sidebands and centerband). (c) Quality test: comparison of regular $21.7 \mathrm{kHz}$ MAS spectrum (thick line) with the sum (thin line) of MAT center- and sideband slices in their "natural" positions, which are also shown individually. 


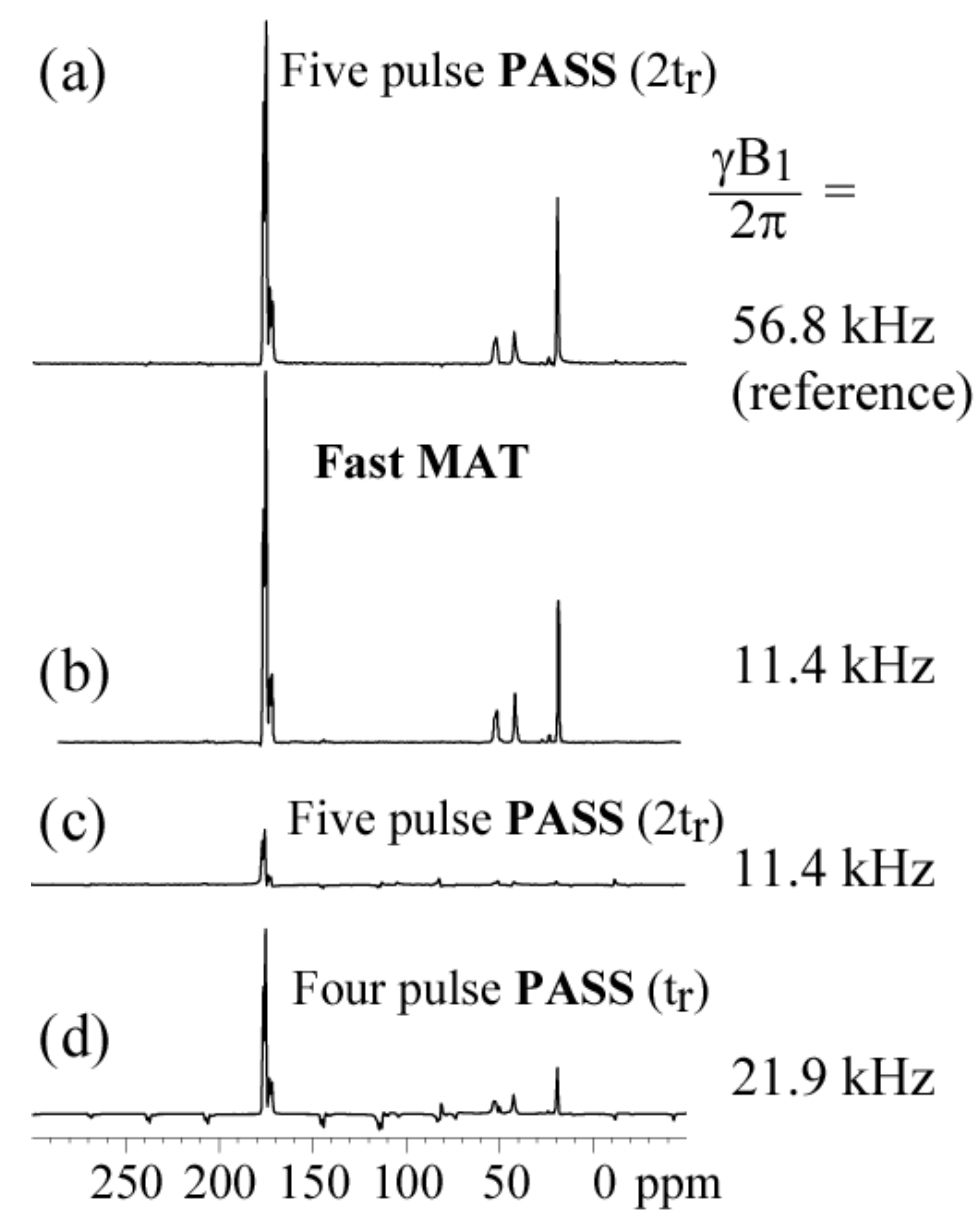

Fig. 6.8. Comparison of PASS and fast MAT demonstrated on a mixture of seven amino acids. 


\section{Supporting Information for}

\section{Technical Aspects of Fast Magic-Angle Turning NMR for Dilute Spin-1/2 Nuclei with Broad Spectra \\ Y.-Y. Hu, K. Schmidt-Rohr}

\section{Data acquisition and processing on a Bruker DSX spectrometer Data acquisition of fast MAT spectra}

(i) The $t_{1, \text { eff }}=0$ slice of a 2 DMAT spectrum is obtained by performing a 1D experiment using the pulse sequence in Fig. 6.2 but without the two pairs of readout and storage pulses.

(ii) Make a place holder for the $t_{1}=0$ slice in the $2 \mathrm{D}$ pulse program, and later incorporate the $1 D t_{1, \text { eff }}=0$ slice as the real $t_{1}=0$ slice into both the $\mathrm{S}^{+}$and $\mathrm{S}^{-}$ datasets (see data processing below).

(iii) During the acquisition of 2D datasets, insert a slice of zeroes in every other $\mathrm{t}_{1}$ slice in the time domain to replicate the 2D spectra in the $\omega_{1}$ dimension after Fourier transform

\section{Data processing of fast MAT spectra (with Xwin-NMR version 3.5)}

- Constructing the 2D absorptive spectra

(i) Incorporate the $1 \mathrm{D} t_{1, \text { eff }}=0$ spectrum into $\mathrm{S}^{+}$and $\mathrm{S}^{-}$using command "wser 1 <expno>" with the parameter Fcor in the edp window set as $0.5 * 0.25=0.125(0.5$ is for the regular first point scaling and 0.25 comes from the difference of phase cycles in the pulse programs for 1D and 2D data acquisitions), where <expno> is the experimental number of the $1 \mathrm{D}$ spectrum in the data file.

(ii) Fourier transform $\mathrm{S}^{+}$and $\mathrm{S}^{-}$over $\mathrm{t}_{1}$ and $\mathrm{t}_{2}$

(iii) For generating the cosine-dataset $S_{c}\left(\omega_{1}, \omega_{2}\right)$

$\mathrm{S}_{\mathrm{c}}\left(\omega_{1}, \omega_{2}\right)=\left(\mathrm{S}^{+}\left(\omega_{1}, \omega_{2}\right)+\mathrm{S}^{-}\left(\omega_{1}, \omega_{2}\right)\right) / 2(2$ positive diagonal ridges forming an $\mathrm{X})$, using add2d with $\alpha$ and $\gamma$ both set to 1 .

(iv) For generating the sine-dataset i $\mathrm{S}_{2}\left(\omega_{1}, \omega_{2}\right)=\left(\mathrm{S}^{-}\left(\omega_{1}, \omega_{2}\right)-\mathrm{S}^{+}\left(\omega_{1}, \omega_{2}\right)\right) / 2$ 
(a) Use wrpa to copy $S^{+}\left(\omega_{1}, \omega_{2}\right)$ and $S^{-}\left(\omega_{1}, \omega_{2}\right)$ to higher file numbers.

(b) In the edp window, add $90^{\circ}$ to the constant phase correction in both dimensions

of both copied spectra.

(c) Perform $\mathbf{x f b}$ on both datasets.

(d) Use wrpa to copy one of the datasets to a third file to subtract them using add2d with $\alpha=1$ and $\gamma=-1$.

iS $\mathrm{s}\left(\omega_{1}, \omega_{2}\right)$ has a positive and a negative diagonal ridge $\left(90^{\circ}\right.$ phase shift in $\left.\omega_{1}\right)$

(v) Produce $S_{\mathrm{c}}\left(\omega_{1}, \omega_{2}\right)+i S_{\mathrm{s}}\left(\omega_{1}, \omega_{2}\right)$ by using add2d with both $\alpha$ and $\gamma$ set to 1 .

(vi) Scale the chemical shift in $\omega_{1}$ by a factor of $2 / 3$.

- Spectral shearing

(i) Shear the $2 \mathrm{D}$ spectra first in $\omega_{1}$ dimension by setting " $1 \alpha "=-0.5 /(2 *$ (STSI of $\left.\omega_{1}\right) /\left(\right.$ STSI of $\left.\left.\omega_{2}\right)\right)$, and execute ptilt1.

(ii) Shear the $2 \mathrm{D}$ spectra in the $\omega_{2}$ dimension by setting $\alpha=(-2 / 3) /(($ STSI of $\left.\omega_{2}\right) /\left(2 *\left(\right.\right.$ STSI of $\left.\left.\omega_{1}\right)\right)$, and execute ptilt. 


\title{
Chapter 7
}

\section{Effects of L-spin Longitudinal Quadrupolar Relaxation in $\mathbf{S}\{\mathbf{L}\}$ Heteronuclear Recoupling and S-spin Magic-Angle Spinning NMR}

\author{
A paper published in Journal of Magnetic Resonance
}

2009, 197, 193-207

Y.-Y. Hu, K. Schmidt-Rohr

Ames Laboratory and Department of Chemistry, Iowa State University, Ames IA 50011

\begin{abstract}
In experiments on S-L heteronuclear spin systems with evolution of the S-spin magnetization under the influence of a quadrupolar nucleus (L-spin), effects of longitudinal quadrupolar $\left(\mathrm{T}_{1 \mathrm{Q}}\right)$ relaxation of the L-spin coherence on the sub-millisecond time scale have been documented and explored, and methods for minimizing their effect have been demonstrated. The longitudinal relaxation results in heteronuclear dephasing even in the reference signal $\mathrm{S}_{0}$ of $\mathrm{S}\{\mathrm{L}\}$ REDOR, REAPDOR, RIDER, or SPIDER experiments, due to $\mathrm{T}_{1 \mathrm{Q}}$-relaxation of the transiently generated $\mathrm{S}_{\mathrm{y}} \mathrm{L}_{\mathrm{z}}$ coherence, reducing or even eliminating the observable dephasing $\Delta \mathrm{S}$. Pulse sequences for measuring an improved reference signal $\mathrm{S}_{00}$ with minimal heteronuclear recoupling but the same number of pulses as for $S_{0}$ and $S$ have been demonstrated. From the observed intensity $\Delta \mathrm{S}_{0}=\mathrm{S}_{00}-\mathrm{S}_{0}$ and the SPIDER signal $\Delta \mathrm{S} / \mathrm{S}_{0}$, $\mathrm{T}_{1 \mathrm{Q}}$ can be estimated. Accelerated decays analogous to the dipolar $\mathrm{S}_{0}$ curves will occur in $\mathrm{T}_{2}$ measurements for J-coupled S-L spin pairs. Even in the absence of recoupling pulses, fast $\mathrm{T}_{1 \mathrm{Q}}$ relaxation of the unobserved nucleus shortens the transverse relaxation time $\mathrm{T}_{2 \mathrm{~S}}$, MAS of the observed nucleus, in particular at low spinning frequencies, due to unavoidable heteronuclear dipolar evolution during a rotation period. The observed spinning-frequency dependence of $\mathrm{T}_{2}$, MAS matches the theoretical prediction and may be used to estimate $\mathrm{T}_{1 \mathrm{Q}}$. The effects are demonstrated on several ${ }^{13} \mathrm{C}\left\{{ }^{14} \mathrm{~N}\right\}$ spin systems, including an arginine derivative, the natural $\mathrm{N}$-acetylated polysaccharide chitin, and a model peptide, (POG) 10 .
\end{abstract}




\section{Introduction}

Recoupling of heteronuclear dipolar interactions has proven extremely useful for structure determination[1,2]. While initially developed to probe the distance between two different isotopes with spin $1 / 2$, it has also been extended to quadrupolar nuclei. The REAPDOR [3,4] and SPIDER[5] methods use pulse sequences with most $180^{\circ}$ recoupling pulses applied to the observed spin-1/2 nucleus, see Fig. 7. 1, but differ in the pulses applied in the center of the recoupling sequence to invert or saturate the spin state of the quadrupolar nucleus. Recently, closely related methods for two-dimensional correlation of ${ }^{14} \mathrm{~N}$ and ${ }^{13} \mathrm{C}$ bands based on heteronuclear recoupling have also attracted significant attention[6,7].

In this paper, we discuss and demonstrate the effects of fast $\mathrm{T}_{1 \mathrm{Q}}$ relaxation of the quadrupolar nucleus on the $\mathrm{S}\{\mathrm{L}\}$ heteronuclear dephasing. Due to the large couplingstrength prefactors of the longitudinal relaxation rate, the $\mathrm{T}_{1 \mathrm{Q}}$ relaxation times of quadrupolar nuclei can be short, less than $0.1 \mathrm{~ms}$. In solution NMR, the very short $\mathrm{T}_{1 \mathrm{Q}}$ results in "selfdecoupling" of the L-spin from the S-spin[8,9,10]. In the solid state, it has usually been assumed implicitly that $\mathrm{T}_{1 \mathrm{Q}}$ exceeds the recoupling time $\mathrm{Nt}_{\mathrm{r}}$, but we show several experimental examples, namely a molecular crystal (an arginine derivative), a 30-residue collagen-mimetic peptide, and the biopolymer chitin, where this does not hold. We demonstrate a pulse sequence that has the same number and type of pulses as the standard $\mathrm{S}_{0}$ pulse sequence for recoupling involving heteronuclei with very broad spectra, but minimizes heteronuclear recoupling and thus the $\mathrm{T}_{1 \mathrm{Q}}$-relaxing two-spin coherence. The difference $\Delta \mathrm{S}_{0}$ between the resulting " $\mathrm{S}_{00}$ " signal and the standard $\mathrm{S}_{0}$ signal is dominated by peaks that fail to show the expected dephasing in SPIDER, as a result of reduced $\mathrm{S}_{0}$ intensity. $\mathrm{In}{ }^{14} \mathrm{~N}-{ }^{13} \mathrm{C}$ correlation spectra, such ${ }^{14} \mathrm{~N}$ sites with $\mathrm{T}_{1 \mathrm{Q}}$-relaxation on the 0.2 -ms time scale would not produce any signal. We derive the differential equations for simultaneous heteronuclear coupling and relaxation, with analytical solutions for $\mathrm{L}=1 / 2$ and numerical calculations for $\mathrm{L}=1 / 2,1$, and $3 / 2$, that produce the expected decrease of the apparent $\mathrm{T}_{2}$ of the observed $\mathrm{S}$ spin under MAS ( $\left.T_{2 S, M A S}\right)$ in $S_{0}$ measurements for $T_{1 Q} \approx 1 \mathrm{~ms}$, as well as the increase of $\mathrm{T}_{2 \mathrm{~S}}$, MAS and loss of heteronuclear evolution for very short $\mathrm{T}_{1 \mathrm{Q}}$, i.e. self-decoupling of the quadrupolar nucleus. 
Due to transient coupling effects during MAS, $\mathrm{T}_{1 \mathrm{Q}}$ relaxation of the quadrupolar heteronucleus also results in homogeneous line-broadening of the observed signal without any recoupling. The expected and observed increase in $T_{2 S}$, MAS with spinning frequency $\omega_{\mathrm{r}}$ matches well with the simulations, which provides an estimate of ${ }^{14} \mathrm{~N} \mathrm{~T}_{1}$. Finally, we also explore the origin of the enhanced ${ }^{14} \mathrm{~N} \mathrm{~T}_{1}$ relaxation in the crystalline arginine derivative in terms of ${ }^{13} \mathrm{C} \mathrm{T}_{1}$ relaxation "hot spots" and potential motional narrowing of chemical-shift powder patterns measured by the SUPER technique.

Note that the heteronuclear relaxation effect explored here is unrelated to the inhomogeneous line broadening due to nonsecular quadrupolar coupling acting on the heteronuclear dipolar coupling, which occurs in the absence of any motion for all ${ }^{13} \mathrm{C}-{ }^{14} \mathrm{~N}$ spin pairs and is most pronounced at low $\mathrm{B}_{0}$ field strengths[11]. In contrast, the relaxation effect of interest here requires fluctuations in the electric field-gradient tensor at the quadrupolar nucleus due to dynamic processes.

\section{Theoretical Background and Simulations}

To analyze heteronuclear evolution in the presence of $\mathrm{T}_{1 \mathrm{Q}}$ relaxation, we first consider the general situation, and then simulate the specific cases of an $\mathrm{S}$ spin coupled to an $\mathrm{L}=1 / 2, \mathrm{~L}=1$, or $\mathrm{L}=3 / 2$ spin.

Relaxation of L-spin Pseudo-Populations. Using product operators for the S-spin and matrix representations for the L-spin terms, the general reduced density matrix can be written as

$$
\begin{aligned}
\rho(t)= & S_{x} \operatorname{diag}\left(p_{x, L}, p_{x,(L-1)}, \ldots, p_{x,-(L-1)}, p_{x,-L}\right) \\
& +S_{y} \operatorname{diag}\left(p_{y, L}, p_{y},(L-1), \ldots, p_{y,-(L-1)}, p_{y,-L}\right)
\end{aligned}
$$

with time-dependent pseudo-populations in the diagonal L-spin matrices consisting of $(2 \mathrm{~L}+1)$ columns and rows. Normally, the diagonal elements of the density matrix correspond to populations of the corresponding energy levels. However, in the present case, some of the diagonal elements are negative; therefore, we refer to them as pseudo-populations. 
The exchange between the (pseudo-)populations due to longitudinal relaxation is controlled by a relaxation exchange matrix $\underline{\mathrm{W}}$ (also denoted as $\underline{\mathrm{R}}$ or $\underline{\mathrm{K}}$ in the literature) $[12,13]$.

$$
\mathrm{dp}_{\mathrm{x}, \mathrm{m}} / \mathrm{dt}=\Sigma_{\mathrm{m}^{\prime}=-\mathrm{L}}^{\mathrm{L}} \mathrm{W}_{\mathrm{m}, \mathrm{m}^{\prime}} \mathrm{p}_{\mathrm{x}, \mathrm{m}},
$$

The $\underline{\mathrm{W}}$ matrices for the first three $\mathrm{L}$ values are $[12,13]$

$$
\begin{aligned}
& \underline{\mathrm{W}}=\left(\begin{array}{cc}
-r & r \\
r & -r
\end{array}\right) \quad(\mathrm{L}=1 / 2) \\
& \underline{\mathrm{W}}=\left(\begin{array}{ccc}
-r_{1}-r_{2} & r_{1} & r_{2} \\
r_{1} & -2 r_{1} & r_{1} \\
r_{2} & r_{1} & -r_{1}-r_{2}
\end{array}\right) \quad(\mathrm{L}=1) \\
& \underline{\mathrm{W}}=\left(\begin{array}{cccc}
-r_{1}-r_{2} & r_{1} & r_{2} & 0 \\
r_{1} & -2 r_{1}-r_{2} & r_{1} & r_{2} \\
r_{2} & r_{1} & -2 r_{1}-r_{2} & r_{1} \\
0 & r_{2} & r_{1} & -r_{1}-r_{2}
\end{array}\right) \quad(\mathrm{L}=3 / 2)
\end{aligned}
$$

In the $\underline{\mathrm{W}}$-matrix for $\mathrm{L}=3 / 2$, it has been taken into account that the triple-quantum (and higher) matrix elements of electric quadrupolar and magnetic dipolar relaxation vanish[14], $r_{3}=0$. The matrix elements relate to the single- and double-quantum relaxation time constants $\mathrm{T}_{1 \mathrm{Q}}{ }^{\mathrm{SQ}}$ and $\mathrm{T}_{1 \mathrm{Q}}{ }^{\mathrm{DQ}}$ of spin $\mathrm{L}$ and the spectral densities of the dynamics as follows:

$$
\begin{aligned}
& \mathrm{r}=1 /\left(2 \mathrm{~T}_{1 \mathrm{~L}}\right)=\frac{3}{10} \gamma_{L}^{2} \gamma_{S}^{2} h^{2} r_{L S}^{-6}\left[\frac{1}{3} J\left(\omega_{L}-\omega_{S}\right)+J\left(\omega_{S}\right)+J\left(\omega_{L}+\omega_{S}\right)\right] \\
& \mathrm{r}_{1}=1 /\left(3 \mathrm{~T}_{1 \mathrm{Q}}{ }^{\mathrm{SQ}}\right)=\frac{1}{8}\left(\frac{e q Q}{h}\right)^{2} J^{(1)}\left(\omega_{0}\right) \\
& \mathrm{r}_{2}=1 /\left(3 \mathrm{~T}_{1 \mathrm{Q}}{ }^{\mathrm{DQ}}\right)=\frac{1}{4}\left(\frac{e q Q}{h}\right)^{2} J^{(2)}\left(2 \omega_{0}\right)
\end{aligned}
$$


It has been shown that $r_{1}$ and $r_{2}$ usually do not differ greatly [15,16]. For instance, isotropic motion gives $r_{1}=2 r_{2}$ in the slow-motion and $r_{1}=0.5 r_{2}$ in the fast-motion regime [16]. In most of our simulations, we assume $r_{1}=r_{2}$, but we will also explore the effects of the two rates not being equal. We have also neglected the orientation-dependence of the relaxation rates and times. For $T_{1 Q}>t_{r}$, this effect is minor since much of the variation is averaged out by MAS.

Further, $\mathrm{r}_{1}$ and $\mathrm{r}_{2}$ or $\mathrm{T}_{1 \mathrm{Q}} \mathrm{SQ}$ and $\mathrm{T}_{1 \mathrm{Q}} \mathrm{DQ}$ should be related to the easily observable longitudinal relaxation times $\mathrm{T}_{1 \mathrm{~L}}$ of $\mathrm{z}$-magnetization and $\mathrm{T}_{1 \mathrm{Q}}$ of the spin-alignment state for $\mathrm{L}$ $=1[16]$ :

$$
\begin{aligned}
& 1 / \mathrm{T}_{1 \mathrm{~L}}=\mathrm{r}_{1}+2 \mathrm{r}_{2} \\
& 1 / \mathrm{T}_{1 \mathrm{Q}}=3 \mathrm{r}_{1}
\end{aligned}
$$

When we assume $r_{1}=r_{2}$, all these equations simplify to $T_{1 L}=T_{1 Q}=T_{1 Q}{ }^{S Q}=T_{1 Q}{ }^{D Q}$. On this basis, we can refer to the quadrupolar $\mathrm{T}_{1 \mathrm{~L}}$ as $\mathrm{T}_{1 \mathrm{Q}}$ (which highlights the quadrupolar nature of the relaxation) without conflicting with the traditional definition.

Heteronuclear evolution in the presence of $\mathbf{T}_{1 Q}$ relaxation. We insert the general $\rho(t)$ of Eq.(7.1) into the von Neumann equation with relaxation

$$
\mathrm{d} \rho / \mathrm{dt}=-\mathrm{i} / \mathrm{h}\left[\mathrm{H}_{\mathrm{SL}}, \rho\right]+\underline{\mathrm{R}} \rho
$$

with the heteronuclear Hamiltonian

$$
\mathrm{H}_{\mathrm{SL}}=h \omega_{\mathrm{SL}} \mathrm{S}_{\mathrm{z}} \mathrm{L}_{\mathrm{z}}
$$

Under MAS and recoupling, $\omega_{S L}$ is the effective (average) frequency obtained after full rotation periods $\mathrm{Nt}_{\mathrm{r}}$. The action of the Redfield supermatrix $\underline{\mathrm{R}}$ on the $\mathrm{L}$-spin matrices is 
given by the effect of the regular matrix $\mathrm{W}$ on the L-spin population vectors, Eq.(7.2). As shown in Appendix A, inserting $\rho(t)$ of Eq. (7.1) into Eq. (7.6) gives coupled differential equations for the coefficients:

$$
\begin{aligned}
& \mathrm{dp}_{\mathrm{y}, \mathrm{m}} / \mathrm{dt}=\omega_{\mathrm{SL}} \mathrm{m} \mathrm{p}_{\mathrm{x}, \mathrm{m}}+\Sigma_{\mathrm{m}} \mathrm{W}_{\mathrm{m} \mathrm{m}^{\prime}} \mathrm{p}_{\mathrm{y}, \mathrm{m}}{ }^{\prime} \\
& \mathrm{dp}_{\mathrm{x}, \mathrm{m}} / \mathrm{dt}=-\omega_{\mathrm{SL}} \mathrm{m} \mathrm{p}_{\mathrm{y}, \mathrm{m}}+\Sigma_{\mathrm{m}}, \mathrm{W}_{\mathrm{m} \mathrm{m}} \mathrm{m}_{\mathrm{x}, \mathrm{m}},
\end{aligned}
$$

The initial condition for $\rho(0)=S_{x}$ magnetization is $p_{x, m}(0)=1$ and $p_{y, m}(0)=0$. The observable magnetization is obtained from the solution according to

$$
\left\langle\mathrm{S}_{\mathrm{X}}\right\rangle=\operatorname{tr}\left(\mathrm{S}_{\mathrm{x}} 1_{\mathrm{L}} \rho\right) \sim \Sigma_{\mathrm{m}} \mathrm{p}_{\mathrm{x}, \mathrm{m}}(\mathrm{t})
$$

For $\mathrm{L}=1 / 2$, two of the four equations are redundant and with $\mathrm{p}_{\mathrm{x},-1 / 2}=\mathrm{p}_{\mathrm{x},+1 / 2}=\mathrm{p}_{\mathrm{x}}$ and $\mathrm{p}_{\mathrm{y}, 1 / 2}=$ - $p_{y,+1 / 2}=-p_{y}$ we obtain (see Appendix B)

$$
\begin{aligned}
& \mathrm{dp}_{\mathrm{y}} / \mathrm{dt}=\mathrm{p}_{\mathrm{x}} \omega_{\mathrm{SL}} / 2-\mathrm{p}_{\mathrm{y}}\left(1 / \mathrm{T}_{2 \mathrm{~S}}+1 / \mathrm{T}_{1 \mathrm{~L}}\right) \\
& \mathrm{dp}_{\mathrm{x}} / \mathrm{dt}=\mathrm{p}_{\mathrm{y}}\left(-\omega_{\mathrm{SL}}\right) / 2-\mathrm{p}_{\mathrm{x}} / \mathrm{T}_{2 \mathrm{~S}}
\end{aligned}
$$

with transverse $T_{2 S}$ relaxation of the S-spin added in, as in the Bloch equations. The only difference from the standard Bloch equations is that the two-spin coherence $S_{y} L_{z}$ relaxes with the sum of the transverse relaxation rate of $S_{y}$ and the longitudinal relaxation rate of $L_{z}$. Similar "asymmetric relaxation" is also found in rotational resonance[17], spin exchange[18,19] and in cross polarization, with the same transition from oscillatory to exponential solutions.

Analytical solutions of Eq. (7.10) for the initial condition $\mathrm{p}_{\mathrm{x}}(0)=1, \mathrm{p}_{\mathrm{y}}(0)=0$ are given in Appendix B and plotted in Fig. 7. 2(a) for a series of $T_{1 L}$ values with $\omega_{S L}=2 \pi 1 \mathrm{kHz}$. For $\mathrm{T}_{1 \mathrm{~L}}>\mathrm{T}_{2 \mathrm{~S}}>1 / \omega_{\mathrm{SL}}$ the solution is the expected slightly damped $\cos \left(\omega_{\mathrm{SL}} / 2 \mathrm{t}\right)$ oscillation, but for $\mathrm{T}_{1 \mathrm{~L}}<1 / \omega_{\mathrm{SL}}$ it changes to a biexponential monotonous decay. For very short $\mathrm{T}_{1 \mathrm{~L}}$, a slow exponential decay is observed, 


$$
S(t)=\exp \left(-\omega_{S L}^{2} T_{1 L} t / 4\right)
$$

as shown in Appendix C. With the decay constant in Eqs.(11) and (C1b) inversely proportional to $T_{1 L}$, the decay becomes negligible for extremely short $T_{1 L}$, i.e. selfdecoupling is reproduced.

For $\mathrm{L}>1 / 2$, the analytical solutions are more complicated[8], and we have chosen instead to evaluate Eq. (7.8) numerically as outlined at the end of Appendix A. Sample results are plotted in Fig. 7. 3(a) for $\mathrm{L}=1$, and Fig. 7. $\mathrm{S} 1$ (a) for $\mathrm{L}=3 / 2$.

The consistency of the numerical simulations with the analytical simulations for $\mathrm{L}=$ $1 / 2$ confirms their accuracy. These simulations used a single frequency, which is relevant for dipolar coupling in single crystals and, more importantly, for a strong J-coupling to the quadrupolar nucleus (see discussion below). For long $\mathrm{T}_{1 \mathrm{Q}}$, we should recover the normal evolution

$$
\rho(\mathrm{t})=\mathrm{S}_{\mathrm{x}} \sum_{m=0 \text { or } 1 / 2}^{\mathrm{L}} \underline{1}_{\mathrm{m}} \cos \left(\mathrm{m} \omega_{\mathrm{SL}} \mathrm{t}\right)+\mathrm{S}_{\mathrm{y}} \mathrm{L}_{\mathrm{z}} \sum_{m=0 \text { or } 1 / 2}^{\mathrm{L}} \frac{1}{m} \underline{1}_{\mathrm{m}} \sin \left(\mathrm{m} \omega_{\mathrm{SL}} \mathrm{t}\right)
$$

where $\underline{m}_{\mathrm{m}}$ is a diagonal matrix with vanishing elements except for ones on the diagonal $\mathrm{m}$ position and $-\mathrm{m}$-position. For $\mathrm{m}=0$, the second term in Eq.(7.12) vanishes. Eq. (7.12) gives the expected signals

$$
\begin{array}{ll}
\mathrm{S}(\mathrm{t})=1 / 3+2 / 3 \cos \left(\omega_{\mathrm{SL}} \mathrm{t}\right) & \left(\mathrm{L}=1, \mathrm{~T}_{1 \mathrm{Q}}>2 \pi / \omega_{\mathrm{SL}}\right) \\
\mathrm{S}(\mathrm{t})=1 / 2 \cos \left(\omega_{\mathrm{SL}} \mathrm{t} / 2\right)+1 / 2 \cos \left(3 \omega_{\mathrm{SL}} \mathrm{t} / 2\right) & \left(\mathrm{L}=3 / 2, \mathrm{~T}_{1 \mathrm{Q}}>2 \pi / \omega_{\mathrm{SL}}\right)
\end{array}
$$

seen in Figs. 3(a) and $\mathrm{S} 1$ (a) for long $\mathrm{T}_{1 \mathrm{Q}}$. Simulated curves with powder averaging are shown in Fig. 7.2 (d), 3(d), and $\mathrm{S} 1(\mathrm{~d})$, for $\delta_{\mathrm{SL}}=2 \pi 1.5 \mathrm{kHz}$. For $\mathrm{L}=1 / 2$ and 1 at long $\mathrm{T}_{1 \mathrm{Q}}$, they reproduce the shape of the familiar REDOR curve. For $\mathrm{L}=1$, the long-time plateau is at an intensity of $1 / 3$ (see Eq. (7.13a)) due to the $m=0$ component with frequency 0 . At intermediate $T_{1 Q}$ values, the plateau slopes to $1 / 9$ because of relaxation of the $\mathrm{L}_{\mathrm{z}}{ }^{2}$ term of the $\mathrm{m}=0$ component in $\mathrm{S}_{\mathrm{x}}\left(1-\mathrm{L}_{\mathrm{z}}^{2}\right)[12,13]$. 
Refocusing of recoupling. In recoupling of heteronuclear interactions of an S-spin to an Lspin with a spectrum too broad for a train of inversion pulses, the recoupling pulses need to be applied to the $S$-spin; the reference signal $S_{0}$ is obtained by refocusing the recoupling effect, see Fig. 7. 1. In the center of the $S_{0}$ pulse sequence, at $t=\mathrm{Nt}_{\mathrm{r}} / 2$, a $180^{\circ}$ pulse is "missing", inverting the effective frequency. Thus, in the second half of the sequence, Eqs. $(8 \mathrm{a}, \mathrm{b})$ apply with $\omega_{\mathrm{SL}}$ replaced by $-\omega_{\mathrm{SL}}$. The intensity of the observable signal $\mathrm{S}_{0}\left(\mathrm{Nt}_{\mathrm{r}}, \mathrm{T}_{1 \mathrm{Q}}\right)$ for $L=1 / 2$ and different $T_{1 L}$ values, calculated in Appendix $B$, is shown in Fig. 7. 2(b) and coincides with the numerical results. Numerical simulations for $L=1$ and $L=3 / 2$ are plotted in Fig. 7. 3(b) and S1(b), respectively. Corresponding powder averaged $S_{0}$ curves are shown in Fig. 7. 2(e), 3(e), and S1(e), with $\delta_{\mathrm{SL}}=2 \pi 1.5 \mathrm{kHz}$.

Interestingly, noticeable oscillations with a frequency of $1 / 2 \mathrm{~m} \omega_{\mathrm{SL}}$ are observed in the $S_{0}$ curves at long to intermediate $T_{1 Q}$ values, in particular in Figs. 2(b) and 3(b). It appears that the magnetization at $\mathrm{Nt}_{\mathrm{r}}$ to some extent reflects the magnetization at $\mathrm{Nt}_{\mathrm{r}} / 2$, the time of the inversion of the effective frequency.

$\mathbf{T}_{1 \mathrm{Q}}$ effect on $\Delta \mathrm{S} . \Delta \mathrm{S}$ is obtained by subtracting $\mathrm{S}$ from $\mathrm{S}_{0}$, representing the dephasing due to heteronuclear recoupling. The simulated $\Delta \mathrm{S}$ curves for various $\mathrm{T}_{1 \mathrm{Q}}$ values are presented in Fig. 7. 2(c), 3(c), and S1(c) for a single frequency $\omega_{\mathrm{SL}}$ and in Fig. 7. 2(f), 3(f), and S1(f) for powder averaging with $\delta=2 \pi 1.5 \mathrm{kHz}$. These $\Delta \mathrm{S}$ curves for $\mathrm{L}=1 / 2,1$, and $3 / 2$ show similar patterns: In powder averaged simulations, for long $T_{1 Q}>100 / \delta$, the intensity of $\Delta S$ quickly rises to the maximum and then slightly oscillates around the plateau value, which is the ideal case for experiments like SPIDER, REDOR, and REAPDOR. For $\mathrm{T}_{1 \mathrm{Q}} \approx 100 / \delta$, the maximum is reduced by about $20 \%$ compared to the ideal value and the intensity decays fast after reaching the maximum. For $\mathrm{T}_{1 \mathrm{Q}}<10 / \delta$, the maximum intensity is reduced by more than $70 \%$. For $\mathrm{T}_{1 \mathrm{Q}}<<1 / \delta$, in the self-decoupling limit, no $\Delta \mathrm{S}$ signal can be obtained. In singlefrequency simulations, see Fig. 7. 2(c), 3(c), and S1(c), the amplitude of the oscillations is damped quickly as $\mathrm{T}_{1 \mathrm{Q}}$ decreases, until no intensity is observed when $\mathrm{T}_{1 \mathrm{Q}}$ is extremely short. 
$T_{1 Q}$ dependence of REDOR decay time constants in $S_{\mathbf{0}}$ and $S$. Decay time constants $T_{\mathrm{SL}}$ were extracted from the simulated single-frequency REDOR $\mathrm{S}$ and $\mathrm{S}_{0}$ curves for spin $\mathrm{L}=1$ shown in Fig. 7. 3(a),(b), and plotted as a function of $\mathrm{T}_{1 \mathrm{Q}}$ in Fig. 7. 4, for coupling strengths of $\omega_{\mathrm{SL}}=2 \pi 1 \mathrm{kHz}$ and $2 \pi 0.5 \mathrm{kHz}$. For our purpose, $\mathrm{T}_{\mathrm{SL}}$ was defined simply as the time of the initial decrease of the intensity to $\mathrm{e}^{-1}=0.37$. For small $\mathrm{T}_{1 \mathrm{Q}}<<1 / \omega_{\mathrm{SL}}, \mathrm{T}_{\mathrm{SL}}$ increases as $\mathrm{T}_{1 \mathrm{Q}}$ decreases, approaching more and more complete self-decoupling. In this range, the $\mathrm{S}$ and $\mathrm{S}_{0}$ curves coincide: The only difference between $S$ and $S_{0}$ in REDOR is the inversion pulse in the middle of the recoupling period in $\mathrm{S}$ but not in $\mathrm{S}_{0}$, which inverts the $\mathrm{S}$-L coherence. If that coherence decays completely before the inversion or saturation pulse, then there will be no difference between $\mathrm{S}$ and $\mathrm{S}_{0}$. $\mathrm{T}_{\mathrm{SL}}$ values are seen to be inversely proportional to $\mathrm{T}_{1 \mathrm{Q}}$ and to the square of the coupling strength. These power laws can be derived by analytical calculations for $\mathrm{L}=1 / 2$, see Appendix $\mathrm{C}$.

At $\mathrm{T}_{1 \mathrm{Q}}=1 / \omega_{\mathrm{SL}}, \mathrm{S}_{0}$ has a minimum for both coupling strengths, and the decrease in $\mathrm{T}_{\mathrm{SL}}$ for $\mathrm{S}$ starts to level off. For $\mathrm{T}_{1 \mathrm{Q}}>1 / \omega_{\mathrm{SL}}$, the $\mathrm{T}_{\mathrm{SL}}$ values for the $\mathrm{S}$ signal approach plateaus independent of $\mathrm{T}_{1 \mathrm{Q}}$. This is the standard regime with negligible effects of $\mathrm{T}_{1 \mathrm{Q}}$ on the REDOR decay. The heights of the plateaus are inversely proportional to the coupling strengths, as expected since a larger coupling strength will produce a faster initial decay (as part of the cosine oscillation) and thus a shorter decay constant $\mathrm{T}_{\mathrm{SL}}$.

Further, the plot shows that $T_{\mathrm{SL}}$ for $\mathrm{S}_{0}$ increases as $\mathrm{T}_{1 \mathrm{Q}}>1 / \omega_{\mathrm{SL}}$ increases and values for different coupling strengths merge on a line $\mathrm{T}_{\mathrm{SL}}=2 \mathrm{~T}_{1 \mathrm{Q}}$. Again, this power law has been derived for spin-1/2 in Appendix C. Qualitatively, one can say that for longer $\mathrm{T}_{1 \mathrm{Q}}$, a larger part of the S-L coherence can be refocused, independent of coupling strength, before it has relaxed, so $\mathrm{S}_{0}$ decays more slowly as $\mathrm{T}_{1 \mathrm{Q}}$ increases.

Different double- and single-quantum relaxation times. So far, we have assumed that the double-quantum and single-quantum relaxation rates are equal. In order to explore their independent effects, Fig. 7. 5a displays a contour plot of $\log \mathrm{T}_{\mathrm{SL}}{ }^{0}$ as a function of $\log \left(\mathrm{T}_{1 \mathrm{Q}}{ }^{\mathrm{SQ}}\right)$ and $\log \left(\mathrm{T}_{1 \mathrm{Q}}{ }^{\mathrm{DQ}}\right)$ for the $\mathrm{S}$ decay in REDOR for $\mathrm{L}=1$. Figure 7.5(b) shows the corresponding plot of $\log \mathrm{T}_{\mathrm{SL}}$ of the $\mathrm{S}_{0}$ intensity. The plots show that (i) ideal REDOR with long $\mathrm{T}_{\mathrm{SL}}{ }^{0}$ is recovered only if both $\mathrm{T}_{1 \mathrm{Q}}{ }^{\mathrm{SQ}}$ and $\mathrm{T}_{1 \mathrm{Q}}{ }^{\mathrm{DQ}}$ are long (upper right corner); (ii) self-decoupling 
(both $\mathrm{T}_{\mathrm{SL}}{ }^{0}$ and $\mathrm{T}_{\mathrm{SL}}$ are long) will occur if at least one relaxation time is short, and $\mathrm{T}_{1 \mathrm{Q}}{ }^{\mathrm{DQ}}$ is more effective at producing self-decoupling; (iii) if both $\mathrm{T}_{\mathrm{SL}}{ }^{0}$ and $\mathrm{T}_{\mathrm{SL}}$ are short, one of them must be between $1 /\left(2 \omega_{\mathrm{SL}}\right)$ and $3 / \omega_{\mathrm{SL}}$ and the other longer than $1 /\left(2 \omega_{\mathrm{SL}}\right)$.

As mentioned above, $\mathrm{T}_{1 \mathrm{Q}} \mathrm{SQ}$ and $\mathrm{T}_{1 \mathrm{Q}}{ }^{\mathrm{DQ}}$ are typically not very different. The dashed lines in Fig. 7. 5 delineate the expected range of $\mathrm{T}_{1 \mathrm{Q}}{ }^{\mathrm{SQ}} \approx \mathrm{T}_{1 \mathrm{Q}}{ }^{\mathrm{DQ}}$ values. The curves of $\log \mathrm{T}_{\mathrm{SL}}{ }^{0}$ and $\log \mathrm{T}_{\mathrm{SL}}$ as a function of $\log \left(\mathrm{T}_{1 \mathrm{Q}}\right)$ shown in Fig. 7. 4 are found along the diagonals $\left(\mathrm{T}_{1 \mathrm{Q}}{ }^{\mathrm{SQ}}\right.$ $=\mathrm{T}_{1 \mathrm{Q}}{ }^{\mathrm{DQ}}$ ) of the contour plots of Fig. 7.5, with a plateau in $\mathrm{T}_{\mathrm{SL}}{ }^{0}$ at large $\mathrm{T}_{1 \mathrm{Q}}{ }^{\mathrm{SQ}}=\mathrm{T}_{1 \mathrm{Q}}{ }^{\mathrm{DQ}}$ values and a minimum in $\mathrm{T}_{\mathrm{SL}}$ near the center of the plot. The behavior within the dashed lines is qualitatively similar as along the corresponding diagonal.

Heteronuclear evolution in the SPIDER experiment. In the SPIDER pulse sequence shown in Fig. 7. 1(b), the SPIDER S signal is obtained after applying irradiation on the Lspin, which destroys the $\mathrm{L}_{\mathrm{z}}$ terms in conjunction with the strong quadrupolar coupling. The dephasing of ${ }^{13} \mathrm{C}$ signal by adjacent quadrupolar nuclei is accomplished during the following recoupling period. The $\pi / 2$ pulse before the period of ${ }^{14} \mathrm{~N}$ irradiation stores one component of the ${ }^{13} \mathrm{C}$ magnetization along $\mathrm{z}$ so that it relaxes more slowly, while the second $\pi / 2$ pulse returns it to the transverse plane. In SPIDER $S_{0}$, there is no irradiation on the quadrupolar nucleus and the recoupling of ${ }^{13} \mathrm{C}-{ }^{14} \mathrm{~N}$ is refocused by the end of the second half recoupling period. The difference $\Delta \mathrm{S}=\mathrm{S}_{0}-\mathrm{S}$ plotted as a function of $\mathrm{Nt}_{\mathrm{r}}$ reveals the coupling strength between ${ }^{13} \mathrm{C}$ and the quadrupolar nucleus ${ }^{14} \mathrm{~N}$. In fact, this is not true when $\mathrm{T}_{1 \mathrm{Q}}$ is short. Our powder-averaged simulations with $\delta_{\mathrm{SL}}=2 \pi 1.5 \mathrm{kHz}$ shown in Fig. 7.6 indicate that only for $\mathrm{T}_{1 \mathrm{Q}}>100 \mathrm{~ms}$, it shows the expected difference between $\mathrm{S}_{0}$ and $\mathrm{S}$. The effects of $\mathrm{T}_{1 \mathrm{Q}}$ relaxation in SPIDER become significant already at longer $\mathrm{T}_{1 \mathrm{Q}}$ times than in REDOR. Most importantly, the heteronuclear evolution in SPIDER is effectively only half as fast as in REDOR [5]. In addition, the $\mathrm{S}_{0}$ signal decays faster in SPIDER than in REDOR due to $\mathrm{T}_{1 \mathrm{Q}}$ relaxation during the 0.8 -ms period for L-spin irradiation (which, of course, is applied only when $\mathrm{S}$ is measured, see Fig. 7. 1. As a result, shorter $T_{1 \mathrm{Q}}$ strongly reduces the $\Delta S=\mathrm{S}_{0}-\mathrm{S}$ intensity, especially when $\mathrm{T}_{1 \mathrm{Q}} \leqslant 1 \mathrm{~ms}$, where $\mathrm{S}_{0} \approx \mathrm{S}$; this is apparently the case for the 159 ppm and 56 ppm ${ }^{13} \mathrm{C}$ sites in Boc- $\operatorname{Arg}(Z)_{2}-\mathrm{OH}$, showing $\Delta \mathrm{S}=\mathrm{S}_{0}-\mathrm{S} \approx 0$ in Fig. 7. 11(b) below. 
The effects of $\mathrm{T}_{1 \mathrm{Q}}$ on the theoretical maximum $\Delta \mathrm{S}$ intensity that can be obtained by REDOR experiments for $\mathrm{L}=1 / 2,1$ and 3/2, and by SPIDER experiments for $\mathrm{L}=1$ are indicated in Fig. 7. 7. For very short $\mathrm{T}_{1 \mathrm{Q}}, \Delta \mathrm{S}$ remains vanishingly small due to selfdecoupling; for intermediate $\mathrm{T}_{1 \mathrm{Q}}, \Delta \mathrm{S}$ increases significantly as $\mathrm{T}_{1 \mathrm{Q}}$ increases. For long $\mathrm{T}_{1 \mathrm{Q}}>$ $100 / \delta, \Delta S$ asymptotically approaches its maximum value, which is 1 for $\mathrm{L}=1 / 2$ and $\mathrm{L}=3 / 2$, and $2 / 3$ for $L=1$.

Design of pulse sequences for $\mathbf{S}_{\mathbf{0 0}}$. The $\mathrm{T}_{1 \mathrm{Q}}$ relaxation effect during the $\mathrm{S}_{0}$ pulse sequence, see Fig. 7. 8(a), is significant due to the continuous recoupling of the SL interactions during each of the two halves of the pulse sequence. The phase

$$
\Phi_{S L}(t)=\int_{0}^{t} \omega_{S L}\left(t^{\prime}\right) d t^{\prime}
$$

keeps growing to $\mathrm{N} \Phi_{\mathrm{SL}}\left(\mathrm{t}_{\mathrm{r}} / 2\right)$ at $\mathrm{Nt}_{\mathrm{r}} / 2$. The two-spin coherence, which is proportional to $\sin \Phi_{\mathrm{SL}}(\mathrm{t})$, grows accordingly. The larger the S-L coherence, the larger will be the effect of $\mathrm{T}_{1 \mathrm{Q}}$ relaxation of the $\mathrm{L}$-component of that coherence. If generation of $\mathrm{S}-\mathrm{L}$ coherence could be avoided, $\mathrm{T}_{1 \mathrm{Q}}$ relaxation would have no effect.

This undesirable signal reduction can be minimized in a pulse sequence without significant long-time recoupling. The simplest such sequence would be a $\mathrm{Nt}_{\mathrm{r}} / 2$-- $\pi$-pulse -$\mathrm{Nt}_{\mathrm{r}} / 2$ Hahn-spin-echo sequence. Note that even here, the $\mathrm{S}-\mathrm{L}$ coherence is generated transiently, with the maximum phase $\Phi_{\mathrm{SL}}\left(\mathrm{t}_{\mathrm{r}} / 2\right)$ in the middle of each rotation period. More importantly, the signal from this sequence will not be directly comparable with $\mathrm{S}$ or $\mathrm{S}_{0}$, since those signals are obtained after many more $\pi$-pulses. Due to the effects of imperfect ${ }^{1} \mathrm{H}$ decoupling during the pulses, pulse flip-angle errors, resonance offset, etc., the decay of $\mathrm{S}_{0}$ is much faster than $\mathrm{T}_{2}$ relaxation.

Therefore, we designed pulse sequences with the same number of $180^{\circ}$ pulses as that for $S$ and $S_{0}$ but minimal $S-L$ recoupling. The most successful example is shown in Fig. 7. 8(b). It features two rotation-synchronized Hahn echoes, at the beginning and end. Around the center, $180^{\circ}$ pulses are applied every $\mathrm{t}_{\mathrm{r}} / 3$ for four rotation periods, so that at the center, 
$\Phi_{\mathrm{SL}}\left(\mathrm{Nt}_{\mathrm{r}} / 2\right)=0$. Due to the fast alternation of the sign of the effective frequency imposed by the relatively closely-spaced $180^{\circ}$ pulses, the phase $\Phi_{\mathrm{SL}}$ remains small throughout the whole recoupling period. Evolutions of the phase $\Phi_{\mathrm{SL}}$ under REAPDOR or SPIDER $\mathrm{S}$ and $\mathrm{S}_{0}$ pulse sequences as well as the newly designed $\mathrm{S}_{00}$ sequence, calculated using the SIMPSON program [20], are shown in Fig. 7. 8(c). While for REDOR $S_{0}, \Phi_{\mathrm{SL}}$ reaches a large value of $8 \Phi_{\mathrm{SL}}\left(\mathrm{t}_{\mathrm{r}} / 2\right)$ at $\mathrm{Nt}_{\mathrm{r}} / 2, \Phi_{\mathrm{SL}}$ is seen to be always comparable or smaller than $1.55 \Phi_{\mathrm{SL}}\left(\mathrm{t}_{\mathrm{r}} / 2\right)$ during the $S_{00}$ pulse sequence; for reference, free evolution gives a maximum of $1.0 \Phi_{\mathrm{SL}}\left(\mathrm{t}_{\mathrm{r}} / 2\right)$. Independent EXORCYCLEs [21] of the first and last $180^{\circ}$ pulses (in the Hahn-echo part of the sequence) were employed. The same phase sequence and phase cycle were applied in the $S_{0}$ pulse sequence, which actually appeared to increase the $S_{0}$ intensity compared to an incomplete xy phase sequence on all fourteen $180^{\circ}$ pulses. We also designed and tested many other pulse sequences with some smaller pulse spacings than shown in Fig. 7. 8(b), but they did not work as well as the one presented here, in particular being more narrow-band.

The signal obtained with minimum recoupling is termed $S_{00}$. The $S_{00}$ and $S_{0}$ spectra match well near resonance (within $\pm 1 \mathrm{kHz}$ ). In our experiments, the match of the $\mathrm{S}_{00}$ and $\mathrm{S}_{0}$ spectra was found to remain good (within $\pm 4 \%$ ) down to $-12 \mathrm{kHz}$ downfield from resonance, while it degraded markedly for offsets $>+1 \mathrm{kHz}$ upfield from resonance. The reason for this asymmetry, which was similar for different $\mathrm{xy}-8$ sequences (e.g. $\mathrm{x} \mathrm{y} \mathrm{x} \mathrm{y;} \mathrm{x}-\mathrm{y}$ $\mathrm{x}-\mathrm{y}$; and $-\mathrm{x}-\mathrm{y}-\mathrm{x}-\mathrm{y}$ ), remains unclear. The $\mathrm{S}_{00}$ sequence produced reduced $\mathrm{C}-\mathrm{H}$ dephasing, resulting in $\sim 10 \%$ higher signals of $\mathrm{CH}$ groups. This was compensated empirically by $6 \mu \mathrm{s}$ of dipolar dephasing before detection, for all samples measured.

Table 1 provides an overview of the intensities of $S, S_{0}$, and $S_{00}$ for various $T_{1 Q}$ regimes. The entries are based on the curves in Figs. 7.3, 7.6 and 7.7 (for $\mathrm{S}, \mathrm{S}_{0}$, and $\Delta \mathrm{S}$ ) and on the experimental observations for $\mathrm{S}_{00}$. In the regime with negligible relaxation, $\mathrm{T}_{1 \mathrm{Q}}>$ $100 / \delta$, we have $S_{00}=S_{0}$, otherwise, $S_{00}>S_{0}$. Finally, for $T_{1 Q}<<1 / \delta$, self-decoupling is reached, where there is hardly any heteronuclear evolution before the spin state of the quadrupolar nucleus relaxes and therefore no ${ }^{13} \mathrm{C}-{ }^{14} \mathrm{~N}$ correlation can be detected. $\Delta \mathrm{S}_{0}$ represents the improvement of signal intensity made by using the new pulse sequence. However, directly obtaining the full $\Delta S_{\text {tot }}$ in one spectrum by combining the new $S_{00}$ pulse sequence with SPIDER dephasing in S is experimentally very challenging for demanding 
samples like natural organic matter, because the artifacts from a few percent of mismatch between $S_{00}$ and $S_{0}$ will be comparable to the desired signal $\Delta S_{\text {tot }}$.

$\mathbf{T}_{1 Q^{-}}$-enhanced, $\omega_{\mathrm{r}}$-dependent $\mathbf{T}_{2 \mathrm{~S}}$, MAS relaxation. Even during free MAS without recoupling pulses, the effect of $\mathrm{T}_{1 \mathrm{Q}}$ relaxation on the heterogeneous evolution may be detectable. During the course of each rotation period $t_{r}, S_{y} L_{z}$ coherence is generated at the expense of $S_{x}$ and relaxes towards zero. By the end of the rotation period, the two-spin coherence is reconverted into magnetization, but less so if the two-spin coherence has been depleted by relaxation. This speeds up the effective $\mathrm{T}_{2}$ relaxation of the observed $\mathrm{S}$-spin. The evolution at the beginning of each rotation period is the same (in the quasistatic regime) but is terminated the earlier, the shorter the rotation period, since the initial state must be recovered at the end of the rotation period. Therefore, the longer the rotation period, the greater the two-spin coherence will become, see Fig. 7.9 (a) (b). As a result, the effective $\mathrm{T}_{2}$ relaxation time of the $\mathrm{S}$-spin $\left(\mathrm{T}_{2 \mathrm{~S}, \mathrm{MAS}}\right.$ ) will decrease markedly with increasing $\mathrm{t}_{\mathrm{r}}$ and decreasing spinning frequency.

The dependence of $\mathrm{T}_{2 S}$, MAS on the spinning-frequency $\omega_{\mathrm{r}}$, relaxation time $\mathrm{T}_{1 \mathrm{Q}}$, and coupling constant $\delta_{\text {SL }}$ can be predicted using numerical simulation. The time-dependent frequency during free MAS can be written as:

$$
\begin{aligned}
\omega_{\mathrm{SL}}(\mathrm{t})= & \mathrm{C}_{1} \cos \left(\gamma+\omega_{\mathrm{r}} \mathrm{t}\right)+\mathrm{C}_{2} \cos \left(2 \gamma+2 \omega_{\mathrm{r}} \mathrm{t}\right) \\
& +\mathrm{S}_{1} \sin \left(\gamma+\omega_{\mathrm{r}} \mathrm{t}\right)+\mathrm{S}_{2} \sin \left(2 \gamma+2 \omega_{\mathrm{r}} \mathrm{t}\right)
\end{aligned}
$$

For a spin-pair dipolar coupling, $\mathrm{S}_{1}=\mathrm{S}_{2}=0$, [22] and

$$
\begin{aligned}
& \mathrm{C}_{1}=-\delta_{S L} \sqrt{2} / 2 \sin (2 \beta) \\
& \mathrm{C}_{2}=\delta_{\mathrm{SL}} / 2 \sin ^{2} \beta .
\end{aligned}
$$

We use $\omega_{S L}(t)$ instead of the constant $\omega_{S L}$ in Eq. (7.8) and evaluate the evolution numerically as outlined in Appendix A, Eq. (7.A4). Powder averaging is performed by 
adding curves for $\beta=0^{\circ}, \cdots, 90^{\circ}$ with $\sin \beta$ weighting. The effect of $\gamma$ on the simulations is minimal, since all values of $\gamma+\omega_{\mathrm{r}} \mathrm{t}$ are scanned in the course of each rotation period.

Parameter dependencies of $\mathbf{T}_{2 S}$, MAS. At a fixed spinning frequency $(8 \mathrm{kHz})$, the $\mathrm{T}_{2 \mathrm{~S} \text {, MAS }-}$ relaxation time decreases with decreasing $\mathrm{T}_{1 \mathrm{Q}}$ and increases when $\mathrm{T}_{1 \mathrm{Q}}<20 \mu$ s (see simulations for $\mathrm{L}=1$ in Fig. 7. 9(c)), due to the loss of heteronuclear coherence. Figure 7. 10(d) illustrates that $T_{2 S, M A S}$ increases with spinning frequency for constant $T_{1 Q}$. This effect is more pronounced when $\mathrm{T}_{1 \mathrm{Q}}$ is relatively long. However, when $\mathrm{T}_{1 \mathrm{Q}}$ is smaller than $3 \mu \mathrm{s}$, $\mathrm{T}_{2 \mathrm{~S}, \mathrm{MAS}}$ is almost independent of spinning frequency, increasing by only $0.1 \%$ from $3 \mathrm{kHz}$ to $12 \mathrm{kHz}$ : The two-spin coherence $\mathrm{S}_{\mathrm{y}} \mathrm{L}_{\mathrm{z}}$ relaxes so fast that it does not accumulate significantly during one rotation period, even at low spinning speed, and therefore, it shows little dependence on the spinning speed. The effect of $\mathrm{T}_{1 \mathrm{Q}}$ on $\mathrm{T}_{2 \mathrm{~S} \text {,MAS }}$ for different spinning speeds is shown in Fig. 7. 9(e). Generally, in the short- $\mathrm{T}_{1 \mathrm{Q}}$ regime, $\mathrm{T}_{2 \mathrm{~S}, \mathrm{MAS}}$ decreases as $\mathrm{T}_{1 \mathrm{Q}}$ increases, due to the increased coupling generating large two-spin coherence term $\mathrm{S}_{\mathrm{y}} \mathrm{L}_{\mathrm{z}}$ combined with fast relaxation of $\mathrm{L}_{\mathrm{z}}$; in the long- $\mathrm{T}_{1 \mathrm{Q}}$ regime of spin $\mathrm{L}, \mathrm{T}_{2 \mathrm{~S}, \mathrm{MAS}}$ increases with $\mathrm{T}_{1 \mathrm{Q}}$, due to the slow-down of the decay of the two-spin coherence term $\mathrm{S}_{\mathrm{y}} \mathrm{L}_{\mathrm{Z}}$. At a higher spinning speed, the $\mathrm{T}_{2 \mathrm{~S}, \mathrm{MAS}}$ minimum value is larger and appears at a smaller $\mathrm{T}_{1 \mathrm{Q}}$ than that for slower spinning.

The $\omega_{\mathrm{SL}}$ dependence of $\mathrm{T}_{2 \mathrm{~S} \text {, MAS }}$ relaxation was also explored in simulations for a fixed spinning speed of $2 \pi 6 \mathrm{kHz}$, see Fig. 7. 9. Figure 7.11 (a) shows the $T_{1 \mathrm{Q}}$-dependence of $\mathrm{T}_{2 \mathrm{~S} \text {, MAS }}$ for coupling strengths between $2 \pi 0.1 \mathrm{kHz}$ and $2 \pi 10 \mathrm{kHz}$. The curves of $\log \mathrm{T}_{2 \mathrm{~S}}$, MAS vs. $\log T_{1 Q}$ have approximately the same shape, with minima fixed at $T_{1 Q}=1 /(2 \pi 6 \mathrm{kHz})$ $=27 \mu \mathrm{s}$ for $\omega_{\mathrm{SL}}<\omega_{\mathrm{r}}$, see Figure $7.10(\mathrm{a}, \mathrm{b})$. This is due to the evolution being mainly modulated by $\omega_{\mathrm{r}}$ when $\omega_{\mathrm{SL}}<<\omega_{\mathrm{r}}$. As a result, the largest effects of $\mathrm{T}_{1 \mathrm{Q}}$ relaxation are observed for smaller $\mathrm{T}_{1 \mathrm{Q}}$ values in free MAS (with $\omega_{\mathrm{r}}$ modulation) than in recoupling experiments (with $\omega_{\mathrm{SL}}$ modulation), since in practice usually $\omega_{\mathrm{SL}}<\omega_{\mathrm{r}}$. For $\omega_{\mathrm{SL}}=2 \pi 10 \mathrm{kHz}$ $>\omega_{\mathrm{r}}$, a slight horizontal shift of the minimum is observed: When $\omega_{\mathrm{SL}}>>\omega_{\mathrm{r}}$, the evolution of the two-spin coherence is modulated by $\omega_{\mathrm{SL}}$ and the $\mathrm{T}_{2 \mathrm{~S} \text {, MAS }}$ minimum position will be $\omega_{\mathrm{SL}^{-}}$ dependent. The depth of the minimum depends strongly on the coupling strength $v_{\mathrm{SL}}=$ 
$\omega_{\mathrm{SL}} / 2 \pi$. Linear fitting shows an inverse-square dependence of $\mathrm{T}_{2 \mathrm{~S}, \mathrm{MAS}}$ on $v_{\mathrm{SL}}, \mathrm{T}_{2 \min }=10^{1.27}$ $\mathrm{kHz}\left(1 / v_{\mathrm{SL}}\right)^{2}$, see Fig. 7. 10 (b).

\section{Experimental Results and Discussion}

"Missing signals" in ${ }^{13} \mathbf{C}\left\{{ }^{14} \mathbf{N}\right\}$ SPIDER NMR. CP/TOSS and SPIDER spectra of Boc-Arg$\mathrm{OH}$ and Boc- $\operatorname{Arg}(\mathrm{Z})_{2}-\mathrm{OH}$ are shown in Fig. 7. 11(a) and (b), respectively. In the $\triangle \mathrm{S}$ SPIDER spectrum of Boc-Arg- $\mathrm{OH}$, all the expected signals of $\mathrm{C}$ bonded to $\mathrm{N}$ are observed. By contrast, the chemically similar $\mathrm{Boc}-\mathrm{Arg}(\mathrm{Z})_{2}-\mathrm{OH}$ does not produce the expected $\Delta \mathrm{S}$ peaks for the $\mathrm{C}=\mathrm{O}$ and $\mathrm{C}-\mathrm{H}$ carbons bonded to the "backbone" N. A two-fold signal reduction was also observed in the SPIDER $\triangle \mathrm{S}$ spectrum of chitin, an N-acetylated polysaccharide that forms the exoskeleton of arthropods, see Fig. 7. 5 of ref. (7). In the following, we prove that these signal losses are due to relaxation of the ${ }^{14} \mathrm{~N}$ spin state during the $\mathrm{S}_{0}$ pulse sequence, and demonstrate the predicted unusual $\mathrm{T}_{2 \mathrm{~S}}$, MAS behavior for these same carbon sites.

Measurement of $\Delta \mathbf{S}_{\mathbf{0}}$ from $\mathbf{S}_{\mathbf{0 0}}$ and $S_{\mathbf{0}}$ spectra. $S_{00}$ spectra of Boc-Arg-OH and Boc$\operatorname{Arg}(\mathrm{Z})_{2}-\mathrm{OH}$ with minimal recoupling are displayed in Fig. 7. 12, measured with the new pulse sequence shown in Fig. 7. 8(b). They are compared with $S_{0}$ spectra obtained using the matching pulse sequence of Fig. 7. 8(a). The difference $\Delta \mathrm{S}_{0}=\mathrm{S}_{00}-\mathrm{S}_{0}$ reveals signals loss due to $T_{1 Q}$ relaxation during the refocused recoupling. The intensity of $\Delta S_{0}$ matches the $S_{0}$ intensity loss in the SPIDER experiments, in particular for the bands of $\mathrm{Boc}-\operatorname{Arg}(\mathrm{Z})_{2}-\mathrm{OH}$ at $159 \mathrm{ppm}$ and $56 \mathrm{ppm}$, which are due to the carbons bonded to the "backbone" nitrogen. This provides an experimental confirmation of our hypothesis that the reduced $\mathrm{S}_{0}$ intensity in the SPIDER spectra is due to transient recoupling to a ${ }^{14} \mathrm{~N}$ with short $\mathrm{T}_{1 \mathrm{Q}}$.

The observed $\Delta \mathrm{S}_{0} / \mathrm{S}_{00}$ and $\Delta \mathrm{S} / \mathrm{S}_{0}$ values enable us to estimate $\mathrm{T}_{1 \mathrm{Q}}$, based on the curves of Fig. 7. 6. The small $\Delta \mathrm{S}$ signal at $159 \mathrm{ppm}$ and $56 \mathrm{ppm}$ indicates that $\mathrm{T}_{1 \mathrm{Q}}<0.3 \mathrm{~ms}$, according to Fig. 7. 6(c). The experimentally observed significant decay of $S_{0}$ shows that the self-decoupling regime has not been reached, $\mathrm{T}_{1 \mathrm{Q}}>0.3 \mathrm{~ms}$, according to Fig. 7. 6(b).

The $\mathrm{S}_{00}, \mathrm{~S}_{0}$, and $\Delta \mathrm{S}_{0}$ spectra of chitin, an $\mathrm{N}$-acetylated polysaccharide, and of the oligopeptide, $(\mathrm{POG})_{10}$, are shown in Fig. 7. 13. For chitin, the considerable $\Delta \mathrm{S}_{0}$ intensity for 
the signals of the two carbons bonded to $\mathrm{N}$ proves that the weak SPIDER $\Delta \mathrm{S}$ signal found in ref. [5] is caused by the reduced intensity of $\mathrm{S}_{0}$. The observed dephasing, $\Delta \mathrm{S} / \mathrm{S}_{0} \sim 0.25$ and $\Delta \mathrm{S}_{0} / \mathrm{S}_{00} \sim 0.25$, indicates that $0.3 \mathrm{~ms}<\mathrm{T}_{1 \mathrm{Q}}<1 \mathrm{~ms}$. For $(\mathrm{POG})_{10}$, intense signals of all carbons connected to $\mathrm{N}$ show up in the $\Delta \mathrm{S}_{0}$ spectrum, indicative of short $\mathrm{T}_{1 \mathrm{Q}}$ between 0.2 and $1 \mathrm{~ms}$ for proline and hydroxyproline $\mathrm{N}$ in the peptide.

For carbon sites close to quadrupolar spins with sufficiently long $\mathrm{T}_{1 \mathrm{Q}}>10 \mathrm{~ms}$, recoupling techniques such as SPIDER, REAPDOR or TRAPDOR give the expected results; ${ }^{13} \mathrm{C}$ sites close to ${ }^{14} \mathrm{~N}$ nuclei in Boc-Arg-OH are examples. Nevertheless, even for these carbons, the non-zero $\Delta \mathrm{S}_{0}$ intensity also indicates a slight intensity reduction of $\mathrm{S}_{0}$. Adaptations of the new $S_{00}$ pulse sequence for SPIDER, REAPDOR, or RIDER experiments would facilitate applications of these methods to systems with short $\mathrm{T}_{1 \mathrm{Q}}$ relaxation times. The challenge consists in matching $\mathrm{S}_{00}$ and $\mathrm{S}$ pulse sequences well enough to make the artifact difference signals much smaller than those of the nitrogen-bonded carbons.

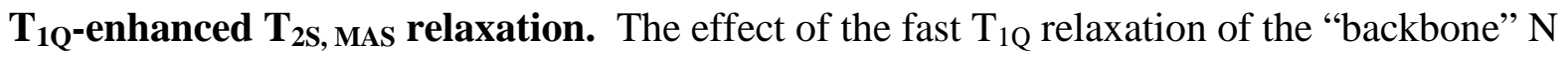

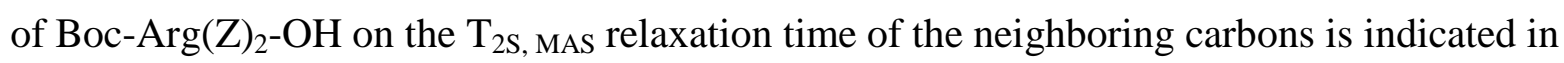
Fig. 7. 14, which shows the dependence of $T_{2 S, M A S}$ on $\omega_{\mathrm{r}}$ for the nonprotonated carbons. The curves show that the $T_{2 S}$, MAS values of the carbons bonded to $\mathrm{N}$ increase as spinning speed increases, most prominently for the 159-ppm carbon, which also showed the largest $\Delta \mathrm{S}_{0}$ signal. At faster MAS, the rise of the curves becomes less obvious due to imperfect ${ }^{1} \mathrm{H}$ decoupling resulting from interference by MAS. The measured data points are compared with simulated curves, and the fits give good estimates of the ${ }^{14} \mathrm{~N} \mathrm{~T}_{1 \mathrm{Q}}$ relaxation times. The ${ }^{14} \mathrm{~N}$ bonded to the "backbone" carbon with a chemical shift of $159 \mathrm{ppm}$ has a short $\mathrm{T}_{1 \mathrm{Q}}$ relaxation time of ca. $0.2 \mathrm{~ms}$, while the other ${ }^{14} \mathrm{~N}$ sites have $\mathrm{T}_{1 \mathrm{Q}}$ values of $\sim 2 \mathrm{~ms}$. In the fit of the signal of the carbon resonating at $161 \mathrm{ppm}$, it was assumed that the three ${ }^{14} \mathrm{~N}$ to which it is bonded have the same $\mathrm{T}_{1 \mathrm{Q}}$ of $\sim 2 \mathrm{~ms}$, giving an apparent $\mathrm{T}_{1 \mathrm{Q}}{ }^{\text {app }}=0.67 \mathrm{~ms}$ based on $1 / \mathrm{T}_{1 \mathrm{Q}}{ }^{\text {app }} \approx 3 \times 1 /(2 \mathrm{~ms})$.

Dynamics driving $\mathbf{T}_{1 \mathrm{Q}}$-relaxation. The short $\mathrm{T}_{1 \mathrm{Q}}$ of the nitrogen in the $\mathrm{N}$-acetyl side groups of chitin is not too surprising, given that torsional fluctuations can rock the side group. In 
collagen and related peptides, motions of proline rings have been known to shorten $\mathrm{T}_{1 \mathrm{C}}$.[23] The mechanism of relaxation in $\operatorname{Boc}-\mathrm{Arg}(\mathrm{Z})_{2}-\mathrm{OH}$ is less obvious. In order to probe the MHz-rate motions that drive $\mathrm{T}_{1 \mathrm{Q}}$ of ${ }^{14} \mathrm{~N}$, we have measured the longitudinal $\mathrm{T}_{1}$ relaxation times of all resolved carbon sites in Boc-Arg-OH and Boc- $\operatorname{Arg}(\mathrm{Z})_{2}-\mathrm{OH}$, see Fig. 7. 15. The values show that the $\mathrm{T}_{1 \mathrm{C}}$ values of sites near the $-\mathrm{COOH}$ group in the "backbone" of these two compounds are quite different, suggesting some changes around $\mathrm{COOH}$ in $\mathrm{Boc}-\operatorname{Arg}(\mathrm{Z})_{2^{-}}$ $\mathrm{OH}$.

It is instructive to compare the measured ${ }^{13} \mathrm{C}$ and estimated ${ }^{14} \mathrm{~N} \mathrm{~T}_{1}$ relaxation times for neighboring sites. An approximate relationship can be obtained based on the following assumptions, which enable quantitative calculations [24]: (i) Dipolar interaction between ${ }^{13} \mathrm{C}$ and ${ }^{1} \mathrm{H}$ is the main spin-lattice relaxation mechanism for ${ }^{13} \mathrm{C}$. (ii) Quadrupolar coupling provides the dominant relaxation mechanism for ${ }^{14} \mathrm{~N}$. (iii) The reorientational correlation times $\tau_{\mathrm{c}}$ are identical for both ${ }^{13} \mathrm{C}$ and ${ }^{14} \mathrm{~N}$. (iv) The molecule undergoes isotropic motions. In the fast motion limit, the relaxation rate of ${ }^{13} \mathrm{C}$ due to $\mathrm{C}-\mathrm{H}$ dipolar coupling can be given as:

$$
1 / \mathrm{T}_{1 \mathrm{C}}=(4 / 3) \gamma_{13 \mathrm{C}}^{2} \gamma_{14 \mathrm{~N}}^{2} h \mathrm{~S}(\mathrm{~S}+1) \mathrm{r}_{\mathrm{C}-\mathrm{H}}{ }^{-6} \tau_{\mathrm{c}}
$$

and the quadrupolar relaxation rate of ${ }^{14} \mathrm{~N}$ is

$$
1 / \mathrm{T}_{1 \mathrm{Q}}=(3 / 8)\left(\mathrm{e}^{2} \mathrm{qQ} /\right)^{2} \tau_{\mathrm{c}}
$$

With $\mathrm{r}_{\mathrm{C}-\mathrm{H}}=1.09 \AA$ and $\mathrm{e}^{2} \mathrm{qQ} / \mathrm{h}=3 \mathrm{MHz}$, we find

$$
\mathrm{T}_{1 \mathrm{C}}=6200 \mathrm{~T}_{1 \mathrm{Q}}
$$

From the experimentally measured $\mathrm{T}_{1 \mathrm{C}}$ value of $11 \mathrm{~s}$ for $\mathrm{C}_{\square}$ in $\mathrm{N}-\mathrm{t}-\mathrm{Boc}-\mathrm{Arg}(\mathrm{Z})_{2}-\mathrm{OH}, \mathrm{Eq}$. (7.19) yields an estimated ${ }^{14} \mathrm{~N} \mathrm{~T}_{1 \mathrm{Q}}$ of $1.8 \mathrm{~ms}$, within an order of magnitude from the $0.2-\mathrm{ms}$ $\mathrm{T}_{1 \mathrm{Q}}$ value estimated from $\mathrm{S}_{0}$ and $\mathrm{T}_{2 \mathrm{~S}, \mathrm{MAS}}$ data.

In order to check for fast large-amplitude motions, the CSA powder patterns of the $\mathrm{C}=\mathrm{O}$ carbons were measured by SUPER [25] experiments in both Boc-Arg-OH and Boc- 
$\operatorname{Arg}(\mathrm{Z})_{2}-\mathrm{OH}$. The powder patterns for the $\mathrm{CO}$ sites in Fig. 7. 16 do not exhibit evidence of large amplitude motions. The chemical shift anisotropies determined from these spectra are summarized in Table 2. Comparison with literature CSA data of carboxyl groups[26] suggests that the $-\mathrm{COO}$ group in $\mathrm{Boc}-\mathrm{Arg}(\mathrm{Z})_{2}-\mathrm{OH}$ is protonated, while that in Boc-Arg-OH is deprotonated. We suspect that the NH proton in the "backbone" undergoes some motion, since we only observed two protonated $\mathrm{N}$ in the ${ }^{15} \mathrm{~N}$ spectrum (not shown), while there are three protonated sites in the structure of $\mathrm{Boc}-\operatorname{Arg}(\mathrm{Z})_{2}-\mathrm{OH}$. It is interesting to note that investigations of solid state proton exchange in a variety of organic compounds have also suggested that fast quadrupolar relaxation of ${ }^{14} \mathrm{~N}$ is induced by fluctuations in its electric field gradient that accompany proton exchange. [27]

$\mathbf{T}_{1 \mathrm{Q}}$ effects in J-coupled systems. This paper has focused on effects of $\mathrm{T}_{1 \mathrm{Q}}$ relaxation of the heteronucleus under dipolar recoupling and under free MAS. As briefly indicated above, the effect analogous to that with dipolar recoupling occurs during "free MAS" under the influence of a heteronuclear J-coupling [8]. In this case, the simulations for S dephasing with a single coupling frequency $\omega_{\mathrm{SL}}$ (see Figs. 3(a) and $\mathrm{S} 1$ (a)) are applicable. The $\mathrm{S}_{0}$ experiment corresponds exactly to a Hahn-echo measurement of $\mathrm{T}_{2}$, where the sign of the heteronuclear $\mathrm{J}$-coupling is effectively inverted by a $180^{\circ}$ pulse at $\tau=\mathrm{Nt}_{\mathrm{r}} / 2$. Thus, the single- $\omega_{\mathrm{SL}} \mathrm{S}_{0}$ decay curves as shown in Figs. 3(b) and S1(b), including some slight oscillations, are expected to be observed in $\mathrm{T}_{2}$ measurements of an S-nucleus J-coupled to a quadrupolar L-nucleus with short $\mathrm{T}_{1 \mathrm{Q}}$.

In practice, J-couplings in the $1-\mathrm{kHz}$ range are common for atoms with many electrons, i.e. for nucleides of higher mass. These have weak heteronuclear dipolar couplings due to the relatively large internuclear distances resulting from large atomic radii, but relatively large J-couplings. In ${ }^{125} \mathrm{Te}(\mathrm{S}=1 / 2) \mathrm{NMR}$ of $\mathrm{Sb}_{2} \mathrm{Te}_{3}$, we have indeed observed shortened $\mathrm{T}_{2, \mathrm{Te}, \mathrm{MAS}} \approx 0.4 \mathrm{~ms}$ compared to $\mathrm{Te}$ in PbTe or $\mathrm{GeTe}$, together with a short $\mathrm{T}_{1 \mathrm{Q}, \mathrm{Sb}}$, MAS $\approx 0.5 \mathrm{~ms}$ for the central transition of ${ }^{121} \mathrm{Sb}(\mathrm{L}=5 / 2)$.

Unpaired electrons. The dipolar coupling of nuclear spins with unpaired electrons of short longitudinal relaxation time or fast longitudinal spin exchange is another case where multi- 
spin coherence is generated by a coupling, decays fast due to relaxation or spin exchange of the (electron) L-spin, and shortens the $T_{2, \text { MAS }}$ of the observed nucleus $\mathrm{S}$. The quantitative analysis of this effect, where the couplings can be much larger than considered here $\left(>\omega_{\mathrm{r}}\right.$, while we implicitly assumed $\omega_{\mathrm{SL}}<\omega_{\mathrm{r}}$ ) and must be described by more complex interactions[28] is beyond the scope of this article.

\section{Conclusions}

The effects of fast longitudinal relaxation of a quadrupolar nucleus coupled to the observed spin-1/2 nucleus have been studied theoretically and experimentally. Theoretical analysis and simulations have shown that short $\mathrm{T}_{1 \mathrm{Q}}$ can cause significant reduction or even disappearance of $\Delta S$ signals in experiments like REDOR and SPIDER. In order to demonstrate and minimize the effect, a new pulse sequence with minimal recoupling has been designed and shown to produce an improved reference signal $\mathrm{S}_{00}$. A significant difference signal $\Delta S_{0}=S_{00}-S_{0}$ proves coupling to a heteronucleus with $T_{1 Q} \sim 0.3 \mathrm{~ms}$. Applications to a molecular crystal, to an $\mathrm{N}$-acetylated polysaccharide, and to a collagenmimetic oligopeptide show that the effect is quite common. Furthermore, studies of $\mathrm{T}_{1 \mathrm{Q}^{-}}$ enhanced, $\omega_{\mathrm{r}}$-dependent $\mathrm{T}_{2 S}$, MAS relaxation under free MAS without recoupling have shown both theoretically and experimentally that short $\mathrm{T}_{1 \mathrm{Q}}(5-200 \mu \mathrm{s})$ of the coupled

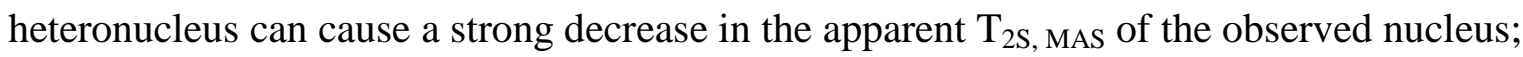

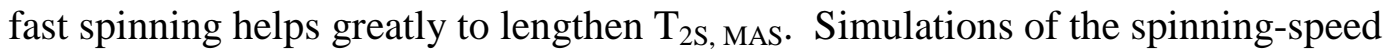
dependence provide good estimates of $\mathrm{T}_{1 \mathrm{Q}}$. Finally, possible reasons for the short $\mathrm{T}_{1 \mathrm{Q}}$ values of ${ }^{14} \mathrm{~N}$ in the studied compounds have been explored.

\section{Materials and Methods}

Samples. Arginine derivatives, $\mathrm{N}_{\alpha^{-}}$(tert-butoxycarbonyl)-L-arginine (Boc-Arg-OH) and $\mathrm{N}_{\alpha^{-}}$ Boc- $\mathrm{N}_{\delta}, \mathrm{N}_{\omega}$-di-Z-L-arginine (Boc-Arg $(\mathrm{Z})_{2}-\mathrm{OH}$ ), were obtained from Sigma-Aldrich. Boc$\operatorname{Arg}(\mathrm{Z})_{2}-\mathrm{OH}$ was $98 \%$ pure, and Boc-Arg-OH contained 5-10\% n-butanol. Chitin (poly (Nacetyl-1, 4- $\beta$-D-glucopyranosamine) was purchased from Fluka. (POG) 10 was purchased from Peptide Institute, Japan. All samples were used without further treatment. 
NMR parameters. The NMR experiments were performed using a Bruker DSX400 spectrometer at $400 \mathrm{MHz}$ for ${ }^{1} \mathrm{H}, 100 \mathrm{MHz}$ for ${ }^{13} \mathrm{C}$, and $29 \mathrm{MHz}$ for ${ }^{14} \mathrm{~N}$. A Bruker 4-mm triple-resonance probe head was used for the SPIDER experiments at a 5-kHz spinning frequency, with ca. $56 \mathrm{kHz}{ }^{1} \mathrm{H}$ decoupling power, which is increased to $63 \mathrm{kHz}$ during ${ }^{13} \mathrm{C} \pi$ pulses. The ${ }^{14} \mathrm{~N}$ radio-frequency field strength was $\gamma_{\mathrm{N}} \mathrm{B}_{1} \approx 2 \pi 30 \mathrm{kHz}$, and the ${ }^{13} \mathrm{C} 180^{\circ}$ pulse length was $8.1 \mu \mathrm{s}$. The recoupled dipolar evolution consisted of eight rotation periods (1.6 $\mathrm{ms}$ ), and the ${ }^{14} \mathrm{~N}$ irradiation time of four rotation periods. The 5-kHz MAS experiments using the pulse sequences shown in Fig. 7.8 (a) and (b) for measuring the regular and an improved reference signal $S_{0}$ and $S_{00}$, respectively, and $\Delta S_{0}$ were performed with a 4-mm probe head in double resonance mode. All experiments were performed with a cross polarization $(\mathrm{CP})$ contact time of $1 \mathrm{~ms}$ and a recycle delay of $2 \mathrm{~s}$. In measurements of $\mathrm{S}_{0}$ and $\mathrm{S}_{00}$, the ${ }^{13} \mathrm{C}$ irradiation frequency "on-resonance" was set near protonated carbon resonances during the ${ }^{13} \mathrm{C} 180^{\circ}$ pulse train and near those of non-protonated carbon resonances during detection. The gated decoupling time before detection in measuring $S_{00}$ was $6 \mu$ s.

SUPER experiments were used to obtain ${ }^{13} \mathrm{C}$ CSA powder patterns in the arginine derivatives. These experiments were carried out at a spinning speed of $5 \mathrm{kHz}$. The ${ }^{13} \mathrm{C}$ field strength of the $360^{\circ}$ pulses was $62.5 \mathrm{kHz}$ and the number of $\mathrm{t}_{1}$ increments was 96 , with an acquisition time of $5 \mathrm{~ms}$.

$\mathrm{T}_{1}$ and $\mathrm{T}_{2}$ relaxation time measurements were made after 1-ms cross polarization time, using a z-filter and Hahn echo, respectively. TOSS was used before and TPPM ${ }^{1} \mathrm{H}$ decoupling during detection.

\section{Acknowledgements}

This work was supported by the U.S. Department of Energy - Basic Energy Sciences under Contract No. DE-AC02-07CH11358. We thank M. Hong and Y. Zhang for making the $(\mathrm{POG})_{10}$ sample available.

\section{Appendices}

Appendix A. Free heteronuclear evolution with $\mathbf{T}_{1 \mathrm{Q}}$ relaxation, and numerical calculation 
Heteronuclear evolution with longitudinal L-spin relaxation is governed by the von Neumann equation with relaxation, $\mathrm{d} \rho / \mathrm{dt}=-\mathrm{i} / \mathrm{h}\left[\mathrm{H}_{\mathrm{SL}}, \rho\right]+\underline{\mathrm{R}} \rho$, Eq. (7.6), where the Redfield supermatrix $\underline{\mathrm{R}}$ acts only on the L-spin part of the density matrix; its effect is given by the relaxation exchange matrix $\mathrm{W}$ according to Eq. (7.2). Inserting the density matrix for $\mathrm{L}=1$, $\rho(t)=S_{x} \operatorname{diag}\left(p_{x, 1}, p_{x, 0}, p_{x,-1}\right)+S_{y} \operatorname{diag}\left(p_{y, 1}, p_{y, 0}, p_{y,-1}\right)$, into Eq. (7.6) gives

$$
\begin{aligned}
\mathrm{d} \rho / \mathrm{dt}= & \mathrm{S}_{\mathrm{y}}\left\{\omega_{\mathrm{SL}} \operatorname{diag}\left(\mathrm{p}_{\mathrm{x}, 1}, 0,-\mathrm{p}_{\mathrm{x},-1}\right)+\operatorname{diag}\left(\underline{\mathrm{W}}\left(\mathrm{p}_{\mathrm{y}, 1}, \mathrm{p}_{\mathrm{y}, 0}, \mathrm{p}_{\mathrm{y},-1}\right)\right)\right\}+ \\
& \mathrm{S}_{\mathrm{x}}\left\{-\omega_{\mathrm{SL}} \operatorname{diag}\left(\mathrm{p}_{\mathrm{y}, 1}, 0,-\mathrm{p}_{\mathrm{y},-1}\right)+\operatorname{diag}\left(\underline{\mathrm{W}}\left(\mathrm{p}_{\mathrm{x}, 1}, \mathrm{p}_{\mathrm{x}, 0}, \mathrm{p}_{\mathrm{x},-1}\right)\right)\right\}
\end{aligned}
$$

This can be set equal to the standard expression for the time derivative of $\rho(t)$,

$$
\begin{aligned}
\mathrm{d} \rho / \mathrm{dt}= & \mathrm{S}_{\mathrm{x}} \operatorname{diag}\left(\mathrm{dp}_{\mathrm{x}, 1} / \mathrm{dt}, \mathrm{dp}_{\mathrm{x}, 0} / \mathrm{dt}, \mathrm{dp}_{\mathrm{x},-1} / \mathrm{dt}\right) \\
& +\mathrm{S}_{\mathrm{y}} \operatorname{diag}\left(\mathrm{dp}_{\mathrm{y}, 1} / \mathrm{dt}, \mathrm{dp}_{\mathrm{y}, 0} / \mathrm{dt}, \mathrm{dp}_{\mathrm{y},-1} / \mathrm{dt}\right)
\end{aligned}
$$

Generalizing to arbitrary L-spin, Eqs. (7.A1) and (7.A2) can be used to generate 2(2L+1) coupled differential equations for the p-coefficients:

$$
\begin{aligned}
& \mathrm{dp}_{\mathrm{y}, \mathrm{m}} / \mathrm{dt}=\omega_{\mathrm{SL}} \mathrm{m} \mathrm{p}_{\mathrm{x}, \mathrm{m}}+\Sigma_{\mathrm{m}}, \mathrm{W}_{\mathrm{m} \mathrm{m}}, \mathrm{p}_{\mathrm{y}, \mathrm{m}}, \\
& \mathrm{dp}_{\mathrm{x}, \mathrm{m}} / \mathrm{dt}=-\omega_{\mathrm{SL}} \mathrm{m} \mathrm{p}_{\mathrm{y}, \mathrm{m}}+\Sigma_{\mathrm{m}}, \mathrm{W}_{\mathrm{m} \mathrm{m}}, \mathrm{p}_{\mathrm{x}, \mathrm{m}^{\prime},}
\end{aligned}
$$

which are equivalent to eq.(59) in ref. [29]. In the absence of relaxation $(\underline{W}=0)$, the solutions are the well-known sinusoidal oscillations, but with relaxation the solutions are much more complicated.

For $\mathrm{L}=1 / 2$, the four coupled Eqs. (7.A3) can be solved analytically, as shown in Appendix B. For $\mathrm{L}>1 / 2$, it is more convenient to evaluate $\Delta \mathrm{p}=\mathrm{dp} / \mathrm{dt} \Delta \mathrm{t}$ numerically timestep by time-step, using

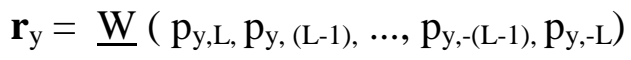

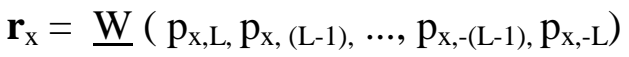




$$
\begin{aligned}
& \Delta \mathrm{p}_{\mathrm{y}, \mathrm{m}}=\left\{\omega_{\mathrm{SL}} \mathrm{m} \mathrm{p}_{\mathrm{x}, \mathrm{m}}+\mathrm{r}_{\mathrm{y}, \mathrm{m}}\right\} \Delta \mathrm{t} \\
& \Delta \mathrm{p}_{\mathrm{x}, \mathrm{m}}=\left\{-\omega_{\mathrm{SL}} \mathrm{m} \mathrm{p}_{\mathrm{y}, \mathrm{m}}+\mathrm{r}_{\mathrm{y}, \mathrm{m}}\right\} \Delta \mathrm{t} \\
& \mathrm{p}_{\mathrm{y}, \mathrm{m}}(\mathrm{t}+\Delta \mathrm{t})=\mathrm{p}_{\mathrm{y}, \mathrm{m}}(\mathrm{t})+\Delta \mathrm{p}_{\mathrm{y}, \mathrm{m}} \\
& \mathrm{p}_{\mathrm{x}, \mathrm{m}}(\mathrm{t}+\Delta \mathrm{t})=\mathrm{p}_{\mathrm{x}, \mathrm{m}}(\mathrm{t})+\Delta \mathrm{p}_{\mathrm{x}, \mathrm{m}}
\end{aligned}
$$

A time step of $\Delta \mathrm{t}=0.1 \mu \mathrm{s}$ was shown to produce the same results as shorter steps and was used in all the numerical simulations. Alternatively, one can use Abragam's exponentialmatrix solutions [29].

\section{Appendix B. Analytical REDOR Signal for $L=1 / 2$.}

For $L=1 / 2$, Eq. (7.A3) gives four coupled equations,

$$
\begin{aligned}
& \mathrm{dp}_{\mathrm{y},+1 / 2} / \mathrm{dt}=\omega_{\mathrm{SL}} 1 / 2 \mathrm{p}_{\mathrm{x},+1 / 2}-\mathrm{r}\left(\mathrm{p}_{\mathrm{y},+1 / 2}-\mathrm{p}_{\mathrm{y},-1 / 2}\right) \\
& \mathrm{dp}_{\mathrm{y},-1 / 2} / \mathrm{dt}=\omega_{\mathrm{SL}}(-1 / 2) \mathrm{p}_{\mathrm{x},-1 / 2}+\mathrm{r}\left(\mathrm{p}_{\mathrm{y},+1 / 2}-\mathrm{p}_{\mathrm{y},-1 / 2}\right) \\
& \mathrm{dp}_{\mathrm{x},+1 / 2} / \mathrm{dt}=-\omega_{\mathrm{SL}} 1 / 2 \mathrm{p}_{\mathrm{y},+1 / 2}-\mathrm{r}\left(\mathrm{p}_{\mathrm{x},+1 / 2}-\mathrm{p}_{\mathrm{x},-1 / 2}\right) \\
& \mathrm{dp}_{\mathrm{x},-1 / 2} / \mathrm{dt}=-\omega_{\mathrm{SL}}\left(-1 / 2 \mathrm{p}_{\mathrm{y},-1 / 2}\right)+\mathrm{r}\left(\mathrm{p}_{\mathrm{x},+1 / 2}-\mathrm{p}_{\mathrm{x},-1 / 2}\right)
\end{aligned}
$$

with $r=1 /\left(2 \mathrm{~T}_{1 \mathrm{~L}}\right)$. Given that $\mathrm{p}_{\mathrm{x},-1 / 2}(0)=\mathrm{p}_{\mathrm{x},+1 / 2}(0)$ and $\mathrm{p}_{\mathrm{y},-1 / 2}(0)=-\mathrm{p}_{\mathrm{y},+1 / 2}(0)$, we can set $\mathrm{p}_{\mathrm{x},-1 / 2}$ $=p_{x,+1 / 2}=p_{x}$ and $p_{y,-1 / 2}=-p_{y,+1 / 2}=-p_{y}$. This makes Eqs. $(\mathrm{B} 1 \mathrm{~b})$ and $(\mathrm{B} 1 \mathrm{~d})$ redundant, leaving

$$
\begin{aligned}
& \mathrm{dp}_{\mathrm{y}} / \mathrm{dt}=\mathrm{p}_{\mathrm{x}} \omega_{\mathrm{SL}} / 2-\mathrm{p}_{\mathrm{y}} 2 \mathrm{r} \\
& \mathrm{dp}_{\mathrm{x}} / \mathrm{dt}=\mathrm{p}_{\mathrm{y}}\left(-\omega_{\mathrm{SL}}\right) / 2
\end{aligned}
$$

Here, it is straightforward to incorporate transverse $T_{2 S}$ relaxation of the $S$ spin:

$$
\begin{aligned}
& \mathrm{dp}_{\mathrm{y}} / \mathrm{dt}=\mathrm{p}_{\mathrm{x}} \omega_{\mathrm{SL}} / 2-\mathrm{p}_{\mathrm{y}}\left(1 / \mathrm{T}_{2 \mathrm{~S}}+1 / \mathrm{T}_{1 \mathrm{~L}}\right) \\
& \mathrm{dp}_{\mathrm{x}} / \mathrm{dt}=\mathrm{p}_{\mathrm{y}}\left(-\omega_{\mathrm{SL}}\right) / 2-\mathrm{p}_{\mathrm{x}} / \mathrm{T}_{2 \mathrm{~S}}
\end{aligned}
$$


The factor of $1 / 2$ in front of $\omega_{\mathrm{SL}}$ is usually avoided in calculations limited to spin- $1 / 2$ by writing a factor of 2 in front of the right-hand side of the Hamiltonian in Eq. (7.7), but this is not advisable in calculations for general $L$ that are also of interest here. The solutions for the initial condition $\mathrm{p}_{\mathrm{x}}=1, \mathrm{p}_{\mathrm{y}}=0$ differ qualitatively whether

$$
w=\sqrt{\omega_{S L}^{2} T_{1 L}^{2}-1}
$$

is real or imaginary:

For long $\mathrm{T}_{1 \mathrm{~L}}>1 / \omega_{\mathrm{SL}}, w$ is real, and we obtain oscillatory solutions

$$
\begin{aligned}
& p_{x}\left(t, T_{1 L}\right)=e^{-\left(\frac{1}{2 T_{1 L}}+\frac{1}{T_{2 S}}\right) t}\left(\cos \frac{w t}{2 T_{1 L}}+\frac{1}{w} \sin \frac{w t}{2 T_{1 L}}\right), \\
& p_{y}\left(t, T_{1 L}\right)=\frac{1}{w} e^{-\left(\frac{1}{2 T_{1 L}}+\frac{1}{T_{2 S}}\right) t}\left(\sin \frac{w t}{2 T_{1 L}}\right) T_{S L} \omega_{S L}
\end{aligned}
$$

For short $\mathrm{T}_{1 \mathrm{~L}}<1 / \omega_{\mathrm{SL}}, w$ is imaginary, and we obtain

$$
\begin{aligned}
& p_{x}\left(t, T_{1 L}\right)=e^{-\left(\frac{1}{2 T_{L L}}+\frac{1}{T_{2 S}}\right) t}\left(\cosh \frac{|w| t}{2 T_{1 L}}+\frac{1}{|w|} \sinh \frac{|w| t}{2 T_{1 L}}\right), \\
& p_{y}\left(t, T_{1 L}\right)=\frac{1}{|w|} e^{-\left(\frac{1}{2 T_{1 L}}+\frac{1}{T_{2 S}}\right) t}\left(\sinh \frac{|w| t}{2 T_{1 L}}\right) T_{S L} \omega_{S L}
\end{aligned}
$$

Note that in recoupling experiments, $\mathrm{t}$ is usually denoted as $\mathrm{Nt}_{\mathrm{r}}$. Curves calculated based on Eqs. (7.B5a) and (7.B5c) are shown in Fig. 7.2(a).

The reference signal $\mathrm{S}_{0}$ is obtained by applying one more or one less inversion pulse at the center of the recoupling period. This is equivalent to an inversion of the effective heteronuclear coupling frequency $\omega_{\mathrm{sL}}$ in Eq.(7.B2) at $\mathrm{Nt}_{\mathrm{r}} / 2$. The solutions in Appendix B can be used to calculate the initial condition for the second part of the heteronuclear evolution, i.e.

$\mathrm{p}_{\mathrm{x}}{ }^{0}(0)=\mathrm{p}_{\mathrm{x}}\left(\mathrm{Nt}_{\mathrm{r}} / 2\right)$ and $\mathrm{p}_{\mathrm{y}}{ }^{0}(0)=\mathrm{p}_{\mathrm{y}}\left(\mathrm{Nt}_{\mathrm{r}} / 2\right)$ with $\mathrm{p}_{\mathrm{x}}$ and $\mathrm{p}_{\mathrm{y}}$ as given in Eq. (7.B5).

For long $\mathrm{T}_{1 \mathrm{~L}}>1 / \omega_{\mathrm{SL}}, w$ is real, and 


$$
p_{x}^{0}\left(t, T_{1 L}\right)=e^{-\left(\frac{1}{2 T_{1 L}}+\frac{1}{T_{2 S}}\right) t}\left(-\frac{1}{w^{2}} \cos \frac{w t}{2 T_{1 L}}+\frac{1}{w} \sin \frac{w t}{2 T_{1 L}}-\frac{\omega_{S L}^{2} T_{1 L}^{2}}{w^{2}}\right)
$$

For short $\mathrm{T}_{1 \mathrm{~L}}<1 / \omega_{\mathrm{SL}}, w$ is imaginary, and

$$
p_{x}^{0}\left(t, T_{1 L}\right)=e^{-\left(\frac{1}{2 T_{1 L}}+\frac{1}{T_{2 S}}\right) t}\left(-\frac{1}{w^{2}} \cosh \frac{|w| t}{2 T_{1 L}}+\frac{1}{|w|} \sinh \frac{|w| t}{2 T_{1 L}}-\frac{\omega_{S L}^{2} T_{1 L}^{2}}{w^{2}}\right)
$$

For all $\mathrm{T}_{1 \mathrm{~L}}$ values,

$$
p_{y}^{0}\left(t, T_{1 Q}\right)=\left(1-\frac{1}{w^{2}}\right) e^{-\left(\frac{1}{2 T_{1 L}}+\frac{1}{T_{2 S}}\right) t}\left(1-e^{-\frac{w t}{2 T_{1 L}}}\right)
$$

Curves calculated from Eqs. (7.B6a) and (7.B6b) are shown in Fig. 7. 2(b).

\section{Appendix C. $\mathbf{T}_{1 \mathrm{Q}}-$ Dependence of the REDOR decay constant $\mathbf{T}_{\mathrm{SL}}$}

From numerical REDOR simulation for spin $\mathrm{L}=1$ with $\mathrm{T}_{1 \mathrm{Q}}$ relaxation of the $\mathrm{L}-\mathrm{spin}$, we have determined the dependence of the time constant $\mathrm{T}_{\mathrm{SL}}$ in REDOR $\mathrm{S}$ and $\mathrm{S}_{0}$ experiments on $\mathrm{T}_{1 \mathrm{Q}}$, see Fig. 7. 4. Some aspects of these $\mathrm{T}_{\mathrm{SL}} \mathrm{vs}$. $\mathrm{T}_{1 \mathrm{Q}}$ curves were difficult to explain quantitatively based on a qualitative analysis. In the following, we derive these features based on the analytical analysis for $L=1 / 2$. For all cases, we assume that $T_{2 S} \gg$ $\mathrm{T}_{1 \mathrm{~L}}$, so that the effect of $\mathrm{T}_{1 \mathrm{~L}}$ is not masked by fast $\mathrm{T}_{2 \mathrm{~S}}$ decay. Analytical simulations of $\mathrm{T}_{\mathrm{SL}}$ vs. $\mathrm{T}_{1 \mathrm{~L}}$ for spin $\mathrm{L}=1 / 2$ are plotted in Fig. 7. S2 (see Supporting Material); they closely resemble the numerical results for $\mathrm{L}=1$ shown in Fig. 7.4.

For short $\mathrm{T}_{1 \mathrm{~L}}<<1 / \omega_{\mathrm{SL}},|w| \sim 1$, and Eq. (7.B5c) for the dephased signal $\mathrm{S}$ can be approximated as

$$
p_{x}\left(t, T_{1 L}\right) \approx e^{-\left(\frac{1-|w|}{2 T_{1 L}}\right) t}
$$

so the decay constant is

$$
T_{S L}=\frac{2 T_{1 L}}{1-|w|} \approx \frac{2 T_{1 L}}{\frac{1}{2}\left(2 T_{1 L}\right)^{2}\left(\frac{\omega_{S L}}{2}\right)^{2}}=\frac{4}{\omega_{S L}^{2} T_{1 L}}
$$

which gives Eq.(7.11).

For long $\mathrm{T}_{1 \mathrm{~L}} \gg 1 / \omega_{\mathrm{SL}}$, we have $w \sim \mathrm{T}_{1 \mathrm{~L}} \omega_{\mathrm{SL}}$ and Eq. (7.B5a) can be simplified to 


$$
p_{x}\left(t, T_{1 L}\right) \approx e^{-\frac{t}{2 T_{1 L}}}\left(\cos \frac{\omega_{S L} t}{2}\right)
$$

where the decay is due to the $\cos \left(\omega_{\mathrm{SL}} \mathrm{t} / 2\right)$ oscillation and therefore independent of $\mathrm{T}_{1 \mathrm{~L}}$; this corresponds to the long-time plateaus in the $\mathrm{T}_{\mathrm{SL}} \mathrm{vs}$. $\mathrm{T}_{1 \mathrm{~L}}$ curves for REDOR $\mathrm{S}$ in Fig. 7. S2.

The REDOR reference signal $\mathrm{S}_{0}$ of Eq. (7.B6b) can be simplified for short $\mathrm{T}_{1 \mathrm{~L}}<<$ $1 / \omega_{\mathrm{SL}}$, where $|w| \sim 1$ :

$$
\left.p_{x}^{0}\left(t, T_{1 L}\right)=e^{-\frac{1}{2 T_{1 L}} t}\left(\cosh \frac{|w| t}{2 T_{1 L}}+\sinh \frac{|w| t}{2 T_{1 L}}\right)=e^{-\frac{1}{2 T_{1 L}} t} e^{\frac{|w|}{2 T_{1 L}} t}=e^{-\frac{(1-|w|)}{2 T_{1 L}} t} \approx p_{x}\left(t, T_{1 L}\right) \text { (7.C } 3 \mathrm{a}\right)
$$

In other words, $\mathrm{S}_{0} \approx \mathrm{S}$, which explains the overlap of the curves for $\mathrm{S}$ and $\mathrm{S}_{0}$ in Fig. 7.S2. for short $\mathrm{T}_{1 \mathrm{Q}}$ values.

For long $\mathrm{T}_{1 \mathrm{~L}} \gg 1 / \omega_{\mathrm{SL}}, w \sim \mathrm{T}_{1 \mathrm{~L}} \omega_{\mathrm{SL}}$, and

$$
p_{x}^{0}\left(t, T_{1 L}\right)=e^{-\frac{t}{2 T_{1 L}}}(0+0-(-1))=e^{-\frac{t}{2 T_{1 L}}}
$$

which gives $\mathrm{T}_{\mathrm{SL}} \sim 2 \mathrm{~T}_{1 \mathrm{~L}}$ independent of $\omega_{\mathrm{SL}}$, as observed in Fig. 7.S2.

\section{Appendix D. Supplementary data}

Supplementary data associated with this article can be found, in the online version, at doi:10.1016/j.jmr.2008.12.021.

\section{References}

[1] T. Gullion, A.J. Vega, Measuring heteronuclear dipolar couplings for $\mathrm{I}=1 / 2, \mathrm{~S}>1 / 2$ spin pairs by REDOR and REAPDOR NMR. Progr. Nucl. Magn. Reson. Spectros. 47 (2005) 123-136.

[2] I. Schnell, Dipolar recoupling in fast-MAS solid-state NMR spectroscopy. Progr. Nucl. Magn. Reson. Spectros. 45 (2004) 145-207.

[3] T. Gullion, Detecting 13C-17O dipolar interactions by rotational-echo, adiabaticpassage, double-resonance NMR. J. Magn. Reson. 117 (1995) 326-329. 
[4] E. Hughes, T. Gullion, A. Goldbourt, S. Vega, A.J. Vega, Internuclear distance determination of $\mathrm{S}=1, \mathrm{I}=1 / 2$ spin pairs using REAPDOR NMR. J. Magn. Reson. 156 (2002) 230-241.

[5] K. Schmidt-Rohr, J.D. Mao, Selective observation of nitrogen-bonded carbons in solidstate NMR by saturation-pulse induced dipolar exchange with recoupling. Chem Phys Lett 359 (2002) 403-441.

[6] S. Cavadini, A. Abraham, G. Bodenhausen, Coherence transfer between spy nuclei and nitrogen-14 in solids. J. Magn. Reson. 190 (2008) 160-164.

[7] Z. Gan, 13C/14N heteronuclear multiple-quantum correlation with rotary resonance and REDOR dipolar recoupling. J. Magn. Reson. 184 (2007) 39-43.

[8] A. Abragam, Principles of Nuclear Magnetism, Ch. XI, Sect. III, Clarendon Press, Oxford, 1983.

[9] C.-G. Hoelger, E. Roessler, B. Wehrle, F. Aguilar-Parrilla, H.-H. Limbach, SelfDecoupling of 15N-14N Dipole-Quadrupole Couplings in 15N CPMAS NMR Spectra and Molecular Motions in Crystalline Hydrazine Sulfate, p-(Diethylamino)benzaldehyde Diphenylhydrazone, and Its Solid Solution in Polycarbonate. J. Phys. Chem. 99 (1995) 14271-14276.

[10] R.W. Schurko, R.E. Wasylishen, J.H. Nelson, Effect of cobalt-59 self-decoupling on the solid-state 31P CP/MAS NMR spectra of cobaloximes. J. Phys. Chem. 100 (1996) 80578060 .

[11] R.K. Harris, A.C. Olivieri, Quadrupolar effects transferred to spin-1/2 magic-angle spinning spectra of solids. Progr. Nucl. Magn. Reson. Spectros. 24 (1992) 435-456.

[12] A. Naito, P.B. Barker, C.A. McDowell, Two-dimensional nuclear magnetic resonance studies on a single crystal of 1-alanine. Separation of the local dipolar fields; and 2D exchange spectroscopy of the nitrogen-14 relaxation processes. J. Chem. Phys. 81 (1984) 1583-1591.

[13] K. Saalwachter, K. Schmidt-Rohr, Relaxation-Induced Dipolar Exchange with Recoupling-An MAS NMR Method for Determining Heteronuclear Distances without Irradiating the Second Spin. J. Magn. Reson. 145 (2000) 161-172. 
[14] A. Suter, M. Mali, J. Roos, D. Brinkmann, Mixed magnetic and quadrupolar relaxation in the presence of a dominant static Zeeman Hamiltonian. J. Phys.: Condens. Matter 10 (1998) 5977-5994.

[15] J.P. Yesinowski, Magnetization-recovery experiments for static and MAS-NMR of I = 3/2 nuclei. J. Magn. Reson. 180 (2006) 147-161.

[16] H.W. Spiess, Deuteron spin alignment: a probe for studying ultraslow motions in solids and solid polymers. J. Chem. Phys. 72 (1980) 6755-6762.

[17] A.E. McDermott, F. Creuzet, R. Gebhard, K. van der Hoef, M.H. Levitt, J. Herzfeld, J. Lugtenburg, R.G. Griffin, Determination of internuclear distances and the orientation of functional groups by solid-state NMR: Rotational resonance study of the conformation of retinal in bacteriorhodopsin. Biochemistry 33 (1994) 6129-6136.

[18] M. Ernst, A. Verhoeven, B.H. Meier, High-speed magic-angle spinning 13C MAS NMR spectra of adamantane: self-decoupling of the heteronuclear scalar interaction and proton spin diffusion. J. Magn. Reson. 130 (1998) 176-185.

[19] B.H. Meier, Polarization transfer and spin diffusion in solid-state NMR. Adv Magn Opt Reson 18 (1994) 1-116.

[20] M. Bak, J.T. Rasmussen, N.C. Nielsen, SIMPSON. A general simulation program for solid-state NMR spectroscopy. J. Magn. Reson. 147 (2000) 296-330.

[21] A.J. Shaka, J. Keeler, M.B. Smith, R. Freeman, Spatial localization of NMR signals in an inhomogeneous radiofrequency field. J. Magn. Reson. 61 (1985) 175-180.

[22] K. Schmidt-Rohr, H.W. Spiess, Multidimensional Solid-State NMR and Polymers, 1994.

[23] S.K. Sarkar, P.E. Young, C.E. Sullivan, D.A. Torchia, Proline ring motion in solid state: a deuterium NMR study. Pept.: Struct. Funct., Proc. Am. Pept. Symp., 9th (1985) 121124.

[24] H.W. Spiess, Rotation of molecules and nuclear spin relaxation. Nucl. Magn. Reson. 15 (1978) 55.

[25] S.F. Liu, J.D. Mao, K. Schmidt-Rohr, A Robust Technique for Two-Dimensional Separation of Undistorted Chemical-Shift Anisotropy Powder Patterns in Magic-AngleSpinning NMR. J. Magn. Reson. 155 (2002) 15-28. 
[26] Z. Gu, D.G. Drueckhammer, L. Kurz, K. Liu, D.P. Martin, A. McDermott, Solid State NMR Studies of Hydrogen Bonding in a Citrate Synthase Inhibitor Complex.

Biochemistry 38 (1999) 8022-8031.

[27] S.H. Alarcon, A.C. Olivieri, P. Jonsen, Carbon-13 CPMAS NMR study of solid arylazonaphthols. Evidence of carbon-13, nitrogen-14 self-decoupling induced by a solid-state proton transfer reaction. Perkin Trans 2 (1993) 1783-1786.

[28] A. Nayeem, J.P. Yesinowski, Calculation of magic-angle spinning nuclear magnetic resonance spectra of paramagnetic solids. J. Chem. Phys. 89 (1988) 4600-4608.

[29] A. Abragam, Principles of Nuclear Magnetism, Ch. X, Sect. IV, Clarendon Press, Oxford, 1983.

\section{Tables and Figures}

Table 7.1. Table of normalized SPIDER $S, S_{0}, S_{00}, \Delta S=S_{0}-S, \Delta S_{0}=S_{00}-S_{0}$, and $\Delta S_{\text {tot }}=$ $\mathrm{S}_{00^{-}} \mathrm{S}$ intensities for long $\left(\mathrm{T}_{1 \mathrm{Q}}>100 / \delta\right)$, intermediate $\left(10 / \delta<\mathrm{T}_{1 \mathrm{Q}}<100 / \delta\right)$, short $\left(1 / \delta<\mathrm{T}_{1 \mathrm{Q}}<\right.$ $10 / \delta)$, very short $\left(\mathrm{T}_{1 \mathrm{Q}} \sim 1 / \delta\right)$, and extremely short $\left(\mathrm{T}_{1 \mathrm{Q}}<<1 / \delta\right)$ quadrupolar relaxation times, for $\mathrm{L}=1$ and $\mathrm{Nt}_{\mathrm{r}}=20 / \delta$. Note that the definition of the S-L dipolar coupling constant $\delta$ includes the factor $2 \pi$.

\begin{tabular}{|l|c|c|c|c|c|c|}
\hline \multicolumn{1}{|c|}{ Signal } & $\mathrm{S}_{00}$ & $\mathrm{~S}_{0}$ & $\mathrm{~S}$ & $\Delta \mathrm{S}$ & $\Delta \mathrm{S}_{0}$ & $\Delta \mathrm{S}_{\text {tot }}$ \\
\hline $\begin{array}{l}\text { Long } \\
\left(\mathrm{T}_{1 \mathrm{Q}}>100 / \delta\right)\end{array}$ & 1 & 1 & 0.33 & 0.67 & 0 & 0.67 \\
\hline $\begin{array}{l}\text { Intermediate } \\
\left(10 / \delta<\mathrm{T}_{1 \mathrm{Q}}<100 / \delta\right)\end{array}$ & $\sim 0.9$ & $\sim 0.8$ & $\sim 0.4$ & $\sim 0.4$ & $\sim 0.1$ & $\sim 0.5$ \\
\hline $\begin{array}{l}\text { Short } \\
\left(1 / \delta<\mathrm{T}_{1 \mathrm{Q}}<10 / \delta\right)\end{array}$ & $\sim 0.8$ & $\sim 0.5$ & $\sim 0.5$ & $\sim 0$ & $\sim 0.3$ & $\sim 0.3$ \\
\hline $\begin{array}{l}\text { Very short } \\
\left(\mathrm{T}_{1 \mathrm{Q}} \sim 1 / \delta\right)\end{array}$ & $\sim 0.8$ & $\sim 0.7$ & $\sim 0.7$ & 0 & $\sim 0.1$ & $\sim 0.1$ \\
\hline $\begin{array}{l}\text { Extremely short } \\
\left(\mathrm{T}_{1 \mathrm{Q}}<1 / \delta\right)\end{array}$ & $\sim 1$ & $\sim 1$ & $\sim 1$ & 0 & 0 & 0 \\
\hline
\end{tabular}


Table 7.2. Summary of ${ }^{13} \mathrm{C}$ chemical shift principal values obtained from SUPER spectra of Boc-Arg-OH (compound 1) and Boc- $\mathrm{Arg}(\mathrm{Z})_{2}-\mathrm{OH}$ (compound 2), and isotropic chemical shifts from MAS. Principal values have error margins of $\pm 5 \mathrm{ppm}$.

\begin{tabular}{|l|l|l|l|l|l|l|}
\hline Compound & Carbon site & \multicolumn{3}{|l|}{ Chemical shift components (ppm) } & $\Delta \sigma$ & $\sigma_{\text {iso }}$ \\
\hline 1 & COO $^{-}$ & 242 & 174 & 124 & 118 & 180 \\
\hline $\mathbf{2}$ & COOH & $\mathbf{2 5 7}$ & $\mathbf{1 5 3}$ & $\mathbf{1 1 3}$ & $\mathbf{1 4 4}$ & $\mathbf{1 7 4}$ \\
\hline 1 & NC=O & 245 & 130 & 112 & 133 & 162 \\
\hline $\mathbf{2}$ & NC=O & $\mathbf{2 3 2}$ & $\mathbf{1 2 5}$ & $\mathbf{1 2 5}$ & $\mathbf{1 0 7}$ & $\mathbf{1 5 9}$ \\
\hline $\mathbf{2}$ & NHCOO & $\mathbf{2 3 4}$ & $\mathbf{1 1 5}$ & $\mathbf{1 1 5}$ & $\mathbf{1 1 9}$ & $\mathbf{1 5 4}$ \\
\hline $\mathbf{2}$ & NCOO & $\mathbf{2 4 2}$ & $\mathbf{1 2 7}$ & $\mathbf{1 2 3}$ & $\mathbf{1 1 9}$ & $\mathbf{1 6 4}$ \\
\hline
\end{tabular}



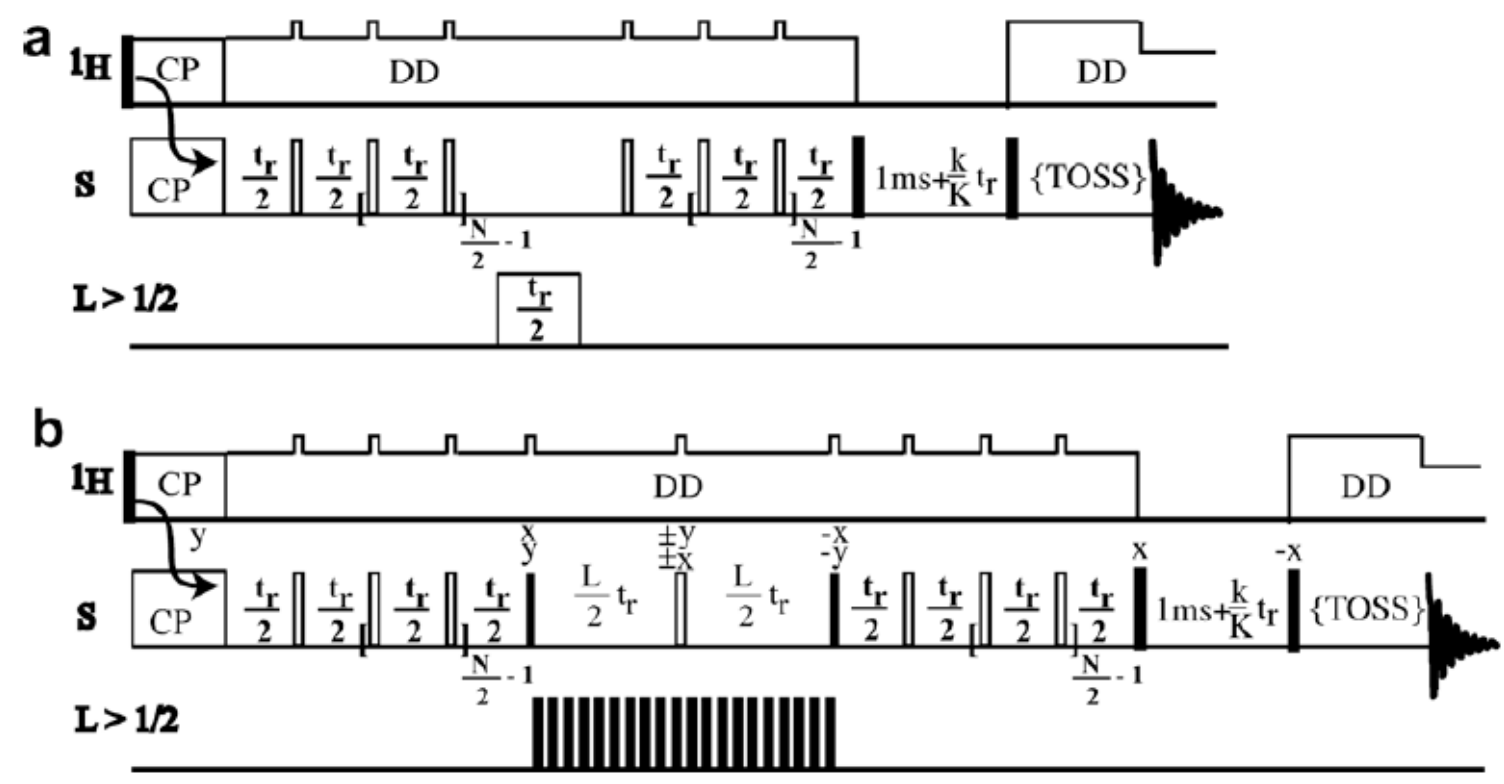

Fig. 7.1. Pulse sequences for (a) $S\{L\}$ REAPDOR and (b) $S\{L\}$ SPIDER with $L>1 / 2$. Pulses with $90^{\circ}$ flip angles are shown as narrow filled rectangles, $180^{\circ}$ pulses as narrow unfilled rectangles. The irradiation on the L-spin in SPIDER consists of closely spaced $\sim 2-\mu \mathrm{s}$ pulses with $\sim 1-\mu$ s spacing. Reference signals $S_{0}$ are obtained without irradiation on the Lspin, but with the train of $180^{\circ}$ pulses on $\mathrm{S}$. 


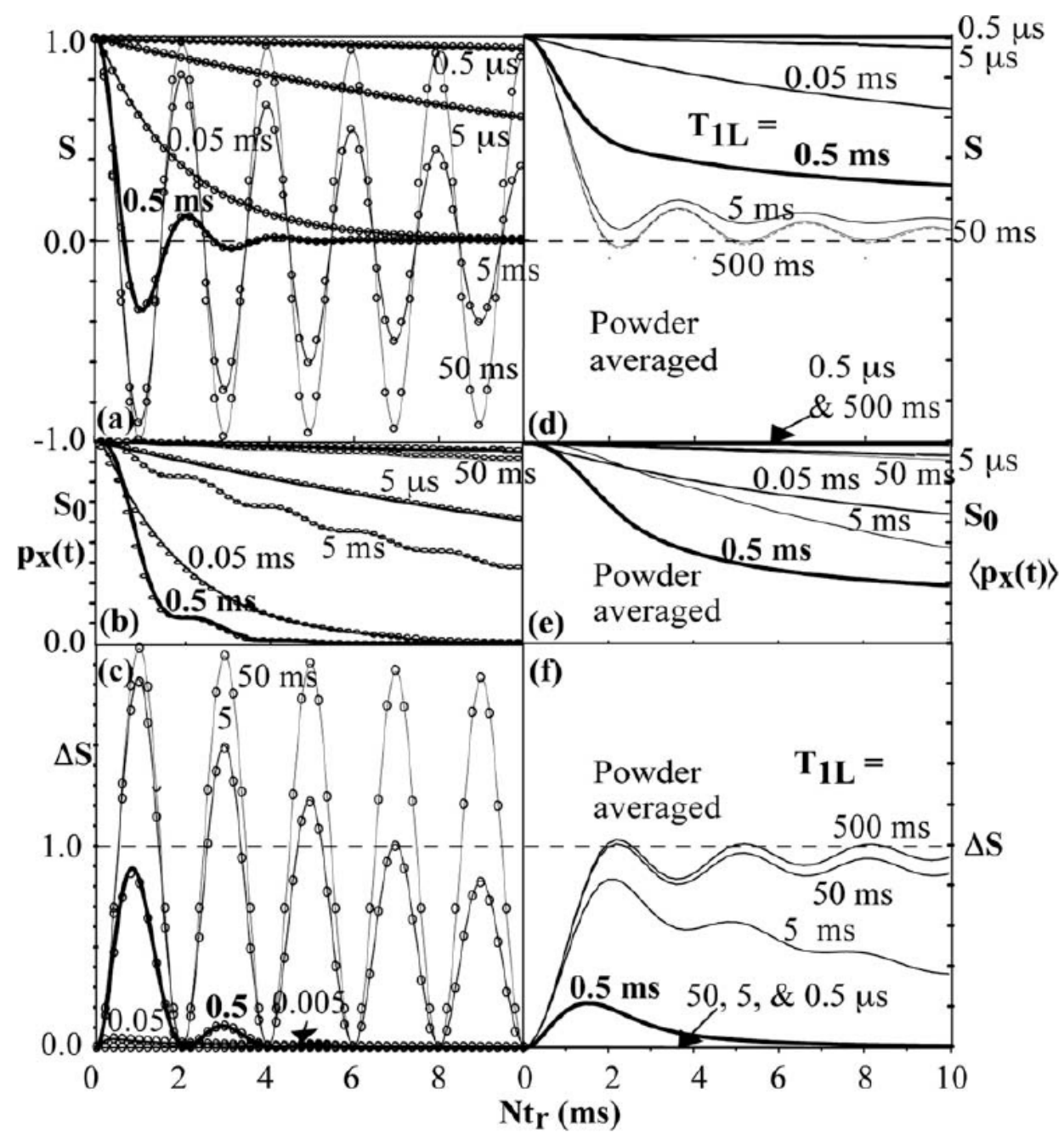

Fig. 7.2. $S\{L\}$ REDOR $S, S_{0}$ and $\Delta S$ simulations for spin $L=1 / 2$ with $T_{1 L}$ relaxation. Singlefrequency analytical simulations are shown in (a-c) with solid lines, numerical simulations are represented by open circles. $\omega_{\mathrm{SL}}=2 \pi 1 \mathrm{kHz}$ was used in both simulations. Numerical powder-averaged REDOR simulations are shown in (d-f) with $\delta_{\mathrm{SL}}=2 \pi 1.5 \mathrm{kHz}$. $\mathrm{T}_{1 \mathrm{~L}}$ values in ms are indicated at each curve. 


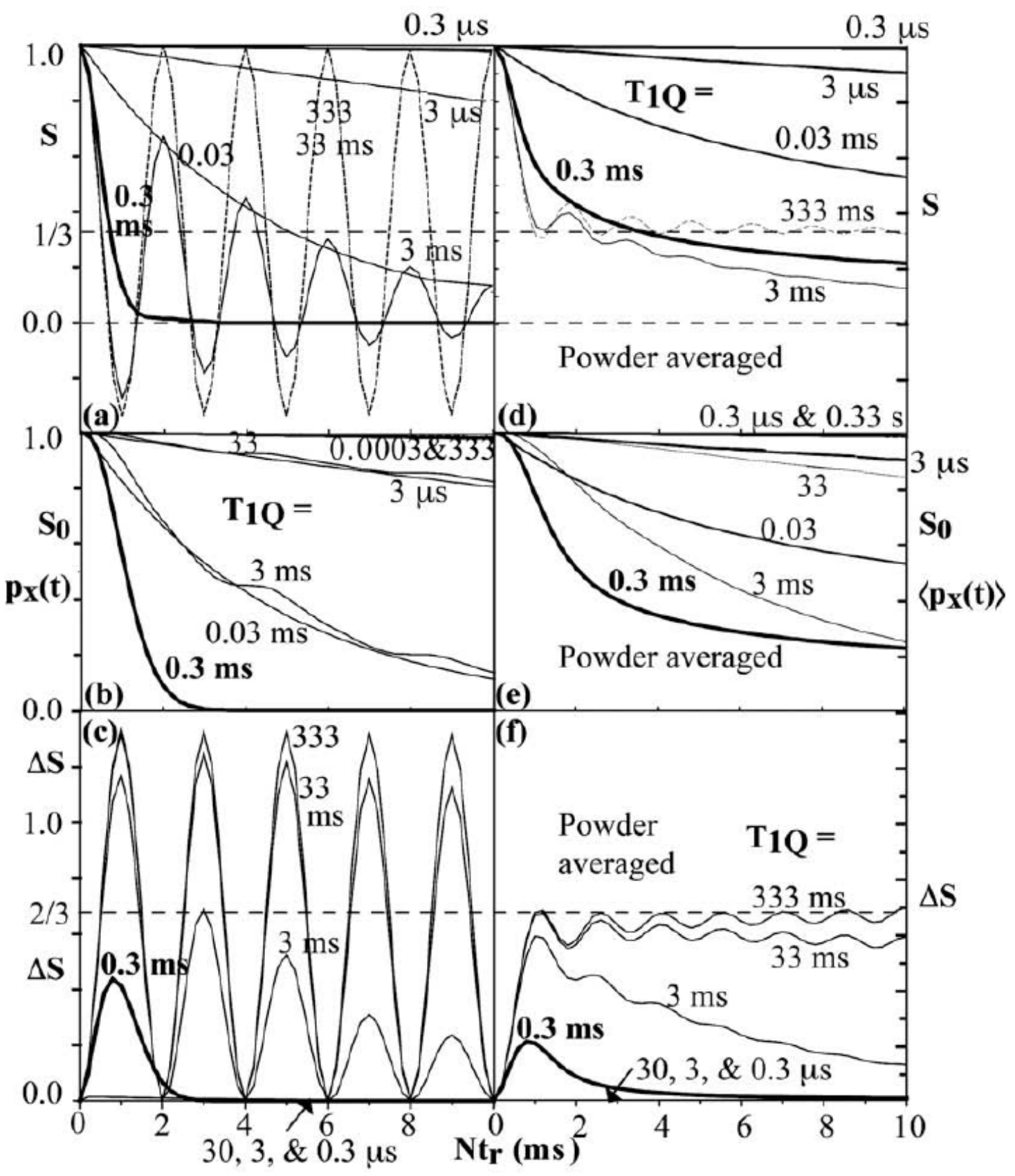

Fig. 7.3. $S\{L\}$ REDOR simulations for spin $L=1$ with $T_{1 Q}$ relaxation. Single-frequency REDOR (a) $\mathrm{S}$, (b) $\mathrm{S}_{0}$, and (c) $\Delta \mathrm{S}$ simulations with $\omega_{\mathrm{SL}}=2 \pi 0.5 \mathrm{kHz}$. Powder-averaged REDOR (d) $\mathrm{S}$, (e) $\mathrm{S}_{0}$, and (f) $\Delta \mathrm{S}$ simulations with $\delta_{\mathrm{SL}}=2 \pi 1.5 \mathrm{kHz}$. 


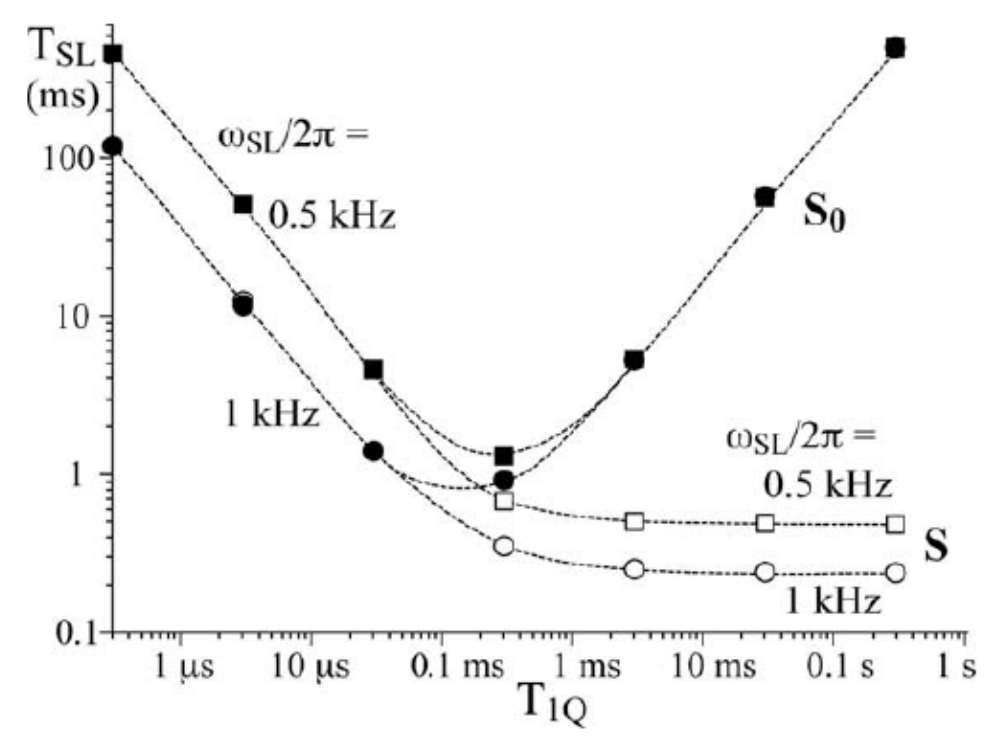

Fig. 7.4. Decay time constants $T_{S L}$ of $R E D O R S$ and $S_{0}$ for spin $L=1$ as a function of $T_{1 Q}$, with two different coupling strengths, $\omega_{\mathrm{SL}}=2 \pi 0.5 \mathrm{kHz}$ and $\omega_{\mathrm{SL}}=2 \pi 1 \mathrm{kHz}$.
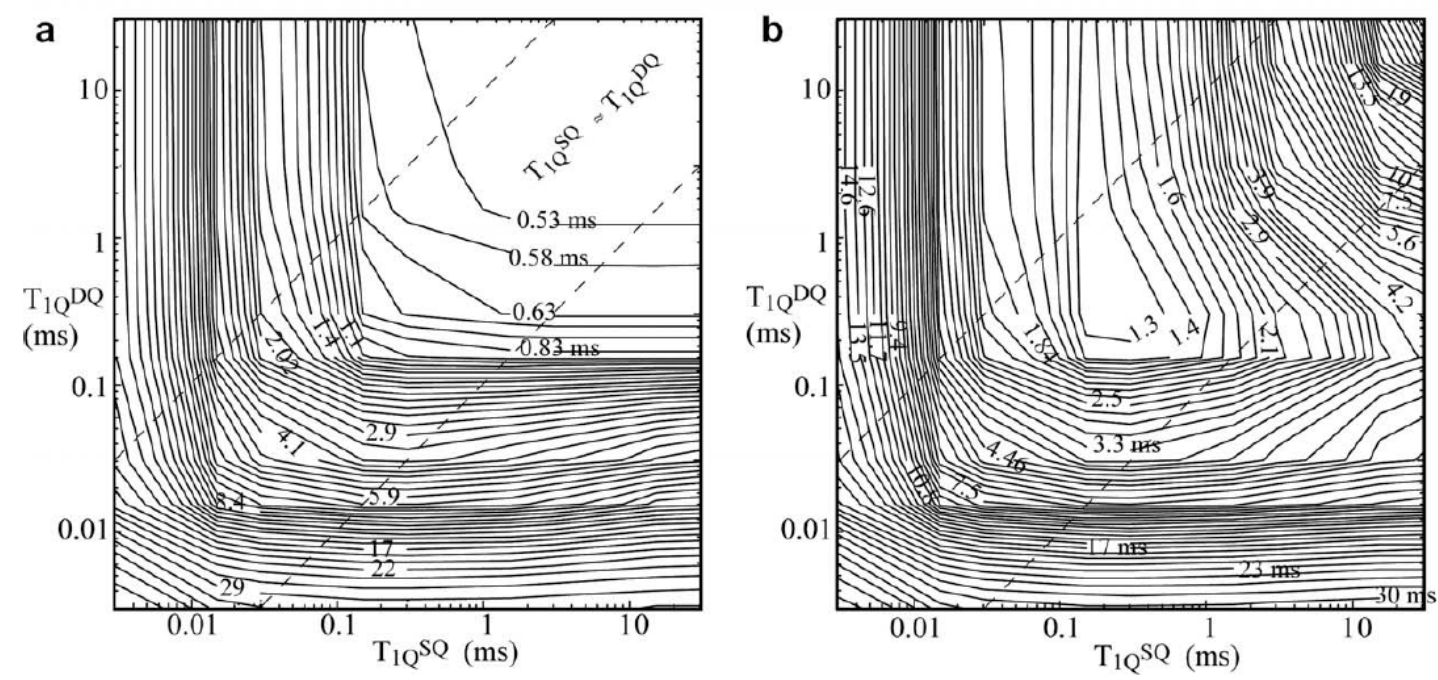

Fig. 7.5. Contour plots of (a) $T_{S L}$ for REDOR $S$ decay and (b) $T_{S L}{ }^{0}$ for REDOR $S_{0}$ decay as a function of $\mathrm{T}_{1 \mathrm{Q}}{ }^{\mathrm{SQ}}$ and $\mathrm{T}_{1 \mathrm{Q}}{ }^{\mathrm{DQ}}$ on a logarithmic scale, for $\mathrm{L}=1$. The coupling strength is $\omega_{\mathrm{SL}}=$ $2 \pi 0.5 \mathrm{kHz}$. 


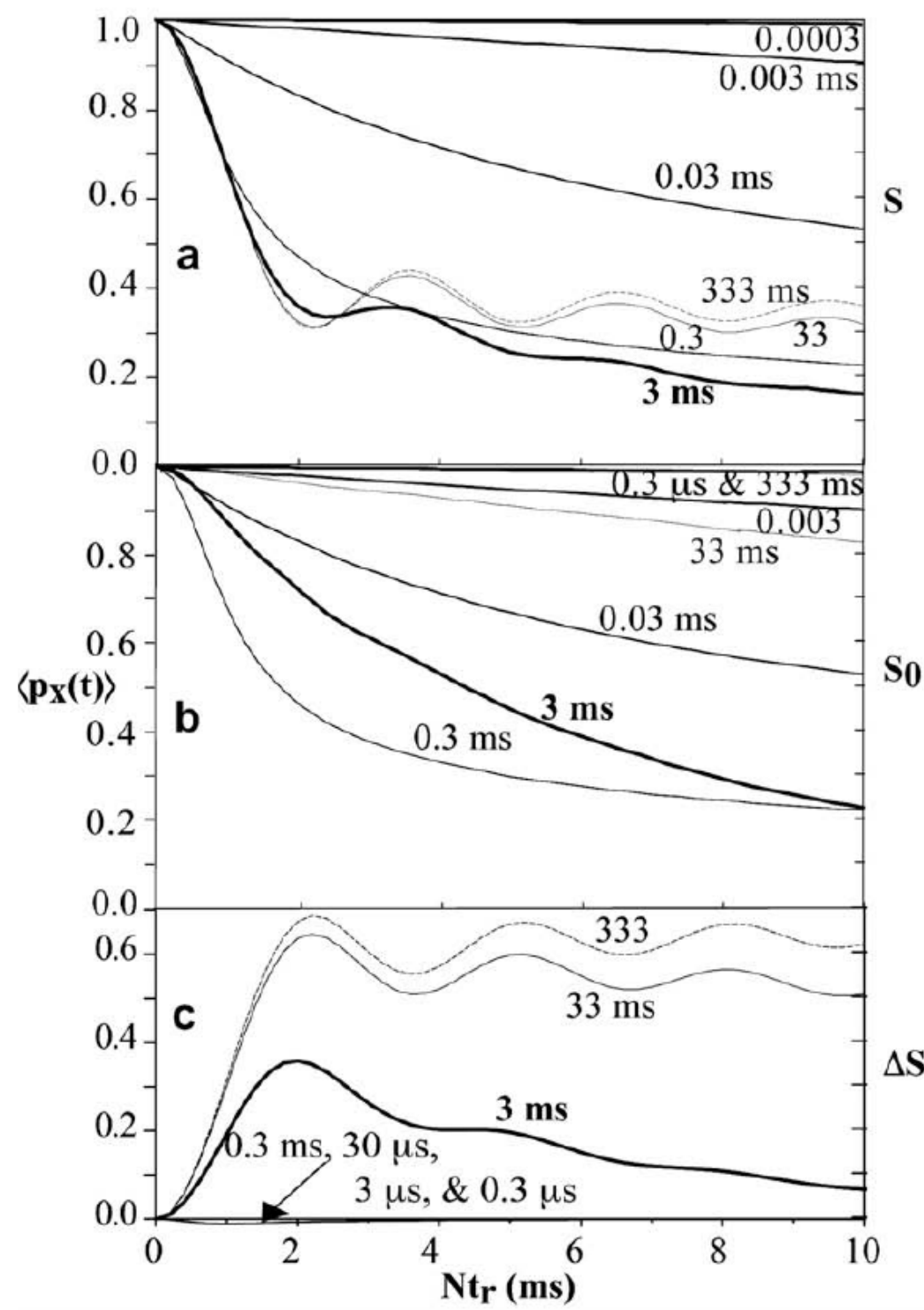

Fig. 7.6. $S\{L\}$ SPIDER simulations for $L=1$ with $T_{1 Q}$ relaxation and powder averaging, for a coupling strength $\delta_{\mathrm{SL}}=2 \pi 1.5 \mathrm{kHz}$, and with 0.8 -ms irradiation on the L-spin. (a) S, (b) $\mathrm{S}_{0}$ and (c) $\Delta \mathrm{S}$. 


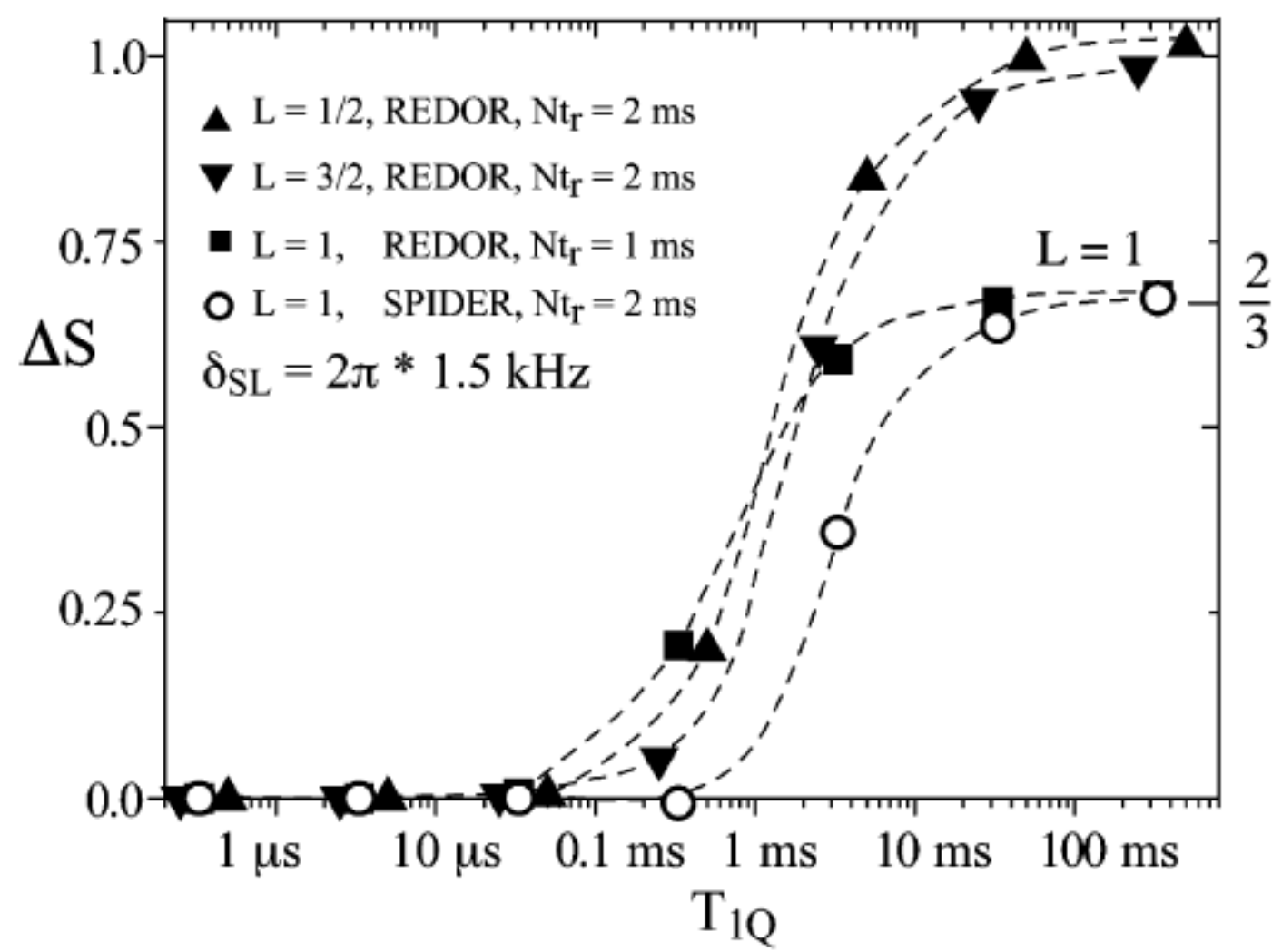

Fig. 7.7. $\mathrm{T}_{1 \mathrm{Q}}$ dependence of $\Delta \mathrm{S}$ intensities extracted from REDOR and SPIDER simulations with $\delta_{\mathrm{SL}}=2 \pi 1.5 \mathrm{kHz}$. Filled triangles: $\mathrm{S}\{\mathrm{L}\}$ REDOR $\Delta \mathrm{S}$ for spin $\mathrm{L}=1 / 2$, at recoupling time of $\mathrm{Nt}_{\mathrm{r}}=2 \mathrm{~ms}$. Filled inverted triangles: $\mathrm{S}\{\mathrm{L}\}$ REDOR $\Delta \mathrm{S}$ for spin $\mathrm{L}=3 / 2$, at $\mathrm{Nt}_{\mathrm{r}}=2 \mathrm{~ms}$. Filled squares: $\mathrm{S}\{\mathrm{L}\}$ REDOR $\Delta \mathrm{S}$ for spin $\mathrm{L}=1$, at $\mathrm{Nt}_{\mathrm{r}}=1 \mathrm{~ms}$. Open circles: $\mathrm{S}\{\mathrm{L}\}$ SPIDER $\Delta \mathrm{S}$ for $\operatorname{spin} \mathrm{L}=1$, at $\mathrm{Nt}_{\mathrm{r}}=2 \mathrm{~ms}$. 

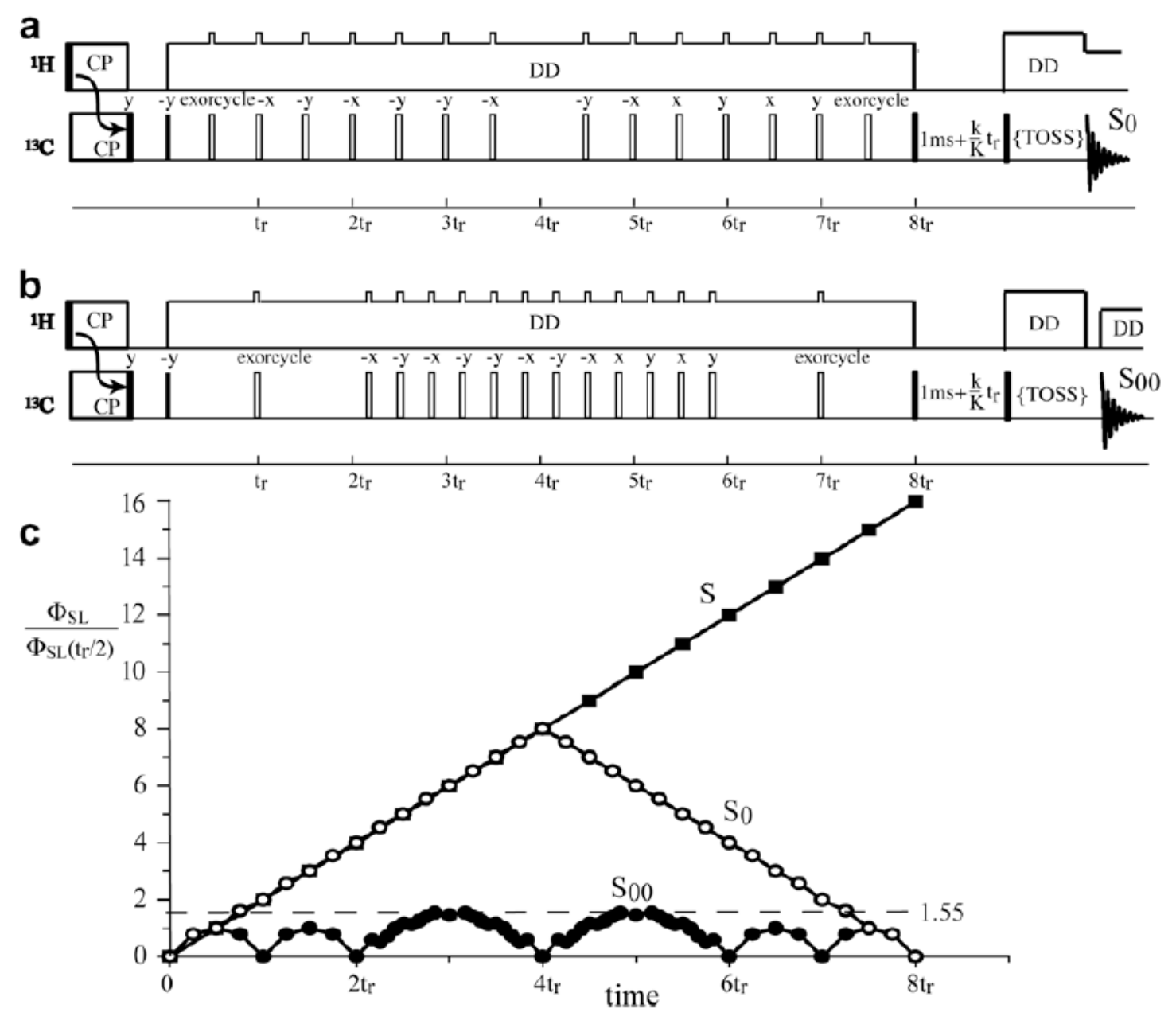

Fig. 7.8. (a) Simplified pulse sequence for measuring $S_{0}$ without irradiation on the quadrupolar nucleus. (b) Matching pulse sequence for measuring an improved reference signal $S_{00}$ with minimal transient S-L recoupling. (c) Simulated evolutions of the phase $\Phi_{\mathrm{SL}}$ of the magnetization in REAPDOR or SPIDER S dephasing (S), REAPDOR or SPIDER reference experiments $\left(S_{0}\right)$ and for the new pulse sequence shown in $(b)\left(S_{00}\right)$. The unit used is the phase $\Phi_{\mathrm{SL}}\left(\mathrm{t}_{\mathrm{r}} / 2\right)$ generated within half a rotation period $\left(\mathrm{t}_{\mathrm{r}} / 2\right)$ under free evolution. 

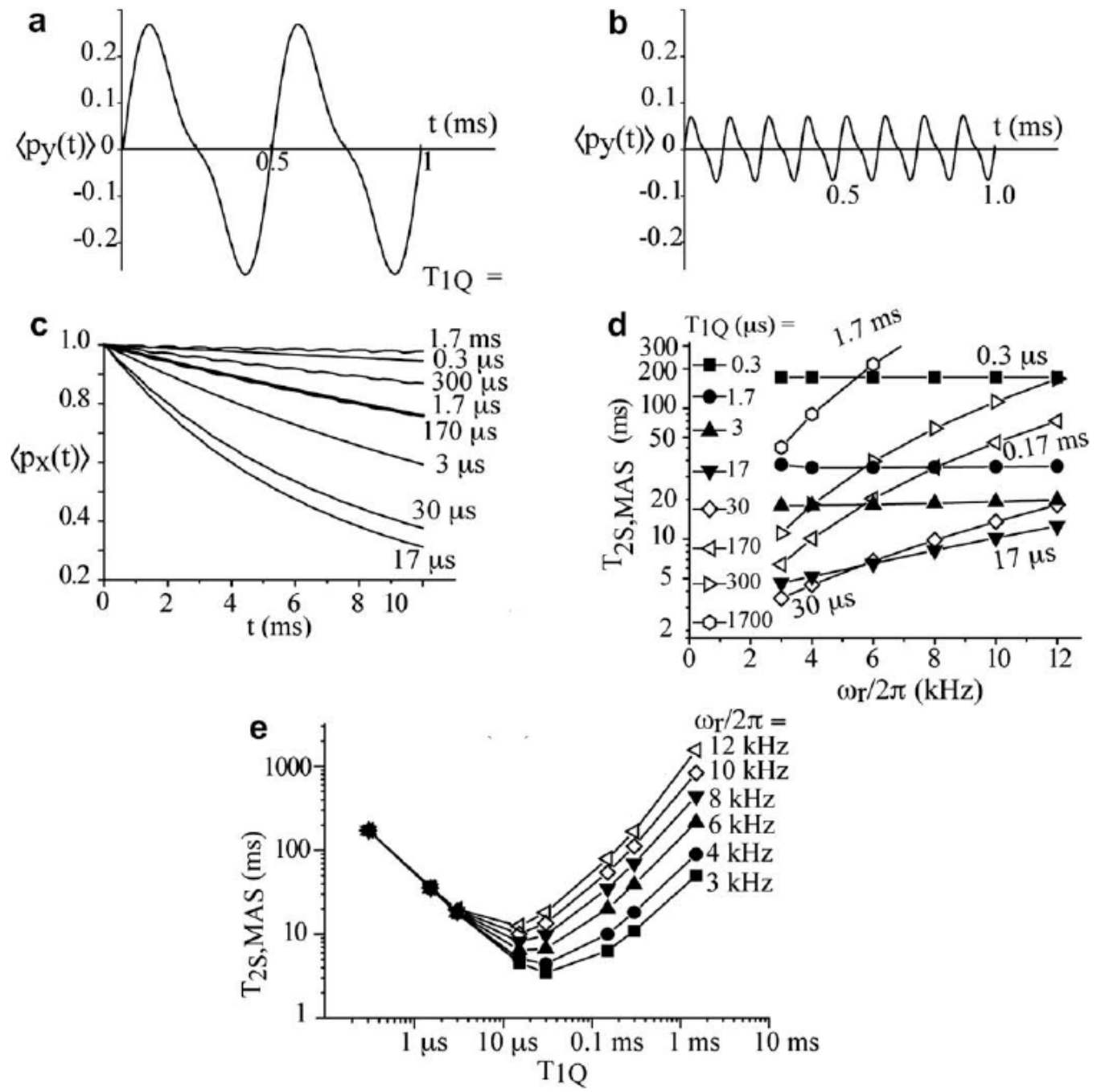

Fig. 7.9. Effects of $T_{1 Q}$ relaxation during free MAS, calculated for $L=1$. (a, b) Amplitude of the two-spin coherence of heteronuclear evolution simulated with long $\mathrm{T}_{1 \mathrm{Q}}=333 \mathrm{~ms}$ under magic angle spinning (MAS) of (a) $2 \mathrm{kHz}$ and (b) $8 \mathrm{kHz}$. (c) Simulated $\mathrm{T}_{2 \mathrm{~S}}$, MAS decay at $8 \mathrm{kHz}$ without recoupling, for various $\mathrm{T}_{1 \mathrm{Q}}$ values. (d) Spinning frequency dependence of

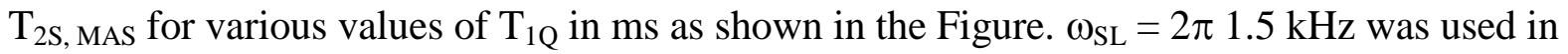
the simulations. (e) $\mathrm{T}_{1 \mathrm{Q}}$ dependence of $\mathrm{T}_{2 \mathrm{~S}}$, MAS at various spinning frequencies. 

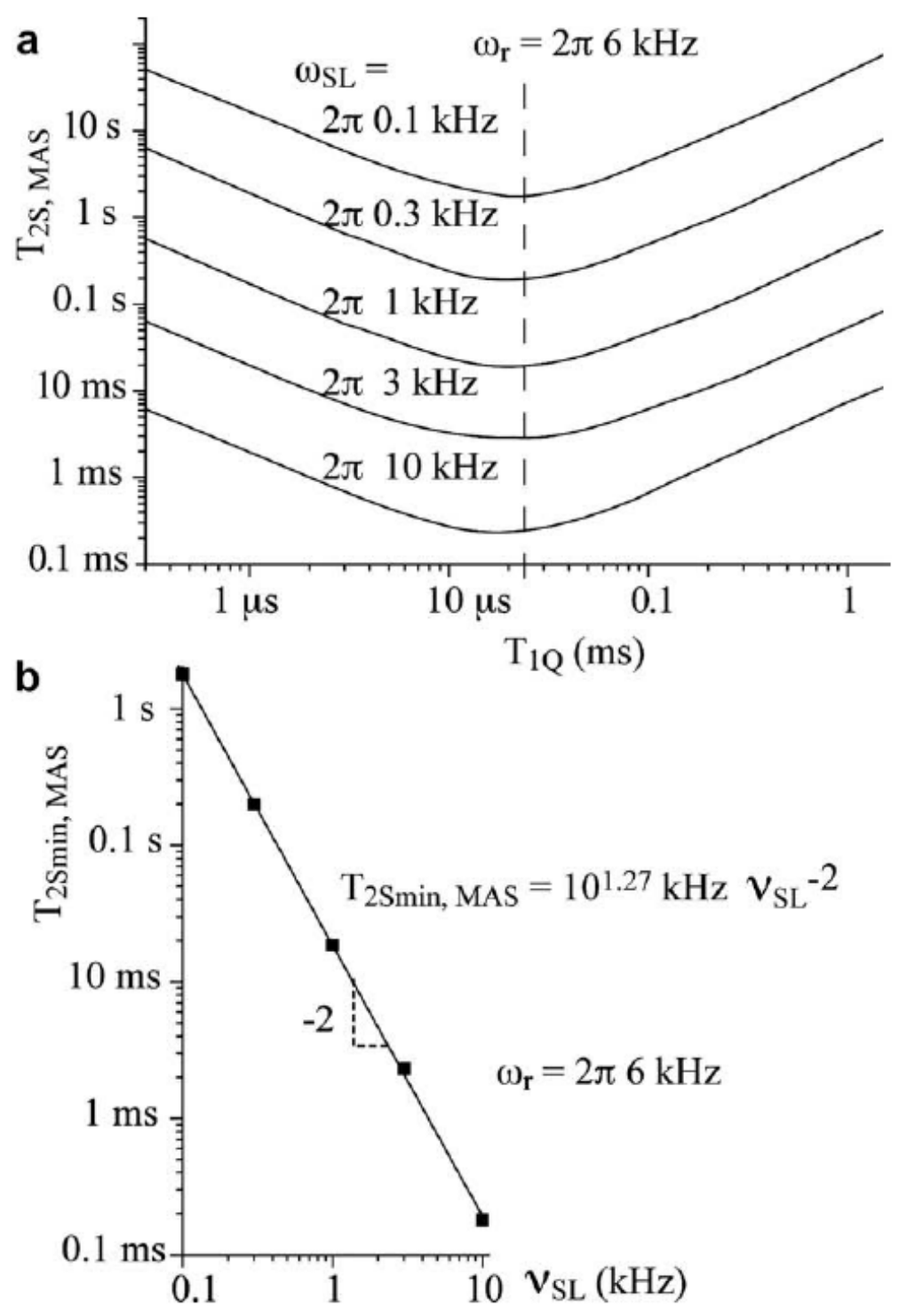

Fig. 7.10. (a) Simulated $T_{1 Q}$ dependence of $T_{2 S}$, MAS with various coupling strengths. (b) Plot

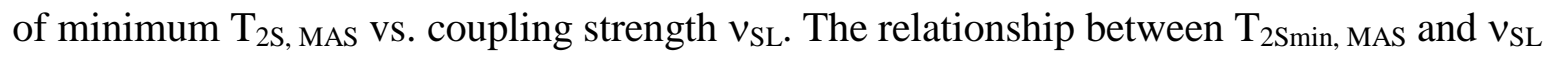
$=\omega_{\mathrm{SL}} / 2 \pi \mathrm{kHz}$ obtained from the fit is: $\mathrm{T}_{2 \mathrm{Smin}, \mathrm{MAS}}=10^{1.27} \mathrm{kHz} *\left(1 / \nu_{\mathrm{SL}}\right)^{2}$. The simulations were carried out for a spinning frequency of $\omega_{\mathrm{r}}=2 \pi 6 \mathrm{kHz}$. 


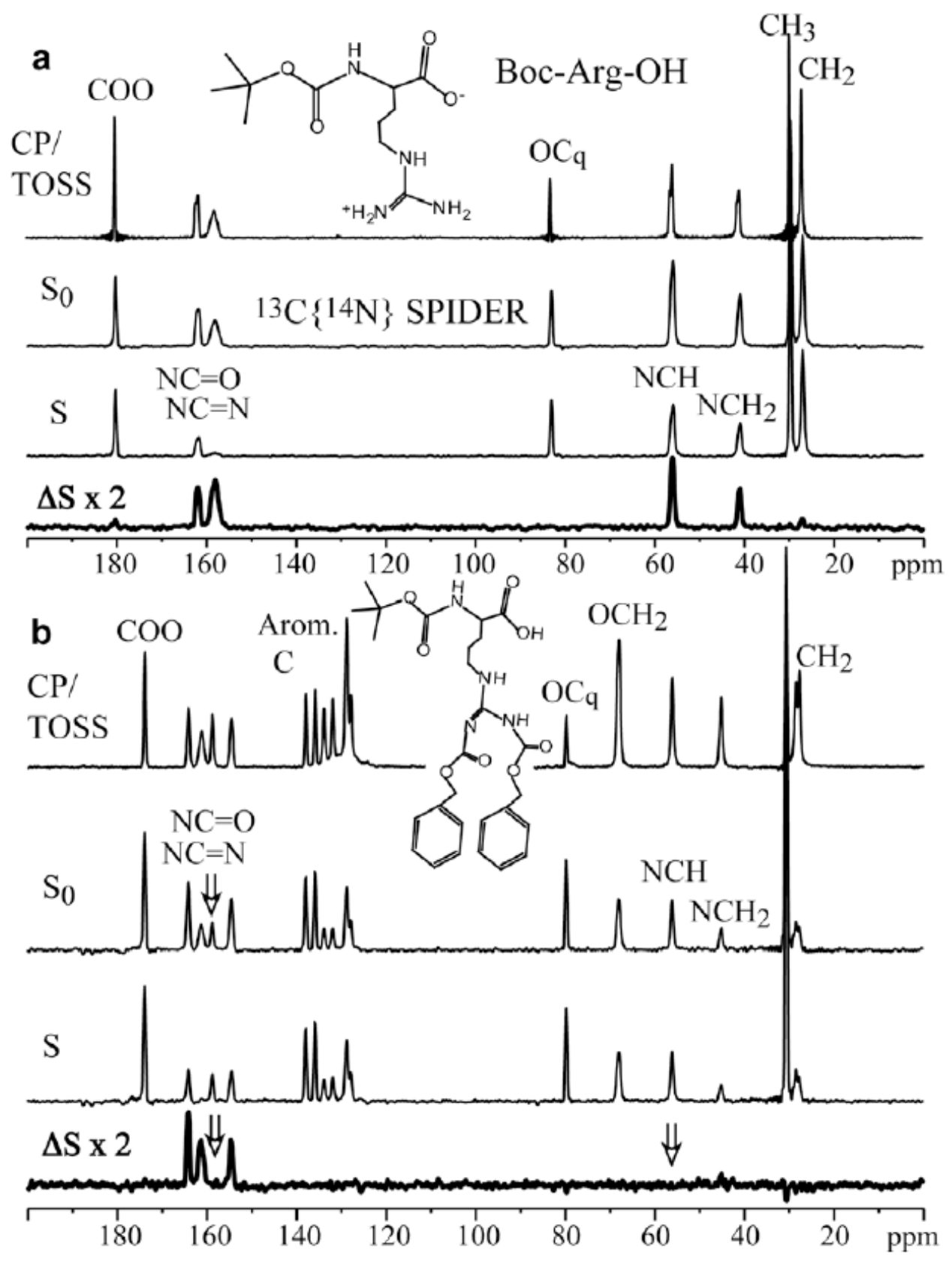

Fig. 7.11. CP/TOSS, $\mathrm{S}_{0}, \mathrm{~S}$, and $\Delta \mathrm{S}{ }^{13} \mathrm{C}\left\{{ }^{14} \mathrm{~N}\right\}$ SPIDER spectra of (a) Boc-Arg-OH, which shows the expected dephasing; (b) Boc- $\operatorname{Arg}(\mathrm{Z})_{2}-\mathrm{OH}$, which shows $\Delta \mathrm{S}=0$ and low $\mathrm{S}_{0}$ for two expected carbon signals, at 159 and 56 ppm (marked by arrows). 


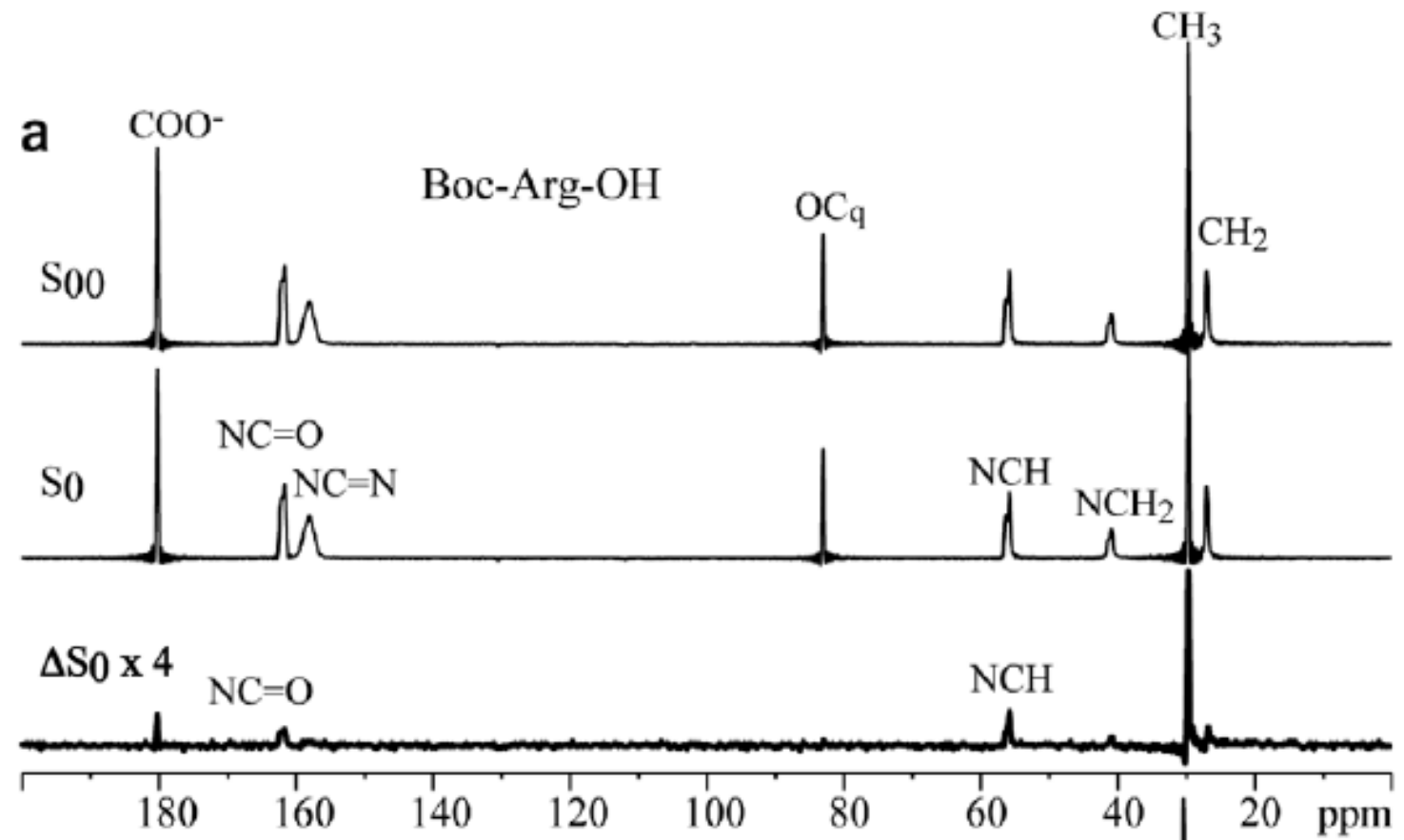

b

b $\mathrm{COOH}-\operatorname{Boc}-\operatorname{Arg}(\mathrm{Z})_{2}-\mathrm{OH}$

$\mathrm{s}_{00}$

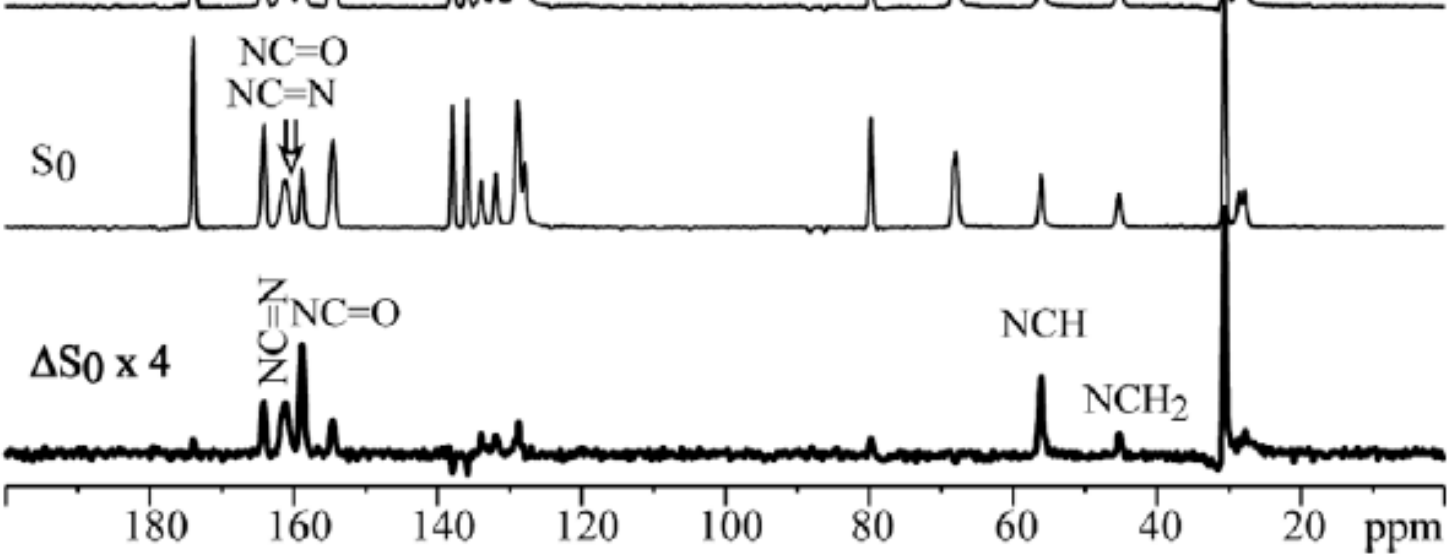

Fig. 7.12. $\mathrm{S}_{00}, \mathrm{~S}_{0}$, and $\Delta \mathrm{S}_{0}{ }^{13} \mathrm{C}$ spectra of (a) Boc-Arg-OH and (b) Boc-Arg(Z) $2-\mathrm{OH}$, which shows the largest $\Delta \mathrm{S}_{0}=\mathrm{S}_{00}-\mathrm{S}_{0}$ for the two carbon signals at 159 and $56 \mathrm{ppm}$. 




Fig. 7.13. $\mathrm{S}_{00}, \mathrm{~S}_{0}$, and $\Delta \mathrm{S}_{0}{ }^{13} \mathrm{C}$ spectra of (a) chitin and (b) (POG) $)_{10}$. Significant $\Delta \mathrm{S}_{0}$ signal is observed for all $\mathrm{C}$ bonded to $\mathrm{N}$. 


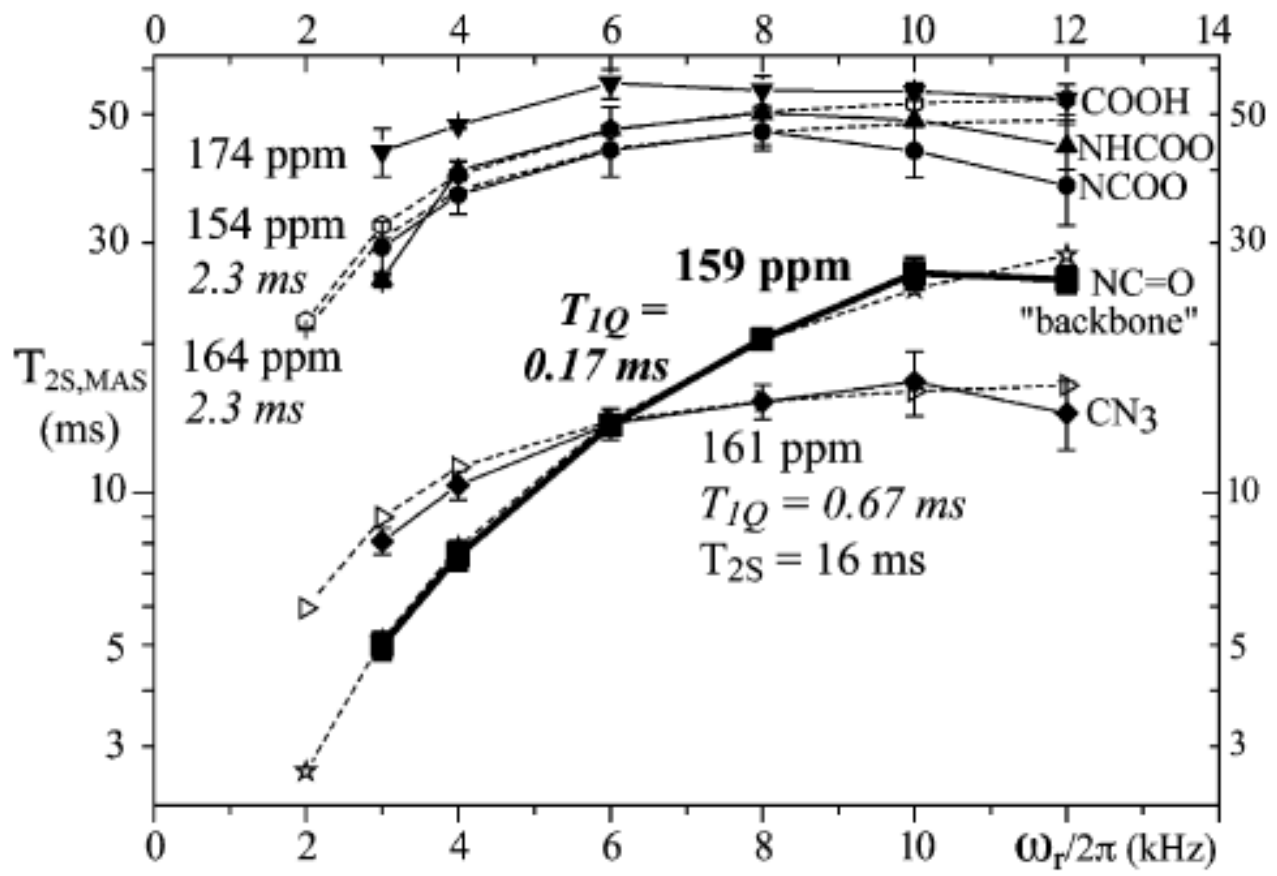

Fig. 7.14. Measurement of $T_{2 C}$, MAS as a function of spinning frequency $\omega_{\mathrm{r}}$ for various nonprotonated ${ }^{13} \mathrm{C}$ sites in Boc- $\operatorname{Arg}(\mathrm{Z})_{2}-\mathrm{OH}$. The fit curves (dashed lines and open symbols) are simulated with different values of $\mathrm{T}_{1 \mathrm{Q}}$ and $\mathrm{T}_{2}: 159 \mathrm{ppm}\left(\mathrm{T}_{1 \mathrm{Q}}=0.17 \mathrm{~ms}, \mathrm{~T}_{2}=38 \mathrm{~ms}\right), 161$ ppm (0.67 ms, $16 \mathrm{~ms}), 164$ ppm (2.3 ms, $45 \mathrm{~ms}), 154$ ppm (2.3 ms, $49 \mathrm{~ms})$. The discrepancies between the measured and fit curves at $\omega_{\mathrm{r}} / 2 \pi>10 \mathrm{kHz}$ are due to imperfect ${ }^{1} \mathrm{H}$ decoupling resulting from interference by fast spinning. The literature value of the $\mathrm{C}-\mathrm{N}$ bond length in OCNH is $0.1339 \mathrm{~nm}$, corresponding to $\delta_{\mathrm{SL}}=2 \pi 1.8 \mathrm{kHz}$, which was used in these simulations. 
a

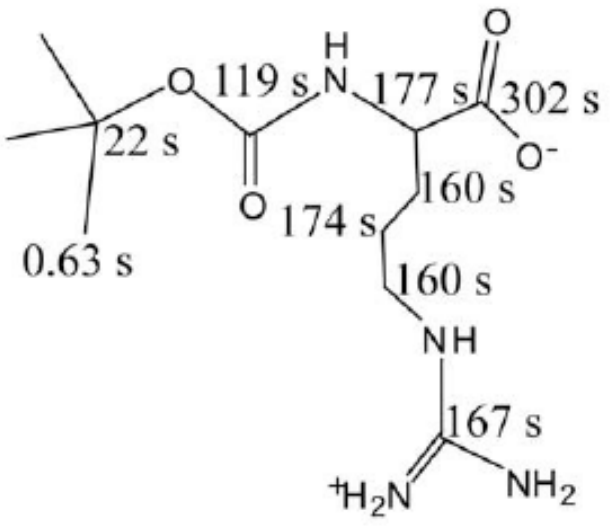

b

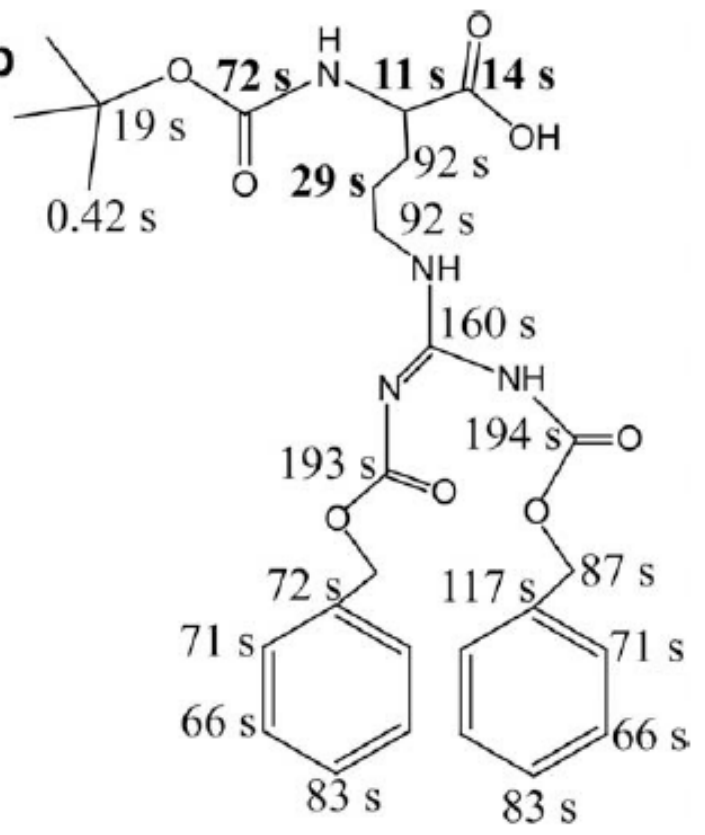

Fig. 7.15. Measured $T_{1 C}$ relaxation times in (a) Boc-Arg- $\mathrm{OH}$ and (b) Boc- $\operatorname{Arg}(\mathrm{Z})_{2}-\mathrm{OH}$. Significantly shortened $\mathrm{T}_{1 \mathrm{C}}$ values in $\operatorname{Boc}-\operatorname{Arg}(\mathrm{Z})_{2}-\mathrm{OH}$ are highlighted in bold. 

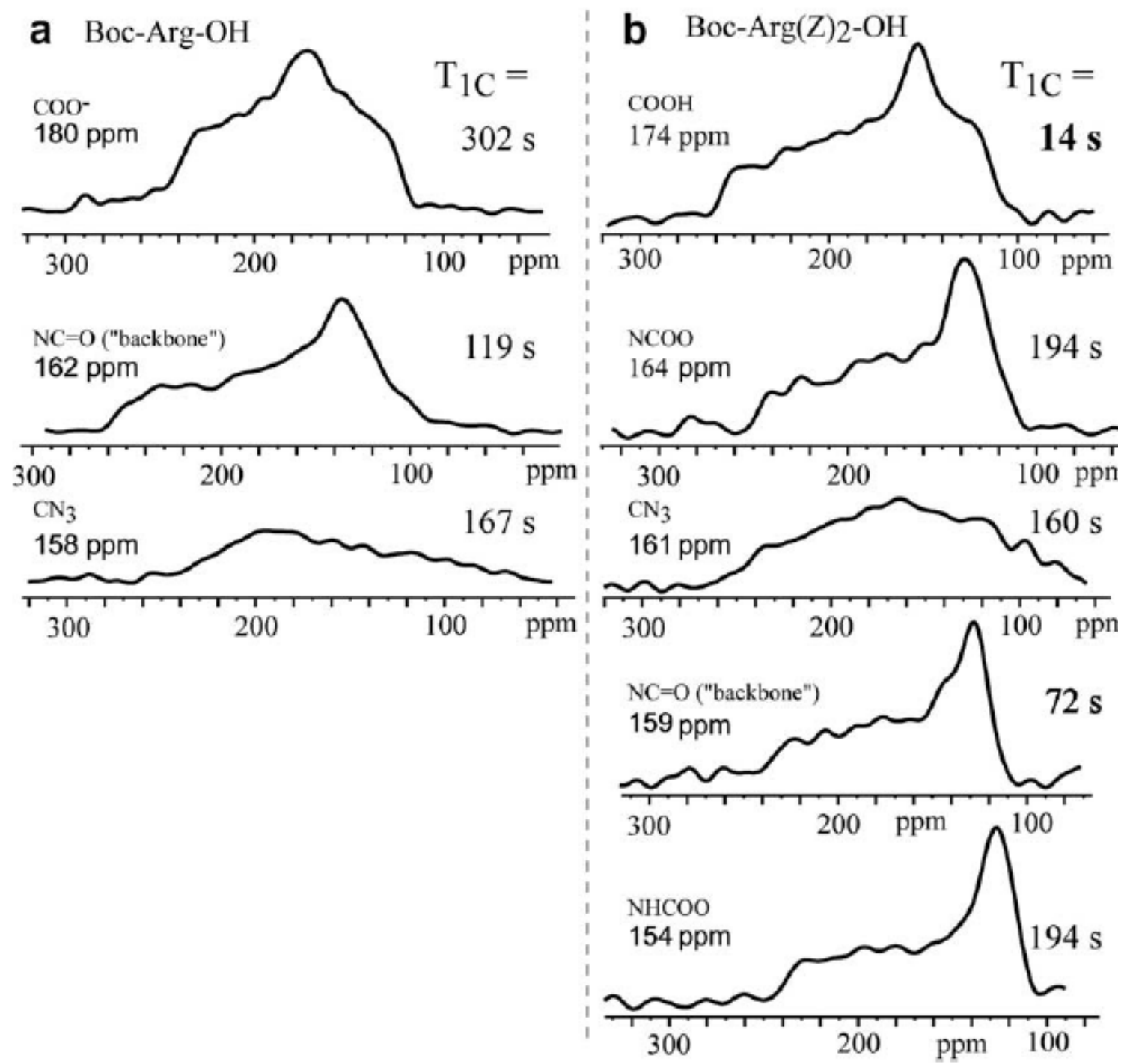

Fig. 7.16. COO powder patterns obtained as cross sections from $2 D$ SUPER spectra of (a) Boc-Arg- $\mathrm{OH}$ and (b) Boc- $\operatorname{Arg}(\mathrm{Z})_{2}-\mathrm{OH}$. The $\mathrm{T}_{1 \mathrm{C}}$ relaxation time of each site is listed to the right of its powder pattern. 


\section{Supplementary information}

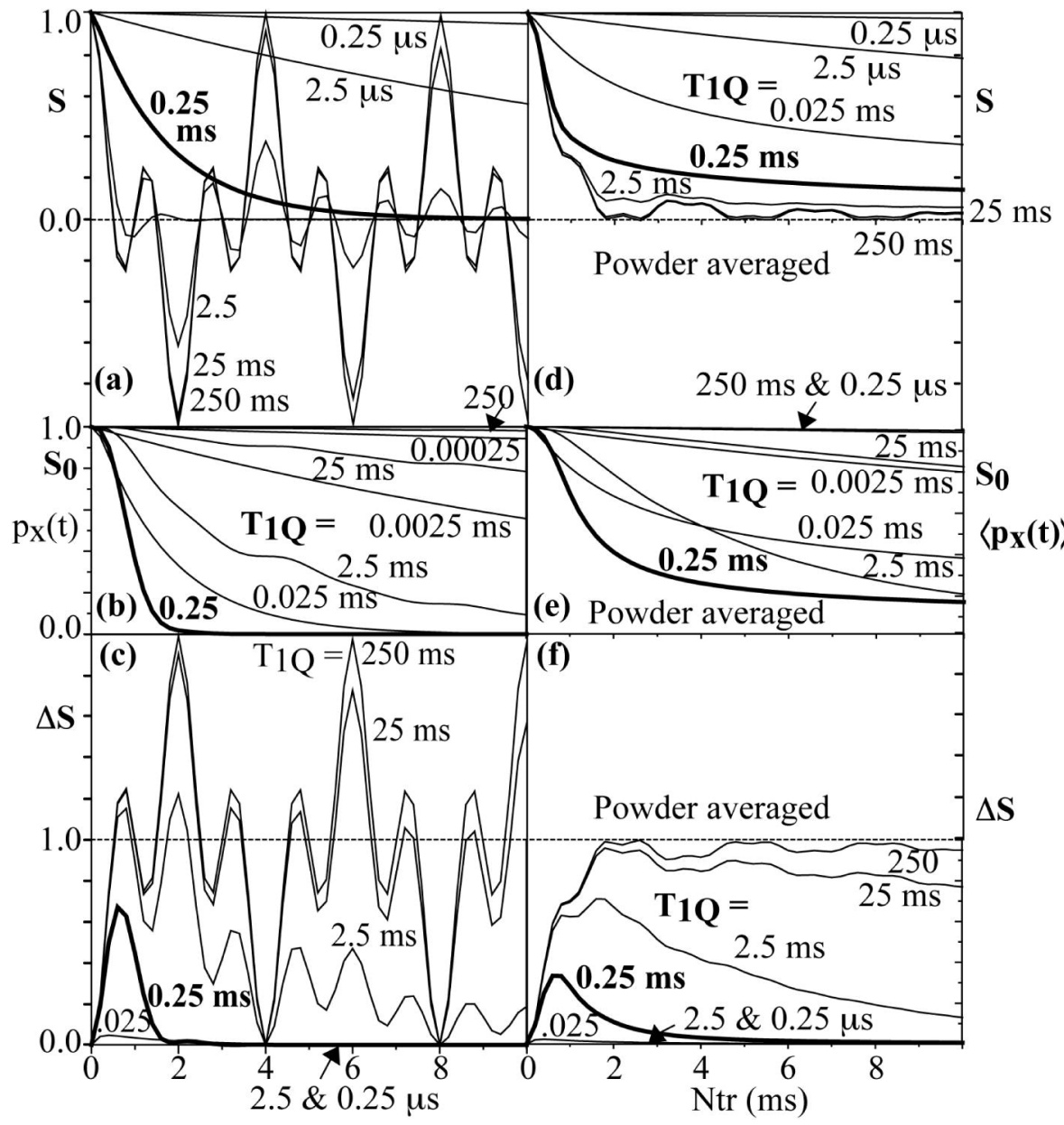

Fig. 7.S1. $\underline{S\{L\}}$ REDOR simulations for spin $\mathrm{L}=3 / 2$ with $\mathrm{T}_{1 \mathrm{Q}}$ relaxation. Single frequency

REDOR (a) $\mathrm{S}$, (b) $\mathrm{S}_{0}$, and (c) $\Delta \mathrm{S}$ simulations with $\omega_{\mathrm{SL}}=2 \pi 0.5 \mathrm{kHz}$. Powder-averaged

REDOR (d) $\mathrm{S}$, (e) $\mathrm{S}_{0}$, and (f) $\Delta \mathrm{S}$ simulations with $\delta_{\mathrm{SL}}=2 \pi 1.5 \mathrm{kHz}$. 


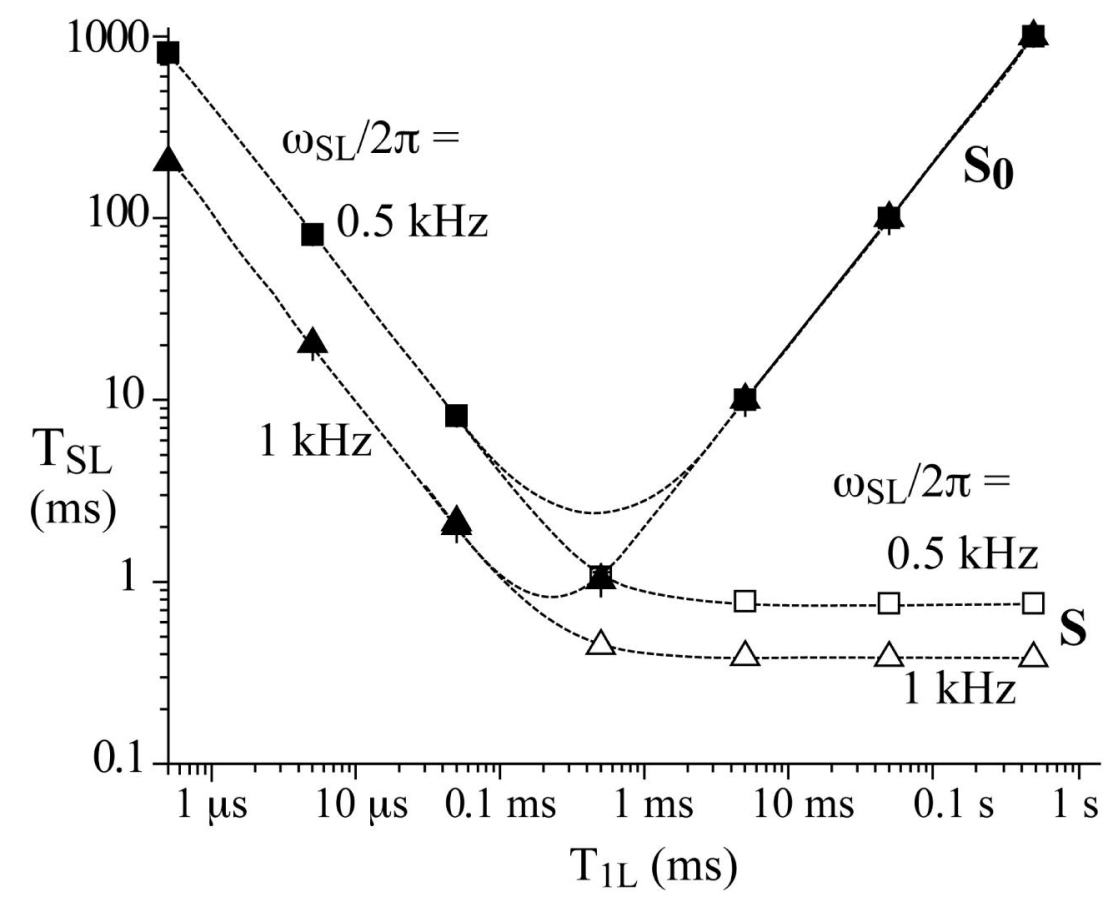

Fig. 7.S2. Decay time constants $T_{S L}$ of REDOR $S$ and $S_{0}$ for $\operatorname{spin} L=1 / 2$ as a function of $\mathrm{T}_{1 \mathrm{~L}}$, with two different coupling strengths, $\omega_{\mathrm{SL}}=2 \pi 0.5 \mathrm{kHz}$ and $\omega_{\mathrm{SL}}=2 \pi 1 \mathrm{kHz}$. 


\section{Chpater 8}

\section{General Conclusions}

\section{General discussion}

In this thesis, the application of solid-state NMR to characterize the organic-inorganic interfacial structure in bone and biomimetic polymer-calcium phosphate nanocomposites was discussed. Also, relevant solid-state NMR method improvement and development to facilitate the accurate inter-atomic distance measurements and achieve broadband highresolution NMR were documented.

The unique capability of solid-state NMR to select only the signals of organic species at the "buried" organic-inorganic interface enables the characterization of the collagen-apatite interface in bone. This study identified citrate as the major organic component at the interface, potentially the "active" biomolecule relevant to bone biomineralization. Citrate accounts for $5.5 \mathrm{wt} \%$ of total organics and covers the apatite surface at a density of 1 citrate molecule per $4 \mathrm{~nm}^{2}$ in bovine bone. With a geometrical area of $0.65 \mathrm{~nm}^{2}$, citrate occupies $1 / 6$ of the available citrate surface. The structure and geometry study of citrate revealed that citrate is about $0.4 \mathrm{~nm}$ away from the apatite surface with its backbone slightly tilted relative to the apatite surface. The three apatite-binding carboxylate groups face the apatite surface, while the non-polar $\mathrm{CH}_{2}$ groups are close to the more hydrophobic collagen matrix; this configuration "binds" the chemically very different two phases: collagen and apatite, and optimizes the mechanical properties of bone. The study also explained why apatite, rather than other calcium phosphates, forms as the main inorganic constituent in bone: the distances among the carboxylate groups of citrate match the spacing among $\mathrm{Ca}$ ions in the apatite crystal lattice. This study echoed the inquiry of the plate-like apatite crystal structure with a 3-nm thickness by proposing that citrate interferes with crystal thickening via strongly binding the apatite surface.

The proposal of citrate preventing apatite crystal thickening and thus allowing for a plate-like structure was proven by conducting biomimetic synthesis of Pluronic polymercalcium phosphate nanocomposites with various amount of citrate added. The solid-state NMR study of these nanocomposites, complemented by XRD, TEM, and SAXS, has shown that the thickness of hydroxyapatite crystals within the nanocomposites decreases with 
increasing citrate concentration. Citrate significantly modifies the interface structure of the nanocomposites, indicated by increased disordered phosphates with increasing citrate concentration, which provides a strategy for interface engineering to accomplish optimal performance of nanocomposites. Moreover, with citrate concentration comparable to that in body fluids, hydroxyapatite crystals of similar size as those in bone have been produced, which indicates that citrate is a major regulator of the apatite crystal size in bone.

As discussed in Chapter 1, the mechanical properties of bone and bone-mimic nanocomposites not only depend on the interface structure and the size of apatite crystals, but also their organic and inorganic compositions. Bone is composed of $65 \mathrm{wt} \%$ (45 vol\%) of mineral and the rest is collagen, non-collagenous proteins and water. Bone-mimic polymercalcium phosphate nanocomposites synthesized by sol-gel method often fall short on the mineral portion. The mineral content has been raised by using Lysine-Leucine diblock copolypeptide, which can form a gel at very low concentration $(0.5-4 \mathrm{wt} \%)$, to template the formation of polymer-hydroxyapatite nanocomposites. The TGA result showed that composites have been obtained with hydroxyapatite of above $50 \mathrm{wt} \%$. Solid-state NMR identified the mineral phase as hydroxyapatite and confirmed the formation of nanocomposites.

In this thesis, magic-angle spinning (MAS) was used in conjunction with all the solid-state NMR methods employed to attain high resolution NMR. However, MAS cannot spin out CSA that is comparable or larger than the spinning speed. Regular sideband suppression methods such as TOSS or PASS are not suitable for spin systems involving high$\mathrm{Z}$ spin-1/2 nuclei in a non-cubic environment with a large CSA and spectral range, due to limited excitation range of long pulses. Thus, we have adapted Gan's magic-angle turning (MAT) experiment to fast MAS, termed fast MAT, to maximize the sensitivity and accomplish broadband excitation by solving pulse timing issues. Fast MAT was demonstrated on a model compound and applied to characterize technologically important tellurides, which has showed that fast MAT can generate "infinite-speed" spectra over a spectral range up to $\sim 1.8 \gamma \mathrm{B}_{1}$ without significant distortions. The comparison between fast MAT and PASS confirmed that fast MAT is preferable when broadband excitation is required. Echo-matched Gaussian filtering has been used in offline data processing to 
improve the signal-to-noise ratio and sharing between channels after shearing to minimize spectral artifacts due to digital rounding.

REDOR NMR has been employed to select the signals of citrate out of those from the collagen matrix and quantitatively determine the distance of citrate carbons from the apatite surface. The corresponding methods for spin systems involving quadrupolar nuclei, such as SPIDER and REAPDOR, suffer from the fast relaxation of quadrupolar spins on the order of sub-millisecond; this leads to inaccurate or impossible determination of inter-atomic distance. The phenomenon was studied by analytical and numerical simulations of REDORlike spin evolution of spin-1/2 coupled to spin-1/2, 1 , or $3 / 2$. The study has showed that fast $\mathrm{T}_{1 \mathrm{Q}}$ relaxation causes reduction or vanishing of the two-spin coherence before the second recoupling period, which leads to intensity loss of the reference signal $\mathrm{S}_{0}$, in turn an overestimation of the inter-nuclear distance. This problem was demonstrated on several model compounds. Fast $\mathrm{T}_{1 \mathrm{Q}}$ relaxation also causes the shortening of $\mathrm{T}_{2}$ due to involving transient dipolar coupling evolution, which is spinning speed dependent. The minimization of $\mathrm{T}_{1 \mathrm{Q}}$ effect in SPIDER and REAPDOR was achieved with a new $\mathrm{S}_{0}$ pulse sequence with minimal recoupling.

\section{Recommendations for future research}

The role of citrate in bone and biomimetic synthesis can be further examined. First, the amount of citrate varies among different mammalian species, different bones of the same species, and the same bone of different mineral age. Thus comparative studies can be carried out to relate the citrate concentration with the apatite crystal size in vivo, the variation of citrate concentration with mineral age, and possibly abnormal citrate concentration in diseased bones. Clinical therapies using citrate control may be developed to correct anomalies of natural tissue mineralization. The basis for natural selection of citrate concentration in different bones, possibly for various requirements of mechanical properties, can be probed. Second, citrate in bone may not only affect the apatite crystal structure, but also the mineral content in the tissue, therefore, the correlation of citrate concentration with the degree of mineralization can be explored. Third, the biological origin of citrate in bone and the control of citrate concentration in body fluids can be investigated. For bone-mimic 
syntheses, the crystal size control and surface modification by citrate can be combined with polymers that can form a gel at low concentration and simulate the hierarchical structure as the collagen matrix in bone, so the structure and composition of bone can be more closely imitated. To extend the concept that small molecules such as citrate rather than highmolecular-weight proteins are indeed the main player in the biomineralization of bone, small molecules with the key functional groups of essential proteins can be used to substitute certain proteins for specific functions.

Fast MAT can be further refined by combining the advantage of simple implementation and data processing of PASS with the broadband feature of fast MAT, as described in Gan's most recent published and ongoing work. The application of fast MAT can also be expanded to other high- $\mathrm{Z}$ spins such as ${ }^{207} \mathrm{~Pb},{ }^{119} \mathrm{Sn}$, and ${ }^{195} \mathrm{Pt}$.

The effect of fast $\mathrm{T}_{1 \mathrm{Q}}$ relaxation can be included in REDOR simulation programs for more accurate determination of inter-atomic distance. The compensated $\mathrm{S}_{0}$ pulse sequence remains as a good probe for qualitatively evaluating the potential $\mathrm{T}_{1 \mathrm{Q}}$ relaxation effect. 